\title{
Writ - Claim form - Klage
}

Eine rechtsvergleichende Untersuchung zur

Klageeinleitung im englischen und deutschen Zivilprozess sowie in aktuellen Konzepten für internationale Zivilprozessordnungen

\section{Dissertation}

zur Erlangung des Doktorgrades

der Juristischen Fakultät

der Georg-August-Universität Göttingen

\author{
vorgelegt von \\ Thomas Schuster \\ aus Bukarest/Rumänien
}

Göttingen 2006 

Meinen Eltern 


\section{Danksagung}

Mein Dank gilt all den Personen, die mir bei und während der Erstellung dieser Arbeit behilflich waren und mich mit Rat, Tat oder Ablenkung unterstützt haben.

Professor Dr. Münch danke ich für die Themenanregung und die Erstellung des Erstgutachtens, Professor Dr. Spindler für die Erstellung des Zweitgutachtens. Christoph Seebo hat sich die Mühe gemacht, die Arbeit in der Rohfassung zu lesen und wertvolle Hinweise und Verbesserungsvorschläge $\mathrm{zu}$ machen. Axel Sodtalbers danke ich nicht nur für die moralische Unterstützung, sondern insbesondere auch für die wertvolle Hilfe in Textverarbeitungsfragen.

Meiner Mutter danke ich für die sprachliche Korrektur und meinem Vater für die logistische Unterstützung. Meine Eltern haben mir nicht nur die Erstellung dieser Arbeit, sondern meine gesamte Ausbildung ermöglicht.

Schließlich gilt mein besonderer Dank meiner Frau, die nicht nur inhaltlich und sprachlich zur Verbesserung der Arbeit beigetragen hat, sondern mich durch die Höhen und Tiefen begleitet hat, die mit der Erstellung der Arbeit einhergingen und ganz erheblichen Anteil daran trägt, dass ich das Projekt nicht aufgegeben habe. 
„Im Zivilprozess kommen die justizpolitischen Forderungen der Gesellschaft an den Staat meist unmittelbarer und früher zum Ausdruck als im materiellen Privatrecht.“

Franz Wieacker, Privatrechtsgeschichte der Neuzeit, S. 465 


\section{Gliederung}

Abkürzungsverzeichnis XII

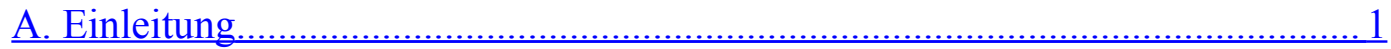

B. Die Verfahrenseinleitung im englischen Zivilprozess................................... 3

I. Das Verfahren von der angelsächsischen Zeit bis zum 19. Jahrhundert............ 3

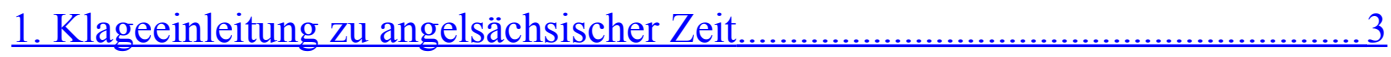

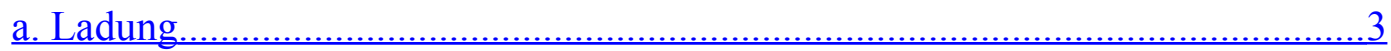

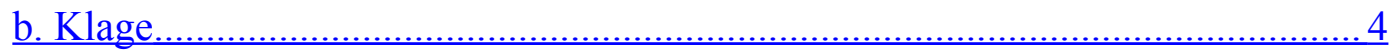

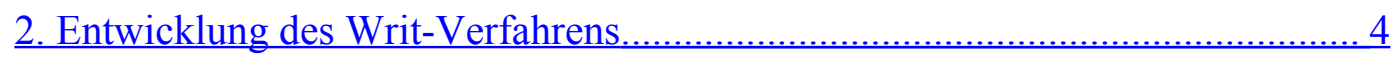

a. Writs bis zum 13. Jahrhundert..............................................................

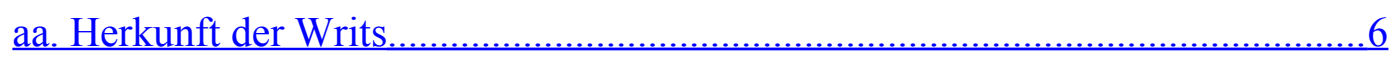

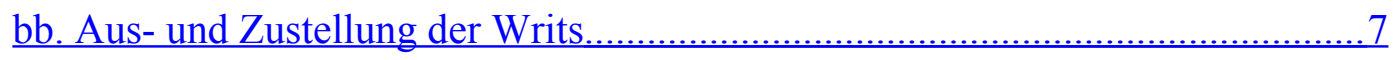

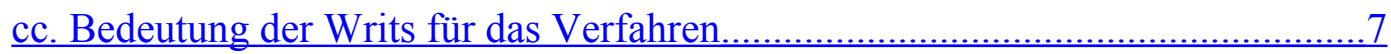

dd. Bedeutung der Writs für das materielle Recht................................................ 7

ee. Einschränkung der Befugnis der Chancery zur Herausgabe neuer Writs im

13. Jahrhundert.................................................................................... 9

b. Das Pleading-Verfahren im Common Law bis zum 17. Jahrhundert................ 10

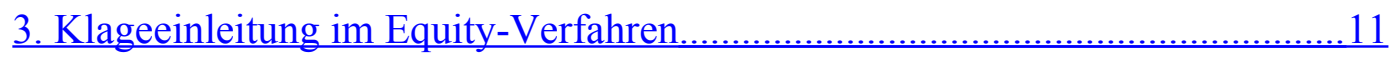

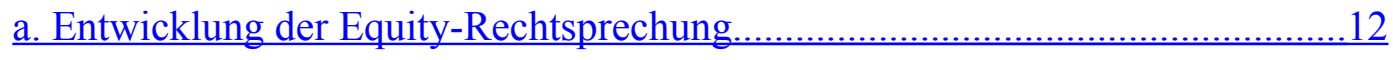

b. Das Verfahren der Equity-Rechtsprechung.......................................... 13

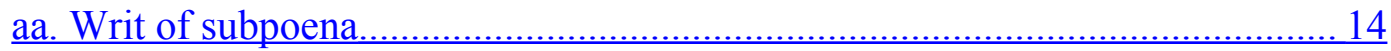

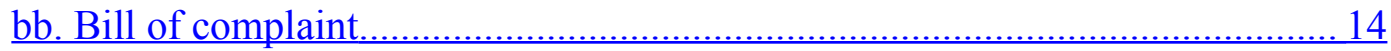




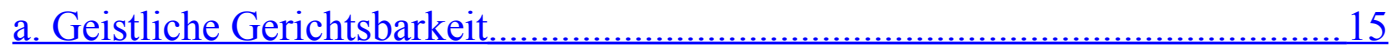

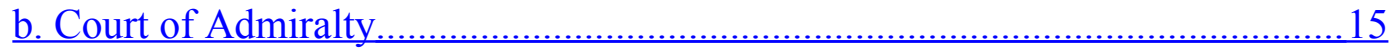

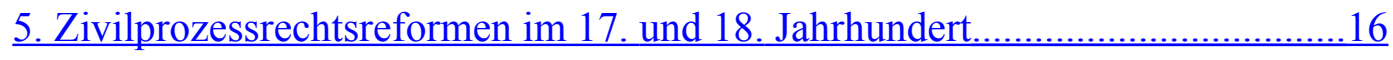

6. Römisch-kanonische Einflüsse auf das Verfahren......................................17

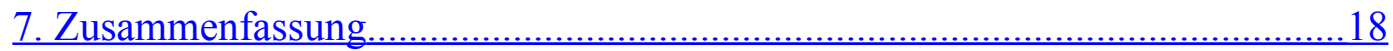

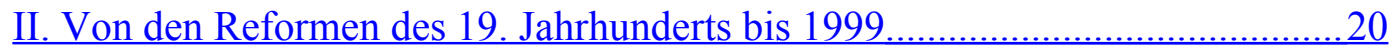

1. Die Reformen des 19. Jahrhunderts................................................... 20

a. Uniformity of Process Act 1833 und Real Property Limitation Act $1834 \ldots \ldots \ldots .21$

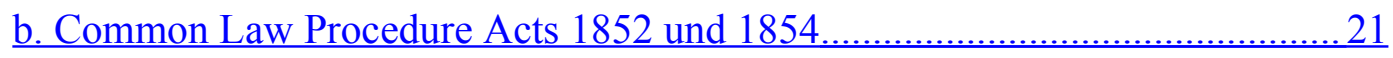

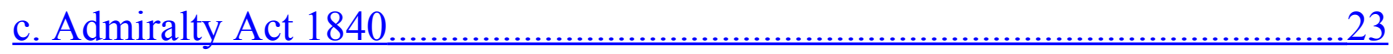

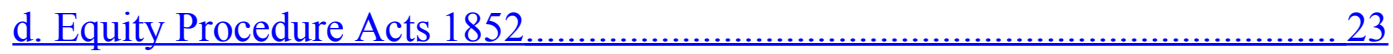

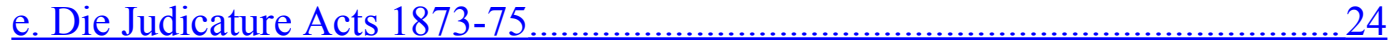

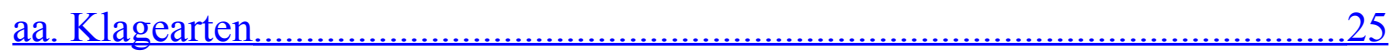

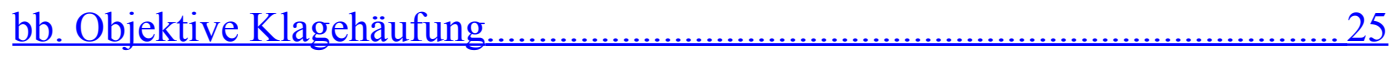

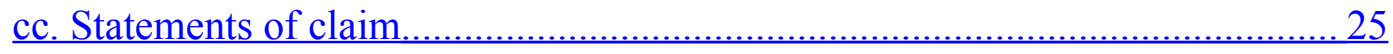

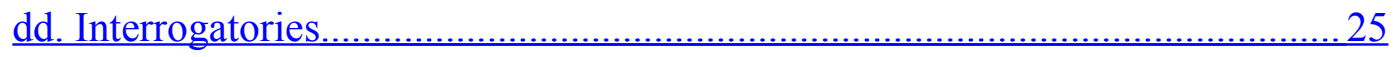

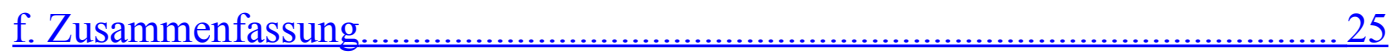

2. Der einleitende Schriftsatz im englischen Zivilprozess von den Reformen des 19. Jahrhunderts bis zur Einführung der Civil Procedure Rules 1998..................26

a. Das Verfahren vor dem High Court nach den RSC........................................ 27

aa. Ausstellung des Writ of summons (Issue of the writ of summons) ................ 27

bb. Zustellung des Writ of summons (Service of the writ of summons)..............29 
cc. Acknowledgement of Service.

dd. Statement of claim und sich daran anschließende Pleadings........................ 31

ee. Inhaltliche Anforderungen an die Pleadings.......................................... 32

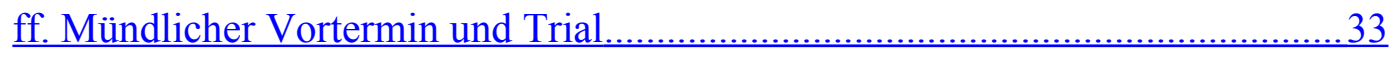

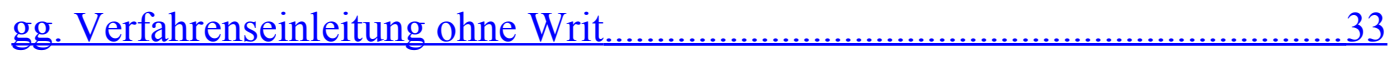

b. Das Verfahren vor den County Courts nach den CCR ................................ 34

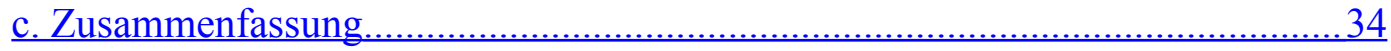

III. Der einleitende Schriftsatz nach den CPR 1998......................................... 36

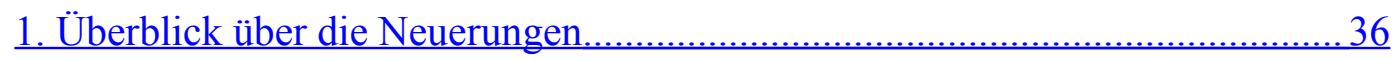

2. Die Änderungen bei der Verfahrenseinleitung ….................................... 37

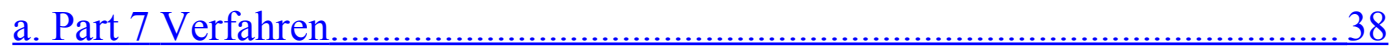

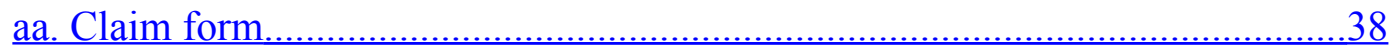

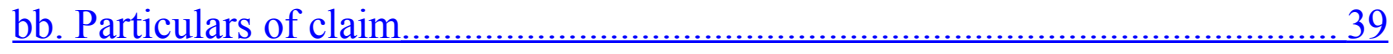

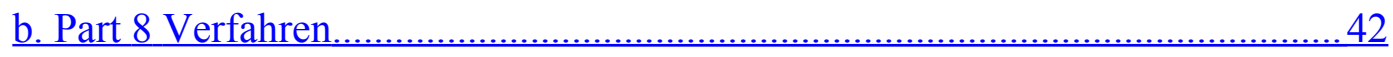

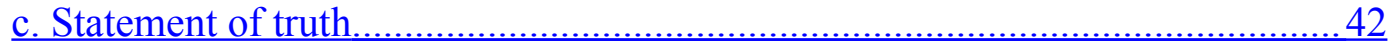

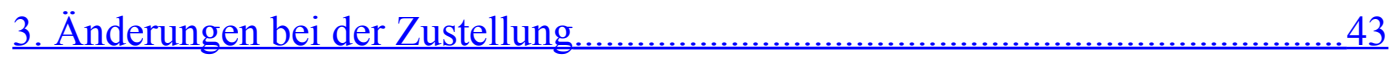

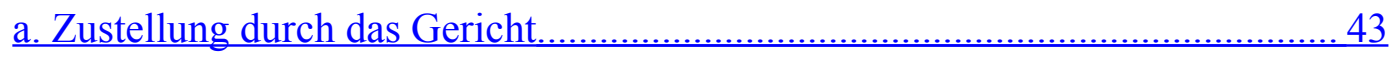

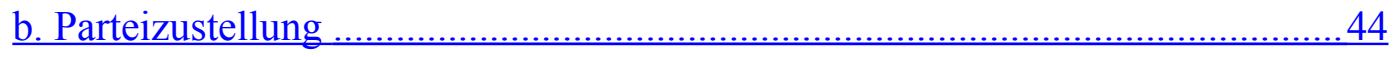

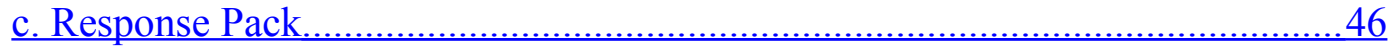

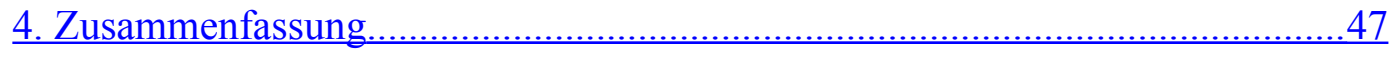

C. Rechtsvergleichende Betrachtung zum deutschen Recht.............................48

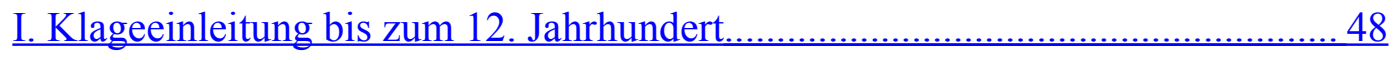




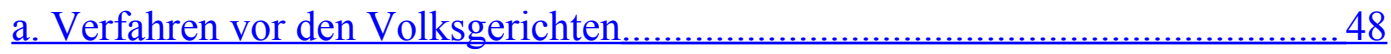

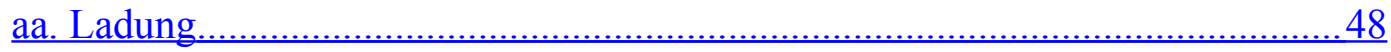

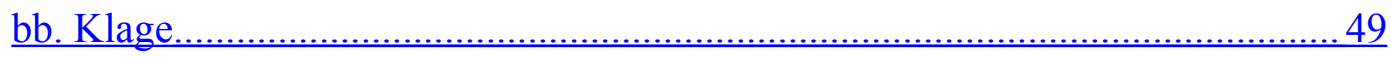

cc. Rechtsfolgen fehlerhafter Klagen ......................................................49

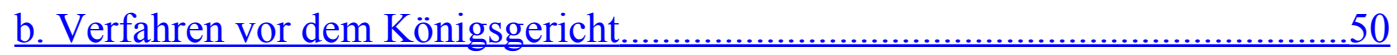

2. Klageeinleitung im Hochmittelalter...............................................................51

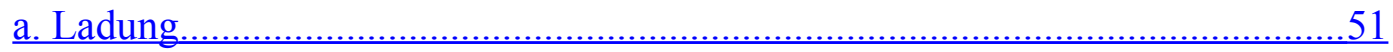

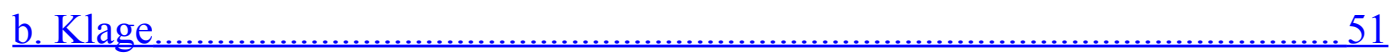

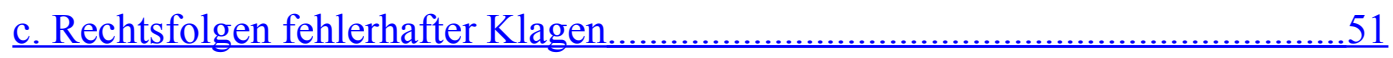

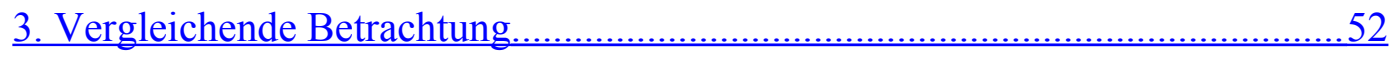

II. Das römisch-kanonische Verfahren ..................................................... 54

1. Klageeinleitung im römisch-kanonischen Verfahren................................... 54

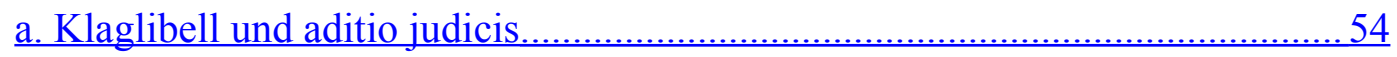

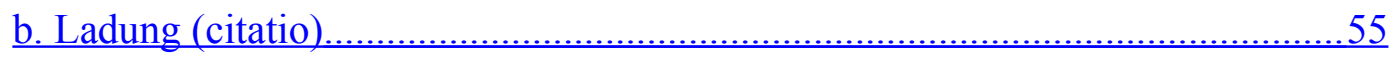

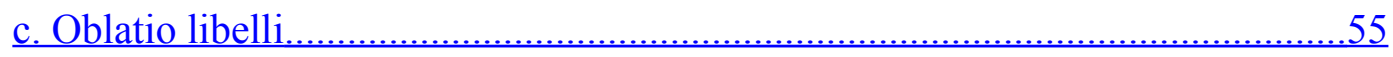

d. Weiterer Prozessverlauf....................................................................... 56

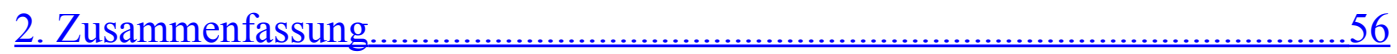

III. Die Adaption des römisch-kanonischen Verfahrens in Deutschland und die Entwicklungen des gemeinrechtlichen Prozesses........................................... 57

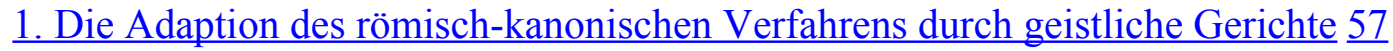

2. Die Adaption des römisch-kanonischen Verfahrens vor den weltlichen Gerichten 
a. Entwicklungen bei der Rechtsprechung des Königs....................................58

b. Entwicklungen der landesherrlichen Gerichtsbarkeit................................60

3. Der Sächsische Prozess und der gemeinrechtliche Prozess nach dem jüngsten Reichsabschied ..................................................................................61

a. Das Verfahren nach der sächsischen Gerichtsordnung von $1622 \ldots \ldots \ldots \ldots \ldots \ldots \ldots . . .61$

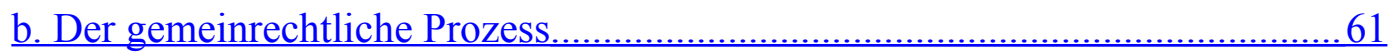

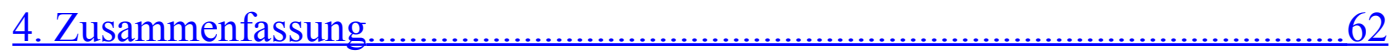

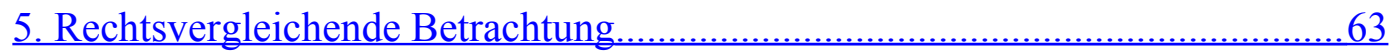

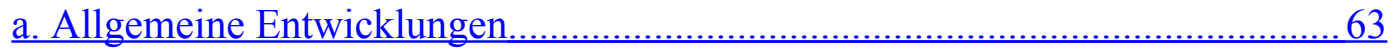

b. Entwicklungen im Common Law-Verfahren.................................................63

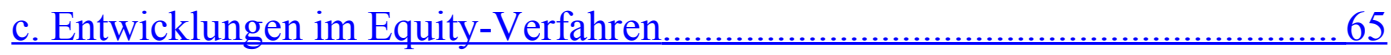

d. Gesellschaftspolitische Gründe für die zivilprozessualen Reformen..............65

IV. Die preußischen Reformversuche des 18. Jahrhunderts..........................67

$\underline{\text { 1. Verordnung über das Verfahren in Bagatellsachen von } 1739 \ldots \ldots \ldots \ldots \ldots \ldots \ldots \ldots \ldots . . \ldots 7}$

2. Project des Codicis Fridericiani Marchici 1748_........................................67

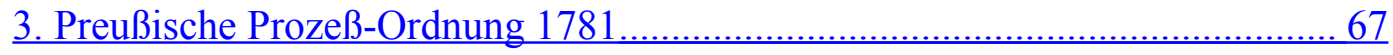

4. Allgemeine Preußische Gerichtsordnung 1793 ...................................69

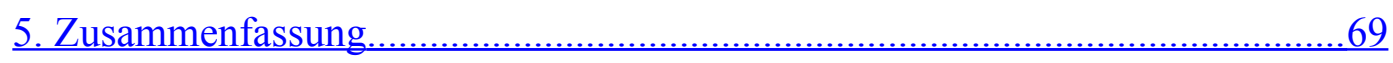

6. Rechtsvergleichende Betrachtung ….........................................................69

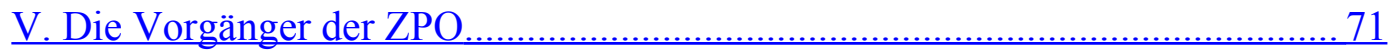

1. Der Weg zu einer einheitlichen Prozessordnung ......................................... 71

a. Die Bürgerliche Proceßordnung für das Königreich Hannover von 1850 (B.P.O). 
b. Der Entwurf einer allgemeinen Gerichtsordnung für Deutschland (Hannover-

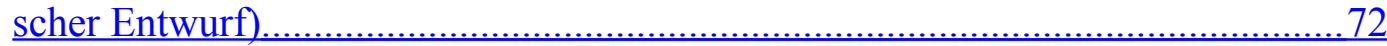

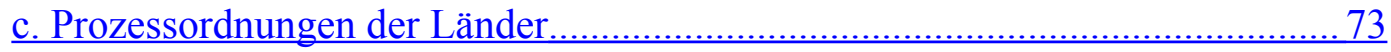

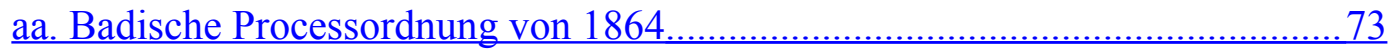

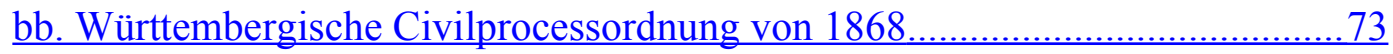

cc. Entwurf einer Process-Ordnung in bürgerlichen Rechtsstreitigkeiten für den

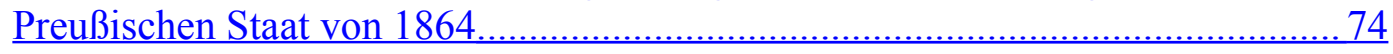

d. Der Entwurf einer Civilproceßordnung für den Norddeutschen Bund..............74

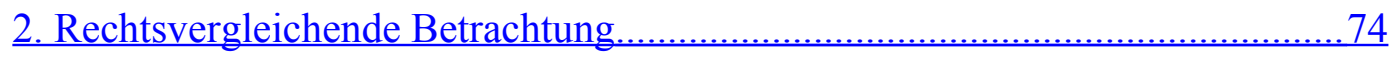

VI. Die Entwicklung der ZPO bis heute.................................................... 77

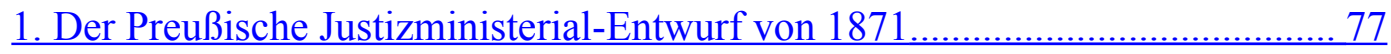

2. Die RZPO von 1877 und ihre Entwicklung zur aktuellen ZPO......................78

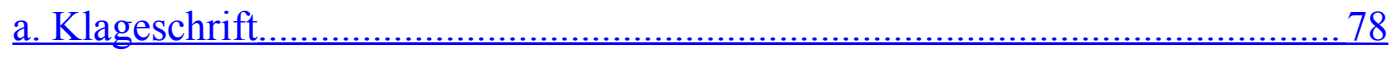

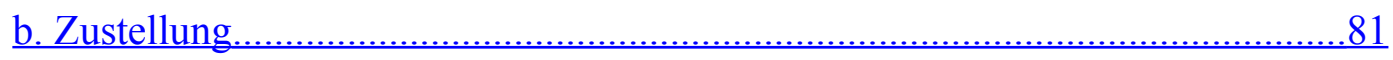

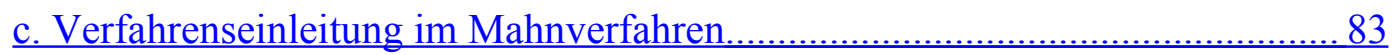

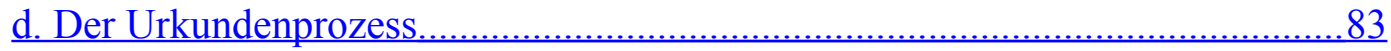

e. Weitere erhebliche Neuerungen und Änderungen..................................... 84

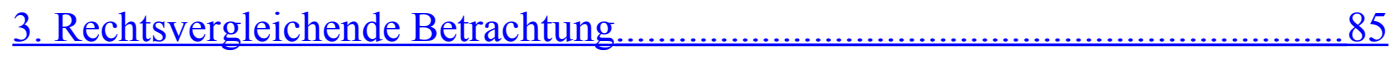

a. Inhaltliche Anforderungen an das einleitende Schriftstück......................... 85

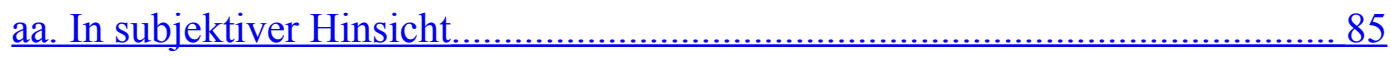

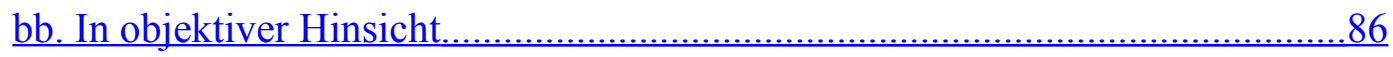

(1) Begehrter Rechtsausspruch............................................................. 86

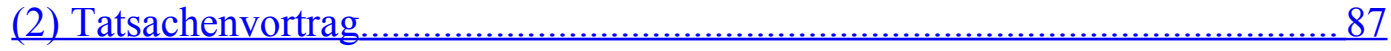


cc. In formaler Hinsicht

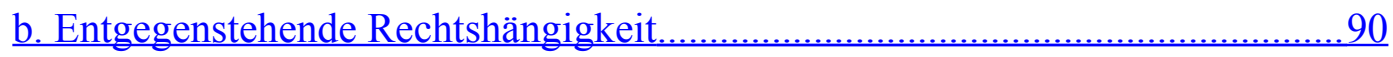

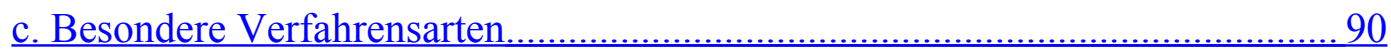

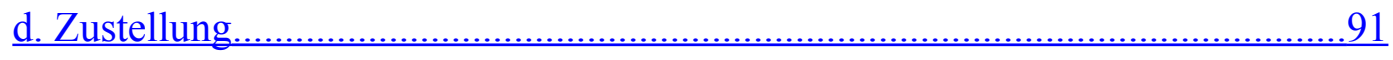

aa. Amts- oder Parteizustellung ........................................................ 91

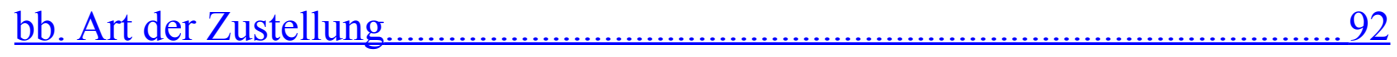

cc. Zuzustellende Dokumente................................................................. 94

e. Gesellschaftspolitische Gründe für die zivilprozessualen Veränderungen........94

D. Aktuelle Entwürfe für ein neues Zivilprozessrecht im Rechtsvergleich..........98

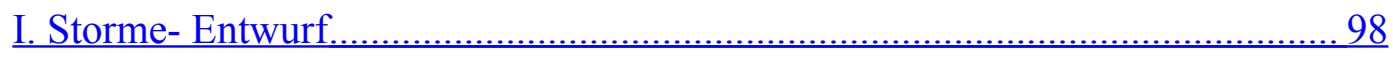

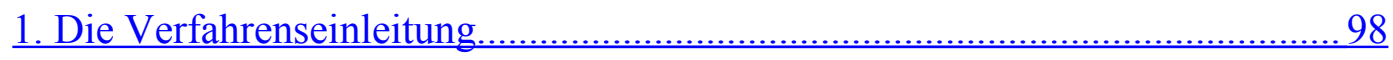

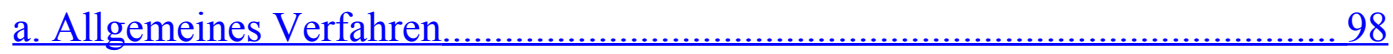

b. Inhaltliche Anforderungen an die Klageschrift............................................99

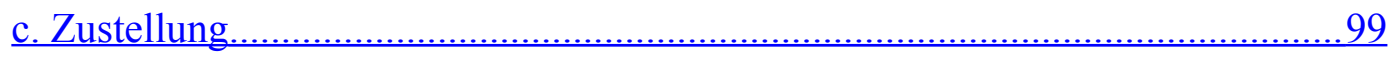

d. Verjährungsunterbrechung und lis alibi pendens.................................... 100

e. Einleitung im Mahnverfahren.......................................................... 101

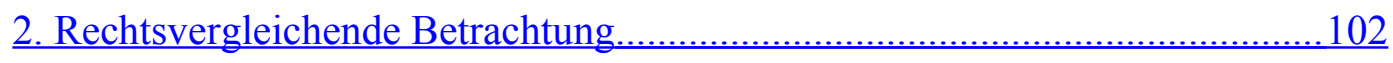

II. Principles and Rules of Transnational Civil Procedure.............................105

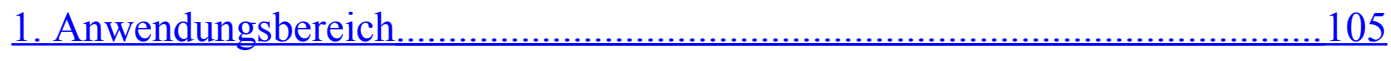

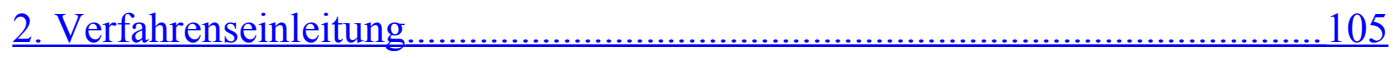

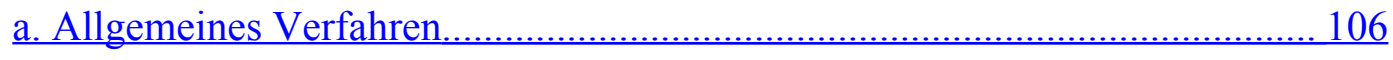

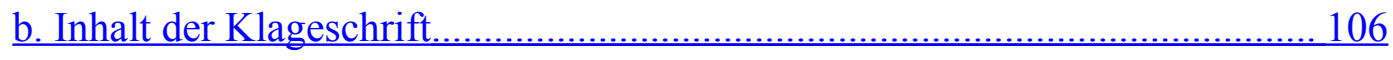


3. Rechtsvergleichende Betrachtung ...................................................... 108

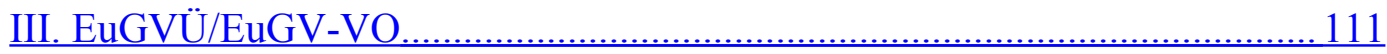

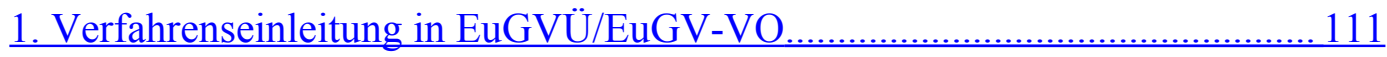

a. Das verfahrenseinleitende Schriftstück, Art. 27 Nr.2 EuGVÜ und

Art. 34 Nr. 2 EuGV-VO................................................................................. 111

b. Entgegenstehende Rechtshängigkeit...............................................112

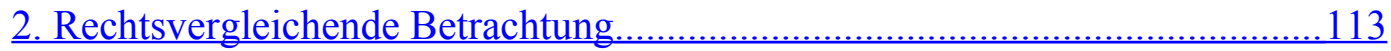

E. Abschließende Betrachtung der Entwicklungstendenzen............................115

I. System der Verfahrenseinleitung ............................................................... 115

II. Rechtshängigkeitsfolgen............................................................... 116

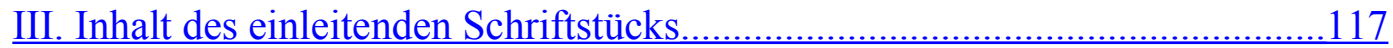

1. Anforderungen in subjektiver Hinsicht.................................................. 117

2. Anforderungen in objektiver Hinsicht..................................................... 118

3. Anforderungen in formaler Hinsicht.......................................................119

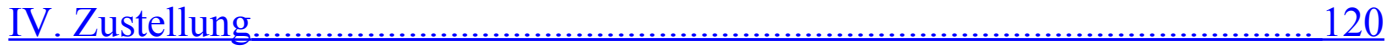

1. Amts- oder Parteizustellung?........................................................... 120

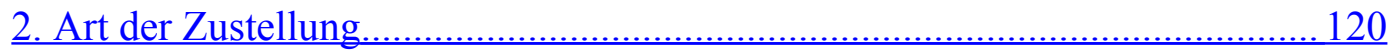

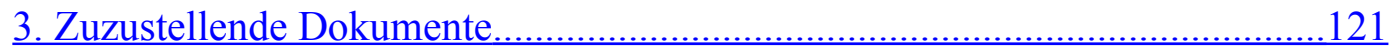

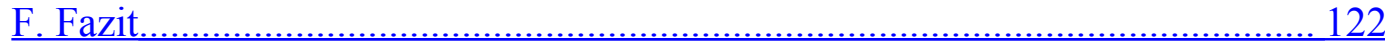

Literaturverzeichnis........................................................................ 124

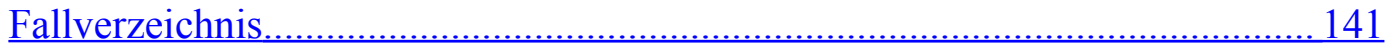




\section{Abkürzungsverzeichnis}

A.C.

A.G.O.

ALI

All E.R.

B.P.O.

nover

BGB

c.

C.A.

C.L.R

C.M.L.R.

CCR

Ch.

Ch. Rep.

Cod.I.F.M.

C.P.D.

CPR

ed.

EG

ERVVOBGH

EuGV-VO

Harvard.L.R.

H.L.

K.B.

Lex Chamav.

Lex Rib.

Lex Rom. Cur.

Lloyd's Rep.

MGH Form.

MGH Leges

no.

p.

para.

paras.

PD

PoTCP

PRoTCP

ure
Law Reports, Appeal Cases (1891 ff.)

Allgemeine Preußische Gerichtsordnung

American Law Institute

All England Reports

Bürgerliche Proceßodnung für das Königreich Han-

Bürgerliches Gesetzbuch

chapter

Court of Appeal

Cambridge Law Report

Common Market Law Review

County Court Rules

Law Reports Chancery Division (1891 ff.)

Chancery Reports

Project des Codicis Fridericiani Marchici

Law Reports, Common Pleas Division (1875-1880)

Civil Procedure Rules

edition

EG-Vertrag von Amsterdam

Verordnung über den elektronischen Rechtsverkehr beim Bundesgerichtshof

Verordnung (EG) Nr. 44/2001 des Rates vom

22.12.2000 über die gerichtliche Zuständigkeit und die Anerkennung und Vollstreckung von Entscheidungen in Zivil- und Handelssachen

Harvard Law Report

House of Lords

Law Reports, Kings Bench (1891 ff.)

Lex Frankorum Chamavorum

Lex Ribvaria

Lex Romana Raetica Curiensis

Lloyd's Law Reports

Formulae Merowingici et Karolini Aevi, Monumenta Germaniae Historica

Leges Saxonum, Leges Thuringorum, Edictum

Theoderici regis, Remedil curiensis episcopi capitula, Lex Ribvaria, Lex Frankorum Chamavorum, Lex Romana Raetica Curiensis, Monumenta Germaniae Historica, Leges

number

principle

paragraph

paragraphs

Practice Direction

Principles of Transnational Civil Prrocedure

Principles and Rules of Transnational Civil Proced- 
Q.B.

r.

rr.

RLdr.

RoTCP

RSC

S.

SS.

Teilbd.

v.

W.L.R.

ZRG Germ.

ZRG Kan.

ZRG Rom.

ZZPInt
Law Reports, Queens Bench (1891 ff.)

rule

rules

Richtsteig Landrecht

Rules of Transnational Civil Procedure

Rules of Supreme Court

section

sections

Teilband

versus

Weekly Law Reports

Zeitschrift der Savigny-Stiftung für Rechtsgeschichte, Germanistische Abteilung

Zeitschrift der Savigny-Stiftung für Rechtsgeschichte, Kanonistische Abteilung

Zeitschrift der Savigny-Stiftung für Rechtsgeschichte, Romanistische Abteilung

Zeitschrift für den Zivilprozess, Internationaler Teil

Bezüglich der weiteren Abkürzungen wird verwiesen auf:

Kirchner, Hildebert/ Butz, Cornelie

Abkürzungsverzeichnis der Rechtssprache, 5. Aufl., Berlin, New York 2003 



\section{A. Einleitung}

Der Zivilprozess ist ein Verfahren, mit dessen Hilfe Rechtsstreitigkeiten zwischen Bürgern entschieden werden sollen. Im Gegensatz zum Strafprozess, mit dem der Staat sein Regelungsmonopol durchsetzt, hat der Staat am Zivilprozess kein originäres Eigeninteresse. Daher ist die Existenz eines solchen Verfahrens nicht so selbstverständlich, wie sie uns heute erscheinen mag.

Hochentwickelte Gesellschaften, wie zum Beispiel die klassische römische Zivilisation, stellen ihren Bürgern ein System zur Verfügung, das sie nutzen können, um ihre Konflikte zu lösen. Art und Ausgestaltung dieser Systeme variieren sowohl historisch als auch geographisch beträchtlich. Dabei sind alle möglichen Systeme denkbar, angefangen mit der schlichten Nicht-Existenz eines eigenständigen staatlichen Zivilverfahrens über einen Prozess, bei dem der Staat das Spielfeld und die Regeln bestimmt, aber nur bei einem Verstoß gegen die Regeln eingreift, bis hin zu rein inquisitorischen Verfahren, die jeglicher Parteiherrschaft entzogen sind.

Die Ausgestaltung des Verfahrens spielt sich immer in einem Spannungsfeld zwischen Staat und Bürger ab: Wie weit darf der Staat regeln, schließlich geht es um den Streit zwischen Bürgern; wie weit muss der Staat regeln, um den Ansprüchen seiner Bürger Genüge zu tun. ${ }^{1}$

Unabhängig vom jeweiligen System muss sich das Verfahren an die konkreten Bedürfnisse einer Gesellschaft anpassen. Existenz und Form eines Zivilverfahrens sind demnach Indikatoren für den Entwicklungsstand und die aktuellen Bedürfnisse einer Gesellschaft. Dies gilt zwar für das Recht im Allgemeinen, aber im Zivilprozess kommen die Forderungen der Gesellschaft an den Staat meist unmittelbarer und früher zum Ausdruck, als im materiellen Recht. ${ }^{2}$

Die Rolle der Tradition in der Rechtsentwicklung ist im Verfahrensrecht noch ausgeprägter als im materiellen Recht. ${ }^{3}$ Manche meinen, dies und die Technizität des Verfahrens würden funktionale Rechtsvergleichung im Prozessrecht sinnlos machen. ${ }^{4}$ Dies mag gelten, wenn man sich auf den isolierten Vergleich einzelner Detailfragen beschränkt. Begreift man den Zivilprozess jedoch als Indikator gesellschaftlicher Entwicklungen, kann die Rechtsvergleichung, sofern sie den historischen Kontext beachtet, ${ }^{5}$ Aufschluss geben, wohin sich die eigene Gesellschaft bewegt. Diesen Zweck verfolgt die vorliegende Arbeit.

Zum Vergleich wird die englische Rechtsordnung herangezogen. Dies geschieht aus zwei Gründen: Erstens, gerade weil sich in England in den letzten 1000 Jahren eine völlig andere Rechts- und Prozesstradition herausgebildet hat. Auf der anderen Seite aber die deutsche und die englische Gesellschaft der Gegenwart einander sehr ähneln. Zweitens, weil in England mit den Civil Procedure Rules 1998 erst kürzlich eine neue und moderne Zivilprozessordnung erlassen wurde, deren Untersuchung zeigen wird, ob die Rechtstradition im Prozessrecht eine so große Rolle spielt, wie zum Teil behauptet wird. ${ }^{6}$

\footnotetext{
${ }^{1}$ Unter Bürgern werden insoweit gleichermaßen natürliche und juristische Personen verstanden.

${ }^{2}$ Wieacker, S. 465

${ }^{3}$ Vgl. dazu Kötz, JA 1991, S. 257

${ }^{4}$ Dazu Weber, ZZPInt 2000, S. 59, 63; eher negativ in der Bewertung auch Kerameus, RabelsZ 66 (2002), S. 1, 2

${ }^{5}$ Vgl. zum Verhältnis von Rechtsvergleichung und Rechtsgeschichte Zimmermann, ZEuP 7

(1999), S. 494 f.; Reimann, ZEuP 7 (1999), S. 496 ff.; Flessner, ZEuP 7 (1999), S. 513

${ }^{6}$ Vgl. Weber, a.a.O.
} 
Neben den Problemen, mit denen sich die Zivilrechtspflege auf nationaler Ebene konfrontiert sieht, ${ }^{7}$ muss sie sich den Anforderungen einer neuen, globalen Wirtschaftsordnung stellen, die verstärkt zu grenzüberschreitenden Prozessen führt. ${ }^{8}$ Dies wurde bereits in den 90er Jahren erkannt und führte zu einer verstärkten Forschungs- und Schaffenstätigkeit auf dem Gebiet des transnationalen Zivilprozessrechts. Zwei dieser neuen Konzepte, den Vorschlag der Kommission für ein europäisches Zivilprozessgesetzbuch und die „Principles and Rules of Transnational Civil Procedure", die vom ALI in Zusammenarbeit mit Unidroit erstellt werden, bezieht diese Arbeit neben dem EuGVÜ/der EuGV-VO in die vergleichende Betrachtung mit ein.

Als Betrachtungsgegenstand wurde die Verfahrenseinleitung durch den Kläger gewählt; grob umrissen also die Frage, was und wieviel der Kläger tun muss, um einen Zivilprozess in Gang zu setzen und wie das Gericht weiter mit der Sache verfährt. Auf eine genauere Begriffsbestimmung wird bewußt verzichtet, denn die Vorwegdefinition nach modernen prozessrechtlichen Kategorien kann nicht gleichermaßen den englischen oder deutschen Gegenwartsprozess und seine historischen Vorgänger erfassen. ${ }^{9}$

Die historische Betrachtung, auch wenn sie nicht im Mittelpunkt der Arbeit steht, ist jedoch zum einen unerläßlich, um insbesondere das englische Verfahren der Gegenwart zu verstehen, zum anderen, um die gesellschaftspolitischen Veränderungen des letzten Jahrtausends aufzuzeigen und wie der Zivilprozess darauf reagiert hat.

Der Betrachtungsgegenstand „Verfahrenseinleitung“ ist für die Prozessrechtsvergleichung besonders geeignet, da die Regeln über die Verfahrenseinleitung die technischen Mittel zur gerichtlichen Anwendung materiellen Rechts liefern, m.a.W. sie haben den Sinn, dem Verfahren das notwendige Instrumentarium an die Hand zu geben, seinen Zweck zu erreichen ${ }^{10}$ : Die Lösung von Konflikten unter Bürgern. Insoweit sind die Einleitungsvorschriften exemplarisch für den gesamten Zivilprozess und eignen sich in dieser Funktion für den Vergleich. Da man aber die Verfahrenseinleitung nicht völlig isoliert betrachten kann, erfolgt eine einbindende Betrachtung in das Verfahren insgesamt, sofern es erforderlich ist.

Die Untersuchung wird zeigen, dass der deutsche und der englische Zivilprozess auf ihren Wegen in die Gegenwart völlig unterschiedliche Entwicklungen durchgemacht haben. Weiterhin wird sie die Frage beantworten, inwiefern heute noch Unterschiede vorhanden sind. Schließlich wird sie beleuchten, wie die Verfahrenseinleitung in einem modernen Zivilprozess aussehen sollte und in welche Richtung sich das Zivilverfahren bewegt.

\footnotetext{
${ }^{7}$ Für England nennt Michalik, S. 116 Kosten, Verfahrensdauer und Komplexität; allgemein identifiziert Kengyel, ZVglRWiss 101 (2002), S. 260, 261 Verfahrensdauer und -kosten als Hauptprobleme.

${ }^{8}$ Kengyel, a.a.O.

${ }^{9} \mathrm{Vgl}$. insoweit auch Ranieri, Ius Commune, 28 (2001), S. 361, 363.

${ }^{10}$ Kerameus, RabelsZ 66 (2002), S. 1, 6
} 


\section{B. Die Verfahrenseinleitung im englischen Zivilprozess}

Die neue und erste echte Zivilprozessordnung Englands, die CPR 1998, bietet sich als Vergleichsmaterial an, da sie zum einen ein aktuelles und damit modernes Verfahrensrecht schafft und zum anderen das vorläufige Ende einer Rechtsentwicklung darstellt, die ca. 1000 Jahre alt ist.

Das englische Recht, insbesondere das Zivilverfahrensrecht, das mit dem materiellen Recht bis vor gut 100 Jahren untrennbar verwoben war, ist historisch einzigartig gewachsen. Daher kann man seine Entwicklung und seine Eigenheiten auch nur verstehen, wenn man seine Geschichte kennt.

\section{Das Verfahren von der angelsächsischen Zeit bis zum 19. Jahrhundert}

Infolge der Einwanderung germanischer Stämme ${ }^{11}$ auf die britische Insel im 5. und 6. Jahrhundert entwickelte sich ein germanisches Stammesrecht, welches das bis zum Rückzug der Römer ${ }^{12}$ geltende römische Recht völlig verdrängte. Stark beeinflusst wurde die Rechtsbildung in England durch den Einfall der Dänen, die zwischen dem 9. und 11. Jahrhundert große Teile Englands beherrschten und dort dänisches Gewohnheitsrecht praktizierten. ${ }^{13}$

Die angelsächsische Herrschaft und mit ihr diejenige angelsächsischen Rechts endete 1066 mit der Eroberung Englands durch die Normannen. Mit der normannischen Herrschaft entwickelte sich in England ein weltweit einzigartiges Rechtssystem, das seine aktionenrechtlichen Eigenheiten bis in die Neuzeit behauptete. Trotz diverser Reformen vollzog das englische Zivil- und Zivilprozessrecht erst im 19. Jahrhundert eine Wandlung hin zum Anspruchsdenken und weg vom Writ. 1999 bekamen England und Wales erstmalig eine einheitliche, umfassende Zivilprozessordnung.

\section{Klageeinleitung zu angelsächsischer Zeit}

Über das Recht und insbesondere die Gerichtsverfahren zu dieser Zeit ist wenig bekannt. Soweit es heute noch nachvollziehbar ist, basierte das gerichtliche Verfahren zu angelsächsischer Zeit auf germanischen Rechtsvorstellungen, wie sie zur gleichen Zeit auf dem Kontinent vorzufinden waren. Unterscheidungen zwischen Straf- und Zivilverfahren wurden nicht vorgenommen, vielmehr war Kern eines jeden Prozesses der Schuldvorwurf an den Beklagten, den dieser zu entkräften hatte. ${ }^{14}$

\section{a. Ladung}

Die Ladung des Beklagten geschah als privater Rechtsakt durch den Kläger vor Sonnenuntergang am Wohnort des Beklagten und musste diesem oder bei dessen Abwesenheit seiner Frau oder seinem Diener gegenüber ausgesprochen und durch Zeugen bewiesen werden. ${ }^{15}$

Inhaltlich musste die Ladung die zu erhebende Klage ausreichend beschreiben sowie den Gegner auffordern, an einem bestimmten Termin vor Gericht zu erscheinen. ${ }^{16}$

\footnotetext{
${ }^{11}$ Dabei handelte es sich insbesondere um Angeln, Sachsen und Juten, die auch die Bretonen verdrängten. Vgl. dazu Curzon, S. 4.

${ }^{12}$ Der Rückzug der Römer begann um 430 n.Chr.. Vgl. auch Curzon, S. 3 f.

${ }^{13}$ Holdsworth, History II, S. 15

${ }^{14}$ Holdsworth, History II, S. 103

${ }^{15}$ Holdsworth, History II, S. 103

${ }^{16}$ Bigelow, Procedure, S. 224
} 


\section{b. Klage}

Waren beide Parteien vor Gericht erschienen, musste der Kläger in einem Voreid seine gerechte Gesinnung beschwören ${ }^{17}$, bevor er sich in direkter Rede an den Beklagten wandte, um seine Klage in genau vorgeschriebenen Formulierungen vorzubringen. ${ }^{18}$

\section{Entwicklung des Writ-Verfahrens}

Mit der Eroberung Englands durch die Normannen veränderte sich auch die Rechtslandschaft in England grundlegend. Zwar wurde das vorhandene angelsächsische System nicht schlagartig abgelöst, jedoch wurde eine eigene geistliche Gerichtsbarkeit etabliert ${ }^{19}$ und mit Einführung eines strikten Feudalsystems die Jurisdiktionsgewalt an die Feudalherren übertragen. ${ }^{20}$

Die einschneidendste Veränderung geschah unter Heinrich II (1133-1189) durch die Einführung eines Königsgerichts, das widerrechtliche Landbesetzungen (disseissin) vor Ort untersuchte und die örtlichen Gerichte zunehmend verdrängte. ${ }^{21}$ Die Justizbeamten des Königs überprüften dabei die örtlichen Gewohnheiten und nahmen die für gut befundenen Regeln in das gemeine Recht (Common Law) auf. ${ }^{22}$ Durch diese Rezeption der örtlichen Regeln entstanden die Ursprünge des Rechtssystems, das heute insgesamt als Common Law bezeichnet wird.

Daneben entwickelten sich aus der Curia Regis drei weitere Gerichtshöfe mit unterschiedlichen sachlichen Zuständigkeiten. ${ }^{23}$ Der Court of Exchequer entschied in Steuerfragen. Der Court of Common Pleas war für allgemeine Zivilsachen zuständig. Der Court of King 's Bench repräsentierte die persönliche Rechtsprechung des Königs und überschnitt sich in seiner Zuständigkeit weitgehend mit dem Court of Common Pleas, war aber zusätzlich für die Überwachung der Rechtsprechung unterer Gerichte verantwortlich. ${ }^{24}$

\section{a. Writs bis zum 13. Jahrhundert}

Um Prozesse vor die königlichen Gerichte zu ziehen, bedienten sich die Könige seit Heinrich II der sogenannten Writs ${ }^{25}$ oder Brevia. Diese waren schriftliche Befehle des Königs an den Sheriff oder einen anderen Gerichtsherren, in denen die Ergreifung bestimmter Maßnahmen angeordnet wurde. ${ }^{26}$ Als solche stellten sie ein Universalinstrument zur Durchsetzung des königlichen Willens dar.

Für das Gerichtsverfahren bedeutete dies, dass sich ein Kläger von der Chancery, der königlichen Kanzlei, gegen Gebühr ein Writ ausstellen lassen musste, das dem zuständigen Gerichtsherren die Entscheidung des Streits auferlegte. ${ }^{27}$ Dieses Schriftstück wurde als Original Writ bezeichnet und diente ausschließlich der Verfahrenseinleitung. ${ }^{28}$

\footnotetext{
${ }^{17}$ Pollock/Maitland, History of English Law I, S. 40; arglistige Klagen waren mit Strafe bedroht. Vgl. Holdsworth, History II, S. 105.

${ }^{18}$ Holdsworth, History II, S. 105

${ }^{19}$ Baker, S. 14; Curzon, S. 17

${ }^{20}$ Curzon, S. 17, 148

${ }^{21}$ Baker, S. 15; Caenegem, History, S. 24

${ }^{22}$ Baker, S. 25

${ }^{23}$ Caenegem, History, S. 25

${ }^{24}$ Pollock/Maitland, I, S. 519 ff., 572 f.

${ }^{25}$ Nach wohl gängigster Ansicht ist Writ die altenglische Form des Partizip II von to write und bedeutet mithin geschrieben.

${ }^{26}$ Hollond, C.L.J. 8 (1942/44) S. 15 ff.; Peter, S. 19

${ }^{27}$ Peter, S. 19
} 
Das mit dem königlichen Siegel versehene Writ $^{29}$ enthielt den Namen und die Adresse der Parteien, eine kurze Beschreibung des Tatbestandes aus Klägersicht und den Befehl des Königs, der sich nach Sachverhaltslage richtete und auf Vorladung des Beklagten, Rückgabe von Sachen an den Kläger, Aufgebot von Geschworenen usw. lauten konnte. ${ }^{30}$

Rex vicecomiti salutem. Questus est mihi N. quod R. iniuste et sine iudicio desseisivit eum de communi pastura sua in illa villa que pertinet ad liberum tenementum suum in eadem villa, vel in illa alia villa, post ultimam transfretationem meam in Normanniam. Et ideo tibi precipio quod si predictus $\mathrm{N}$. fecerit te securum de clamore suo prosequendo, tunc facias duodecim liberos et legales homines de visneto videre pasturam illam et tenementum, et nomina eorum inbreviari. Et sumone eos per bonos summonitores quod tunc sit coram me vel iusticiis meis parati inde facere recognitionem. Et pone per vadium et salvos plegios predictum R., vel bailivum suum si ipse inventus non fuerit, quod tunc sit ibi auditurus illam recognitionem. Et habeas ibi summonitores et hoc breve et nomina plegiorum. Teste etc.

Der König mit Grüßen an den Sheriff. Der N. hat sich bei mir beschwert, dass der R. ihm unrechtmäßig und ohne Gerichtsurteil im Zeitraum seit meiner letzten Überfahrt in die Normandie die Nutzung seiner Weidegerechtigkeit in jenem Landgut verwehrt hat, die zu seinem freien Grundstück in demselben oder einem anderen Landgut gehört. Und daher befehle ich Dir, wenn genannter N. Dir Sicherheit für seinen Anspruch stellt, dafür zu sorgen, dass das Weideland und das Grundstück (des N) von zwölf freien und rechtschaffenen Männern aus der Nachbarschaft begutachtet werden und dass ihre Namen auf dieser Urkunde festgehalten werden. Und lass' sie durch gute Gerichtsboten laden, dann vor mir oder meinen Richtern zu erscheinen, bereit, ihr Urteil abzugeben. Und lade oben genannten R., oder seinen Verwalter, wenn er selbst nicht aufzufinden ist, unter der Leistung von Grundsicherheiten und verlässlichen Pfändern, dass er dann dort ist, um das Urteil zu hören. Und die Gerichtsboten, dieses writ und die Bezeichnungen der Pfänder müssen auch da sein. Zeuge (für richtige Ausfertigung), etc. ${ }^{31}$

Da sich die Sachverhalte ständig wiederholten, begannen die Schreiber der Chancery auf frühere Formulierungen zurückzugreifen. Dadurch entstanden Formulare für die häufigen Fälle der nach Common Law zu ahndenden Rechtsverletzungen. In der ersten bekannten Zusammenstellung im späten 12. Jahrhundert bei Glanvill $^{32}$ erscheinen über 70 fest vorformulierte Writs, in deren Text lediglich Namen und Adressen der Parteien einzutragen waren. ${ }^{33}$ Im Laufe der Zeit erhielten die Writs eigene Namen, die sie charakterisierten. So gab es das Writ of novel disseis-

\footnotetext{
${ }^{28}$ Davon zu trennen sind die sog. Juridical Writs, die als verfahrensleitende Maßnahmen vom Gericht erlassen wurden. Vgl. Blackstone, Commentaries III, S. 282; Pollock/Maitland, I, S. 19

${ }^{29}$ Die Original writs wurden bis ca. 1730 in lateinischer Sprache verfaßt. Blackstone, Commentaries III, S. 322

${ }^{30}$ Peter, S. 19 f.

${ }^{31}$ Glanvill XIII, 37, zitiert bei Hall S. 169 Ab „Et sumone“ etc. ergänzt nach Glanvill XIII, 33, Hall S. 167.

${ }^{32}$ Das Tractatus de legibus et consuetudinibus regni Angliae gilt als das älteste Rechtsbuch des Common Law. Vgl. Holdsworth, History II, S. 188 ff.; Pollock/Maitland, I, S. 162 f.

${ }^{33}$ Peter, S. 20
} 
in, Writ of right, Writ of detinue, Writ of mort d'ancestor, Writ of debt, Writ of trespass usw. ${ }^{34}$

Die Writs enthielten einen Gruß an den Lehnsherren oder den Sheriff, an den sie gerichtet waren. Darauf folgte der Befehl des Königs, der je nach Writ variierte. ${ }^{35}$ Im Laufe der Zeit wurden immer mehr verschiedene Writs verwendet. ${ }^{36}$ Erklärt wird dies damit, dass sich die Justizverhältnisse änderten und die Writs als obligatorische Einleitungsinstrumente diesen Veränderungen angepasst werden mussten. ${ }^{37} \mathrm{Im}$ gleichen Maße, in dem sich der Zuständigkeitsbereich der Common Law Gerichte ausdehnte, mussten neue Writs für die Tatbestände geschaffen werden, für die die Krone die Zuständigkeit der Common Law Gerichte begründen wollte. ${ }^{38}$ Da der Grundsatz ,no writ, no right" galt, war die Schaffung neuer Writs unerlässlich, um Klägern den Zugang zu Gericht zu ermöglichen. Dazu wurden neue Formulare geschaffen und gebräuchliche verändert oder ergänzt. ${ }^{39}$ Anfänglich war die Zahl der Writs so klein, dass sie in einem Korb Platz fanden. ${ }^{40}$ Mit steigender Zahl ergab sich aber das Bedürfnis, die Writs zu ordnen. Gleichzeitig oder nur unerheblich später begannen die Schreiber der Chancery damit, die gebräuchlichsten Writs in Sammlungen zusammenzustellen. Diese Sammlungen, deren älteste überlieferte aus dem Jahr 1227 stammt, wurden als Register of (original) Writs bezeichnet. ${ }^{41}$ Sie dokumentieren anschaulich die starke Vermehrung der Writs. ${ }^{42}$

\section{aa. Herkunft der Writs}

Geschriebene königliche Befehle, die den späteren Writs ähneln, existierten schon zu angelsächsischer Zeit in angelsächsischer Sprache. ${ }^{43}$ Auch in der Folgezeit unter normannischer Herrschaft finden sich derartige Befehle, die allerdings keinen fixierten Wortlaut aufwiesen. ${ }^{44}$ Die feste Form erhielten die Writs als verfahrenseinleitende Schriftstücke erst unter Heinrich $I I .{ }^{45}$ Dabei wurden Formulare des normannischen Rekognitionsverfahrens übernommen, ${ }^{46}$ welche wiederum fränkischer Herkunft sind.$^{47}$ Es gibt aber auch ältere Vorbilder aus römischer Zeit. Insbesondere das Writ praecipe quod reddat lässt sich sogar auf eine Vorlage des ostgotischen Ministers, Staatsmannes und Gelehrten Cassiodor (ca. 490-583) zurückführen. ${ }^{48}$ Die ähnliche Struktur der Urkunden legt den Schluss nahe, dass die ostgotischen Formulare ohne den Umweg über Franken und Normannen direkt

\footnotetext{
${ }^{34}$ Umfassend dazu die Übersicht bei Caenegem, History, S. 22.

${ }^{35}$ Weitere ausführliche Beispiele mit Übersetzungen finden sich bei Peter, S. $21 \mathrm{ff}$..

${ }^{36}$ Peter, S. 20

${ }^{37}$ Peter, S. 44

${ }^{38}$ Peter, S. 44

${ }^{39}$ Bieresborn, S. 411

${ }^{40}$ Blackstone, III, S. 49

${ }^{41}$ Maitland, Forms of Action, S. 41

${ }^{42}$ Während Sammlungen von 1227 bzw. 1230 noch 56 bzw. 58 Writs enthielten, (Maitland, Collected Papers II, S. 130 ff; 135 ff.) waren es in einer 20 Jahre jüngeren Sammlung schon 121 Writs, (a.a.O. S. 142 ff.) eine Generation später waren es über 170 (a.a.O. S. 156 ff.) und um 1270 bereits 322. In einer Sammlung des frühen 14. Jahrhunderts finden sich bereits 471 Writs, (a.a.O.) von denen allerdings nicht alle Bedeutung für den Prozess hatten. (Peter, S. 46)

${ }^{43}$ Eine Sammlung solcher Urkunden aus der Zeit von 970 bis 1070 findet sich bei Harmer.

${ }^{44}$ Bigelow, S. 147

${ }^{45}$ Peter, S. 65

${ }^{46}$ Brunner, Entstehung der Schwurgerichte, S. 309 ff. und $404 \mathrm{ff}$.

${ }^{47}$ Schulz, 54 (1942) Juridical Review, S. 1 ff.

${ }^{48}$ Schulz, 54 (1942) Juridical Review, S. 1 ff.
} 
nach England gelangten, um dort als Vorlage für das Writ praecipe quod reddat zu dienen. ${ }^{49}$

\section{bb. Aus- und Zustellung der Writs}

Die Writs wurden ohne Anhörung des Beklagten auf Antrag des Klägers in der Chancery ausgestellt. Welches Writ beantragt wurde, lag in den Händen des Klägers. Ihm oblag es, das richtige Writ für seinen Fall auszuwählen. Anfänglich, als es nur wenige Writs gab, war die Auswahl relativ einfach, insbesondere, da die Schreiber der Kanzlei dem Kläger bei der Auswahl behilflich waren ${ }^{50}$. Je mehr Writs es gab, desto schwieriger wurde dieses Unterfangen, so dass ab dem späten 13. Jahrhundert Anwälte den Klägern bei der Auswahl der Writs gegen Gebühr zur Seite standen. ${ }^{51}$

Hatte der Kläger das Writ ausgewählt, wurden die fallspezifischen Daten eingetragen, und nachdem der Kläger die teils sehr hohe Gebühr bezahlt hatte, ${ }^{52}$ wurde das Writ gesiegelt und dem zuständigen Gerichtsherren zugestellt. ${ }^{53}$ Nur in Ausnahmefällen erfolgte die Zustellung direkt an den Beklagten. Vielmehr oblag es normalerweise dem Gerichtsherren, den Beklagten vorzuladen, indem er ihm das Writ überbrachte. Diese Vorladung hatte der Gerichtsherr der Chancery im sogenannten Return of the Writ zu bestätigen. ${ }^{54}$

\section{cc. Bedeutung der Writs für das Verfahren}

Die Wahl des Writs entschied, nach welchen Verfahrensregeln der Prozess stattzufinden hatte. Nach dem ausgewähltem Writ bestimmte sich die Zuständigkeit des Gerichts, die Art und Weise der Vorladung, die Rechtsfolgen bei Säumnis, die Fristen für die Ansetzung des Termins, die Anforderungen an die Parteivorträge (Pleadings), die einschlägigen Beweismittel (Eid, Zweikampf, Geschworene) usw. Der gesamte Ablauf des Prozesses von der Ladung bis zur Vollstreckung hing demnach von der Wahl des Writs ab. ${ }^{55}$ Die Wahl des Writs hatte mithin elementare Bedeutung für das gesamte Verfahren.

\section{dd. Bedeutung der Writs für das materielle Recht}

Zumindest ebenso bedeutend wie der Einfluss des Writs auf das Verfahren war die Bedeutung des Writs für das materielle Recht. Das Original Writ entschied, nach welchen materiellen Rechtsgrundsätzen der Richter über den ihm vorgetragenen Sachverhalt urteilte.

Dabei hatten die Parteien nur den Sachverhalt vorzutragen. Es war Sache des Richters, die einschlägigen Rechtssätze ${ }^{56}$ zu finden und auf den Sachverhalt anzu-

\footnotetext{
${ }^{49}$ Schulz, 54 (1942) Juridical Review, S. 12

${ }^{50}$ Holdsworth, History I, S. 421

${ }^{51}$ Pollock/Maitland, I, S. 211

${ }^{52}$ Pollock/Maitland, II, S. 151

${ }^{53}$ Peter, S. 29

${ }^{54}$ Peter, S. 29

${ }^{55}$ Maitland, Forms of Action, S. 4; Pollock/Maitland, II, S. $568 \mathrm{ff}$.

${ }^{56}$ Diese Rechtssätze stellen sich gerade nicht als abstrakte Normen dar, die den Anspruch erheben, auf alle Fälle anwendbar zu sein. Dabei handelt es sich um eine Eigenheit des Common Law, die bis heute gültig ist. Der Common Law Jurist ist gerade nicht auf abstrakt formulierte Normen und Gesetzestexte angewiesen, diese sind ihm eher fremd. Er bedient sich in der Regel auch keiner Surrogate. Die Aussage Peters, (S. 36 Fn. 126) Blackstones Commentaries on the Laws of England seien ein Surrogat für ein Zivilgesetzbuch, zeugt insofern von zu „,kontinentaljuristischem“ Denken. Vgl. auch Nicholas, IVRA 9 (1958), S. 235, 236 f..
} 
wenden. ${ }^{57}$ Diese Rechtssätze waren im Laufe der Zeit als Gerichtsgebrauch der Common Law Gerichte für jeden einzelnen Klagetyp entwstanden und entwickelten sich ständig fort. Welche Rechtsnormen angewandt wurden, entschied sich also nach dem Wortlaut des Original Writ, welches der Kläger auszuwählen hatte. Diese Auswahl stellte sich als ausgesprochen schwierig dar. Ansprüche, die nach kontinentaljuristischem Verständnis schuldrechtlicher Art sind, konnten bzw. mussten mit unterschiedlichen Klagen geltend gemacht werden: Genau bestimmte Geldsummen musste der Kläger mit dem Writ of debt geltend machen. ${ }^{58}$ Tatbestandliche Voraussetzung war dabei eine erbrachte Gegenleistung des Klägers $^{59}$ (consideration, quid pro quo). ${ }^{60}$ Entsprach die geforderte Summe nicht genau der, die der Richter für korrekt hielt, wurde die Klage insgesamt abgewiesen. ${ }^{61}$ Stützte sich der Kläger auf eine unterzeichnete und gesiegelte Privaturkunde (deed), musste er mit Hilfe des Writ of convenant vorgehen. ${ }^{62}$ Dabei war es auch möglich, bei Klageeinleitung noch unbezifferten Schadensersatz (unliquidated damages) einzuklagen. ${ }^{63}$ Ließ sich der Sachverhalt weder unter das eine, noch unter das andere Writ fassen, blieb dem Kläger allenfalls die Möglichkeit, die Klage mit dem Writ of trespass on the case einzuleiten. ${ }^{64}$

Die tatbestandlichen Unterschiede ergaben sich nicht aus dem Wortlaut der Writs, sondern aus den von den Gerichten dazu entwickelten Grundsätzen. Dabei gingen die Gerichte sehr restriktiv vor. Ließ sich der Sachverhalt nicht exakt unter das Writ fassen, wurde die Klage abgewiesen. Dafür reichte es oft aus, dass das Gericht zu der Überzeugung kam, dass der vorgetragenen Sachverhalt vom tatsächlichen abwich. Diese oft nur minimalen Abweichungen konnten dazu führen, dass die Klage abgewiesen wurde, weil ein anderes Writ die richtige Klage eingeleitet hätte. ${ }^{65} \mathrm{Ob}$ das abweisende Urteil Rechtskraft entwickelte, lässt sich heute nicht mehr feststellen. Für den Fall, dass keine Rechtskraftwirkung eintrat, war der Kläger jedoch gezwungen, ein neues Writ zu beantragen und die oft hohen Kosten zu tragen, abgesehen von der Tatsache, dass sich der Prozess erheblich in die Länge zog. ${ }^{66}$ Dabei bestand das Risiko, dass das neue Writ auch nicht zum Erfolg führte. Bis ins 14. Jahrhundert schaffte die Chancery diesem Problem durch Schaffung neuer Writs Abhilfe. ${ }^{67}$ Im 14. Jahrhundert erstarrte das Writ-System jedoch allmählich, so dass die Missstände deutlicher zu Tage traten.

Festzuhalten bleibt, dass das gesamte Privatrecht nicht nach materiellen Anspruchsgrundlagen, sondern nach prozessualen Rechtsbehelfen geordnet war. Insoweit entwickelte sich das Common Law auch durch die Schaffung und den Gebrauch neuer Writs. Dieses aktionenrechtliche Denken im engeren Sinne ${ }^{68}$ ist dem

\footnotetext{
${ }^{57}$ „Iura novit curia“. Vgl. auch Peter, S. 36.

${ }^{58}$ Peter, S. 36

${ }^{59}$ Insofern stellt diese Unterscheidung die Grundlage der „consideration-Doktrin“ dar, wonach nach Common Law vertragliche Ansprüche nur bei erbrachter Gegenleistung einklagbar sind, es sei denn, der Kläger kann sich auf ein deed stützen. Vgl. Zweigert/Kötz, S. 384

${ }^{60}$ Holdsworth, III, S. $420 \mathrm{ff}$.

${ }^{61}$ Blackstone, III, S. 155; Holdsworth, III, S. 423 f.

${ }^{62}$ Fitzherbert/Hale, S. 145 A ff.

${ }^{63}$ Holdsworth, III, S. 417

${ }^{64}$ Fitzherbert/Hale, S. 92 E ff.

${ }^{65}$ Potter, S. 287 f.

${ }^{66}$ James, S. 28; Maitland, Forms of Action, S. 4.

${ }^{67}$ Peter, S. 37

${ }^{68}$ Wieacker, Privatrechtsgeschichte, S. 187
} 
Common Law Juristen bis heute eigen ${ }^{69}$ und findet sich historisch sonst nur in den honorarischen Klagen des römischen Amtsrechts. ${ }^{70}$

\section{ee. Einschränkung der Befugnis der Chancery zur Herausgabe neuer Writs im 13. Jahrhundert}

Im 13. Jahrhundert verstärkte sich die Opposition des Adels gegen die Macht des Kanzlers, mit Hilfe neuer Writs den Lehnsherren die Jurisdiktionsgewalt zugunsten der Königsgerichte zu entziehen. In der Magna Charta wurde 1215 festgelegt, dass das Writ praecipe quod reddat nur noch für Kronvasallen verwendet werden durfte. $^{71}$ D. h., nur wenn der Kläger behauptete, sein Lehen direkt vom König erhalten $\mathrm{zu}$ haben, konnte er ein Writ praecipe quod reddat erwirken. Diese Beschränkung wurde durch die Entwicklung des Writ of novel disseisin, als rein possessorische Klage, und der Writs of entry alsbald umgangen. ${ }^{72}$ Damit konnten alle möglichen Rechtsstreitigkeiten vor die Königsgerichte gezogen werden, ohne dass es darauf ankam, ob es sich um ein Kronlehen handelte. Der Kläger musste lediglich behaupten, der Beklagte habe das Land wegen eines kürzlich eingetretenen rechtswidrigen Umstandes erhalten. ${ }^{73}$

In der Mitte des 13. Jahrhunderts wurden die Befugnisse der Chancery weiter eingeschränkt. ${ }^{74}$ Insbesondere in den Provisions of Oxford wurde 1258 beschlossen, dass neue Writs nur noch mit Zustimmung des magnum concilium, welches aus Kronvasallen bestand, erlassen werden konnten. ${ }^{75}$ Unter Edward I (1272-1307) begann die ständige Beteiligung des Parlaments bei der Rechtsetzung durch die Schaffung von Statutes. ${ }^{76}$

Das Statute von Westminster II von 1285 regelte die Ausstellung neuer Writs grundsätzlich. ${ }^{77}$ Neue Original Writs durften prinzipiell nur mit Zustimmung des Parlaments erlassen werden. ${ }^{78}$ Der Chancery blieb jedoch die Möglichkeit, bei

\footnotetext{
${ }^{69}$ Nicholas, IVRA 9 (1958), S. 235, 236

${ }^{70}$ Behrends, JuS 1985, S. 878, 880

${ }^{71}$ Art. 24 der Magna Charta; Peter, S. 71, 24; Maitland, Forms of Action, S. 23 ff.

${ }^{72}$ Maitland, Forms of Action, S. 23 ff., 42; Pollock/Maitland, II, S. 65 f.

${ }^{73}$ Maitland, Forms of Action, S. 42

${ }^{74}$ So im Parliament of Merton 1235-1236 und in den Provisions of Oxford 1258.

${ }^{75}$ Pollock/Maitland, II, S. 196; Potter, S. 283

${ }^{76}$ Peter, S. 72 ff.

${ }^{77}$ Kapitel 24: „Et quotienscumque de cetero evenit in Cancellaria, quod in uno casu reperitur breve et in consimili casu cadente sub eodem iure et simili indigente remedio (scil. non reperitur), concordent clerici de Cancellaria in brevi faciendo vel atterminent querentes in proximo parliamento et scribant casus, in quibus concordare non possunt, et referant eos ad proximum parliamentum et de consensu iurisperitorum fiat breve ne contigat de cetero quod curia diu deficiat querentibus in justitia perquirenda. " - Und wenn immer es von nun an in der Chancery vorkommt, dass in einem Fall ein Writ gefunden wird, in einem anderen ähnlich gelagerten Fall, der nach einem Rechtsbehelf verlangt, aber keiner gefunden wird, dann sollen die Angestellten der Kanzlei zustimmen, das Writ auszustellen oder aber die Klage bis zur nächsten Sitzung des Parlaments aussetzen und die Fälle, in denen sie nicht zustimmen konnten, aufschreiben und auf dem nächsten Parlamentstag vortragen, damit nach Übereinstimmung von Rechtsgelehrten ein Writ ausgestellt wird, damit das Gericht nicht allzu lange den Rechtssuchenden die Gerechtigkeit vorenthält.

${ }^{78}$ Die Bedeutung des Statute of Westminster II wird von verschiedenen Autoren unterschiedlich eingeschätzt. Zum Teil wird das Statute als das endgültige Ende der Befugnis der Chancery zur Ausstellung neuer Writs erachtet, Peter, S. 72 f.. Andere Autoren hingegen interpretieren das Statute genau umgekehrt. Danach wurde die Befugnis der Chancery zur Schaffung neuer Writs, die schon in den Provisions of Oxford 1258 quasi abgeschafft worden war, zu Teilen wieder hergestellt, indem es der Chancery gestattet wurde, Writs in consimili casu auszustellen, Nicholas IVRA 9 (1958), S. 235, 237 f..
} 
geringfügigen Abweichungen von bekannten Writs, Variationen dieser Formulare in consimili casu auszustellen. ${ }^{79}$ Dadurch kam es zu einer Erstarrung des WritSystems insbesondere auf dem Gebiet des Boden- und Lehnsrechts. ${ }^{80}$ Die Möglichkeit, Writs in consimili casu auszustellen, nutzte die Chancery besonders auf dem Gebiet des Vertrags- und Deliktsrechts. ${ }^{81}$ Dazu wurde das Writ of trespass upon the special case entwickelt. Bis 1360 gab es das Writ of trespass nur in den Varianten trespass vi et armis und contra pacem. ${ }^{82}$ Mit der Einführung des Writ of trespass on the special case konnten verschiedenste Rechtsverletzungen eingeklagt werden. Anfangs war die Anwendung von Gewalt noch Voraussetzung, damit man ein Writ of trespass erwirken konnte. So beim Writ of trespass in assault and battery (Verletzung einer Person), Writ of trespass de bonis asportatis (Sachbeschädigung) und Writ of trespass de bonis clausum fregit (Verletzung der Rechte an Land), die alle noch den Zusatz vi et armis oder contra pacem trugen. ${ }^{83}$

Später konnte ein solches Writ auch bei Vertragsverletzungen, Täuschungen durch den Verkäufer oder Verletzungen durch Tiere erwirkt werden. Schließlich wurden sogar Writs of trespass gebräuchlich, bei denen die Rechtsverletzung nicht auf vorsätzlicher Gewaltanwendung beruhte. ${ }^{84}$

Neben diesen bedeutenden Entwicklungen, die das gesamte materielle Vertragsund Deliktsrecht bis heute formen, ${ }^{85}$ erstarrte das Register of Writs, von sprachlich-stilistischen Änderungen abgesehen. ${ }^{86}$

\section{b. Das Pleading-Verfahren im Common Law bis zum 17. Jahrhundert}

Unter Pleadings versteht man im englischen Zivilprozess die mündlichen Vorträge der Parteien. Die Pleadings waren genau bestimmte Wechselreden, durch die der Streitpunkt, über den später Beweis erhoben werden sollte, zwischen den Parteien festgelegt wurde. ${ }^{87}$ Die Pleadings sollen hier nur soweit betrachtet werden, als es um den klägerischen Eröffnungsantrag geht.

Im ersten Termin vor Gericht trug der Kläger seinen Eröffnungsantrag vor, sog. narratio oder count. Dieser Vortrag verfolgte den Zweck, den Sachverhalt zu erläutern sowie den Grund der Klage zu erörtern. Dabei musste sich der Kläger unter weiterer Präzisierung der im Writ enthaltenen Angaben genau an den Wortlaut des Writs halten. ${ }^{88}$

Die besondere Schwierigkeit bestand darin, die narratio korrekt vorzubringen, insbesondere, da der wichtigste Teil des Writs wiederholt werden musste. Jede Klage hatte ihre eigenen Formvorschriften, die eingehalten werden mussten. Unterlief dem Kläger ein Fehler, sei es ein Irrtum oder ein Versprecher, lief er Gefahr, den Fall zu verlieren. ${ }^{89}$

\footnotetext{
${ }^{79} \mathrm{Vgl}$. Maitland, Forms of Action, S. $65 \mathrm{f}$.

${ }^{80}$ Peter, S. 73

${ }^{81}$ Peter, S. 73 unterschätzt insoweit die Bedeutung der Rechtschöpfung der Chancery. Vgl. dazu auch Nicholas, IVRA 9 (1958), S. 235, 237 f.

${ }^{82}$ Maitland, Forms of Action, S. 66

${ }^{83}$ Maitland, Forms of Action, S. 66

${ }^{84} \mathrm{Vgl}$. die Beispiele bei Baker/Milsom, S. 367 und 554 und auch Maitland, Forms of Action, S. $90 \mathrm{f}$.

${ }^{85}$ Nicholas, IVRA 9 (1958), S. 235, 238

${ }^{86}$ Maitland, Collected Papers II, S. 162 ff.; Peter, S. 47; vgl. zur Systematik auch Samuel, ZEuP 3 (1995), S. 375, 395

${ }^{87}$ Bieresborn, S. 423

${ }^{88}$ Baker, Spelman's Reports II, S. 604

${ }^{89}$ Holdsworth, Development, S. 614, 616
} 
Um dieses Risiko zu minimieren, bedienten sich die Parteien seit der Mitte des 13. Jahrhunderts professioneller Vorsprecher, deren Aufgabe es war, die Vorträge richtig und vollständig für die Partei abzugeben. Dies hatte mündlich in französischer Sprache zu geschehen. Der Vortrag wurde dann vom Gerichtsdiener ins Lateinische übersetzt und schriftlich fixiert. ${ }^{90}$ Bis zum Ende des 13. Jahrhunderts setzte es sich in der Praxis durch, dass die Vorträge bis zur schriftlichen Fixierung im Gerichtsprotokoll noch geändert werden konnten. ${ }^{91}$

Das führte zum System des versuchsweisen Vorbringens (tentative pleadings), wobei die Parteien etwas vortrugen, um die Aussicht auf Erfolg auszuloten. Schien das Vorbringen keinen Erfolg zu versprechen, wurde der Vortrag zurückgenommen, ohne dass dies weitere Folgen gehabt hätte. ${ }^{92}$ Die Pleadings mussten klar und vollständig sein und ausschließlich präzise Tatsachen enthalten, so dass ein eindeutiger Streitpunkt festgelegt werden konnte. ${ }^{93}$ Dabei musste der klägerische Vortrag den Gegenstand des Streits unter genauester Angabe der damit zusammenhängenden Fakten, wie z.B. Zeitpunkt und Ort des Kaufs, Diebstahls usw., Wert, Preis der Sache usw., enthalten. Diese Angaben hatte der Kläger anschließend zu beschwören. ${ }^{94}$ Machte er eine erhebliche Angabe nicht, war sein Vortrag unzureichend und damit angreifbar, selbst dann, wenn der Kläger mangels Kenntnis nicht in der Lage war, die Angabe zu machen. ${ }^{95}$ Er musste also Angaben erfinden, um das Pleading wasserdicht zu machen, wohl wissend, dass er die Angaben nicht würde beweisen müssen. ${ }^{96}$ Es handelte sich grundsätzlich um eine Form des Bestimmtheitsgrundsatzes, die in ihrer praktischen Handhabung diesen Grundsatz ad absurdum führte. Darüber hinaus galt die Verhandlungsmaxime. Gegenstand der Entscheidung war nur, was zwischen den Parteien streitig war. $^{97}$

Im Laufe der Zeit erwiesen sich die mündlichen Pleadings als unpraktikabel, da der Sinn der Pleadings oft verfehlt wurde. Die rituellen Vorträge der Vorsprecher beschleunigten die Präzisierung des Streitpunktes nicht, und den Parteien blieben diverse Möglichkeiten, den Prozess zu verschleppen. ${ }^{98} \mathrm{Im}$ 14. und 15. Jahrhundert wurde es möglich, einen schriftlichen Entwurf für den Vortrag bei Gericht einzureichen. ${ }^{99}$ Die Einführung schriftlicher Pleadings änderte jedoch nicht, dass der Rechtsstreit vor Gericht mündlich verhandelt wurde. ${ }^{100}$

\section{Klageeinleitung im Equity-Verfahren}

Die Equity ${ }^{101}$ entwickelte sich seit dem 13. Jahrhundert als zweiter großer Zweig des englischen Zivilrechts parallel zum Common Law. Dabei war sie nicht nur durch abweichende materielle Regeln, sondern auch durch ein eigenständiges Verfahren gekennzeichnet.

\footnotetext{
${ }^{90}$ Baker, Intrduction, S. 93

${ }^{91}$ Baker, Intrduction, S. 93

${ }^{92}$ Ausführlich zum System des versuchsweisen Vorbringens (tentative pleading): Bieresborn, S. 441.

${ }^{93}$ Holdsworth, History III, S. 633

${ }^{94}$ Holdsworth, History IX, S. 285 f.

${ }^{95}$ Holdsworth, History IX, S. 319

${ }^{96}$ Holdsworth, History IX, S. 319

${ }^{97}$ Baker, S. $91 \mathrm{f}$.

${ }^{98}$ Milsom, S. 71

${ }^{99}$ Holdsworth, History III, S. 641

${ }^{100}$ Potter, S. 337

${ }^{101}$ Der Begriff kommt vom lateinischen aequitas und bedeutet soviel wie Billigkeit.
} 


\section{a. Entwicklung der Equity-Rechtsprechung}

Für die Entstehung der Equity zeichneten insbesondere zwei Missstände des Common Law verantwortlich. Erstens lief der Kläger aufgrund der Formstrenge und der Eigenarten des Writ-Verfahrens Gefahr, sein Recht nicht durchsetzen zu können. Sei es, weil kein exakt passendes Writ existierte ${ }^{102}$ oder weil der Kläger beispielsweise eine zu hohe Summe eingeklagt hatte, so dass die gesamte Klage abgewiesen wurde, obwohl dem Kläger ein Teil der Summe zustand. ${ }^{103}$ Zweitens trat das materielle Recht hinter den Verfahrensfragen so weit in den Hintergrund, dass fast nur noch über das Verfahren gestritten wurde. ${ }^{104}$ Dies wog um so schwerer, als dass es keine Appellationsinstanz gab, die das Urteil überprüfen und gegebenenfalls ersetzen konnte. Der unterlegenen Partei blieb nur die Möglichkeit, mit Hilfe eines Writ of error das Gerichtsprotokoll auf Irrtümer hin überprüfen zu lassen. In solchen Fällen konnte das Urteil von einem Gericht mit Jurisdiktionsgewalt für Irrtumsfälle ${ }^{105}$ aufgehoben und an das erstinstanzliche Gericht mit bindender Weisung zurückverwiesen werden. ${ }^{106}$ Es wurde ausschließlich das Gerichtsprotokoll überprüft. Mögliche Appellationsgründe ergaben sich aber oft gerade nicht aus dem Protokoll, so dass dieser Weg meist erfolglos blieb. ${ }^{107}$

Seit dem 13. Jahrhundert wandten sich Kläger zum Teil direkt an den König ${ }^{108}$, der seinem Eid ${ }^{109}$ nach verpflichtet war, Gerechtigkeit zu üben. ${ }^{110}$ Der König hatte aber nach allgemeiner Auffassung seine Rechtsprechungsmacht mit Schaffung der Gerichte erschöpft, so dass alle Bitten mit dem Hinweis abgewiesen wurden, der Antragsteller solle bei einem Common Law Gericht Klage einreichen, es sei denn, das Common Law wäre im speziellen Fall lückenhaft. ${ }^{111}$

Ebenso wurden Eingaben an den König gebräuchlich, in denen sich die Bittsteller über eine ungerechte Behandlung durch die Common Law Gerichte beklagten. Diese Petitionen wurden im 14. Jahrhundert so üblich, dass ihretwegen der Königsrat außerordentlich tagte. ${ }^{112}$ Im folgenden kam es zu zwei verschiedenen Verfahren. Regte die Petition eine Änderung des materiellen oder des Verfahrensrechts an, wurde sie vom Parlament beschieden und bei Stattgabe zur Gesetzesform erhoben. ${ }^{113}$ Handelte es sich um private Rechtsstreite, wurden die Angelegenheiten an einzelne Mitglieder des Königsrates delegiert, wie den Chancellor,

\footnotetext{
${ }^{102}$ Da seit dem Statute von Westminster II praktisch kaum noch neue Writs geschaffen wurden, führte dies zu erheblichen Lücken im Rechtssystem. Siehe dazu oben B.I.2.a.ee.

${ }^{103}$ Curzon, S. 88; Ein umgekehrtes Beispiel materieller Ungerechtigkeit bietet der Fall Denom v. Scott 17 aus dem Jahr 1343. Obwohl der Beklagte, der auf Zahlung von 20 Pfund verklagt worden war, beweisen konnte, dass er die Schuld beglichen hatte, wurde er zur erneuten Zahlung verurteilt, da die Schuldurkunde bei der ersten Zahlung nicht vernichtet worden war. Shardelow J.: „, The court adjudges that the plaintiff do recover his debt and damages assessed by the court. And see now, that the deed is cancelled. - Das Gericht urteilt, dass der Kläger Schuld und Schadensersatz liquidieren kann. Der Beklagte soll dafür Sorge tragen, dass die Urkunde vernichtet wird“", Zitat aus Stubbs, S. 298

${ }^{104}$ Curzon, S. 88; James, S. 28 f.

${ }^{105} \mathrm{Sog}$. jurisdiction in error.

${ }^{106}$ Baker, Introduction, S. 157; Sim/Pace, S. 27

${ }^{107}$ Erst 1830 wurde eine Court of Exchequer Chamber in den Jucicature Acts zur Appellationsinstanz für alle drei Common Law Gerichte erhoben.

${ }^{108}$ Sog. bills; vgl. Potter, S. 572 f..

${ }^{109}$ Im Krönungseid Edwards II hieß es: ,...to do equal and right justice and discretion in mercy and truth..."; vgl. Statutes of the Realm, Vol. I, S. 168; Baker, Introduction, S. 113

${ }^{110}$ Caenegem, History, S. 44

${ }^{111}$ Baker, S. 113

${ }^{112}$ Baker, S. 113

${ }^{113}$ Baker, S. 113
} 
Admiral oder Marshall, an den sich der Bittsteller dann zu wenden hatte. ${ }^{114}$ Aus diesem Verfahren entwickelten sich verschiedene Sondergerichte. ${ }^{115}$ Herausragenden Einfluss auf das englische Recht hatte allerdings die Chancery mit der Entwicklung der Rechtsprechung in equity. ${ }^{116}$

Die Chancery hatte ursprünglich als königliche Kanzlei die Aufgabe, die königlichen Befehle zu verfassen. Insbesondere stellte sie Writs aus. ${ }^{117}$ Mit steigender Zahl der Bittgesuche an den König erweiterte sich ihr Aufgabenkreis. Anfänglich leitete die Chancery die Entscheidungen des Königs an die Instanzgerichte weiter, damit diese unter Berücksichtigung der Rechtsauffassung des Königs erneut entschieden. In der zweiten Hälfte des 14. Jahrhunderts wurde es aufgrund der hohen Zahl an Bittgesuchen üblich, diese direkt an die Chancery zu richten. Die Chancery war mit hohen kanonistisch und römisch geschulten Beamten besetzt, ${ }^{118}$ die ohnehin über die Ausstellung der Writs entschieden, so dass sie als geeignete Stelle zur Schaffung neuer Rechtsbehelfe erschien. ${ }^{119}$ Im Laufe der Zeit setzte sich die Ansicht durch, niemand solle die Chancery ohne Rechtsbehelf verlassen. ${ }^{120}$

Da jedoch ab 1258 nicht ohne weiteres neue Writs erlassen werden konnten, ${ }^{121}$ wurden ab dem 14. Jahrhundert privatrechtliche Streitigkeiten von der Chancery direkt entschieden. ${ }^{122} \mathrm{Ab}$ dem 15. Jahrhundert geschah dies sogar im eigenen Namen, ${ }^{123}$ nicht mehr im Namen des Königs. ${ }^{124}$

\section{b. Das Verfahren der Equity-Rechtsprechung}

In Equity-Sachen vor der Chancery wurde ein Verfahren angewandt, das sich vom Common Law-Verfahren wesentlich unterschied. ${ }^{125}$ Der Chancellor war Richter, der über Tatsachenfragen ebenso entschied, wie über Rechtsfragen, ohne dass dabei eine Jury tätig wurde. ${ }^{126}$

\footnotetext{
${ }^{114}$ Baker, S. 113 f.; Bieresborn, S. 451

${ }^{115}$ Das waren neben der Chancery, die die Equity-Rechtsprechung entwickelte, insbesondere die geistlichen Gerichte sowie der Court of Admiralty, der sich mit allen Rechtsstreitigkeiten im Zusammenhang mit der Seefahrt beschäftigte. Beide Gerichte wandten das römisch-kanonische Verfahren an. Vgl. ausführlich dazu Bieresborn, S. $465 \mathrm{ff}$.

${ }^{116}$ Noch heute wird in Urteilen zum Teil unterschieden, ob dem Kläger einen Anspruch at law, d. h. nach Common Law, oder in equity zusteht.

${ }^{117}$ Bezüglich dieses originären Aufgabenbereichs spricht man auch von der Latin side, da Aufzeichnungen über das Common Law, insbesondere die Ausstellung der Writs in lateinischer Sprache erfolgte. Baker, S. 116; Curzon, S. 103

${ }^{118}$ Koschaker, S. 213 ff.; Wieaker, S. 105 Fn. 22

${ }^{119}$ Baker, S. 117

${ }^{120}$ Nullus recedat a curia cancellariae sine remidio. - Niemand verlässt die Chancery ohne Rechtsbehelf. Baker, S. 117

${ }^{121}$ S. o. B.I.2.a.ee.

${ }^{122}$ Insoweit spricht man auch von der English side, obwohl die Aufzeichnungen über die Billigkeitsrechtsprechung bis zum 16. Jahrhundert auf Rechtsfranzösisch und erst danach auf Englisch verfaßt wurden. Baker, S. 117; Curzon, S. 103

${ }^{123}$ Baker, S. 117; dazu: Holmes, S. 705

${ }^{124}$ Die damit vorprogrammierten Kompetenzkonflikte zwischen Common Law und Equity wurden grundlegend im Fall des Earl of Oxford ((1615) 1 Ch. Rep. 6) dahingehend gelöst, dass das Equity-Verfahren nur eingreifen sollte, wenn kein ausreichender Rechtsbehelf nach Common Law zur Verfügung stand. War die Equity jedoch anwendbar, hatte sie Vorrang vor dem Common Law. „Equity shall prevail"; ,Equity follows the Law“; Curzon, S 108 f.; Holdsworth, V, S. 235 f.

${ }^{125}$ Maitland, Essays, S. 133: ,...certain it is that [the clerical chancellors] borrowed the main principles of their procedure from the canonists.“ - ,... es ist sicher, dass die geistlichen Kanzler Grundprinzipien ihres Verfahrens von den Kanonisten entliehen."

${ }^{126}$ Caenegem, History, S. 45
} 
Somit wurde der Streit in jeder Hinsicht von einem geschulten Juristen entschieden. Dabei oblagen dem Chancellor mehr Aufklärungspflichten als im Common $L a w$-Verfahren. Er hatte den Rechtsstreit vollständig auszuforschen, um eine billige Entscheidung treffen zu können. Das umfasste auch eine richterliche Parteien- und Zeugenvernehmung. ${ }^{127}$ Trotzdem galt auch im Verfahren vor der Chancery die Verhandlungsmaxime, d.h. die Parteien bestimmten den Streitstoff. ${ }^{128}$

Darüber hinaus gestaltete sich das Equity-Verfahren relativ formfrei, was sich als Konsequenz aus der Tatsache ergab, dass die Equity gerade erst aufgrund der Probleme entstanden war, die die Formstrenge des Common Law-Verfahrens verursachte. ${ }^{129}$

\section{aa. Writ of subpoena}

Die Einleitung des Equity-Verfahrens unterschied sich von der des Common LawVerfahrens vor allem durch die Tatsache, dass es bei der Equity nur ein Writ gab, das sog. Writ of subpoena. Damit wurde der Beklagte unter Androhung einer Strafe von Amts wegen vor die Chancery geladen. ${ }^{130}$ Das Writ of subpoena hatte mithin keine materiellrechtliche Bedeutung, sondern diente ausschließlich der Ladung des Beklagten. Es handelte sich also um ein Juridical Writ, während die Writs zur Einleitung eines Common Law-Verfahrens Original Writs waren. ${ }^{131}$

\section{bb. Bill of complaint}

Während anfangs eine mündliche Beschwerde bei der Chancery zur Erwirkung eines Writ of subpoena ausreichte ${ }^{132}$, wurde im Laufe der Zeit die schriftliche Eingabe eines sog. Bill of complaint die Regel. ${ }^{133}$ Frühe Bills of complaint beinhalteten einen formlosen Antrag auf Erlass eines Writ of subpoena sowie eine knappe Mitteilung der Gründe für den Antrag. Dabei war der Inhalt nicht so streng vorgegeben wie der Wortlaut beim Original Writ, was sich aus der Entstehungsgeschichte der Equity erklärt. ${ }^{134}$ Später entwickelte sich ein Muster, nach dem die Bills of complaint gefasst wurden. Damit sollte sichergestellt werden, dass bestimmte Elemente vorhanden waren. Dies waren insbesondere die Namen der Beteiligten, die Beschwer des Klägers, das angegriffene Verhalten des Beklagten und der daraus entstandene Schaden, die Feststellung, dies widerspräche der Billigkeit sowie die Bitte an das Gericht, das Verfahren durchzuführen. ${ }^{135}$ Die Reihenfolge der Elemente änderte sich bis zum 17. Jahrhundert. Teilweise kam es zu inhaltlichen Vermischungen. ${ }^{136}$

\footnotetext{
${ }^{127}$ Baker, S. 119; Potter, S. 581 f.

${ }^{128}$ Engelmann/Millar, S. 19 f.

${ }^{129}$ Y.B. 9 Edw. 4, Trin.pl. 9: ,En le Chancery fuit touche per le Chancellor, que home ne serra prejudice, per mispleder, ou pur defaut de fourme, mes solonqs la verity de son matter, nous avomus adjudger secundum conscientam, \& non secundam allegata... " - In der Chancery wird durch den Chancellor so gehandelt, dass niemand wegen eines Fehlers beim Vortrag oder eines bloßen Formfehlers vorverurteilt wird, sondern sein Fall allein auf Grundlage der Wahrheit entschieden wird, weil wir aufgrund des Bewußtseins urteilen, nicht aufgrund von Vorgetragenem...

${ }^{130}$ Baker, S. 119, ein Beispiel dazu ebda. S. 624.

${ }^{131}$ Baker, S. 119

${ }^{132}$ Baker, S. 119

${ }^{133}$ Potter, S. 579

${ }^{134} \mathrm{Oft}$ war die Anrufung der Chancery nur erforderlich, weil das Common Law aufgrund von übertriebenem Formalismus keinen Rechtsschutz gewährte. S. o. B.I.3.a.

${ }^{135}$ Holdsworth, IX, S. $379 \mathrm{f}$.

${ }^{136}$ Holdsworth, IX, S. $381 \mathrm{f}$.
} 
Die Bill of complaint stellte neben dem Antrag auf Erlass des Writs of subpoena auch den ersten klägerischen Vortrag dar ${ }^{137}$, der dem Beklagten in Abschrift bei Erscheinen vor Gericht ausgehändigt wurde ${ }^{138}$, damit dieser darauf erwidern konnte. Daher enthielt die Bill of complaint einen vollständigen Tatsachenvortrag inklusive Beweisangebot. ${ }^{139}$

Über welchen Sachverhalt letztendlich Beweis erhoben und entschieden wurde, ergab sich ausschließlich aus dem klägerischen Vortrag in der Bill of complaint und dem Verhalten des Beklagten, der durch Bestreiten oder Nichtbestreiten die Beweisbedürftigkeit festlegte. ${ }^{140}$

\section{Geistliche Gerichte und Court of Admiralty}

Neben den Common Law Gerichten und der Equity Rechtsprechung befassten sich noch weitere englische Gerichte mit Zivilsachen und entwickelten dabei auch abweichende Verfahrensregeln, die das heutige englische Zivilverfahren mitgeprägt haben.

\section{a. Geistliche Gerichtsbarkeit}

$\mathrm{Ab} 1070$ entwickelten sich in England eigenständige geistliche Gerichte ${ }^{141}$, die insbesondere für weltliche Streitigkeiten Geistlicher untereinander sowie Ehesachen und Testamentsstreitigkeiten zuständig waren. ${ }^{142}$ Bis zur Reformation waren die geistlichen Gerichte Englands Teil der grenzüberschreitenden geistlichen Rechtsprechung mit dem Papst als höchster Instanz. ${ }^{143}$ Mit der Abspaltung der anglikanischen Kirche von Rom 1534 wurde auch das Einlegen von Rechtsmitteln zum Papst abgeschafft. ${ }^{144}$

Allerdings kam es nicht zu einer Verschmelzung der geistlichen Gerichte mit den Common Law Gerichten. ${ }^{145}$ Beachtung verdienen die geistlichen Gerichte insbesondere, weil vor ihnen römisch-kanonisches Verfahrensrecht galt. Dies änderte sich auch nicht, als sich die anglikanische Kirche von Rom löste. Vielmehr blieb das Verfahren vor den geistlichen Gerichten vom Common Law unbeeinflusst. ${ }^{146}$ Die zivilrechtliche Zuständigkeit der geistlichen Gerichte wurde erst 1857 endgültig eliminiert. ${ }^{147}$

\section{b. Court of Admiralty}

Weitaus wichtiger für die Entwicklung des englischen Zivilprozesses als die geistlichen Gerichte, aber von diesen stark beeinflusst, war der Court of Admiralty. Der Court of Admiralty entwickelte sich als eigenständiger Gerichtszweig aus der Curia Regis. ${ }^{148}$ Die ordentlichen Gerichte waren für Streitigkeiten, die mit Schifffahrt zusammenhingen nicht zuständig, da dort Jurys urteilten, deren Mitglieder

\footnotetext{
${ }^{137}$ Holdsworth, IX, S. 379

${ }^{138}$ Holdsworth, IX, S. 340

${ }^{139}$ Hartwieg, S. 54; Holdsworth, IX, S. 379 f.

${ }^{140}$ Caenegem, History, S. 74

${ }^{141}$ Baker, S.147

${ }^{142}$ Holdsworth, I, S. $601 \mathrm{f}$.

${ }^{143}$ Baker, S. 147

${ }^{144}$ Baker, S. 151; Stubbs, S. 276

${ }^{145}$ Baker, S. 151; Bieresborn, S. 469

${ }^{146}$ Langdell, S. 753, 766

${ }^{147}$ Statute 18 \& 19 Vict.,c.41; 20 \& 21 Vict., c. 77 und c.85; danach ging auch die Zuständigkeit für Ehe- und Erbschaftssachen auf die weltlichen Gerichte über.

${ }^{148}$ Holdsworth, I, S. 545
} 
wohnen mussten, wo der Streit entstanden war. ${ }^{149}$ Die meisten Probleme, die mit der Seefahrt zu tun hatten, wie unerlaubte Handlungen oder Vertragsschlüsse, entstanden aber gerade außerhalb des Landes, weshalb die ordentlichen Gerichte keine Jurisdiktionsgewalt hatten. Diese Zuständigkeitslücke füllte zunächst die Curia Regis, später der Court of Admiralty aus.

Das Gericht weitete seine Kompetenz sukzessive auf alle Zivilsachen aus, die im Ausland ihren Entstehungsgrund hatten und in irgendeinem Kontext zur Seefahrt standen, was sich aufgrund der geographischen Lage Englands bei allen internationalen Zusammenhängen konstruieren ließ. Dieser Umstand führte dazu, dass sich der Court of Admiralty zu einer Art europäischem Handelsgericht entwickelte. $^{150}$

Dies ist um so bedeutender, da vor dem Court of Admiralty das römisch-kanonische Verfahren angewandt wurde, das sonst nur die geistlichen Gerichte praktizierten, ${ }^{151}$ was zum einen daran lag, dass das Common Law-Verfahren mangels Jury inadäquat war. Zum anderen waren viele Richter am Court of Admiralty im römisch-kanonischen Recht geschult und wandten deshalb dieses Verfahren anwandten. ${ }^{152}$ Diese Praxis erscheint um so schlüssiger, wenn man sich vor Augen führt, dass ein großer Teil des englischen materiellen Seerechts auf römischen Rechtsprinzipien beruhte ${ }^{153}$, da bei internationalen Sachverhalten oft völkerrechtliche Grundsätze eine Rolle spielten. ${ }^{154}$

Die Jurisdiktionsgewalt des Court of Admiralty wurde zu Beginn des 17. Jahrhunderts stark eingeschränkt, als entschieden wurde, dass er nur noch für Streitigkeiten zuständig sein sollte, die auf See entstanden waren, nicht jedoch für im Ausland geschlossene Verträge. ${ }^{155}$ Mitte des 19. Jahrhunderts wurde die Zuständigkeit des Gerichts zwar wieder erweitert, 1875 erfolgte dann aber die Eingliederung in die Common Law Gerichte. ${ }^{156}$

\section{Zivilprozessrechtsreformen im 17. und 18. Jahrhundert}

Die Reformen des 17. und 18. Jahrhunderts betrafen sowohl das Verfahren nach Common Law als auch der Equity. Im Common Law änderten sich insbesondere die Regeln zum Pleading und zur Säumnis. ${ }^{157}$ So wurde insbesondere das Recht des versuchsweisen Vorbringens (tentative pleadings) abgeschafft. ${ }^{158}$ Das führte dazu, dass die Parteien alle Tatsachen im ersten Pleading vorbringen mussten, um nicht zu riskieren, dass ein späterer Vortrag als unzulässige Änderung nicht zugelassen wurde. ${ }^{159}$

In der Equity-Rechtsprechung kam es ebenfalls zu Änderungen. Diese betrafen unter anderem die Bill of complaint. Hatte die Bill of complaint bis zum 16. Jahr-

\footnotetext{
${ }^{149}$ Baker, S. 141; Potter, S. 190 ff

${ }^{150}$ Holdsworth, I, S.552; Zimmermann, ZEuP 1 (1993), S. 31 f.

${ }^{151}$ Blackstone, Commentaries III S. 69: ,Its proceedings are according to the methods of the civil law, like those of the ecclesiestcal courts... “- Sein Verfahren bestimmt sich nach den Methoden (Regeln) des Zivilrechts (im Gegensatz zum Common Law), wie das Verfahren der geistlichen Gerichte.

${ }^{152}$ Zimmermann, ZEuP 1 (1993), S. 31 f.

${ }^{153}$ Daneben spielten im englischen Seerecht die Rechte von Oleron und von Rhodos eine wichtige Rolle. Vgl. Zimmermann, a.a.O. S. 29.

${ }^{154}$ Holdsworth, V, S. $99 \mathrm{f}$.

${ }^{155}$ Constable's Case (1601) 5 Co. Rep. 106; Holdsworth, I, S. 557; Zimmermann, a.a.O. S. 32

${ }^{156}$ Potter, S. 204;

${ }^{157}$ Dazu ausführlich Bieresborn, S. $478 \mathrm{ff}$.

${ }^{158}$ Baker, S. 97

${ }^{159}$ Baker, S. 97
} 
hundert noch aus einem einfachen Sachverhaltsvortrag inklusive Namen und Adressen der Parteien bestanden, ${ }^{160}$ machte die große Anzahl an Verfahren vor der Chancery weitergehende Spezifikationen in der Bill of complaint erforderlich. ${ }^{161}$ Zusätzlich zum bis dahin erforderlichen Inhalt der Bill of complaint wurde vom Kläger verlangt, nach seiner Sachverhaltsdarstellung bestimmte Fragen -Interrogatories - an den Beklagten zu stellen, ${ }^{162}$ auf die dieser in seiner Answer qualifiziert antworten musste, um nicht Gefahr zu laufen, dass der Inhalt der klägerischen Behauptung als wahr unterstellt wurde. ${ }^{163}$ Jede der gestellten Fragen musste sich aus dem klägerischen Sachverhaltsvortrag ergeben. ${ }^{164}$ Zur Beschleunigung war der Kläger gehalten, in seinem Vortrag auch gleich auf mögliche Einwendungen und Verteidigungen des Beklagten einzugehen und soweit möglich, deren Unbegründetheit darzulegen. ${ }^{165}$

Die Sachverhaltsdarstellung und die damit einhergehenden Behauptungen, die den Interrogatories zu Grunde lagen, waren für den Kläger bindend, d.h. eine Änderung des Vorbringens war insoweit nicht mehr möglich. Stellte sich der Sachverhalt, so wie der Kläger ihn dargestellt hatte, im Laufe des Prozesses als für diesen ungünstig dar, lief er sogar Gefahr, dass sein eigener Vortrag gegen ihn verwendet wurde.

Schließlich wurde eingeführt, schon in der Bill of complaint die Beweismittel anzugeben, die der Kläger zum Beweise seines Vortrages verwenden wollte. ${ }^{166}$

\section{Römisch-kanonische Einflüsse auf das Verfahren}

Das alte mittelalterliche Writ-System weist oberflächlich einige äußerliche Parallelen zum Aktionenwesen des klassischen römischen Rechts auf. Es lässt sich jedoch, von wenigen Ausnahmen abgesehen ${ }^{167}$, nicht beweisen, dass das römische Recht Einfluss auf Entstehung und Entwicklung des Writ-Systems hatte. ${ }^{168}$

Das Verfahren vor Gerichten des Common Law war auch praktisch keinen Einflüssen durch das römisch-kanonische Verfahren ausgesetzt. Das römisch-kanonische Verfahren wurde hingegen vor dem Court of Admiralty angewandt und beeinflusste das Common Law so indirekt. Darüber hinaus gibt es Parallelen zwischen römisch-kanonischem Prozess und Equity-Verfahren, die auf einen Einfluss schließen lassen.

So entsprachen Bill of complaint und Klaglibell einander funktional weitgehend. Beide stellten den Sachverhaltsvortrag dar, der Grundlage der folgenden Sachentscheidung war. Um beweiserheblich zu werden, musste der Vortrag in der Bill bzw. im Libell vom Beklagten bestritten werden. ${ }^{169}$ Im Gegensatz zum Common $L a w$-Verfahren, wo sich die Parteien auf den Streitpunkt einigen mussten, wurden

${ }^{160}$ S.o. B.I.3.b.bb.

${ }^{161}$ Holdsworth, IX, S. $353 \mathrm{f}$.

${ }^{162}$ Holdsworth, a.a.O. S. 354

${ }^{163}$ Holdsworth, a.a.O. S. 383, 400, 403

${ }^{164}$ Holdsworth, a.a.O. S. 401

${ }^{165}$ Bieresborn, S. 487 f.

${ }^{166}$ Holdsworth, a. a. O. S. $379 \mathrm{f}$.

${ }^{167}$ Ein solche Ausnahme stellt das Writ praecipe quod reddat dar. Siehe dazu oben B.I.2.a.aa.

${ }^{168}$ Den umfangreichsten Versuch macht wohl Peter in „Actio und Writ“. Ein Nachweis von Einflüssen gelingt ihm allerdings nicht. Vielmehr muss er feststellen, dass sich die Ähnlichkeiten von Writ und actio bzw. Register of original Writs und edictum perpetuum in Oberflächlichkeiten erschöpfen. Dazu Broggini, ZRG Rom. 75 (1958), S. 441 ff.; Nicholas, IVRA 9 (1958), S. 235 ff.; Stein, Studia et Documenta 24 (1958), S. 335, der insbesondere auf S. 444 Fn. 10 der Ansicht ist, dass das wenig entwickelte mittelalterliche englische Recht schon aus methodischen Gesichtspunkten gar nicht mit dem hochentwickelten römisch-klassischen Recht vergleichbar ist. ${ }^{169}$ Holdsworth, IX, S. 337 f. 
vor der Chancery in wechselseitigen Vorträgen die beweiserheblichen Streitpunkte festgelegt. Auch dabei handelt es sich um ein System, das dem des römisch-kanonischen Verfahrens entspricht. ${ }^{170}$ Schließlich weist der gemeinsame Wortstamm von Bill und Libell auf eine Verwandtschaft hin. ${ }^{171}$

Die Reformen, die der Equity-Prozess im 17. Jahrhundert erfuhr, lassen auch auf römisch-kanonische Einflüsse schließen. Die Interrogatories ähneln den Positionen des römisch-kanonischen Verfahrens. Auch die Positionen waren bestimmte Tatsachenbehauptungen, auf die der Gegner konkret antworten musste. ${ }^{172}$ In beiden Systemen waren nur bestrittene Tatsachen Gegenstand der Beweiserhebung. Des weiteren stellte die Bindung einer Partei an den eigenen Vortrag, unter Umständen sogar zum eigenen Nachteil, ein Prinzip dar, das dem Common Law fremd $^{173}$, dem römisch-kanonischen Prozess hingegen eigen war (qui-ponit-fatetur-Grundsatz). ${ }^{174}$ Schließlich wurde in beiden Systemen ein ähnliches Maß an Schriftlichkeit gefordert. Bill of complaint und Answer waren genauso schriftlich einzureichen, wie im römisch-kanonischen Verfahren Klaglibell und Exceptionen. ${ }^{175}$

Die Hauptursache für die Beeinflussung der Equity durch das römisch-kanonische Verfahren war die Tatsache, dass die meisten Chancellors bis zum 16. Jahrhundert im römisch-kanonischen Prozess geschulte Kleriker waren. ${ }^{176}$ Dabei übten die Chancellors ihre Rechtsprechungsgewalt als judges of reason and conscience aus ${ }^{177}$ und waren somit in der Entwicklung eigener Verfahrensgrundsätze nicht den Regeln des Common Law unterworfen. Trotzdem stellte die Rechtsprechung in equity nur eine Ergänzung des Common Law dar und war daher gezwungen, technische Begriffe und formale Verfahrensabläufe des Common Law zumindest teilweise zu übernehmen. ${ }^{178}$ Daher kam es nicht zu einer Rezeption des römischkanonischen Prozesses. Das Verfahren vor der Chancery entwickelte vielmehr einen eigenständigen Charakter, ${ }^{179}$ in dem sich Common Law-Grundsätze und Billigkeitserwägungen mit römisch-kanonischen Prinzipien ${ }^{180}$ vereinigten.

\section{Zusammenfassung}

Soweit man Kenntnis vom Verfahren zu angelsächsischer Zeit hat, war ein Zivilprozess im eigentlichen Sinne in England nicht vorhanden. Private Ansprüche wurden in einem Verfahren durchgesetzt, in dessen Mittelpunkt der Schuldvorwurf an den Beklagten stand. Das Verfahren war extrem formalistisch. Die von den Parteien vorzubringenden Vorträge mussten einem genauen Wortlaut folgen. Fehler im Vortrag konnten zum Verlust des Prozesses führen.

Seit dem 13. Jahrhundert entwickelte sich in England mit dem Writ-Verfahren ein aktionenrechtlich geprägtes System, das bis ins 14. Jahrhundert die gesamte Rechtsentwicklung beherrschte. Das Bestehen eines Anspruches hing davon ab,

\footnotetext{
${ }^{170}$ dazu Langdell, Equity-Pleading, S. 753, 757 f.

${ }^{171}$ Potter, S. 572 m. w. N.

${ }^{172}$ Nörr, ZZP 85, S. 160; R. Schmidt, Zivilprozeß, S. 75 für das römische Verfahren.

${ }^{173}$ S.o. B.I.2.b.

${ }^{174}$ Endemann, ZfdZ 15 (1891) S. 177, 245; Langdell, Equity-Pleading, S. 753, 771

${ }^{175}$ Bieresborn, S. 497

${ }^{176}$ Baker, S.114

${ }^{177}$ Coing, L.Q.R. 71, S. 223

${ }^{178}$ Bieresborn, S. 499

${ }^{179}$ Insoweit kann man allenfalls von indirekter Rezeption sprechen. Coing, L.Q.R. 71, S. 231

${ }^{180}$ Coing, L.Q.R. 71, S. 223 ff. weist nach, dass sich die Chancellors bei der Entwicklung des Equity-Verfahrens an der denunciato evangelica des römisch-kanonischen Prozesses orientierten, ohne das System völlig zu übernehmen.
} 
ob ein entsprechendes Writ zur Verfügung stand (No writ, no right.). Das ausgewählte Writ bestimmte auch die prozessualen und materiellen Voraussetzungen des Anspruches. Die Besonderheit des Systems ergab sich gerade aus der fehlenden Trennung von materiellem und Prozessrecht. Die damit einhergehenden Probleme wurden ab dem 14. Jahrhundert deutlich. Insbesondere kam es $\mathrm{zu}$ Rechtslücken, die entstanden, weil für bestimmte Sachverhalte kein Writ vorhanden war oder der extreme Formalismus des Common Law-Verfahrens zu ungerechten Ergebnissen führte. Diesen Phänomenen begegnete das englische Rechtssystem mit der Entwicklung der Equity, die dem Kläger unter gewissen Umständen in equity gewährte, was ihm at (common) law verwehrt worden war.

Das Equity-Verfahren entwickelte seine eigenen Verfahrensregeln, die insbesondere versuchten, die Probleme des Common Law-Verfahrens zu vermeiden. Im Laufe der Zeit wurde jedoch auch das Equity-Verfahren zunehmend rigide und formalistisch.

Beide Verfahren waren nicht kodifiziert, sondern entsprangen Gewohnheitsrecht und Gerichtsgebrauch und beruhten oft auf Präzedenzfällen.

Die Verfahrensgrundsätze unterschieden sich zum Teil erheblich. Im Common Law-Verfahren war die Auswahl des richtigen Writs oft der über Gewinn oder Verlust des Rechtsstreites entscheidende Schritt. In der Equity hingegen wurden alle Fälle mit Hilfe des Writ of subpoena eingeleitet. Dieses Writ beantragte der Kläger mit der Bill of complaint, einem Instrument, das dem Common Law gänzlich fremd war. Die Bill of complaint stellte gleichzeitig den ersten Sachvortrag des Klägers dar, der insbesondere der Sachverhaltsdarstellung diente.

Weil die Zahl der Verfahren in equity stetig anstieg, wurde im 17. Jahrhundert dem Sachverhaltsvortrag in der Bill of complaint bindende Wirkung verschafft. Dies führte zunehmend zur Rigidität des Verfahrens auf einem Gebiet, auf dem das Common Law-Verfahren eher flexible Möglichkeiten bot. Unter dem Common Law konnten die Parteien ihr Vorbringen beliebig ändern, solange sie sich nicht auf den Streitpunkt geeinigt hatten. ${ }^{181}$

Während das Common Law von römisch-kanonischen Einflüssen praktisch unberührt blieb, diente der römisch-kanonische Prozess bei der Entwicklung des Verfahrens in equity teilweise als Vorbild und beeinflusste das Verfahren damit zum Teil erheblich.

${ }^{181}$ Langdell, Equity-Pleading, S. 753, 771 


\section{Von den Reformen des 19. Jahrhunderts bis 1999}

Der erste große Einschnitt, den der Gesetzgeber auf dem Gebiet des Zivilprozessrechts vornahm, waren die Reformen des 19. Jahrhunderts, die das Verfahren bis zur Einführung der Civil Procedure Rules 1998 prägen sollten.

\section{Die Reformen des 19. Jahrhunderts}

Die vorerst meist einschneidende Änderung erfuhr das englische Zivilprozessrecht - und damit einhergehend der Aufbau der Gerichte - in den Reformen des 19. Jahrhunderts. Der Reformbedarf ergab sich, da sich sowohl das Common Law- als auch das Equity- Verfahren so weit verfestigt hatten, dass die Dauer der Prozesse und die extreme Technizität des Verfahrens nicht den Anforderungen entsprachen, die das Industriezeitalter und die damit zusammenhängende $\mathrm{Zu}-$ nahme der Prozesse an sie stellte. ${ }^{182}$

Am Verfahren nach Common Law wurde in erster Linie kritisiert, dass die Gerichte nicht die Suche nach einer materiell gerechten Lösung in den Vordergrund stellten, sondern oft versuchten, die Klage schon an der Auswahl des Writs scheitern zu lassen oder ein Urteil zu fällen, das auf Fehlern in den Pleadings fußte. ${ }^{183}$ Insbesondere enthielt ein klagabweisendes Urteil aufgrund falscher Auswahl des Writs keine Aussage darüber, welches Writ hätte gewählt werden müssen, so dass der Kläger bei erneuter Klage wieder Gefahr lief, mit der gleichen Begründung abgewiesen zu werden. ${ }^{184}$ Dadurch stiegen nicht nur die Dauer der Verfahren, sondern auch die Kosten. ${ }^{185}$

Das Verfahren in equity sah sich der Kritik ausgesetzt, die hohen Anforderungen an Bill of complaint und Answer und insbesondere die Anführung der einzelnen Beweismittel in der Bill würden das Verfahren hemmen und verlangsamen. ${ }^{186}$

\footnotetext{
${ }^{182}$ Bowen, S. 285; Caenegem, History, S. 103

${ }^{183}$ Engelmann/Millar, I, S. 9 f. Nach Holdsworth, IX, S. 308 f. zeigte sich dies insbesondere an der großen Zahl von Entscheidungen, die sich ausschließlich mit Form und Inhalt der Pleadings beschäftigten. Schmitthoff, JZ 1972, S.38, 40 nennt die Entwicklung ein Ausarten ,in eine Art Schachspiel [...], das um seiner selbst willen gespielt wurde“.

${ }^{184}$ Bücker, S. 30

${ }^{185}$ Odgers, S. 220
}

${ }^{186}$ Hartwieg, S. 53; Holdsworth, IX, S. 339 zitiert den berühmten englischen Juristen Jeremy Bentham mit folgenden Worten: „Under the name of a bill, a volume of notorius lies delivered in, with three or four month time for a first answer, and after exceptions taken of course, two or three month for a second - then amendments made to the bill, with more such delays, and more succeeding answers - then a cross bill filed on the other side, and a second such cause thus mounted on the shoulders of the first - then volumes heaped upon volumes of depositions - then after years thus employed, a decree obtained, by which nothing is decided-then the whole matter, and everything that has been made to grow out of it, sent to be investigated in the hermetically sealed closet of a sort of under judge called a Master - with days of attendance seperated from each other by days or weeks - length of attendance each day nominaly an hour really half or a quarter of the time. “- Unter der Bezeichnung Bill wurde ein Band notorischer Lügen vorgelegt, nach drei oder vier Monaten für eine erste Antwort und nach natürlich erhobenen Einwänden weiteren zwei oder drei Monaten für eine zweite Antwort - dann wurden Änderungen an der Klage vorgenommen mit weiteren Verzögerungen und weiteren Antworten - dann wurde eine Gegen-Bill von der Gegenseite eingereicht und eine zweite solche auf die Schultern der ersten gestellt-dann wurden Bände um Bände an Beweismitteln angehäuft - dann, nach Jahren derartiger Geschäftigkeit, erging ein Erlaß, der nichts entschied - dann wurde die ganze Angelegenheit und alles, was daraus erwachsen war, zur Untersuchung in die hermetisch verschlossene Kammer einer Art ,Unterrichters " gesandt, der Master genannt wird - mit Tagen mündlichen Verfahrens, die voneinander durch Tage oder Wochen getrennt waren - die Länge der Verhandlung nominell eine Stunde täglich, tatsächlich aber nur die Hälfte oder ein Viertel der Zeit. 
Die Lösung dieser Probleme wurde in der Verquickung der beiden Verfahren miteinander gesehen, wobei von beiden Zweigen die Vorteile beibehalten, die Nachteile hingegen eliminiert werden sollten. ${ }^{187}$ Allerdings stießen diese Vorschläge auf den erheblichen Widerstand vieler konservativer Juristen, die den Status Quo $\mathrm{zu}$ erhalten suchten. ${ }^{188}$ Daher waren statt eines großen Bruchs diverse Reformen kleinerer Art erforderlich, um die dringend notwendigen Veränderungen herbeizuführen. ${ }^{189}$

\section{a. Uniformity of Process Act 1833 und Real Property Limitation Act 1834}

Die in den dreißiger Jahren des 19. Jahrhunderts eingesetzte Reformkommission hatte das Ziel, das Common Law-Verfahren zu vereinfachen.

$\mathrm{Zu}$ diesem Zweck wurden im Uniformity of Process Act $1833^{190}$ die verschiedenen Writs für persönliche Klagen abgeschafft und ein einheitliches Writ of summons eingeführt. Allerdings wurde dieser scheinbar einschneidende Schritt dadurch entkräftet, dass der technische Name des Writs nach wie vor auch im Writ of summons aufgeführt werden musste. Dies lag daran, dass die Reformkommission aus Richtern bestand, die von der Qualität und Notwendigkeit der Pleadings überzeugt waren. ${ }^{191}$ Formulierung und Ablauf der Pleadings hingen aber vom gewählten Writ ab, so dass es weiterhin darauf ankam, im Writ of summons den richtigen Writ zu nennen. ${ }^{192}$ Ließ sich der Anspruch nicht exakt unter den Wortlaut des Writs subsumieren, der auch schon vor Einführung des Writ of summons beantragt werden musste, unterlag der Kläger nach wie vor. ${ }^{193}$

Im Real Property Limitation Act $1834^{194}$ wurden die dinglichen Klagen auf drei reduziert und 1860 zugunsten eines einheitlichen Writ of ejectment abgeschafft. ${ }^{195}$

\section{b. Common Law Procedure Acts 1852 und 1854}

Da die ersten Reformversuche nicht die von Politik und Volk erhoffte Wirkung zeigten, wurde mit den Common Law Procedure Acts 1852 und 1854 ein neuer Anlauf unternommen. Der Act von $1852^{196}$ eliminierte das Erfordernis, den technischen Namen des vormals einschlägigen Writs im Writ of summons zu zitieren. ${ }^{197}$

Die englische Rechtsprechung zeigte sich trotz der eindeutigen Aussage des Gesetzgebers sehr zurückhaltend in der Umsetzung der formalen Vereinfachung. Die faktische Abschaffung der alten Writs wurde dadurch verhindert, dass der Kläger seinen mündlichen Vortrag nach wie vor in der herkömmlichen Form der

\footnotetext{
${ }^{187}$ Jacob, S. 315 zitiert die Reformkommission mit den Worten: , The best system would seem to be one which combined the comparative brevity of the simpler forms of common law, of the principle of stating, intelligibly and not technically, the substance of the facts relied on as constituting the plaintiff's or defendant's case as distinguished from his evidence. “- Das beste System wäre eine Verbindung der vergleichsweise kurzen einfacheren Formen des Vortrags unter Common Law mit dem Prinzip [der Equity] einsichtig und untechnisch die Substanz der Tatsachen festzustellen, auf denen der Fall des Klägers oder Beklagten beruht, getrennt von seinen Beweisen.

${ }^{188}$ Sunderland, Harvard L. R. 39, S. 725, 727 f.

${ }^{189}$ Holdsworth, IX, S. 311

1902 \& 3 William IV c. 39 Uniformity of Process Act 1833

${ }^{191}$ Die Reformkommission selbst war von der Notwendigkeit der Reformen nur bedingt überzeugt. Vgl. Holdsworth, IX, S. 252.

${ }^{192}$ Holdsworth, IX, S. $251 \mathrm{f}$.

${ }^{193}$ Holdsworth, XV, S. 104

${ }^{194} 3$ \& 4 William IV c. 27 Real Property Limitation Act 1834

${ }^{195}$ Holdsworth, VII, S. 22 f.

${ }^{196} 15$ \& 16 Victoria c. 76 Common Law Procedure Act 1852

${ }^{197} 15$ \& 16 Victoria c. 76 Sec. 3 Common Law Procedure Act 1852
} 
1833 formal abgeschafften Writs halten musste. ${ }^{198}$ Spätestens in den achtziger Jahren setzte sich aber dann die Ansicht durch, dass ein Formfehler nicht mehr den gesamten Vortrag zunichte machen konnte. ${ }^{199}$ Dies war möglich, da s. 222 Common Law Procedure Act 1852 es in das Ermessen des Gerichts stellte, Formfehler in Writ oder Pleading zu verbessern oder Nachbesserungen durch die Parteien zuzulassen. ${ }^{200}$ Diese Verleihung von Ermessensspielräumen gewährte dem Richter zum Beispiel die Möglichkeit, den Parteien zu gestatten, Parteibezeichnungen im laufenden Verfahren nachzubessern ${ }^{201}$ oder im Falle des Todes des Beklagten, den Prozess gegen dessen Rechtsnachfolger weiter zu betreiben, ohne ein neues Writ of summons beantragen zu müssen. ${ }^{202}$

Schließlich führte der Common Law Procedure Act $1854^{203}$ interrogatories, wie sie im Equity-Verfahren üblich waren, auch für den Prozess nach Common Law ein. ${ }^{204}$ Darüber hinaus brachten die Common Law Procedure Acts 1852 und 1854

\footnotetext{
${ }^{198}$ Holdsworth, XV, S. 105

${ }^{199}$ Bryant v. Herbert [1878] 3 C. P. D. 390 per Bramwell L.J.: „The Common Law Commission did not abolish the forms of action in words. They recommended that; but it was supposed that, if adopted, the law would be shaken to ist foundations; so that all that could be done was to provide as far as possible that, though forms of action remained, there should never be a question what was the form. " - Die Law Commmission hat die Verwendung bestimmter Klageformen nicht abgeschafft. Sie haben dies empfohlen aber man nahm an, dass dieser Schritt das Recht bis in seine Grundfesten erschüttert hätte; alles, was getan werden konnte, um diesem Ziel dennoch möglichst nahe zukommen, war, obwohl bestimmte Klageformen weiterhin Bestand hatten, keine Klage an der Form scheitern zu lassen.

${ }^{200} 15$ \& 16 Victoria c. 76 Common Law Procedure Act $1852 \mathrm{sec} .222$ : „It shall be lawfull for the superior courts of common law, and every judge thereof, and any judge sitting at nisi prius, at all times to amend al defects and errors in any proceeding in civil causes, whether there is anything in writing to amend by or not, and whether the defect or error be that of the party to amend, or not; and all such amendments may be made with or without costs, and upon such terms as to the court or judge may seem fit; and all such amendments as may be necessary for the purpose of determining in the existing suit the real question in controversy between the parties shall be so made. " - Es soll für die Obergerichte des Common Law und jeden dort urteilenden Richter sowie jeden Richter bei Geschworenenverhandlungen rechtmäßig sein, jedweden Fehler oder Irrtum in Verfahrensfragen des Zivilrechts zu jeder Zeit zu verbessern, gleichwie, ob etwas Schriftliches zu verbessern sei und ob die Partei die Korrektur des Fehlers oder Irrtums beantragt; alle derartigen Verbesserungen sollen mit oder ohne Kostenerhebung vorgenommen werden und so geschehen, wie es das Gericht oder der Richter für richtig hält; und es sollen alle Verbesserungen durchgeführt werden, die notwendig sind, um im vorliegenden Rechtsstreit die tatsächlichen Streitfragen zwischen den Parteien festzulegen.

${ }^{201}$ Holdsworth, XV, S. $107 \mathrm{f}$.

${ }^{202}$ Day, XXIII; Holdsworth, a.a.O.

${ }^{203} 17$ \& 18 Victoria c. 125 Common Law Procedure Act 1854

${ }^{204} 17$ \& 18 Victoria c. 125 Common Law Procedure Act $1854 \mathrm{sec} .51$ : „In all Causes in any of the superior Courts, by Order of the Court or a Judge, the Plaintiff may, with the Declaration, and the Defendant may, with the plea, or either of them by Leave of the Court or a Judge may, at any other Time, deliver to the opposite Party or his Attorney [...] Interrogatories in writing upon any Matter as to which discovery may be sought, and require such Party [...] within ten Days to answer the Questions in writing by Affidavit, to be sworn and filed in the ordinary way; and any other Party or Officer omitting, without just Cause, sufficiently to answer all Questions as to which a Discovery may be sought within the above Time, or such extended Time as the Court or a Judge shall allow, shall be deemed to have commited a Contempt of the Court, and shall be liable to be proceeded against acordingly. " - In jedem Rechtsstreit vor einem Obergericht darf mit Erlaubnis des Gerichts der Kläger mit seinem ersten Klagevortrag und der Beklagte mit seinem Verteidigungsvorbringen oder beide, soweit es das Gericht gestattet, zu jeder anderen Zeit der anderen Partei oder deren Anwalt [...] schriftliche Interrogatories stellen, im Hinblick auf jede Angelegenheit, deren Klärung gewünscht werden kann, und darf verlangen, dass die Gegenpartei [...] innerhalb von zehn Tagen schriftlich und in der üblichen Weise eidesstattlich versichert darauf antwortet; und jede Partei, die es ungerechtfertigt unterlässt, alle Fragen, deren Beantwortung
} 
weitere Änderungen bei den Pleadings ${ }^{205}$ und ermöglichten den Parteien, sich darauf zu einigen, den Streit von Berufsrichtern ohne Jury entscheiden zu lassen. ${ }^{206}$

\section{c. Admiralty Act 1840}

Der Admiralty Act $1840^{207}$ hatte das Ziel, das Verfahren vor dem Court of Admiralty dem Common Law-Verfahren anzupassen. ${ }^{208}$ Dazu wurde nicht nur die $\mathrm{Zu}-$ ständigkeit erweitert, ${ }^{209}$ sondern auch eine Ermächtigung zum Erlass eigener Verfahrensvorschriften erteilt. ${ }^{210} 1859$ machte der Richter Lord Lushington von der Befugnis Gebrauch und erließ Verfahrensvorschriften. Diese erforderten zwar nicht die Verfahrenseinleitung per Writ, ähnelten aber sonst, was Verfahrenseinleitung und Pleadings betraf, stark dem Common Law-Verfahren. ${ }^{211}$

\section{d. Equity Procedure Acts 1852}

Der erste Equity Procedure Act $1852^{212}$ betraf die Organisation innerhalb der Chancery und führte, wie es in den Common Law Gerichten bereits üblich war, ein Kammersystem mit einem erkennenden Richter ein. ${ }^{213}$

Der zweite Equity Procedure Act $1852^{214}$ betraf hauptsächlich Verfahrensfragen. Das Ausstellen eines separaten Writ of subpoena wurde abgeschafft und statt dessen die Ladung des Beklagten zum Gerichtstermin auf der Bill of complaint eingetragen. ${ }^{215}$

Die Bill of complaint war von weiteren Neuerungen betroffen. Sie musste nicht mehr von Hand ausgefüllt werden, sondern durfte gedruckt werden. ${ }^{216}$ Der Tatsachenvortrag in der Bill musste so knapp wie möglich gehalten und in fortlaufend nummerierte Paragraphen gegliedert werden. ${ }^{217}$ Im übrigen musste die Bill noch das genaue Begehren des Klägers beinhalten. ${ }^{218}$ Die Interrogatories wurden hingegen nicht mehr in der Bill gestellt, sondern der anderen Partei in einem gesonderten Schriftsatz mitgeteilt. ${ }^{219}$ Im Antwortschriftsatz des Beklagten konnte er seinerseits Interrogatories formulieren, ohne eine Cross Bill ausfüllen zu müssen. ${ }^{220}$ Die Zahl der Schriftsätze wurde auf drei begrenzt. ${ }^{221}$ Letztlich bereiteten die Equity Procedure Acts 1852 den Boden für die 21 Jahre später erfolgende Verschmelzung der Gerichtszweige. ${ }^{222}$

verlangt werden kann, innerhalb der oben angegebenen oder vom Richter verlängerten Frist hinreichend zu beantworten, soll so betrachtet werden, als hätte sie sich der Mißachtung des Gerichts schuldig gemacht und soll dementsprechend haftbar sein.

${ }^{205}$ Siehe dazu Bieresborn, S. 510 ff..

${ }^{206} 17$ \& 18 Victoria c. 125 Common Law Procedure Act $1854 \mathrm{sec} .1$

${ }^{207} 3$ \& 4 Victoria c. 65 Admiralty Act 1840

${ }^{208}$ Holdsworth, XV, S. 127

${ }^{209}$ Siehe oben B. I. 4. b.

${ }^{210} 3$ \& 4 Victoria c. 65 Admiralty Act $1840 \mathrm{sec} .18$

${ }^{211}$ Ausführlich dazu Coote, S. 171-191

${ }^{212} 15$ \& 16 Victoria c. 80 Equity Procedure Act 1852

${ }^{213}$ Baker, S. 131; Holdsworth, I, $444 \mathrm{f}$.

${ }^{214} 15$ \& 16 Victoria c. 86 Equity Procedure Act 1852

21515 \& 16 Victoria c. 80 Equity Procedure Act 1852 sec. 2-5; vgl. auch Holdsworth, XV, S. 118

${ }^{216} 15$ \& 16 Victoria c. 80 Equity Procedure Act 1852 sec. 1

${ }^{217} 15$ \& 16 Victoria c. 80 Equity Procedure Act 1852 sec. 10

${ }^{218} 15$ \& 16 Victoria c. 80 Equity Procedure Act 1852 sec. 10

21915 \& 16 Victoria c. 80 Equity Procedure Act 1852 sec. 12

${ }^{220} 15$ \& 16 Victoria c. 80 Equity Procedure Act 1852 sec. 19

${ }^{221}$ Es stand im Ermessen des Gerichts, Ergänzungen zuzulassen. 15 \& 16 Victoria c. 80

Equity Procedure Act $1852 \mathrm{sec} .10$

${ }^{222}$ Holdsworth, XV, S. $121 \mathrm{ff}$. 


\section{e. Die Judicature Acts 1873-75}

Die Judicature Acts $1873-75^{223}$ stellten bis zur Einführung der Civil Procedure Rules 1998 (CPR) den größten Schnitt in das englische Zivilverfahren dar und brachten Änderungen und Vereinfachungen, die das Gesicht des Zivilprozesses in England grundlegend veränderten.

Vor allem führte man die verschiedenen Gerichtszweige zusammen und beseitigte damit die Trennung von Common Law-, Equity- und Admiralty-Gerichten. Die Gerichte wurden zusammengefasst und bildeten den High Court of Justice. Die frühere Trennung fand sich zwar weiterhin in den Abteilungen des High Court wieder, dies hatte aber ausschließlich im Rahmen der Geschäftsverteilung Bedeutung. ${ }^{224}$ Es entstanden die Queen's (bzw. King's) Bench Division, Common Pleas Division, Exchequer Division, Probate Division, Divorce Division und Admiralty Division. Jeder Richter hatte die gleichen Rechte und Pflichten, gleichgültig, in welcher Abteilung er saß. ${ }^{225}$ Von besonderer Bedeutung war dabei, dass jeder Richter sowohl Common Law- als auch Equity-Regeln anzuwenden hatte, ungeachtet, ob er in einer Abteilung urteilte, die früher ein Common Law-Gericht darstellte oder zur Chancery gehörte. Dies hatte den Effekt, dass den von den einzelnen Gerichten entwickelten Rechtsbereichen und Normenkomplexen die Bindung an die Gerichte entzogen wurde. Die Regeln zum Verhältnis von Equity zu Common Law wurden gesetzlich so fixiert, wie sie seit dem Earl of Oxford's Case Ca $^{226}$ galten: In Konfliktfällen behielt die Equity die Oberhand. ${ }^{227}$

Regelungsziel der Reform und insbesondere der Zusammenfassung der Gerichtszweige war es, das materielle Recht zu vereinheitlichen, indem man den einzelnen Gerichten die Kompetenz nahm, ,ihr eigenes Recht" fortzuentwickeln. Daneben diente die Reform der Vereinheitlichung und Beschleunigung des verfahrens. ${ }^{228}$ Die einzelnen Divisions sollten durch die interne Geschäftsverteilung einen gewissen Spezialisierungsgrad erhalten, um die Verfahren effizient und schnell zu Ende zu bringen. Zur Vermeidung von Ungerechtigkeiten hatte jede Division das gleiche Verfahren anzuwenden. ${ }^{229}$ Die meist konservativen Richter neigten jedoch dazu, dieses Reformziel zu untergraben, indem sie oft die alten Verfahrenscharakteristika beibehielten, die mit dem materiellen Recht verbunden waren, das jetzt in ihren Zuständigkeitsbereich fiel. ${ }^{230}$ Dieser Tendenz versuchte der Gesetzgeber entgegenzuwirken. So wurde in den Supreme Court of Judicature Act 1873 ein $\operatorname{Code}^{231}$ eingefügt und 1875 ergänzt und geändert ${ }^{232}$, der das Zivilverfahren in 63

\footnotetext{
${ }^{223}$ Unter diesem Sammelbegriff werden folgende Gesetze erfaßt: Supreme Court of Judicature Act 1873, 36 \& 37 Victoria c. 66; Supreme Court of Judicature Act (Commencement Act) 1874, 37 \& 38 Victoria c. 83; Supreme Court of Judicature Act (1873) (Amendment Act) 1875, 38 \& 39 Victoria c. 77.

${ }^{224}$ Jacob, S. 304; Vollkommer, ZZP 73 (1960), S. 145, 152 f.

${ }^{225}$ Vollkommer, a.a.O. S. 153

${ }^{226}$ S.o. Fn. 125

${ }^{227}$ Supreme Court of Judicature Act 36 \& 37 Victoria c. 66 s. 25 (11): Generally in all matters not hereinbefore particularly mentioned, in which there is any conflict or variance between the rules of equity and the rules of the common law with reference to the same matter, the rules of equity shall prevail. - Im Allgemeinen soll in allen nicht gesondert geregelten Angelegenheiten, in denen ein Konflikt oder Unterschied zwischen den Regeln der Equity und denen des Common Law hinsichtlich der gleichen Angelegenheit besteht, die Equity den Vorrang haben.

${ }^{228}$ Jacob, S. 307

${ }^{229}$ Jacob, S. 308

${ }^{230}$ Jacob, S. 308

${ }^{231}$ Supreme Court of Judicature Act 36 \& 37 Victoria c. 66 s. 69

${ }^{232}$ Supreme Court of Judicature Act (1873) (Amendment Act) 1875, 38 \& 39 Victoria c. 77 ss.16 und 33
} 
Orders regelte. Die einzelnen Änderungen, die hier von Interesse sind, werden im Folgenden kurz dargestellt.

\section{aa. Klagearten}

Die Klagen wurden mittels eines Writ of summons eingeleitet und hießen ohne weitere Differenzierung Actions. Der technische Name des Writ war nicht zu nennen, jedoch war der Kläger verpflichtet, auf dem Writ den Inhalt seines Anspruches möglichst genau zu bezeichnen. ${ }^{233}$ Eine Unterscheidung zwischen den Writs und der Bill of complaint war gerade nicht mehr vorzunehmen, da die Gerichte at law, wie in equity entscheiden mussten ${ }^{234}$. Wenn der Kläger annahm, der Beklagte habe keine Verteidigungsmöglichkeiten, konnte er seinen Vortrag beschwören, worauf der Beklagte aufgefordert wurde, sich innerhalb einer bestimmten Frist zu verteidigen. Tat er dies nicht, erging ex parte ein Urteil zugunsten des Klägers. ${ }^{235}$ Die Writs wurden nicht mehr in der Chancery ausgestellt, sondern in District Registries, die über das gesamte Land verteilt waren. ${ }^{236}$

\section{bb. Objektive Klagehäufung}

Mit der Zusammenlegung der Gerichte wurde die objektive und subjektive Klagehäufung grundsätzlich möglich. Bis dahin konnte vor der Chancery immer nur ein Anspruch geltend gemacht werden, da das Equity-Verfahren durch die Bill of complaint eingeleitet wurde, die nur eine bestimmte Beschwerde enthalten konnte. ${ }^{237}$ Im Common Law-Verfahren war bis dahin die objektive Klagehäufung möglich, eine subjektive Klagehäufung dagegen ausgeschlossen. ${ }^{238}$

\section{cc. Statements of claim}

Zum ersten Vorbringen des Klägers wurden einheitlich die Statements of claim, in denen er nach einem kurzen Tatsachenvortrag sein Klageziel darlegte, wie es bis dahin nur im Equity-Verfahren üblich gewesen war. ${ }^{239}$ Wie bei allen weiteren Pleadings waren die Parteien zu Knappheit und Präzision verpflichtet. ${ }^{240}$

\section{dd. Interrogatories}

Den Parteien wurde die Möglichkeit gegeben, Interrogatories zu formulieren, wie sie aus dem Equity-Verfahren bekannt waren, um die Beweisbedürftigkeit bestimmter Tatsachen festzustellen. ${ }^{241}$ Von dieser Möglichkeit machten Common Law-Praktiker nur sehr zögerlich Gebrauch, da das System dem Common LawPrinzip widersprach, jede Partei habe ihre eigene Sachverhaltssicht darzustellen und zu substantiieren, ohne Fakten von der Gegenseite zu erfahren. ${ }^{242}$

\section{f. Zusammenfassung}

Die Prozessrechtsreformen des 19. Jahrhunderts veränderten den englischen Zivilprozess grundlegend. Dies geschah durch das Zusammenlegen der Gerichtszweige

\footnotetext{
${ }^{233}$ Supreme Court of Judicature Act 36 \& 37 Victoria c. 66 Order III

${ }^{234}$ S. o. B.II.1.e.

${ }^{235}$ Supreme Court of Judicature Act 36 \& 37 Victoria c. 66 Order III; Holdsworth, XV, S. 130 f.

${ }^{236}$ Jacob, S. 305

${ }^{237}$ Holdsworth, XV, S. 133

${ }^{238}$ Holdsworth, XV, S. 133

${ }^{239}$ Baker, S. 108

${ }^{240}$ Supreme Court of Judicature Act 36 \& 37 Victoria c. 66 Order XVIII

${ }^{241}$ Bieresborn, S. 521

${ }^{242}$ Jacob, S. 316
} 
und durch neue Verfahrensvorschriften, die bezweckten, die Vorzüge der verschiedenen Prozessformen zu vereinen und dabei die bestehenden Nachteile zu eliminieren.

Das Writ-Verfahren, welches das englische Recht bis dahin geprägt hatte, wurde praktisch abgeschafft, indem man die materiellen Ansprüche des Klägers vom Rechtsbehelf und der prozessualen Einleitungsform löste. Damit verlor das englische Zivilrecht zumindest äußerlich seinen aktionenrechtlichen Charakter. Statt dessen ging man zur Verfahrenseinleitung mittels eines einheitlichen Writ of summons über, wie er aus dem Verfahren in equity bekannt war. Es kam nicht mehr auf technische Klagebezeichnungen an, sondern auf die Frage, ob der vorgetragene Sachverhalt einen materiellen Anspruch hergab. Diese materiellen Ansprüche waren ursprünglich aus den Writs entstanden. Äußerlich hatte sich das englische Recht aber von den Rechtsbehelfen zu materiellen Ansprüchen gewandelt.

Aus dem Common Law-Verfahren wurde die Knappheit und Präzision der Vorträge übernommen, um der Weitschweifigkeit, die im Equity-Verfahren geherrscht hatte $^{243}$, vorzubeugen. Dies galt für den ersten Klägervortrag, wie für die gesamten Pleadings. Das Formulieren von Interrogatories wurde erlaubt, jedoch selten praktiziert. Im Allgemeinen wurden die Formzwänge stark gelockert, was sich zum Beispiel an der Einführung der objektiven Klagehäufung zeigte.

Die Reformen des 19. Jahrhunderts passten somit den Zivilprozess und die Gerichtsverfassung den veränderten Bedürfnissen einer Gesellschaft an, die durch die Industrialisierung ein neues Gesicht bekommen hatte.

\section{Der einleitende Schriftsatz im englischen Zivilprozess von den Reformen des 19. Jahrhunderts bis zur Einführung der Civil Procedure Rules 1998}

Bis zur Einführung der CPR 1998 stellte sich der englische Zivilprozess im Wesentlichen so dar, wie er durch die Reformen des 19. Jahrhunderts geschaffen worden war. Es wurden jedoch auch im Laufe des 20. Jahrhunderts laufend Änderungen vorgenommen, deren umfangreichste der Supreme Court Act $1981^{244}$ war. ${ }^{245}$

Prägendes Merkmal des Prozessrechts war, dass unterschiedliche Verfahrensvorschriften für die Untergerichte (County Court Rules, CCR) und die Obergerichte (Rules of Supreme Court, RSC) galten. ${ }^{246}$ Bei beiden Rules handelte es sich nicht um parlamentarische Rechtsakte. Sowohl CCR als auch RSC wurden vom Rule Comittee des Supreme Court erlassen. ${ }^{247}$

Das Verfahren nach beiden Rules bestand aus einem schriftlichen Vorverfahren (Pre-trial procedure oder Interlocutory proceedings) vor einem Master oder Registrar $^{248}$ und einem mündlichen Hauptverfahren (Trial) vor einem Einzelrichter (Justice). ${ }^{249}$ In der Regel waren die einzelnen Verfahrensabschnitte voneinander getrennt. Grundsätzlich war das Ergebnis des Vorverfahrens für das Hauptverfahren bindend und konnte selbständig per Appeal ${ }^{250}$ angefochten werden.

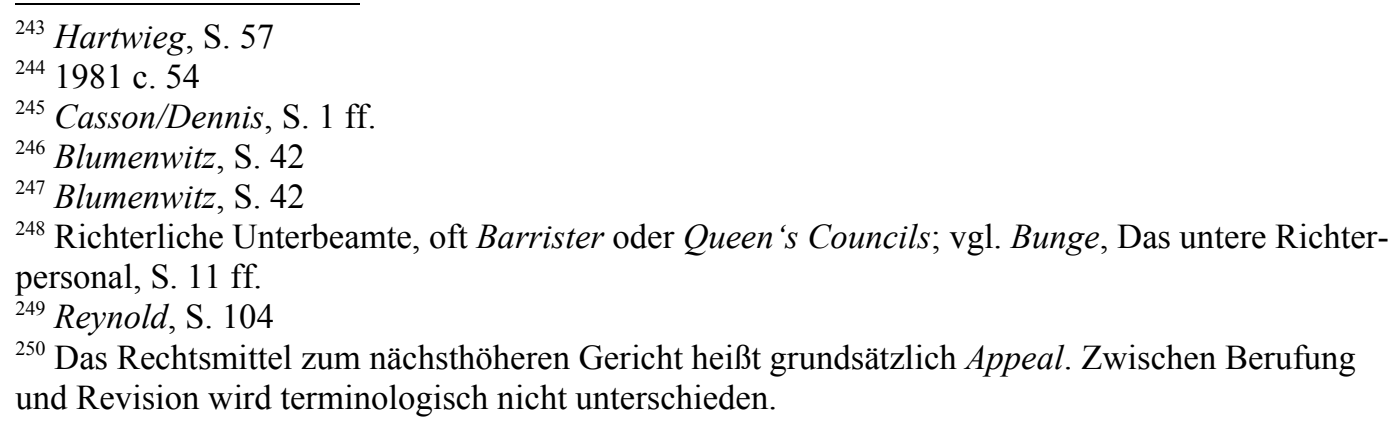




\section{a. Das Verfahren vor dem High Court nach den RSC}

Das Verfahren vor dem High Court of Justice richtete sich nach den RSC und unterlag damit dem komplexeren Regime. Auf die relevanten Unterschiede im Verfahren nach den CCR wird im Anschluss an die Darstellung des RSC-Verfahrens eingegangen.

\section{aa. Ausstellung des Writ of summons (Issue of the writ of summons)}

Das Verfahren vor dem High Court wurde einheitlich vor allen Abteilungen mit dem Writ of summons eingeleitet, RSC O. 5 r. 1-4. ${ }^{251}$ Bei der Erstellung des Writ of summons füllte der Kläger oder dessen Rechtsbeistand ein bei Gericht erhältliches Formular in dreifacher Ausfertigung aus. Ein Exemplar war für den Beklagten bestimmt, ein weiteres Exemplar verblieb bei Gericht. ${ }^{252}$

Das Writ enthielt zwar noch das königliche Wappen, der Ladungsbefehl durch den Monarchen wurde jedoch mit dem Supreme Court Act 1981 abgeschafft. ${ }^{253}$

Auf der Frontseite des Formulars musste der Kläger die zuständige Abteilung des High Courts eintragen ${ }^{254}$ sowie ggf. die Außenstelle, bei der das Writ beantragt worden war, wenn dies nicht am High Court in London selbst geschehen war. Des weiteren musste der Kläger seinen Namen und seine Adresse sowie Namen und Adresse des bzw. der Beklagten eintragen und bei mehreren Beklagten diese durchnummerieren. Nähere Beschreibungen der Parteien waren in aller Regel nicht erforderlich. Zusätze mussten gemacht werden, wenn sonst Verwechslungen eintreten konnten oder wenn sich das Geschlecht nicht eindeutig aus dem Namen ergab. Ebenso war eine etwaige Minderjährigkeit (mit dem Attribut Minor) oder Unzurechnungsfähigkeit (mit dem Attribut Patient) zu vermerken, da dadurch die Prozessfähigkeit der Partei berührt wurde. Oft konnte dies erst nach Zustellung erfolgen, da diese Fakten dem Kläger unter Umständen nicht bekannt waren. Bei prozessunfähigen Parteien wurde ein Vertreter (Klägervertreter: Next friend; Beklagtenvertreter: Guardian in litem) auf dem Writ vermerkt. ${ }^{255}$ Hatte der Kläger einen Prozessbevollmächtigten, war dessen Name und Adresse auf dem Writ zu vermerken.

Neben dem Ausstellungsdatum und dem Aktenzeichen enthielt die Writ-Vorderseite noch eine Beklagtenbelehrung, wonach er innerhalb von 14 Tagen den klägerischen Anspruch zu befriedigen oder eine Empfangserklärung an das Gericht zu senden hatte. Nichtbefolgung dieser Anordnung konnte ein Urteil zugunsten des Klägers ohne weitere Anhörung des Beklagten nach sich ziehen. ${ }^{256}$

\footnotetext{
${ }^{251} \mathrm{Zu}$ den Ausnahmen nach RSC O. 5 r. 4 siehe unten B.II.2.a.gg.

${ }^{252}$ Langan/Henderson, S. 40

${ }^{253}$ Casson/Dennis, S. 17

${ }^{254}$ Fehler können nach Brady v. Barrow Steelworks Ltd. [1965] 2 Q.B. 182 jederzeit nachgebessert werden.

${ }^{255}$ Langan/Henderson, S. 31

${ }^{256}$ Musterexemplare bei Barnard/Houghton, S. 15 und 78: Within 14 days after the service of this writ on you, counting the day of service, you must either satisfy the claim or return to the court office mentioned below the accompanying Acknowledgement of Service stating therein wether you intend to contest these proceedings.

If you fail to satisfy the claim or return the Acknowledgement within the time stated, or if you return the Acknowledgement without stating therein an intention to contest the proceedings, the Plaintiff may proceed with the action and judgement may be entered against you forthwith without further notice. - Innerhalb von 14 Tagen nach Zustellung dieses writs, den Tag der Zustellung mitgezählt, müssen Sie entweder den Anspruch befriedigen oder die beiliegende Empfangsbestätigung an die unten aufgeführte Geschäftsstelle des Gerichts zurücksenden und darin vermerken, ob Sie beabsichtigen, das Verfahren aufzunehmen.
} 
Auf der Rückseite des Writs wurde das Indorsement of claim eingetragen. Dabei musste der Kläger knapp die Natur seines Anspruches darlegen und seinen Antrag bezeichnen (General Indorsement), RSC O. 6 r. 2. Das Indorsement of claim diente dazu, den Beklagten zu informieren, aus welchem Lebenssachverhalt und welchem rechtlichen Bereich er verklagt wurde. Dafür war es ausreichend, den Anspruch grob zu umreißen, ohne auf alle Tatsachenbehauptungen einzugehen oder bei Geldforderungen die Summe zu nennen. ${ }^{257}$ Einerseits entfaltete das Writ auch ohne Angabe von Gründen Druck beim Beklagten. ${ }^{258}$ Andererseits sollte dem Beklagten möglichst wenig ,verraten“ werden, ${ }^{259}$ weil der Kläger nicht riskieren wollte, sich in einem frühen Stadium auf einen bestimmten Sachvortrag festzulegen. ${ }^{260}$ Es ist vielmehr typische Eigenart des anglo-amerikanischen Zivilprozesses, es der Gegenseite zu überlassen, Offenlegung der Tatsachen (Discovery) $\mathrm{zu}$ beantragen.

Das General Indorsement war nicht bereits der erste klägerische Sachvortrag. Dieser erfolgte in der Regel als Statement of claim in einem gesonderten Schriftsatz. ${ }^{261}$ Allerdings stand es dem Kläger frei, statt des General Indorsement auf der Rückseite des Writs das Statement of claim (Special Indorsement) einzutragen. Da auf der Rückseite des Writs nur wenig Platz war, bot sich dieses Vorgehen nur in einfach gelagerten Fällen an, da sonst aufgrund des Umfanges der Darstellung ohnehin ein gesonderter Schriftsatz eingereicht werden musste. Erwartete der Kläger keine Verteidigung des Beklagten, war es sinnvoll, das Statement of claim auf die Writ-Rückseite zu schreiben, da dann ohne gesonderte Zustellung des Statement of claim ein Säumnisurteil zugunsten des Klägers ergehen konnte. ${ }^{262}$

Auf der Rückseite des Writs war weiterhin zu vermerken, ob der Kläger aus eigenem Recht klagte, oder als Rechtsnachfolger bzw. gesetzlicher Vertreter, RSC O. 6 r 3 (1). War der Klagegrund (Cause of action) nicht in dem Bezirk entstanden, in dem das Writ ausgestellt wurde, musste auch dies auf der Rückseite vermerkt werden, RSC O. 6 r. 4. Schließlich war dem Writ das Acknowledgement of claim-Formular beizulegen, RSC O. 6 r. 7 (5).

Das Writ wurde mit Siegelung durch einen Gerichtsbeamten wirksam. Vorher musste die fällige Gebühr bezahlt werden. ${ }^{263}$

Der Zeitpunkt der Wirksamkeit entschied über die Frage, ob die Verjährungsfrist abgelaufen war. ${ }^{264}$ Stellte der Kläger das Writ dem Beklagten nicht innerhalb der gesetzlichen Frist von vier Monaten zu, entfiel die Verjährungsunterbrechung. ${ }^{265}$ Zwei Punkte sind besonders zu beachten:

\footnotetext{
Wenn Sie versäumen, den Anspruch zu befriedigen oder die Empfangsbestätigung innerhalb der angegebenen Frist zurückzusenden, oder die Empfangsbestätigung zurücksenden, aber nicht angeben, ob Sie das Verfahren aufnehmen wollen, dann kann der Kläger seine Klage weiter verfolgen und ein Urteil kann gegen Sie ergehen, ohne dass eine weitere Benachrichtigung erfolgt. 
1. Bei der Ausstellung des Writs handelte es sich um reine Parteihandlung. ${ }^{266}$ Bis zur mündlichen Verhandlung erfolgte in der Regel ${ }^{267}$ keine inhaltliche Prüfung des Writs durch das Gericht. ${ }^{268}$

2. Beim Writ of summons handelte es sich nicht um die Klage mit Klagebegründung, ${ }^{269}$ sondern nur um den Ladungsbefehl an den Beklagten.

\section{bb. Zustellung des Writ of summons (Service of the writ of summons)}

Nach Ausstellung des Writs musste der Kläger dem Beklagten das Writ zustellen. Hatte der Beklagte einen Anwalt, wurde gewöhnlich dem Anwalt das gesiegelte Writ-Original mit einer Kopie für den Anwalt zugesandt. Der Anwalt musste das Original mit der Annahmebestätigung zurückzuschicken, RSC O. 10 r. 1 (4). Hatte der Beklagte keinen Anwalt beauftragt, wurde das Writ an seine letzte bekannte Adresse geschickt, RSC O. 10. Die Zusendung erfolgte gewöhnlich durch First Class Post. ${ }^{270}$ Solange das Gegenteil nicht bewiesen war, galt eine Zustellungsvermutung für den siebten Tag nach Absendung, RSC O. 10 r. 1 (3) (a). ${ }^{271}$ In Ausnahmefällen kam es auch zu einer persönlichen Zustellung durch Übergabe des Writs im verschlossenen Umschlag an den Beklagten, RSC O. 65 r. 2-7. Auch die persönliche Zustellung oblag in der Regel dem Kläger, nicht dem Gericht, wobei er sich einer Zustellungsfirma bedienen durfte. Anderes galt bei Zustellung einer Ladung eines ausländischen Gerichts, welche im Rechtshilfeverfahren von englischen Gerichtsbeamten zugestellt wurde. ${ }^{272}$ Nicht möglich war die Zustellung des Writs per Fax. ${ }^{273}$ Nur Schriftstücke, die nicht persönlich zugestellt werden mussten oder keinen verfahrenseinleitenden Charakter hatten, durften per Fax zugestellt werden, RSC O. 65 r. 5 (2B). Stellte der Kläger das Writ nicht innerhalb von vier ${ }^{274}$ Monaten $\mathrm{zu}^{275}$, verfiel es, es sei denn, dem Kläger wurde auf Antrag eine Verlängerung des Writs gewährt, wenn er sachliche Gründe für die Nichtzustellung anführen und glaubhaft machen konnte. ${ }^{276}$ Dann war auch eine Verlängerung nach Fristablauf noch möglich. Dieses sogenannte renewal of the writ wurde zum Beispiel gewährt, wenn das Writ nicht zugestellt werden konnte, weil sich der Beklagte im Ausland aufhielt. Die Verlängerung wurde jedoch in der Regel nicht genehmigt, wenn der Anspruch zwischenzeitlich verjährt war. ${ }^{277}$

\footnotetext{
${ }^{266}$ K. Schmidt, S. $108 \mathrm{f}$.

${ }^{267}$ Gesetzliche Ausnahmen gab es z.B. für Grundstücksangelegenheiten (Leasehold Property (Repairs) Act 1938 s.1), unzurechnungsfähigen Personen (Mental Health Act 1959 s. 141). Lords, dass das Zustellungsdatum nicht der siebte Tag ist, wenn eine der Parteien darlegen kann, dass der Beklagte früher oder später Kenntnis von dem Writ hatte. In diesem Fall wurde der Beklagte darüber informiert, dass ein Writ in seinem Briefkasten lag, worauf er es unterließ nach Hause zu fahren, sondern sich in einem Hotel einmietete und das Land am nächsten Tag wieder verließ. Das House of Lords entschied, aus dem Verhalten des Beklagten ließe sich schließen, dass er Kenntnis von dem Writ hatte, obwohl der Umschlag nicht geöffnet wurde und nie in die Hände des Beklagten gelangte.

${ }^{272}$ Jacob, S. 174

${ }^{273}$ Hastie \& Jenkerson v. McMahon [1991] All ER 255

${ }^{274}$ Bis 1992 betrug die Frist zwölf Monate. Vgl. auch K. Schmidt, S. 110.

${ }^{275}$ Siehe zur Berechnung der Frist Trow v. Ind Coope (West Midlands) [1967] 2 Q.B.567

${ }^{276}$ K. Schmidt, S. 110 f. mit Beispielen.

${ }^{277}$ Heaven v. Road \& Rail Wagons Ltd. [1965] 2 Q.B. 355: Writ wurde während der Vergleichsverhandlungen beantragt, Verlängerung des Writs wurde aber erst über ein Jahr nach Scheitern der
} 
War der Beklagte nicht anwesend oder verweigerte er die Annahme, konnte das Writ bei der persönlichen Zustellung auch einer anderen Person in der Umgebung des Beklagten übergeben werden, vorausgesetzt, diese Person wurde über den Inhalt des Umschlages aufgeklärt. ${ }^{278}$ War der Beklagte nicht auffindbar und die Zustellung infolge dessen erfolglos, konnte auf Anordnung des Gerichts eine Ersatzzustellung durch öffentliche Bekanntmachung erfolgen. ${ }^{279}$

Die Zustellung ins Ausland (Service out of the jurisdiction) richtete sich ab der Ratifizierung des EuGVÜ 1983 durch das Vereinigte Königreich nach dem neu gefassten RSC O. 11 r. 1, wonach zwischen Zustellung in Fällen, in denen das EuGVÜ anwendbar war, und solchen, wo es nicht zur Anwendung kam, unterschieden werden musste. Ergab sich die Zuständigkeit des englischen Gerichts aus dem EuGVÜ, konnte das Writ ohne Genehmigung des Gerichts im Ausland zugestellt werden, RSC O. 11 r. 1 (2) (a) ${ }^{280}$, vorausgesetzt das Verfahren war nicht schon in einem anderen Vertragsstaat anhängig, Art. 21 EuGVÜ. Die Frist zur Absendung der Empfangsbestätigung betrug dabei 21 Tage, RSC O. 11.r. 1(3) (a). War eine Zustellung ins Ausland nach RSC O. 11.r. 1 (2) (a) nicht möglich, durfte das Writ einem Beklagten, der seinen Wohnsitz in einem Vertragsstaat hatte, auch dann nicht zugestellt werden, wenn er sich vorübergehend in England aufhielt. ${ }^{281}$

Der entscheidende Zeitpunkt für den Ablauf der Verjährungsfrist war zwar die Ausstellung des Writs. Für die Frage, wann die Klage im Sinne des Art. 21 EuGVÜ anhängig gemacht wurde, kam es aber auf die Zustellung an. ${ }^{282}$

Zustellungen ins Ausland, bei denen das EuGVÜ keine Anwendung fand, richteten sich nach RSC O. 11 r. 1 (1), der die Rechtslage festschrieb, wie sie vor Einführung des EuGVÜ für alle Auslandszustellung gegolten hatte,. Zustellungen waren nur mit vorheriger Genehmigung des Gerichts, deren Erteilung in dessen Ermessen stand, zulässig. Dabei kam eine Genehmigung nur unter engen Voraussetzungen in Frage. ${ }^{283}$ Allerdings konnte einem Beklagten, der sich vorübergehend im Inland aufhielt, das Writ persönlich zugestellt werden, solange die Zustellung nicht schikanös (oppressive or vexatious) ${ }^{284}$ oder rechtsmissbräuchlich war. ${ }^{285}$

Verhandlungen beantragt, als der Anspruch bereits verjährt war. Verlängerung wurde nicht genehmigt. Anders in Battersby v. Anglo-American Oil Co Ltd. [1945] K.B. 23.

${ }^{278}$ Banque et Francaise v. Clarke [1894] W.N. 203

${ }^{279}$ Afro Continental Nigeria v. Meridian Shipping Co S.A. (The Vrontados) [1982] Lloyd's Rep. 241

${ }^{280}$ Nach O. 11 r. 1 (2) (b) galt das gleiche, wenn der High Court aufgrund eines anderen Gesetzes zuständig war.

${ }^{281}$ Casson/Dennis, S. 22; Langan/Henderson, S. 50

${ }^{282}$ Dresser (UK) Ltd v. Falcongate [1992] All ER 450 (C.A.); Neste Chemicals SA v. DK Line SA [1994] 3 All E R 180 (C.A.)

${ }^{283}$ Die wichtigsten Tatbestände des RSC O. 11 r. 1 (1) waren unter anderem Klagen, die in Verbindung mit Grundbesitz im Inland standen (O. 11 r. 1 (1) (g-i)), Klagen in Verbindung mit Verträgen, die im Inland geschlossen wurden oder dort erfüllt werden sollten oder bei denen englisches Recht das anwendbare Recht war (O. 11 r. 1. (1) (d-e)) sowie Klagen aus Delikt, bei denen die deliktische Handlung im Inland vorgenommen wurde oder der Schaden dort entstanden war (O. 11 r. 1 (1) (f)).

${ }^{284}$ Colt v. Sarlie [1966] 1 W.L.R. 440: Die Zustellung ist nur in Ausnahmefällen schikanös, beispielsweise, wenn der Beklagte nach England gelockt wird, damit ihm das Writ zugestellt werden kann.

${ }^{285}$ In Baroda v. Wildenstein [1972] 2 Q.B. 283 wurden die Voraussetzungen für eine Einstellung des Verfahrens aufgrund schikanöser oder rechtsmißbräuchlicher Zustellung noch dahingehend verschärft, dass der Beklagte auch beweisen mußte, dass eine Einstellung keine Ungerechtigkeit für den Kläger darstelle. 


\section{cc. Acknowledgement of Service}

Durch das Zurücksenden der Empfangsbestätigung (Acknowledgement of service) an das Gericht zeigte der Beklagte seine Bereitschaft an, den Rechtsstreit aufzunehmen. ${ }^{286}$ Dies musste der Beklagte auf dem Formularblatt ankreuzen. ${ }^{287}$ Weiterhin musste der Beklagte seine genaue Anschrift beziehungsweise die seines Anwaltes angeben ${ }^{288}$. Die Abgabe der Empfangserklärung konnte auch unter Protest gegen die Ausstellung oder Zustellung des Writs erfolgen, RSC O. 12 r. 7.

\section{dd. Statement of claim und sich daran anschließende Pleadings}

Das Statement of claim war der erste Schriftsatz des Klägers. Er folgte auf das Acknowledgement of service und eröffnete die Pleadings der Parteien, es sei denn, der Kläger hatte sein Statement of claim auf der Rückseite des Writs eingetragen. ${ }^{289}$ Unter Pleadings verstand man den Austausch von Schriftsätzen zwischen den Parteien im Vorverfahren, durch die alle Streitpunkte für die mündliche Hauptverhandlung festgelegt werden sollten. Diese Schriftsätze wurden in der Hauptverhandlung vom Gericht nicht zur Urteilsfindung herangezogen. Das Gericht urteilte ausschließlich aufgrund des mündlichen Parteivorbringens in der Hauptverhandlung, ohne dass eine Einbeziehung des schriftlichen Parteivortrags aus dem Vorverfahren durch Bezugnahme auf die Pleadings möglich gewesen wäre. ${ }^{290}$ Die Pleadings hatten den Zweck, die Gegenpartei über die Tatsachen zu informieren, die im Hauptverfahren verwandt werden sollten ${ }^{291}$ und vor allem die streitigen Punkte, Anträge und den Tatbestand zu identifizieren und festzulegen. ${ }^{292}$ Keine der Parteien durfte im Hauptverfahren Tatsachen darlegen, die nicht im Pleading angekündigt worden waren, ${ }^{293}$ oder Beweise anbieten, deren Thema über die in den Pleadings angeführten Behauptungen hinausging. ${ }^{294}$

Das erste Pleading war das Statement of claim des Klägers, welches die Klagebegründung darstellte. ${ }^{295}$ Darin mussten alle Tatsachen enthalten sein, um den Anspruch schlüssig darzulegen ${ }^{296}$, sowie ein bestimmter Antrag, RSC O. 18 r. $15 .^{297}$

Auf das Statement of claim antwortete der Beklagte mit der Defence, RSC O. 18 r. 2, gegen die der Kläger wiederum per Reply, RSC O. 18 r. 3 (4), vorgehen konnte. Dies war aber nur nötig, wenn der Kläger sich nicht auf das Bestreiten des Beklagtenvortrages beschränkte, sondern seinerseits neue Tatsachen vortrug, RSC O 18 r. 14 (1). Am Ende der Pleadings ${ }^{298}$ mussten sich die Parteien auf die Streitpunkte geeinigt haben, sogenanntes Joinder of issue. Dies geschah durch ausdrückliche Erklärung, RSC O. 18 r. 14 (4), oder per Fiktion, d.h. wenn sich auf die Defence oder die Reply die andere Seite nicht mehr äußerte, wurde

\footnotetext{
${ }^{286}$ Bunge, Zivilprozess, $§ 42$

${ }^{287}$ Musterbeispiel bei Langan/Henderson, S. 411

${ }^{288}$ Ebenda.

${ }^{289}$ Siehe zu diesem eher seltenen Fall oben B.II.2.a.aa.

${ }^{290}$ Bücker, S. 14 m.w.N.

${ }^{291}$ Cohn, ZZP 73 (1960), S. 324, 332

${ }^{292}$ Vgl. zur funktionalen Ähnlichkeit mit der deutschen Richterrelationstechnik: K. Schmidt, S. 121

${ }^{293}$ Cohn, ZZP 73 (1960), S. 324, 326; Löwenkamp, S. 42 f.

${ }^{294}$ Davie v. New Merton Board Mills Ltd. [1965] 1 All ER 379

${ }^{295}$ Hartwieg, S. 62

${ }^{296}$ Bruce v. Odhams Press Ltd. [1936] All ER 294

${ }^{297}$ Langan/Henderson, S. 78

${ }^{298}$ In der Regel ist das nach der Defence oder der Reply, obwohl in Ausnahmefällen theoretisch Rejoinder und Surrejoinder sowie Rebutter und Surrebutter folgen können. Langang/Henderson, S. 87
} 
vermutet, das der gesamte Inhalt des letzten vorgebrachten Pleadings Streitpunkt sein sollte, RSC O. 18 r. 14.

\section{ee. Inhaltliche Anforderungen an die Pleadings}

Da die Pleadings schon formale Prozesshandlungen darstellten, unterlagen sie genauen inhaltlichen Anforderungen.

Insbesondere waren die Pleadings auf Tatsachenbehauptungen zu beschränken, RSC O. 18 r. 7 (1). Rechtsbehauptungen waren grundsätzlich unzulässig. Im Rahmen des Tatsachenvortrages konnten allerdings die Rechtsinstitute, die die vortragende Seite für einschlägig hielt, genannt werden, sog. raising a point of law, RSC O. 18 r. 11. Die einzelnen Tatbestandsmerkmale hingegen durften nicht aufgeführt werden. ${ }^{299}$

Aus dem Grundsatz des reinen Tatsachenvortrages ${ }^{300}$ folgte auch, dass das Gericht nicht an Rechtsansichten der Parteien gebunden war.

Des weiteren hatten sich die Pleadings auf die grundlegenden Tatsachen $\mathrm{zu}$ beschränken. ${ }^{301}$ Insbesondere durfte nicht schon Beweis angetreten werden. Die Beweisführung war ausschließlich Gegenstand des Trials, das Vorverfahren diente nur der Festlegung des Streitpunktes. Die vorgetragenen Tatsachen mussten grundlegend sein, d.h. es durfte nicht ausgeschlossen sein, dass sie für den Rechtsstreit von unmittelbarer Bedeutung sein konnten. ${ }^{302}$ Allerdings musste sich der Vortrag nicht auf die Fakten beschränken, die die jeweilige Partei zu beweisen hatte, um den Rechtsstreit zu gewinnen. ${ }^{303}$ Nicht vorgetragen wurden Tatsachen, für die eine rechtliche Vermutung bestand oder für die die Gegenpartei beweispflichtig war, RSC O.18 r.7(3). Welche Tatsachen als wesentlich erachtet werden mussten, richtete sich demnach sowohl nach der materiellen Rechtslage, als auch danach, ob der Verfahrensstand im Prozess den Vortrag einer Tatsache erforderlich machte. ${ }^{304}$

Der Vortrag im Pleading musste bestimmt sein und die Tatsachenbehauptungen durften sich nur widersprechen, wenn sie in ein Eventualverhältnis gestellt wurden, RSC O. 18 r. 10. Dies war von besonderer Bedeutung, da die Parteien an ihr Vorbringen gebunden waren und zwar insoweit, als der Vortrag im Trial nicht von dem im Vorverfahren abweichen durfte (rule of departure), RSC O. 18 r. 10. Aber auch innerhalb des Vorverfahrens konnten die Parteien nicht ohne weiteres $^{305}$ ihren Vortrag ändern. ${ }^{306}$

\footnotetext{
${ }^{299}$ Langan/Henderson, S. 91

${ }^{300}$ Ausnahmen hierzu sind des weiteren das Vorbringen von Rechtsfolgen der vorgetragenen Tatsachen, so z. B. die Höhe des Schadenersatzes; Drane v. Evangelou [1978] 1 W.L.R. 455; der Hinweis auf Rechtsfragen von grundsätzlicher Bedeutung, damit sich die Gegenseite diesbezüglich auf die Verhandlung vorbereiten kann; Independent Automatic Sales Ltd. v. Knowels \& Foster [1962] 1 W.L.R. 974. Schließlich müssen auch Rechtsfolgen, die sich aus bestimmten Gesetzen ergeben gesondert vorgetragen werden, z. B. die Verjährung nach dem Limitation Act 1980; RSC O 18 r. 8 (1); vgl. auch Langan/Henderson, S. 91.

${ }^{301}$ Langan/Henderson, S. 92

${ }^{302}$ Langan/Henderson, S. 93

${ }^{303}$ Langan/Henderson, S. 93

${ }^{304}$ Cohn, ZZP 73 (1960), S. 324, 328

${ }^{305} \mathrm{Zu}$ den Ausnahmen siehe RSC O. 20.

${ }^{306}$ So darf bspw. das Statement of claim nicht vom General Indorsement auf dem Writ abweichen. Vgl. auch Löwenkamp, S. 90.
} 


\section{ff. Mündlicher Vortermin und Trial}

Direkt nach dem Vorverfahren fand der Austausch relevanter Dokumente (Discovery of documents) statt, RSC O. $24^{307}$, an den sich der mündliche Vortermin anschloss, RSC O. 25. In diesem Hearing of the summons for directions ${ }^{308}$ wurden der Streitstand festgestellt und Verfahrens- und Vorbereitungsfragen geklärt. ${ }^{309}$ So mussten bei diesem nicht öffentlichen Hearing alle prozessualen Einreden vorgebracht werden, ${ }^{310}$ insbesondere Fragen der Zuständigkeit (Jurisdiction) ${ }^{311}$, Rechtshängigkeit (Lis alibi pendens) ${ }^{312}$ und Rechtskraft (Res judicata) ${ }^{313}$.

Wenn das Verfahren nicht im summarischen Verfahren ${ }^{314}$ beendet wurde, ${ }^{315} \mathrm{kam}$ es zum mündlichen Hauptverfahren (Trial).

Das Trial war die Verhandlung vor dem Richter und gegebenenfalls einer Jury. Dabei wurde über die im Vorverfahren festgelegten Streitpunkte entschieden. Es galt ausschließlich das Mündlichkeitsprinzip, so dass nicht auf Schriftsätze Bezug genommen werden konnte. ${ }^{316}$ Richter und, soweit vorhanden, die Jury gingen grundsätzlich unvorbereitet in die Verhandlung, damit gewährleistet war, dass das Urteil ausschließlich auf der mündlichen Verhandlung beruhte. ${ }^{317}$

\section{gg. Verfahrenseinleitung ohne Writ}

Es war auch möglich, ein Verfahren ohne Writ mittels Originating summons einzuleiten, wenn der einzige oder primäre Streitpunkt erkennbar mit hoher Wahrscheinlichkeit eine Rechtsfrage war, RSC O. 5 r. 4 (a), oder wenn substantieller Streit über Tatsachen nicht zu erwarten war, RSC O. 5 r. 4 (b) ${ }^{318}$ Das Originating summons wurde wie ein Writ beantragt und zugestellt. Inhaltlich unterschied es sich aber deutlich vom Writ, da es neben Namen und Anschrift der Parteien bzw. Prozessbevollmächtigten nur eine ausreichende Angabe der Klagegründe sowie der Frage, deren Klärung der Kläger begehrte, enthalten musste, RSC O. 7 r. 3 (1). Das Verfahren mit Originating summons lief ohne Pleadings ab. Stattdessen gaben die Parteien eidesstattliche Versicherungen ab, die als Beweis fungierten, da in der Regel Tatsachen nicht streitig waren, RSC O. 28 r. 1A (1) und (4). Im Anschluss daran fand eine mündliche Anhörung vor Gericht statt (Master's appointment), RSC O. 28 r. 2 (1). Dabei wurde geklärt, ob der Fall ausreichend für die Hauptverhandlung vorbereitet war und alle Behauptungen eidesstattlich versichert waren, RSC O. 28 r. 4 (3). Im Anschluss daran wurde der Fall vor einem Richter verhandelt. ${ }^{319}$

\footnotetext{
${ }^{307}$ Jacob, CMLR 1 (1963/64), S. 294, $300 \mathrm{ff}$.

${ }^{308}$ RSC O. 25; Supreme Court Practice (White Book) 25/1/1

${ }^{309}$ Barnard/Houghton, S. 284

${ }^{310}$ Umfassend dazu Bunge, Zivilprozeß, § 45.

${ }^{311}$ Im Rahmen des EuGVÜ kommen dessen Zuständigkeitsregeln zum Tragen.

${ }^{312}$ RSC O. 18 r. 19, wonach der zweite Prozess als abuse of process (Verfahrensmißbrauch) gerügt wurde. Vgl. auch Bunge, Zivilprozeß, § 45 I 6. Seit Ratifizierung des EuGVÜ, bzw. Einführung der EuGV-VO gelten für die Rechtshängigkeit vor Gerichten anderer Vertragsstaaten Art. 21-23 EuGVÜ/27 ff. EuGV-VO.

${ }^{313}$ Bunge, ZZP 92 (1979), S. 351, 359 ff.

${ }^{314}$ Summary Judgment, RSC O. 14

${ }^{315}$ Siehe dazu Bieresborn, S. 552 f.

${ }^{316}$ Bücker, S. 64; diese wurden allenfalls herangezogen, wenn der Verdacht bestand, eine Partei habe gegen die Rule of departure verstoßen.

${ }^{317}$ Bücker, S. 64

${ }^{318}$ Barnard/Houghton, S. 326; Langan/Henderson, S. 39

${ }^{319}$ Langan/Henderson, S. 299
} 


\section{b. Das Verfahren vor den County Courts nach den CCR}

Rechtssachen, die nicht vor dem High Court of Justice, sondern vor den County Courts verhandelt wurden, ${ }^{320}$ folgten prinzipiell den gleichen Grundsätzen wie die Verfahren vor dem High Court, ${ }^{321}$ allerdings nur insoweit, als der County Court Act 1959 oder die County Court Rules 1981 keine spezielleren Regelungen trafen. Die Einleitung des Verfahrens geschah mit Hilfe eines Request of summons (auch Praecipe genannt), auf dem die Namen der Parteien und der Klagegrund, bei Klagen auf Zahlung einer Geldschuld nur der Betrag, eingetragen wurden. ${ }^{322}$ Gleichzeitig musste der Kläger die Particulars of claim ausfüllen. Diese entsprachen dem Statement of claim nach den RSC, CCR O. 6 r. 1 (1), und in einfach gelagerten Fällen konnten die Particulars auf dem Request of summons eingetragen werden, CCR O. 6 r. 1 (2). Request of summons bzw. Particulars of claim im County Court-Verfahren entsprachen funktional dem Writ of summons und Statement of claim im Verfahren vor dem High Court. Writ bzw. Request stellten jeweils die Ladung dar, Statement und Particulars waren das erste Pleading. Die Zustellung wurde jedoch in der Regel nicht vom Kläger, sondern vom Gericht vorgenommen. ${ }^{323} \mathrm{Um}$ nicht Gefahr eines Versäumnisurteils zu laufen, CCR O. 9 r. 4, musste der Beklagte innerhalb von 14 Tagen seine Verteidigungsbereitschaft anzeigen, Widerklage erheben oder anerkennen, CCR O. 9 r. 2, und seine Verteidigung (Defence) darlegen. Tat er dies, wurde eine Anhörung vor dem Registrar terminiert (sog. Pre-trial review), in der das weitere Vorgehen verfügt wurde, CCR O. 17.

Nach dem Pre-trial review folgte das Verfahren im Wesentlichen den Grundsätzen vor dem High Court. ${ }^{324}$

\section{c. Zusammenfassung}

Das Verfahren des späten 19. und 20. Jahrhunderts bis zur Einführung der CPR 1998 war primär in den RSC und CCR geregelt und zeichnete sich durch einige Besonderheiten aus. Die Einleitung des Verfahrens geschah in der Regel mit Hilfe eines Writs (Requests), das allerdings ausschließlich prozessuale Bedeutung hatte und weder formell noch inhaltlich an den materiellen Anspruch gebunden war.

Nach der Verfahrenseinleitung kam es zum schriftlichen Vorverfahren, in dem die Pleadings ausgetauscht wurden. Dabei stellte das Statement (Particulars) of claim den ersten Schriftsatz dar. In Ausnahmefällen konnte der Vortrag schon auf dem Writ eingetragen werden. Sinn des Vorverfahrens war die Festlegung der Streitpunkte. Nach Abschluss des Vorverfahrens kam es zum ausschließlich mündlichen Hauptverfahren (Trial), in dem Beweis erhoben wurde. Die strenge Zweiteilung des Verfahrens wurde allerdings durch die rule of departure etwas aufgehoben, da die Parteien auch in der Hauptverhandlung an ihre Vorträge aus dem Vorverfahren gebunden waren.

Im ganzen Verfahren übte das Gericht eine rein überwachende, nicht dagegen inquisitorische Funktion aus, so dass die Verhandlungsmaxime in vollem Rahmen zum Tragen kam. Das Verfahren, wie es Ende des 19. Jahrhunderts geschaffen

\footnotetext{
${ }^{320}$ Für die meisten Klagen bestand eine konkurrierende Zuständigkeit, die die Wahl des Gerichts dem Kläger überließ, vgl. High Court and County Courts Jurisdiction Order 1991.

${ }^{321}$ Sec. 103 County Courts Act 1959

${ }^{322}$ Langan/Henderson, S. 347

${ }^{323}$ Langan/Henderson, S. 242

${ }^{324}$ Allerdings konnte ein Summary Judgment nur bei Beträgen unter GBP 500 beantragt werden, CCR O. 9 r. 14 (2).
} 
worden war, behielt seine liberale Prägung und war im 20. Jahrhundert nur vergleichsweise geringen Änderungen ausgesetzt. 


\section{Der einleitende Schriftsatz nach den CPR 1998}

1994 trat eine Kommission mit dem Ziel an, das englische Zivilverfahren zu reformieren. ${ }^{325}$ Primär sollte der Zugang zur Justiz erleichtert, die Komplexität der Regeln verringert, die Kosten sollten gesenkt und die Terminologie modernisiert werden. ${ }^{326}$ Der Abschlussreport von $1996^{327}$ wurde im Wesentlichen umgesetzt ${ }^{328}$ und trat am 26.04.1999 als Civil Procedure Rules 1998 in Kraft. ${ }^{329}$ Als Rechtsgrundlage diente der Civil Procedure Act 1997.

Die CPR 1998 stellen für England erstmalig eine einheitliche Zivilprozessordnung dar und regeln sowohl das Verfahren vor den County Courts als auch vor dem High Court of Justice.

\section{1. Überblick über die Neuerungen}

Die CPR 1998 verändern das englische Zivilverfahren in einigen Punkten grundlegend. Insbesondere wird ein neues Verständnis von der Aufgabe der Gerichte proklamiert. Das Verfahren zeichnete sich traditionell durch seinen adversativen Charakter aus, ${ }^{330}$ d. h. die Gerichte verstanden sich immer als eine Art Schiedsrichter, dessen ausschließliche Aufgabe es war, zu überwachen, dass die Parteien die Regeln einhielten und die Gebote der Fairness nicht überschritten. ${ }^{331}$ Die Wahrheitsfindung als solche lag bei den Parteien und sollte sich aus der Einhaltung der prozessualen Regeln „quasi von selbst“ ergeben. ${ }^{332}$ Dieser Anspruch wird durch die CPR 1998 zwar nicht aufgegeben, aber doch grundlegend modifiziert. R. 1.1 CPR 1998 stellt die gerechte Durchführung des Verfahrens als vorrangige Maxime heraus. ${ }^{333}$ Um diese Zielsetzung zu verwirklichen, geben die CPR 1998 dem Gericht Mittel an die Hand, damit es das Verfahren aktiv und zielstrebig leiten kann (sog. Case management). ${ }^{334}$ Dabei soll der Prozess nach wie vor nicht inquisitorischen, sondern kooperativen Grundsätzen folgen. Dass das Verfahren jedoch in die Verantwortung des Gerichts verlagert wird, verdeutlichen insbesondere drei Punkte.

1. Das Gericht legt eine umfassende Gerichtsakte an, sobald das Verfahren anhängig ist, um seinen verfahrensleitenden Aufgaben nachzukommen. ${ }^{335}$ Dies war nach den RSC gerade nicht der Fall, da der Richter völlig unvorbereitet in die Verhandlung kommen sollte, damit sichergestellt war, dass er sich nicht vorher schon ein Urteil gebildet hatte. ${ }^{336}$ Nach den neuen Regeln müssen Kopien aller Schriftsätze und Dokumente, die zwischen den Parteien im Vorverfahren ausgetauscht werden, zu den Gerichtsakten gegeben werden. ${ }^{337}$

\footnotetext{
${ }^{325}$ Zur Reformbedürftigkeit siehe Michalik, S. $117 \mathrm{ff}$

${ }^{326}$ White Book 1995, Preface IX; vgl.auch Malterer, S. 5 ff.

${ }^{327}$ Sog. Woolf-Report. Umfassende Darstellung in deutscher Sprache bei Malterer, S. 94 ff.

${ }^{328}$ Sobich, JZ 1999, S. 775, 776

${ }^{329}$ Blackstone's CPR, Preface V

${ }^{330}$ Glasser, 56 (1993) M.L.R. S. 307 ff.

${ }^{331}$ So zum Beispiel Lord Denning in Burmah Oil Co. v. Governor and Co. of the Bank of England [1971] 1 W.L.R. S. 473, 494: „In litigation as in war, if one side makes a mistake, the other can take advantage of it. No holds are barred."

${ }^{332}$ Sobich, JZ 1999, S. 775, 776

${ }^{333}$ r. 1.1 CPR 1998: ...overriding objective of enabling the court to deal with cases justly.

${ }^{334}$ r. 1.4 CPR 1998: The Court must further the overriding objectiv by actively managing cases.

${ }^{335}$ Blackstone's CPR, S. 68; O'Hare/ Hill, Rn. 1.021

${ }^{336}$ S. o. B.II.2.a.ff.

${ }^{337}$ Blackstone's Civil Practice, Rn. 15.5
} 
2. Die Entscheidung, welche Fragen beweisbedürftig sind und welche Beweismittel hierzu verwandt werden sollen, liegt nunmehr in der Hand des Richters, r. 32.1 CPR 1998. Er hat auch die Möglichkeit, das Recht zum Kreuzverhör einzuschränken, r. 32.1 (3) CPR 1998. Des Weiteren sind Sachverständige grundsätzlich nicht mehr den Parteien, sondern dem Gericht verpflichtet, r. 35.3 CPR $1998^{338}$, obwohl sie weiterhin von den Parteien benannt werden. ${ }^{339}$

3. Bei Verstößen gegen Auflagen des Gerichts, oder wenn vom Gericht gesetzte Fristen nicht eingehalten werden, ermächtigen die CPR 1998 den Richter, Sanktionen zu verhängen, auch ohne dass die andere Partei dies beantragt hat, rr. $3.4 ; 3.5 ; 3.7 ; 3.8$ CPR 1998.

Als weitere große Änderung stellt sich die Einteilung der verschiedenen Klagen in Tracks dar. Nach Austausch der Schriftsätze nimmt der Richter, der mit der Betreuung des Vorverfahrens befasst ist, eine Bewertung des Falles auf Grundlage der Schriftsätze und eines von den Parteien ausgefüllten Fragebogens sowie den gesetzlich vorgegebenen Kriterien vor, r. 26.8 CPR 1998. Die Einteilung in die einzelnen Tracks, nach denen sich das weitere Verfahren bestimmt, erfolgt im Wesentlichen nach dem Streitwert und Umfang bzw. Schwierigkeitsgrad des Falles. Einfach gelagerte Fälle bis 5000 GBP werden im Small claims track verhandelt, Part 27 CPR 1998. ${ }^{340}$ Klagen mit Streitwerten zwischen 5000 und 15.000 GBP folgen dem Fast track, sofern sie nicht besonders schwierig oder umfangreich sind, Part 28 CPR 1998. Alle übrigen Fälle fallen unter das Multi-track Verfahren.

Schließlich wurde mit den CPR 1998 die Terminologie modernisiert. Dabei handelt es sich allerdings um rein kosmetische Änderungen mit dem Ziel, die Rechtssprache von lateinischen und mittelalterlichen Begriffen zu befreien und damit für den Laien verständlicher zu machen. Als wesentliche Änderungen sind zu vermerken, dass nunmehr der Kläger Claimant, sowohl Writ als auch Summons jetzt Claim form und die Klage nicht mehr Action, sondern Claim heißt. Die Pleadings werden einheitlich als Statements of case bezeichnet und sowohl Statement als auch Particulars of claim fallen unter den gemeinsamen Begriff Particulars of claim. $^{341}$

\section{Die Änderungen bei der Verfahrenseinleitung}

Das Verfahren wird mit Hilfe einer Claim form eingeleitet, die der Kläger bei Gericht einreicht. Bei der Ausstellung der Claim form handelt es sich um eine Gerichtshandlung, nicht mehr um eine reine Parteihandlung. Die Claim form wird vom Gericht gesiegelt und mit der Verfahrensnummer versehen, das sogenannte Issue of the claim form (Ausstellung). Mit der Ausstellung wird die Verjährung des Anspruches unterbrochen, para 4.1 PD 7. ${ }^{342}$ Für die Frage, welches Gericht

\footnotetext{
${ }^{338}$ Vgl. auch Rumberg/Eicke, RIW 1998, S. 19, 20 f.

${ }^{339}$ r. 35.7 bestimmt, dass grundsätzlich nur ein Sachverständiger beauftragt werden soll. Sollten beide oder mehrere Parteien Sachverständige beauftragen wollen, sind sie angehalten sich auf einen zu einigen. Gelingt dies nicht, bestimmt das Gericht einen Gutachter. Nach rr. 35.6 \& 7 sind Sachverständige nur noch bei der mündlichen Verhandlung zu hören, wenn die Gerechtigkeit dies erfordert. Grundsätzlich wurde hier ein Schriftlichkeitsprinzip eingeführt.

${ }^{340}$ Ausnahmen hierzu stellen Personal injury- sowie gewisse Mietrechtsstreitigkeiten dar, bei denen die Streitwertgrenze bei 1000 GBP liegt, r. 27.1 (2).

${ }^{341}$ Siehe dazu Blackstone's CPR, S. 90

${ }^{342}$ O'Hare/ Hill, Rn. 9.006
} 
gem. Art. 21 EuGVÜ zuerst angerufen wurde, ist der Zeitpunkt der Zustellung der Claim form ausschlaggebend. ${ }^{343}$

\section{a. Part 7 Verfahren}

Die Mehrzahl der Fälle wird mittels einer Part 7 Claim form eingeleitet, welche funktional dem Writ unter den RSC/CCR entspricht.

\section{aa. Claim form}

Bei der Claim form handelt es sich um ein Formblatt ${ }^{344}$, das bei Gericht erhältlich ist. Dieses hat der Kläger auszufüllen. Auf Seite eins des Formulars muss das Gericht eingetragen werden, bei Klagen am High Court soll auch die Angabe der Abteilung erfolgen. Darüber hinaus sind Namen und Anschrift ${ }^{345}$ der beteiligten Parteien einzutragen, paras. 2.2 und 2.3 PD 16, und ihre Funktion, wenn sie als Vertreter im weitesten Sinne auftreten, r. 16.2 (3) CPR 1998. Unter der Überschrift Brief Details of Claim muss in knappen Worten die Natur des Anspruchs dargelegt werden. ${ }^{346}$ Dieser Eintrag entspricht ungefähr dem General indorsement $^{347}$ auf dem Writ unter den alten Regeln. ${ }^{348}$ Nach den Brief Details of Claim ist der begehrte Rechtsbehelf (Remedy) einzutragen. ${ }^{349}$ In Abkehr von der früheren Praxis kann das Gericht nunmehr alle Rechtsbehelfe zusprechen, die dem Kläger zustehen, selbst wenn dieser vergessen hat, den Antrag auf der Claim form einzutragen. ${ }^{350}$ Diese Neuerung zielt in erster Linie auf die Fälle ab, in denen Kläger ohne rechtliche Vorbildung oder anwaltliche Beratung eine Claim form ausfüllen. ${ }^{351}$ Wird auf eine Geldschuld geklagt, muss die Claim form eine Angabe der Höhe enthalten (Statement of value). ${ }^{352}$ Das Statement of value weist den eingeklagten Betrag ohne Zinsen aus. ${ }^{353}$ Die Summe, inklusive Zinsen wird in das Kästchen mit dem Titel ,amount claimed“ eingetragen. ${ }^{354}$ Diese Regel soll nicht nur für Klarheit sorgen, sondern es auch dem Gericht einfacher machen, die Klage in eines der Tracks einzuordnen. ${ }^{355}$ Klagt der Kläger auf eine nicht bezifferte Summe, muss er die Summe, die er vernünftigerweise erwarten kann, an die Tracks angepasst, anführen. ${ }^{356}$ Allerdings ist das Gericht durch diese Angaben im Urteil nicht beschränkt, r. 16.3 (7) CPR 1998. ${ }^{357}$ Bei Klagen, die nicht auf Geld gerichtet sind, muss der Kläger „Ich weiß nicht“ eintragen. ${ }^{358}$ Wird die Claim form von einem Anwalt verfasst, muss dieser sie auch unterschreiben, para 2.1 PD 5.

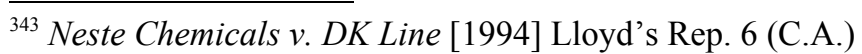

${ }^{344}$ Klagen gem. Part 7 CPR 1998 werden mit Hilfe der Form N1 eingeleitet.

${ }^{345}$ Mangels weiterer Angaben, ist davon auszugehen, dass die Rechtsprechung, soweit sie noch $\mathrm{zu}$ den RSC/CCR ergangen ist, diesbezüglich weiter gilt.

${ }^{346}$ r. 16.2 (1) (a) CPR 1998: contain a concise statement of the nature of the claim

${ }^{347}$ S. dazu oben B.II.2.a.aa.

${ }^{348}$ Blackstone's CPR, S. 91

${ }^{349}$ r. 16.2 (1) (b) CPR 1998: specify the remedy which the claimant seeks

${ }^{350}$ Blackstone's CPR, S. 91

${ }^{351}$ O'Hare/ Hill, Rn. 12.012

${ }^{352}$ r. 16.2 (1) (c) CPR 1998: where the claimant is making a claim for money, contain a statement of value...

${ }^{353}$ O'Hare/ Hill, Rn. 12.012

${ }^{354}$ O'Hare/ Hill, Rn. 12.012

${ }^{355}$ Blackstone's CPR, S. 91

${ }^{356}$ D.h. bspw. bei Fast track claims: „mehr als 5000 aber nicht mehr als 15.000 GBP“.

${ }^{357}$ O'Hare/ Hill, Rn. 12.013

${ }^{358}$ O’Hare/ Hill, Rn. 12.013
} 


\section{bb. Particulars of claim}

Auf der Rückseite der Claim form können die Particulars of claim eingetragen werden. Da der Platz jedoch sehr begrenzt ist, ist es oft von Vorteil, die Particulars of claim gesondert abzufassen, r. 7.4 (1) CPR 1998, und mit oder nach der Claim form zuzustellen. ${ }^{359}$ Trägt der Kläger die Particulars nicht auf der Claim form ein, müssen sie den Namen des Gerichts, das Aktenzeichen, den Titel des Verfahrens $^{360}$ sowie eine zustellungstaugliche Anschrift des Klägers enthalten, para. 3.8 PD 16. Werden die Particulars erst nach der Claim form zugestellt, ist letztere mit einem diesbezüglichen Hinweis zu versehen, r. 16.2 (2) CPR 1998.

Die Particulars müssen einen knappen und präzisen Vortrag der Tatsachen enthalten, auf die der Kläger seinen Anspruch stützt. ${ }^{361}$ Dabei wird diskutiert, ob das Erfordernis, r. 16.2 (1) (a) CPR 1998, dass die Claim form ,a concise statement of the nature of the claim ${ }^{362 "}$ und die Particulars ,, a concise statement of the facts on which the claimant relies" enthalten müssen, dergestalt zu interpretieren ist, dass zwingend auch schon in diesem Stadium Beweis angetreten werden muss. ${ }^{363}$

Dafür soll sprechen, dass sowohl Claim form als auch Particulars mit einem Statement of truth $^{364}$ versehen werden müssen und damit automatisch impliziert würde, die Claim form bzw. Particulars enthielten Beweise oder Beweisantritte. ${ }^{365}$ Gegen diese Ansicht ist jedoch anzuführen, dass die Voraussetzungen für eine ordnungsgemäß ausgefüllte Claim form bzw. Particulars gerade nicht verlangen, das diese Beweisantritte enthalten sollten. Die Normen zu Claim form und Particulars regeln die Anforderungen positiv. Es handelt sich bei r. 16.2 (1) (a) CPR 1998 lediglich um die positivrechtliche Fixierung althergebrachter Pleading-Regeln, die Präzision, Knappheit, aber auch ausreichenden Umfang erfordern. ${ }^{366}$ Das Statement of truth hat in erster Linie die Funktion, die Parteien zu disziplinieren ${ }^{367}$, es lässt sich daraus keine Pflicht zum Beweisantritt ableiten. Darüber hinaus erlaubt para. 14.3 PD 16 nunmehr erstmalig im englischen Zivilprozess das Anführen von Beweisen bereits im Vorverfahren. Bestünde ohnehin schon eine Pflicht dazu, wäre para 14.3 PD 16 sinnlos.

Obwohl die CPR 1998 sich einer etwas anderen Sprache bedienen als die RSC, stellen sie also keine grundsätzliche Abkehr von der gewohnten Praxis der Pleadings dar, in den Particulars, wie auch schon im Statement of claim unter RSC O. 18 r. 7 (1), nur Tatsachenbehauptungen (Material Particulars) anzuführen, jedoch keine Beweise (Evidence), die die Tatsachen belegen sollen. ${ }^{368}$ Dies folgt auch weiterhin aus der Trennung von Vor- und Hauptverfahren, wonach Beweiserhebung ausschließlich ins Hauptverfahren gehört.

Klagt der Kläger Zinsen mit ein, muss er - wie vor den CPR 1998 - den Rechtsgrund, auf den er den Zinsanspruch stützt, r. 16.4 (1) (a) CPR 1998, und bei einer Klage auf eine bezifferte Geldsumme zusätzlich die Zinshöhe, den Berechnungszeitraum, die gesamte Zinssumme für den Berechnungszeitraum sowie die tägliche Summe für die Zeit nach dem Berechnungszeitraum aufführen,

\footnotetext{
${ }^{359}$ Zur Zustellung siehe unten B.III.3.

${ }^{360}$ Namen der Parteien und Bezeichnung als Kläger bzw. Beklagter.

${ }^{361}$ r. 16.4 (1) (a): a concise statement of the facts on which the claimant relies; siehe dazu May/Scott, Rn. 16.4.1

${ }^{362}$ S.o. B.III.2.a.aa.

${ }^{363}$ Blackstone's Civil Practice, Rn. 24.17

${ }^{364}$ Versicherung der Richtigkeit der Angaben; S. dazu B.III.2.c.

${ }^{365}$ Blackstone's Civil Practice, Rn. 24.17

${ }^{366}$ Blackstone's Civil Practice, Rn. 24.17

${ }^{367}$ S. dazu B.III.2.c.

${ }^{368}$ Blackstone's Civil Practice, Rn. 24.3; Blackstone's CPR, S. 93
} 
r. 16.4 (1) (b) CPR 1998. Angaben wie ,pursuant to statute ${ }^{6369}$ oder ,im Ermessen des Gerichts" sind nicht ausreichend. ${ }^{370}$

Wird auf Herausgabe einer Sache geklagt, müssen die Particulars den Wert der Sache angeben, para. 8.2 PD 16. Stützt sich der Kläger auf eine schriftliche Vereinbarung, soll er eine Kopie des Dokumentes beifügen, para. 8.3 PD 16. Handelt es sich um eine mündlich oder durch konkludentes Handeln geschlossene Vereinbarung, sollen die genauen Umstände sowie die gesprochenen Worte, para. 8.4 PD 16, bzw. vorgenommenen Handlungen, para. 8.5 PD 16, angeführt werden. ${ }^{371}$

Schließlich muss der Kläger, sofern er seinen Anspruch darauf stützen will, folgende Punkte schon in den Particulars nennen, para. 10. 2 PD 16: fraud, illegality, misrepresentation, breach of trust, notice or knowledge of fact, unsoundness of mind or undue influence, wilful default, facts relating to mitigation of loss or damages. ${ }^{372}$ Diese Punkte in den Particulars zu nennen, war auch unter den RSC schon üblich, aber nicht vorgeschrieben. ${ }^{373}$

Eine radikale Abkehr vom bisherigen System scheint para. 14.3 PD 16 darzustellen. ${ }^{374}$ Danach steht es den Parteien frei, schon in den Statements of case Rechtsausführungen vorzunehmen, Zeugen zu benennen und Dokumente beizufügen, die für das Verfahren von Wert sein können, para 14.3 PD 16. Obwohl es anerkannte Praxis bleiben dürfte, keine Beweise im Vorverfahren anzuführen, erlaubt para. 14.3 PD 16 dies nunmehr erstmalig. Damit ist die strikte Trennung von Tatsachenbehauptung und Beweis formal aufgehoben. Allerdings gab es auch unter den RSC/CCR immer erhebliche Grauzonen zwischen sog. Material Particulars und Evidence ${ }^{375}$. Diese Grauzonen werden durch para. 14.3 PD 16 beseitigt. In der Praxis hat diese Vorschrift bisher kaum zu Änderungen im Verhalten der Parteien geführt. ${ }^{376} \mathrm{Im}$ Verfahren vor dem Commercial Court werden die Parteien sogar ausdrücklich aufgefordert, keine Beweise im Vorverfahren anzuführen. ${ }^{377}$ Auch die Möglichkeit Rechtsansichten anzuführen, ist kein so großer Schritt, wie es auf den ersten Blick scheint, insbesondere, da die Gerichte an Rechtsansichten der Parteien nicht gebunden sind.

Demnach stellt sich die Situation so dar, dass die Parteien zwar schon im Vorverfahren Beweis antreten können, hierzu aber nicht verpflichtet sind. Bis dato wird von der Möglichkeit auch nur spärlich Gebrauch gemacht. Das Verhalten der

\footnotetext{
369 „,nach Gesetz“

${ }^{370}$ Blackstone's CPR, S. 94 Streit stehende Grundstück genau identifiziert werden.

${ }^{373}$ Blackstone's CPR, S. 94

${ }^{374}$ Blackstone's CPR, S. 94

${ }^{375}$ Blackstone's Civil Practice, Rn. 24.17

${ }^{376}$ Bramley/Gouge, S. 53

${ }^{377}$ Commercial Court Guide, Section C 1.2 i
}

${ }^{371}$ Weitere spezielle Punkte finden sich in PD 16, z.B. für Verbraucherkreditstreitigkeiten, die ausnahmsweise am High Court anhängig gemacht werden sollen, para. 8.6 PD 16, oder wenn sich der Kläger zum Beweis einer Tatsache auf eine Verurteilung des Beklagten in einem Strafverfahren stützen will, para. 10.1 PD 16. Nach para. 8.1 PD 16 muss bei Klagen auf eine einstweilige Verfügung (Injunction) oder Feststellung (Declaration) im Zusammenhang mit Grundbesitz das im

372 arglistige Täuschung, Verstoß gegen Verbotsgesetze, Falschauskunft, Pflichtverletzung in Bezug auf einen Trust, Kenntnis des Beklagten von Tatsachen, die der Kläger behauptet, Geistesgestörtheit oder unzulässige Einflussnahme, vorsätzliches Unterlassen trotz bestehender Handlungspflicht, Umstände im Zusammenhang mit Schadensminderung. Der letzte Punkt ist problematisch, da das Vorbringen einer Schadensminderungspflicht des Klägers traditionell beim Beklagten liegt. Nach O'Hare/ Hill, Rn. 12.030 hat der Kläger hierzu nur Angaben zu machen, wenn der Punkt bereits im vorprozessualen Schriftverkehr vom Beklagten aufgegriffen wurde. 
Parteien hat sich insoweit im Vergleich zu den RSC kaum geändert. ${ }^{378}$ Die Gefahr, fehlerhafte Statements of case bzw. Pleadings abzugeben, weil sich Material Particulars von Evidence nicht trennen lassen, ist hingegen nunmehr gebannt.

Die Particulars können alternative Behauptungen enthalten, dürfen aber nur im Rahmen eines Alternativverhältnisses widersprüchlich sein. Wird durch zu viele Alternativbehauptungen die Aussage unklar und erfüllen die Particulars damit ihren Zweck nicht mehr, läuft der Kläger Gefahr, durch eine nachteilige Kostenentscheidung oder im schlimmsten Fall durch Nicht-Zulassung einzelner Punkte oder des gesamten Statements ${ }^{379}$ bestraft zu werden. ${ }^{380}$

Wurden die Particulars von einem Anwalt (solicitor, barrister) verfasst, ist seine Unterschrift erforderlich, para 2.1 PD 5, ${ }^{381}$ vor dem Commercial Court unabhängig vom Beruf die Unterschrift des Verfassers. ${ }^{382}$

In formaler Hinsicht müssen die Particulars weiterhin auf A4 Papier mit $3,5 \mathrm{~cm}$ Rand verfasst werden, soweit praktikabel, vollständig leserlich gedruckt oder getippt sein, wenn möglich zusammengebunden oder so fixiert sein, dass sie in Akten angelegt werden können, para 2.2 (1-3) PD 5. Andernfalls ist jede Seite mit dem Aktenzeichen zu versehen, para 2.2 (3) PD 5. Die Seiten müssen nummeriert und der Inhalt in nummerierte Abschnitte gegliedert werden, para 2.2 (4 und 5) PD 5. Neben diesen rein formalen Vorschriften bleibt es gewohnheitsrechtliche Praxis, dass Schriftsätze soweit möglich die Tatsachen chronologisch angeben und den Fall Punkt für Punkt abhandeln sollen und zwar so, dass eine Beantwortung der jeweiligen Punkte möglich ist. ${ }^{383}$ Schließlich soll jeder Gliederungsabschnitt nur eine Behauptung enthalten. ${ }^{384}$

Auf diese Behauptungen muss der Beklagte auch im Einzelnen antworten, r. 16.5 (1) CPR 1998. Er kann sie zugestehen, mit Nichtwissen bestreiten oder qualifiziert bestreiten, das heißt eine Gegenbehauptung aufstellen oder die Gründe für sein Bestreiten anführen r. 16.5 (1) (a-c) und (2) CPR 1998. Äußert er sich zu einem Punkt nicht im Einzelnen, gilt dieser als zugestanden, r. 16.5 (5) CPR 1998, es sei denn, der Beklagte hat in seiner Darstellung des Falles klar gemacht, dass er die Behauptung bewiesen haben möchte (konkludentes Bestreiten), r. 16.5 (3) (b) CPR 1998.

Es ist unklar, welche Sanktionen bei Verstoß gegen die Formvorschriften zu den Schriftsätzen drohen. Da diese Vorschriften aber den Normen zu schriftlichen Zeugenaussagen gleichen, ist davon auszugehen, dass die Sanktionen dieselben sind. Nach para. 25.1 PD 32 steht es dem Gericht frei, die formal mangelhafte Aussage zurückzuweisen und der Partei die Kosten aufzuerlegen. Es ist davon auszugehen, dass englische Gerichte auch formfehlerhafte Schriftsätze derart behandeln werden. Dabei wird die Befugnis des Gerichts wohl eher aus common $l a w^{385}$ als aus einer Analogie zu para. 25.1 PD 32 hergeleitet werden.

\footnotetext{
${ }^{378}$ Als Ausnahme davon gelten Personal Injury Fälle, wo es inzwischen üblich ist, medizinische Gutachten beizufügen; Bramley/ Gouge, S. 53.

${ }^{379}$ Noch zur alten Rechtslage: Remmington v. Scoles [1897] 2 Ch. 1

${ }^{380}$ O'Hare/ Hill, Rn. 12.032

${ }^{381}$ Blackstone's Civil Practice, Rn. 24.4

${ }^{382}$ O'Hare/ Hill, Rn. 12.004

${ }^{383} \mathrm{O}^{\prime}$ Hare/ Hill, Rn. 12.005

${ }^{384}$ O'Hare/ Hill, Rn. 12.005

${ }^{385}$ Die allgemeine Befugnis des Gerichts, mangelhafte Schriftsätze zurückzuweisen, ergibt sich für die Rechtslage vor Einführung der CPR 1998 aus Remmington v. Scoles [1897] 2 Ch. 1.
} 


\section{b. Part 8 Verfahren}

In Fällen, bei denen ein Streit über Fakten unwahrscheinlich ist oder der begehrte Rechtsbehelf dergestalt ist, dass ein normales Verfahren unangemessen umfangreich wäre, folgt das Verfahren part 8 CPR $1998 .{ }^{386}$ Meist handelt es sich dabei um Streit über eine Rechtsfrage oder die Auslegung einer Erklärung bzw. eines Dokumentes. ${ }^{387}$ Sinn der Regeln ist, für Fälle mit wenig Aufwand ein geeignetes Verfahren bereitzustellen. ${ }^{388}$ Part 8 CPR 1998 ist insoweit der Nachfolger des Verfahrens mit Originating summons unter den RSC/CCR. ${ }^{389}$

Das Verfahren wird mit Hilfe der Part 8 Claim form eingeleitet. Dabei handelt es sich wie bei der Part 7 Claim form um ein bei Gericht erhältliches Formblatt. ${ }^{390}$

Die Claim form muss den ausdrücklichen Hinweis enthalten, dass es sich um eine Klage nach part 8 handelt, r. 8.2 (a) CPR 1998. Des Weiteren muss sie unter der Überschrift Details of Claim die Frage enthalten, die der Kläger geklärt haben möchte oder den begehrten Rechtsbehelf und die Rechtsgrundlage dafür bzw. die Bestimmung in den CPR 1998 oder PD, die eine Einleitung nach part 8 vorschreibt, r. 8.2 (b) CPR 1998. Wird die Klage auf einen förmliches Gesetz (Statute) (im Gegensatz zum Common Law) gestützt, ist dieser anzuführen, r. 8.2 (c) CPR 1998. Sind Kläger oder Beklagter als Vertreter einer anderen Person am Verfahren beteiligt, hat der Kläger dies zu vermerken, r. 8.2 (d) CPR 1998. Es liegt in der Natur der Klage nach part 8, dass keine Particulars einzutragen sind. Statt dessen ist der Claim form jeglicher schriftliche Beweis (Zeugenaussagen, Gutachten) beizufügen, auf den der Kläger seine Klage stützen will, r. 8.5 (1) und (2) CPR 1998. Aber auch die in der Claim form unter Details of Claim angeführten Punkte zum Sachverhalt sind als Beweis geeignet, wenn die Claim form mit einem Statement of truth ${ }^{391}$ versehen ist, para. 5.2 PD 8. Dabei sollte der Kläger auf ausführliche Sachverhaltsdarstellungen verzichten. Der Sachverhalt sollte sich aus den mitgelieferten Zeugenaussagen ergeben. ${ }^{392}$

Das Gericht kann entweder von Amts wegen oder auf Antrag des Beklagten ${ }^{393}$ jederzeit anordnen, dass die Klage im normalen Verfahren nach part 7 weitergeführt wird, wenn es dies für angemessen hält, r. 8.1 (3) CPR 1998; para 1.6 PD 8..$^{394}$

\section{c. Statement of truth}

Nach r. 22.1 (1) (a) CPR 1998 muss jedes Statement of case inklusive der Claim form eine Versicherung der inhaltlichen Wahrheit (Statement of truth) enthalten. Dabei handelt es sich nach Ansicht von Praktikern um die substantiellste Änderung zu den RSC/CCR. ${ }^{395}$ Das Statement of truth kann entweder im Statement of case selbst abgegeben oder in einem gesonderten Dokument beigefügt werden, para. 2.3 PD 22. Das Statement of truth hat entweder die Partei selbst oder ihr Anwalt (Legal representative ${ }^{396}$ ) zu erklären und es muss die Versi-

\footnotetext{
${ }^{386}$ Für Beispiele siehe para 1.4 PD 8; Blackstone's CPR, S. 85.

${ }^{387}$ Vgl. r. 8.1 (2) und (6) CPR 1998; paras. 1.1 und 1.2 PD 8; Bramley/ Gouge, S. 60 f.

${ }^{388}$ Blackstone's Civil Practice, Rn. 13.2

${ }^{389}$ Dazu s. o. B.II.2.a.gg.

${ }^{390}$ Form N 208

${ }^{391} \mathrm{~S}$. dazu unten B.II.2.c.

${ }^{392}$ O'Hare/ Hill, Rn. 10.004

${ }^{393}$ Ausführlich zu den Gründen in r. 8.8 (1) CPR 1998 O`Hare/ Hill, Rn. 10.007.

${ }^{394}$ Blackstone's CPR, S. 85

${ }^{395}$ Bramley/ Gouge, S. 63; ebenso Aussagen verschiedener Solicitors des Dispute Resolution Departments von Baker \& McKenzie in London.

${ }^{396}$ R. 2.3 (b) CPR 1998 definiert Legal representative als Barrister, Solicitor, Angestellter eines Solicitors oder eine andere nach Courts and Legal Services Act 1990 zur Vertretung befugte Per-
} 
cherung enthalten, dass der Unterzeichner bezüglich der Angaben auf dem Statement of case glaubt, dass es sich dabei um die Wahrheit handelt. ${ }^{397}$ Unterzeichnet der Anwalt das Statement of truth, versichert er damit im Namen des Mandanten, dass dieser die Angaben für die Wahrheit hält, para. 3.7 PD 22. Dabei genügt es nicht, dass der Anwalt glaubt, der Mandant sei gutgläubig und sage die Wahrheit (bona fide). Er muss sich dies ausdrücklich versichern lassen. ${ }^{398}$ Mit Abgabe des Statement of truth versichert der Anwalt in eigenem Namen ${ }^{399}$, dass er vom Mandanten zur Abgabe bevollmächtigt ist, dem Mandanten die Bedeutung des Statement of truth verdeutlicht hat und der Mandant sich gegebenenfalls wegen Contempt of court strafbar macht, r. 32.14 (1) CPR 1998, sofern er die Angaben nicht in gutem Glauben gemacht hat, para 3.8 PD 22.

Ein verifiziertes Statement of case kann in allen Anhörungen, die nicht in der Hauptsache stattfinden ${ }^{400}$, als Beweis genutzt werden, r. 32.6 (2) (a) CPR 1998.

Wird ein Statement of case ohne Statement of truth abgegeben, bleibt es wirksam, entfaltet jedoch keine Beweiswirkung, r. 22.2 (1) (a und b) CPR 1998. Holt die Partei die Versicherung nicht nach, kann das Gericht von Amts wegen oder auf Antrag der anderen Partei anordnen, dass ein Statement of truth abgegeben wird, r. 22.4 CPR 1998. Ebenso kann das Gericht das Statement of case für unwirksam erklären, r. 22.2 (2) CPR 1998. In der Regel wird das Gericht die Unwirksamkeit anordnen, falls die säumige Partei die Abgabe des Statement of truth nicht innerhalb einer gesetzten Frist nachholt, para. 4.2 PD 22. ${ }^{401}$

Es ist ratio der Vorschriften zum Statement of truth, Parteien schon frühzeitig zu zwingen, die Substanz des Anspruches zu prüfen und die wesentlichen Punkte herauszustellen. Jemand soll Verantwortung für den Inhalt der Statements of case übernehmen. ${ }^{402}$ Es soll verhindert werden, dass leichtfertig Tatsachen vorgetragen $^{403}$ und Klagen aus rein taktischen Gründen ${ }^{404}$ aufrecht erhalten werden. Damit sollen Geld, Zeit und Gerichtsressourcen ${ }^{405}$ gespart werden. ${ }^{406}$

\section{3. Änderungen bei der Zustellung}

Die Zustellung von Schriftsätzen und anderen Dokumenten ist einheitlich in part 6 CPR 1998 und den dazugehörigen Practice Directions geregelt.

\section{a. Zustellung durch das Gericht}

Grundsätzlich werden gem. r. 6.3 (1) CPR 1998 Schriftstücke, die vom Gericht ausgestellt werden, insbesondere die Claim form, auch vom Gericht zugestellt, ${ }^{407}$ es sei denn, die Ausnahmen in r. 6.3 (1) (a-d) CPR 1998 sind einschlägig. Die wichtigste Ausnahme findet sich in r. 6.3 (1) (b) CPR 1998, wonach eine Partei

\footnotetext{
son, die mit der Vertretung beauftragt wurde.

${ }^{397}$ [I believe] [the (claimant or as may be) believes] that the facts stated in this [name document being verified] are true. para. 2.2 PD 22

${ }^{398}$ Blackstone's Civil Practice, Rn. 23.12

${ }^{399}$ O'Hare/ Hill, Rn. 12.004

${ }^{400}$ r. 32.6 (2): ,hearings other than the trial“"

${ }^{401}$ Bramley/ Gouge, S. 66

${ }^{402}$ O'Hare/ Hill, Rn. 12.004

${ }^{403}$ Blackstone's Civil Practice, Rn. 23.13

${ }^{404}$ Bspw. zur Stärkung der eigenen Verhandlungsposition bei Vergleichsverhandlungen.

${ }^{405}$ Kritisch zur drohenden Sanktion des Strafverfahrens wegen Contempt of Court: Blackstone's Civil Practice, Rn. 23.14.

${ }^{406}$ Bramley/ Gouge, S. 67

${ }^{407}$ Insoweit wurde die Praxis nach den CCR übernommen.
} 
das Gericht davon in Kenntnis setzen kann, dass sie die Zustellung selbst vornimmt. In diesem Fall bleibt das Gericht inaktiv.

Obwohl das Gericht, wenn es die Zustellung vornimmt, in der Wahl der Mittel frei ist, r. 6.3 (2) CPR 1998, ${ }^{408}$ geschieht dies in der Regel durch Post erster Klasse, para. 8.1 PD 6. Gelingt die Zustellung einer Claim form, wird der Kläger benachrichtigt, r. 6.14 CPR 1998. ${ }^{409}$ Dieses Certificate of service muss das Datum enthalten, an dem die Zustellung vorgenommen wurde, r. 6.10 (b) CPR 1998, das Datum, an dem die rechtliche Vermutung der Zustellung eintritt, r. 6.14 (1) CPR 1998, sowie den Hinweis, dass das Dokument nicht als unzustellbar zurückgesandt wurde, r. 6.10 (a) CPR 1998.

Bei allen Dokumenten, die das Gericht zustellt, erhält der Kläger im Falle des Misserfolges der Zustellung eine Notice of non-service, r. 6.11 CPR 1998. Diese enthält einen Hinweis darauf, mit welchem Mittel die Zustellung versucht wurde. Sobald das Gericht die Notice zugesandt hat, entfällt die Pflicht, weitere Zustellungsversuche zu unternehmen. Dies obliegt dann dem Kläger, r. 6.3 (1) (a) CPR 1998; para. 8.2 PD 6.

\section{b. Parteizustellung}

Stellt das Gericht nicht zu oder misslingt der Zustellungsversuch, ist es Aufgabe der Partei, die Zustellung des Dokumentes vorzunehmen. Dabei stehen der Partei wie auch dem Gericht in obigem Falle ${ }^{410}$ verschiedene Möglichkeiten zur Verfügung.

Das Dokument kann persönlich zugestellt werden, r. 6.4 CPR 1998. Dabei handelt es sich um die traditionelle Methode im Common Law, die sich immer noch großer Beliebtheit erfreut, da in der Regel ein Streit über den Zugang ausgeschlossen ist. Bei der persönlichen Zustellung muss das Dokument beim Adressaten hinterlassen ${ }^{411}$ und dieser über den Inhalt des Briefes informiert werden. Weigert er sich, den Brief anzunehmen ${ }^{412}$, ist es ausreichend, den Brief so weit wie möglich in seinen Machtbereich zu bringen. ${ }^{413}$ Wird der Adressat von einem Anwalt vertreten, so sind diesem alle Dokumente zuzustellen, r. 6.4 (2) CPR 1998. Eine Ausnahme gilt insoweit für die Claim form: Sie kann dem Anwalt nur zugestellt werden, wenn er diesbezüglich empfangsbevollmächtigt ist und dies dem Kläger bekannt gemacht hat. ${ }^{414}$ Wird eine Partnership verklagt, ist die Claim form einem Partner oder leitenden Manager zuzustellen, r. 6.4 (5) (a und b) CPR 1998. Wird eine Kapitalgesellschaft verklagt, ist die Claim form einem Angestellten in einer senior position ${ }^{415}$ zuzustellen, r. 6.4 (4) CPR 1998.

Auch die Parteizustellung kann per Post erster Klasse erfolgen, r. 6.2 (1) (b). Erstaunlicherweise wird eine Zustellung per eingeschriebenem Brief nicht

\footnotetext{
${ }^{408}$ Siehe zu den anderen Zustellungsmöglichkeiten unten B.III.3.b.

${ }^{409}$ Dies gilt nur für die Claim form.

${ }^{410}$ Parteien sind nicht an para.8.1 PD 6 gebunden, wonach das Gericht gewöhnlich mit Post erster Klasse zustellt.

${ }^{411}$ r. 6.4 (3) CPR 1998: A document is served personally on an individual by leaving it with that individual.

${ }^{412}$ Zur alten Rechtslage, die insoweit wohl fortgilt: Thomson v. Pheney (1832) 1 Dowl Pr Cas 441

${ }^{413}$ Z. B. reicht es aus, den Brief vor die Füße des Adressaten zuwerfen. O’Hare/Hill, Rn. 8.002

${ }^{414}$ Smith v. Probyn (2000) The Times, 29. 03. 2000: rr. 6.13 (2) und 6.4 (2) CPR 1998 sind zusammen zu lesen.

${ }^{415}$ Nach para. 6.2 PD 6 ist das der Direktor (director), Schatzmeister (treasurer), Syndicus (secretary), Geschäftsführer (chief executive), Manager (manager) oder jedes andere Vorstandsmitglied (officer).
} 
erwähnt. Ob es sich dabei trotzdem um ein taugliches Mittel handelt, bleibt abzuwarten. ${ }^{416}$

Wird persönlich oder per Post zugestellt und hat der Zustellungsadressat keine Zustellungsadresse angegeben, ist die Claim Form bei einer natürlichen Person an ihren üblichen oder den letzten bekannten Wohnort (residence) bzw. Geschäftsort (place of business) zuzustellen. Bei juristischen Personen, die in England oder Wales registriert sind, erfolgt die Zustellung an die Hauptniederlassung oder einen Geschäftsort, der mit der Klage zu tun hat. Bei allen anderen juristischen Personen kann an irgendeinen Ort, von dem aus die Person Geschäfte betreibt, zugestellt werden, r. 6.5 (6) CPR 1998.

Zustellung per Document Exchange (DX) ist grundsätzlich zulässig, para. 2.1 PD 6, wenn und soweit der Adressat dies im Vorfeld akzeptiert hat. Für eine derartige Annahme reicht die Aufführung einer DX-Nummer im Briefkopf, vorausgesetzt, die Partei hat nicht schriftlich darauf hingewiesen, dass die Zustellung von Schriftsätzen über DX nicht akzeptiert wird, para. 2.1 (3) PD 6.

Die Zustellung per Fax ist zulässig, wenn und soweit die Partei oder ihr Anwalt sich schriftlich mit der Zustellung per Fax einverstanden erklärt und eine Nummer angegeben hat, para. 3.1 (1) ( $a$ und b) PD 6. Dies wird bei Anwälten vermutet, wenn eine Faxnummer im Briefkopf aufgelistet ist und kein schriftlicher Hinweis erfolgt ist, dass diese Nummer nicht für die Zustellung gedacht ist, para. 3.1 (3) PD $6 .{ }^{417}$ Wird ein Dokument einer Privatperson per Fax zugestellt, muss diese die Nummer ausdrücklich und schriftlich zum Empfang von Schriftsätzen mitgeteilt haben. ${ }^{418}$

Andere elektronische Medien (insbesondere E-Mail) können für die Zustellung genutzt werden, wenn beide Parteien durch Anwälte vertreten werden, diese sich im Vorfeld darauf geeinigt haben, das Medium für die Zustellung zu akzeptieren und entsprechende Adressen angegeben haben, para. 3.3 PD 6.

Wird ein Dokument per Fax oder auf elektronischem Wege zugestellt, muss keine Papierversion nachgereicht werden. Wird dies jedoch unterlassen, und es entsteht ein Streit über den Empfang des Dokumentes, hat das Gericht die Tatsache, dass keine Kopie gesandt wurde, bei seiner Entscheidung in Betracht zu ziehen, para. 3.4 PD 6.

Für jede der aufgeführten Zustellungsformen normiert r 6.7 (1) CPR eine gesetzliche Vermutung für einen bestimmten Tag, an dem die Zustellung als erfolgt gilt. ${ }^{419}$ Die Vermutung ist widerlegbar. ${ }^{420}$

Wenn ein guter Grund vorliegt, kann das Gericht auf Antrag eine alternative $\mathrm{Zu}$ stellungsmethode anordnen. ${ }^{421}$ Hierbei kommt jede Methode in Betracht, die dem Gericht durchführbar erscheint. ${ }^{422}$ Insbesondere die Zustellung an eine Person, die mit dem Beklagten in regelmäßigem Kontakt steht, ist ein taugliches Mittel. Aber auch eine öffentliche Zustellung, z. B. durch Zeitungsanzeigen kann in Extremfällen angebracht sein. ${ }^{423}$

\footnotetext{
${ }^{416}$ O'Hare/ Hill, Rn.8.004

${ }^{417}$ O'Hare/ Hill, Rn. 8.007

${ }^{418}$ Molins plc v. GD SpA [2000] 1 W.L.R. 1741; Blackstone's Civil Practice, Rn. 15.13

${ }^{419}$ Bei Post erster Klasse ist dies z.B. der zweite Werktag nach Aufgabe zur Post.

${ }^{420}$ Vgl. Barclays Bank of Swaziland Ltd. v. Hahn [1989] 1 W.L.R. 506 (H.L.) noch zu RSC O. 10

r. 1 (3) (a)

${ }^{421}$ r. 6.8 (1) CPR 1998: „Where it appears to the court that there is agood reason..."

${ }^{422}$ O'Hare/ Hill, Rn. 8.014

${ }^{423}$ O'Hare/ Hill, Rn. 8.014
} 
Der Kläger kann die Claim form nur innerhalb einer Frist von vier Monaten nach Ausstellung zustellen, r. 7.5 (2) CPR 1998. ${ }^{424}$ Tut er dies nicht und beantragt auch keine Verlängerung, verfällt die Claim form. Je nach Methode der Zustellung gelten widerlegbare ${ }^{425}$ gesetzliche Vermutungen für den Zugang. ${ }^{426}$ Werden die Particulars of claim nicht zusammen mit der Claim form zugestellt, müssen sie dieser innerhalb von 14 Tagen folgen, spätestens jedoch mit Ablauf der Vier-Monats-Frist für die Claim form dem Beklagten zugestellt werden, r. 7.4 (1 und 2) CPR 1998. Anträge zur Verlängerung der Frist können gestellt werden, r. 7.6 CPR 1998. Ob die Frist tatsächlich verlängert wird, steht im Ermessen des Gerichts und hängt von einer Gesamtschau der Umstände $a b$, die eine Verlängerung erforderlich machen. ${ }^{427}$ Wird die Verlängerung erst nach Ablauf der Frist beantragt, muss der Kläger zusätzlich einen guten Grund vortragen und beweisen, warum es ihm nicht möglich war, den Antrag innerhalb der Vier-MonatsFrist zu stellen. ${ }^{428}$ Erhält der Kläger eine Fristverlängerung, ohne dass der Beklagte vorher von dem Antrag in Kenntnis gesetzt wurde, kann letzterer die Anordnung der Fristverlängerung innerhalb von sieben Tagen nach Zustellung der Anordnung angreifen. ${ }^{429} \mathrm{Ob}$ der Kläger die Claim form innerhalb der vorgegebenen (ursprünglichen oder verlängerten) Frist zugestellt hat, prüft das Gericht nur auf Antrag, nicht von Amts wegen. ${ }^{430}$

Weiß der Beklagte um die Ausstellung der Claim form, kann er den Kläger auffordern, die Claim form innerhalb einer bestimmten Frist (von wenigstens 14 Tagen, r. 7.7 (2) CPR 1998) zuzustellen oder von der Klage abzusehen, r. 7.7 (1) CPR 1998. Kommt der Kläger dieser Aufforderung nicht nach, kann das Gericht auf Antrag die Klage abweisen oder eine andere Verfügung im freien Ermessen treffen, r. 7.7 (3) (a und b) CPR 1998. Diese Regel ist in erster Linie für Fälle gedacht, in denen die Reputation des Beklagten betroffen ist. ${ }^{431}$

Die Zustellung ins Ausland nach r. 6.17-6.31 CPR 1998 folgt im wesentlichen den Regeln, die unter O. 11 RSC und dem dazu entwickelten Fallrecht galten. ${ }^{432}$

\section{c. Response Pack}

Zusammen mit den Particulars of claim muss dem Beklagten das sogenannte Response pack zugestellt werden, r. 7.8 CPR 1998. Dabei handelt es sich um bei Gericht erhältliche Formulare, nämlich eine form für die Verteidigung, eine für ein Anerkenntnis und eine für die Bestätigung der Zustellung (Acknowledging service), r. 7.8 (1) (a-c) CPR 1998. Bei Part 8 claims werden nur die beiden letztgenannten Formulare beigefügt, r. 7.8 (2) (a) CPR 1998. Der Beklagte muss seine Verteidigung innerhalb von 14 Tagen nach Erhalt der Particulars an das Gericht senden, r. 15.4 (1) (a) CPR 1998. Er kann eine Bestätigung der Zustellung ausfül-

\footnotetext{
${ }^{424}$ Bei Zustellung ins Ausland sechs Monate, r. 7.5 (3) CPR 1998

${ }^{425}$ So die alte Rechtslage, es ist aber davon auszugehen, dass dies unter den CPR 1998 weiterhin gilt. Blackstone's Civil Practice, Rn. 15.22

${ }^{426}$ r. 6.7 (1) CPR 1998: Post erster Klasse und elektronische Übermittlung außer Fax: am zweiten Tag nach Absendung; DX: am zweiten Tag nach Hinterlegung; Lieferung oder Hinterlegung an genehmigter Adresse: am Tag nach der Lieferung oder Hinterlegung; Fax: Übermittlung vor 16 Uhr eines Werktages: am selben Tag, danach: am nächsten Werktag.

${ }^{427}$ Blackstone's Civil Practice, Rn. 15.3

${ }^{428}$ Blackstone's Civil Practice, Rn. 15.4; vgl. auch Vinos v. Marks and Spencer plc The Independent, 17.07. 2000

${ }^{429}$ O'Hare/ Hill, Rn. 9.010

${ }^{430}$ O'Hare/ Hill, Rn. 9.010

${ }^{431}$ O'Hare/ Hill, Rn. 9.011

${ }^{432}$ Siehe dazu oben B.II.2.a.bb.
} 
len, wenn er nicht in der Lage ist, innerhalb der Frist seine Verteidigung fertigzustellen, oder er die Zuständigkeit des Gerichts angreift, r. 10.1 (3) (a und b) CPR 1998, und diese innerhalb von 14 Tagen an das Gericht senden, r. 10.3 (1) CPR 1998. In diesem Fall verlängert sich die Frist für die Verteidigung automatisch auf 28 Tage, r. 15.4 (1) (b) CPR 1998.

\section{Zusammenfassung}

Die CPR 1998 führen ein gänzlich neues Regime im englischen Zivilprozessrecht ein. Dabei wurden gerade bei der Verfahrenseinleitung zum Teil große Änderungen vorgenommen. Bei genauerer Betrachtung stellen sich allerdings auch relativ viele Änderungen als eher kosmetischer Natur dar. Die Überwachungsaufgaben des Gerichts wurden auf das Vorverfahren ausgedehnt und teils $\mathrm{zu}$ Verfahrensleitungskompetenzen umgewandelt. Dies geschieht unter anderem durch das Führen von Gerichtsakten im Vorverfahren. Gleichzeitig wurden die Zustellungsvorschriften modernen Kommunikationsmedien angepasst. Das Vorverfahren wurde gestrafft und die Regeln für die Statements of claim (Pleadings) verschärft, um taktische Manöver $\mathrm{zu}$ vermeiden und die Verfahrensdauer $\mathrm{zu}$ kürzen. Von erheblicher Bedeutung ist die Einführung der Versicherung der Wahrheit der in den Statements of claim vorgetragenen Tatsachen. Damit wird erreicht, dass die Parteien erstmals in der englischen Geschichte dem Gericht und nicht nur einander verpflichtet sind.

In die gleiche Entwicklungsrichtung, hin zu mehr gerichtlicher Verantwortung und Einschränkung der Parteienmacht, geht die Regelzustellung durch das Gericht. Auch die Einschränkung der Dispositionsmaxime durch eine kooperative Prozessgestaltung und die Möglichkeit, über den Antrag hinaus zuzusprechen, unterstreicht diese Entwicklung.

Von geringerer Bedeutung ist hingegen bei genauerer Betrachtung die Möglichkeit, in den Statements of case Rechtsansichten vorzutragen und Beweismittel anzugeben. Ersteres bindet das Gericht nicht und die Möglichkeit vom Beweisangebot Gebrauch zu machen, wird nur sehr zurückhaltend genutzt. Zum Teil wird sogar ausdrücklich davon abgeraten.

Ob die Reform die angestrebten Ziele Effizienz, Geschwindigkeit und Gerechtigkeit verwirklicht, bleibt noch abzuwarten, die Entwicklung zu mehr staatlichen Befugnissen ist jedoch unübersehbar. 


\section{Rechtsvergleichende Betrachtung zum deutschen Recht}

Nachdem die Rechtslage in England und Wales ausführlich dargestellt wurde, soll im Folgenden die Entwicklung des deutschen Rechts skizziert und sodann abschnittsweise mit der englischen Rechtslage verglichen werden.

Das deutsche Recht, als Mutterrechtsordnung des deutschen Rechtskreises, ${ }^{433}$ stellt neben den romanischen Rechtsordnungen den großen Antagonisten zum Common Law dar. Insoweit drängt sich für einen deutschen Juristen ein Vergleich geradezu auf, um festzustellen, ob die Unterschiede der beiden Systeme tatsächlich so groß sind, wie allgemein angenommen wird.

\section{Klageeinleitung bis zum 12. Jahrhundert}

Die Entwicklung eines Zivilprozesses begann auf dem germanischen Gebiet des Kontinents erst zu fränkischer Zeit, so dass sich eine Betrachtung zu früherer Zeit mangels Betrachtungsgegenstand erübrigt. ${ }^{434}$

\section{Das Verfahren zu fränkischer Zeit}

$\mathrm{Zu}$ fränkischer Zeit standen dem Kläger zwei verschiedene Gerichte zur Verfügung. Ihm stand frei, ob er seine Klage an die Volksgerichte oder vor das Königsgericht bringen wollte.

\section{a. Verfahren vor den Volksgerichten}

Im fränkischen Recht wurde, wie auch im germanischen Recht zuvor, nicht zwischen Straf- und Privatrecht getrennt. Privatrechtliche Klagen waren vielmehr in Form einer (Privat-) Strafrechtsklage zu verfolgen. Bei Obsiegen wurde dann gleichermaßen eine Strafe ausgesprochen, wie der privatrechtliche Anspruch befriedigt.

\section{aa. Ladung}

Im fränkischen Recht hielt sich zunächst das Streitgedinge als Form der Klageeinleitung. Es handelte sich um ein zweiseitiges Rechtsgeschäft mit der Verpflichtung, zu einem bestimmten Termin vor Gericht zu erscheinen, um per Schiedsvertrag eine Streitschlichtung herbeizuführen. ${ }^{435}$

Daneben trat als einseitige Einleitungsform die mannitio. Dabei lud der Kläger unter Zeugen ${ }^{436}$ den Beklagten vor Gericht, indem er seinen Anspruch geltend machte und dem Gegner eine Frist setzte, nach deren Ablauf dieser vor Gericht zu erscheinen hatte. ${ }^{437}$

Der Gedanke, dass der Staat Unrecht zu verfolgen hatte, das einzelnen widerfuhr, führte in karolingischer Zeit zur Verfahrenseinleitung mit Hilfe amtsrichterlicher Ladung, sog. bannitio. ${ }^{438}$ Dabei wurde der zu Ladende vom Richter oder einem

\footnotetext{
${ }^{433}$ Zur Frage, ob es einen eigenen deutschen Rechtskreis gibt oder ob es sich um einen romanischdeutschen, also quasi kontinentaleuropäischen Rechtskreis handelt: Zweigert/Kötz, S. 130

${ }^{434}$ Vgl. zur Verfolgung privatrechtlicher Ansprüche im (privaten) Strafverfahren: Kollmann, S. 277; Schröder/Künßberg, S. 90; Wesel, Rn. 236.

${ }^{435}$ Brunner, Deutsche Rechtsgeschichte I S. 254

${ }^{436}$ Siegel, Geschichte, S. 65 f.

${ }^{437}$ Lex Sal. 1,3: Et ille qui alium manit cum testibus ad domum illius ambulare debet et sic eum manire debet [...].

Und jener, der einen anderen lädt, muß mit Zeugen zu dessen Haus gehen und muß ihn so laden [...]. (Übersetzung nach der Eckhardt-Ausgabe)

${ }^{438}$ S. auch Schröder/Künßberg, S. 390
} 
Boten unter Nennung des Gegners und des Klagegrundes ${ }^{439}$ und Setzen einer Frist $^{440}$ vor Gericht geladen.

Diese Form der Verfahrenseinleitung verdrängte bis zum 9. Jahrhundert die Parteiladungen. ${ }^{441}$

\section{bb. Klage}

Die Klage selbst wurde vor dem Richter in direkter Ansprache an den Gegner erhoben. ${ }^{442}$ Dabei enthielt die Klageansprache den Vorwurf an den Gegner, sich unrecht verhalten zu haben, bzw. sich in einem unrechten Zustand zu befinden. ${ }^{443}$ Ein bestimmter Antrag mußte nicht vorgebracht werden. Dieser ergab sich vielmehr aus dem Klagevorwurf. ${ }^{444}$

Formal war erforderlich, die Klage unter Einhaltung genau vorgeschriebener Haltung, bestimmter Worte und unter Beteuerung der Wahrheit des Vortrages vor einem göttlichen Wesen zu erheben. Letzteres wurde zum Teil durch Anbieten oder Leisten eines Voreides ersetzt. ${ }^{445}$ Schließlich mußte der Gegner zur ordnungsgemäßen Antwort aufgefordert werden. ${ }^{446}$

\section{cc. Rechtsfolgen fehlerhafter Klagen}

Da die Prozeßhandlungen in bestimmter Form unter Wahrung gewisser Floskeln abzugeben waren, bestand für die Parteien die Gefahr, durch einen Formverstoß die gesamte Prozeßhandlung zu Fall zu bringen. ${ }^{447}$ Diese Gefahr wurde im fränkischen Prozeß dadurch gemildert, dass Vorsprecher als Boten für die Parteien sprachen. ${ }^{448}$ Diese Vorsprecher waren im Umgang mit den erforderlichen Floskeln geübt, womit die Fehlerwahrscheinlichkeit sank. ${ }^{449}$

Weiterhin galt für jedes Vorbringen das Prinzip der strengen Wortinterpretation. Da somit jede Partei an ihr Wort gebunden war, konnten fehlerhafte Erklärungen

\footnotetext{
${ }^{439}$ Lex Rom. Cur. 27,9 MGH Leges I, 5 S. 440: ...et ille, quem accusat, nec per verbo nec per sigillum ante iudicem venire noluerit ... - ... und dieser, den er anklagt, weder wegen des Wortes, noch wegen des Ladungszeichens vor Gericht erscheinen will...;

${ }^{440}$ Lex Chamav c.43 MGH Leges I, 5 S. 276: ...Ingenuus, si per comitemaut per missum bannitus fuerit infra comitatum de maxima causa, super noctes 14 ad placitum veniat. Si minor causa extiterit, super noctes 7 ad placitum veniat. - Ein Freier, der vom Grafen oder seinem Boten vorgeladen worden ist, möge bei einer größeren Sache nach 14 Nächten vor der Versammlung erscheinen. Bei kleinen Sachen möge er nach sieben Nächten kommen.

${ }^{441}$ Vgl. Bethmann-Hollweg, IV, S. 378; Brunner, Deutsche Rechtsgeschichte II, S. 449.

${ }^{442}$ Bethmann-Hollweg, I, S. 67; Brunner, Deutsche Rechtsgeschichte II, S. 455

${ }^{443}$ Siegel, Geschichte, S. 116 f.

${ }^{444}$ Siegel, a.a.O., S. 118

${ }^{445}$ Dies war bei Franken, Langobarden und Sachsen der Fall. Lex Rib. 67,5, E. Codd.A., MGH Leges I, 5 S. 257: Si quis pro hereditatem vel pro ingenuitatem certare coeperit, post malo ordine cum VI in ecclesia coniurata cum XII ad stafflo regis in circlo et ... cum verborum contemplatione coniurare studeat. Si non adimpleverit, cum legis beneficium restituat. Aut si quis eum contraprendere voluerit, aut cum arma sua a se defensare studeat ante rege aut omnem repetitionem cum legis beneficium restituat. - Wenn jemand für eine Erbschaft oder die Freiheit zu streiten beginnt, und den Vorwurf der Rechtswidrigkeit mit sechs Eideshelfern beschworen hat, möge der Beklagte einen Eid mit Zwölfen auf den Stab vor dem Königsgericht unter Beachtung bestimmter Worte leisten. Wenn er dies nicht erfüllt, erstattet er es mit einer Buße. Oder wenn er dies verhindern will, möge er sich entweder mit seiner Waffe vor dem König verteidigen oder für die gesamte Klageforderung eine Buße erstatten. Der Eid des Klägers konnte als Voreid oder mit Eideshelfern geschworen werden. Vgl. Brunner, Deutsche Rechtsgeschichte II, S. 457

${ }^{446}$ Siegel, Geschichte, S. 133

${ }^{447}$ E. Kaufmann, JuS 1961, S. 120Schröder/Künßberg, S. 90 f.; vgl. auch Hattenhauer, Rn. 85 f.; Siegel, Gefahr, S. 6; Zallinger, S. 6 ff..

${ }^{448}$ Brunner, Deutsche Rechtsgeschichte II, S. 465

${ }^{449}$ Brunner, Deutsche Rechtsgeschichte II, S. 465
} 
oder einfache Versprecher nicht verbessert werden. ${ }^{450}$ Das einmal Gesagte war für die Partei verbindlich. ${ }^{451}$

Auch diesem Mißstand wurde in fränkischer Zeit abgeholfen, indem die Parteien entscheiden konnten, ob sie das vom Vorsprecher Gesagte genehmigen und damit gegen sich gelten lassen oder verbessern wollten. ${ }^{452}$

\section{b. Verfahren vor dem Königsgericht}

Das Königsgericht fungierte als Gericht mit außerordentlicher Gerichtsgewalt, das nicht an die Grundsätze des Volksrechts gebunden war, sondern seine Entscheidungen nach Billigkeitsgesichtspunkten treffen konnte. ${ }^{453}$ Dabei konnte der König unter Umgehung der Volksgerichte jede Streitigkeit an sich ziehen.

Wurde eine Klage vor dem König erhoben, konnte dieser dem Kläger einen bedingten königlichen Befehl ${ }^{454}$ aushändigen. ${ }^{455}$ Dabei handelte es sich um einen Befehl an den Beklagten, den klägerischen Anspruch zu befriedigen oder sich zu einem festgesetzten Termin vor dem Königsgericht einzufinden, um sich zu verteidigen. ${ }^{456}$ Die Ladung stand mithin unter der Bedingung der Nichterfüllung. Sein Vorbild hatte dieser fränkische indiculus commonitorius in spätrömischen Ladungsbefehlen, auf die die königlichen Hofbeamten zurückgriffen. ${ }^{457}$

Weiterhin hatte der König die Möglichkeit, den zuständigen Richter anzuweisen, dem Kläger zu seinem Recht zu verhelfen oder den Beklagten vor den König zu bringen. ${ }^{458}$ Die schriftlichen Ladungsbefehle des Königs führten auch dazu, dass die Parteiladung von der richterlichen Ladung verdrängt wurde. ${ }^{459}$

\footnotetext{
${ }^{450}$ Siegel, Gefahr, S. 6

${ }^{451}$ Sachsenspiegel, I, 60, § 1; Brunner, Deutsche Rechtsgeschichte II, S. 463; Hattenhauer, Rn. 186; E. Kaufmann, JuS 1961, S. 120 ff.

${ }^{452}$ Bieresborn, S. 43; Schröder/Künßberg, S. 844

${ }^{453}$ Brunner, Deutsche Rechtsgeschichte II, S. 184

${ }^{454}$ sog. indiculus commonitorius

${ }^{455}$ Brunner, Deutsche Rechtsgeschichte II, S. 186

${ }^{456}$ Form. Marc. I 29, MGH Form., S. 60: Propterea presentem indecolum ad vos direximus, per quem omnio iubemus, ut si taliter agitur, de presente hoc contra iam dicto illo legibus studeatis emendare. Certe si nolueretis et aliquid contra hoc habueretis quod opponere, non aliter fiat nisi vosmet ipsi per hunc indecolum commoneti Kalendas illas proximas ad nostram veniatis presentam eidem ob hoc integrum et legale dare responso. - Deswegen richten wir an Euch vorliegenden Indiculus, mit dem wir befehlen, dass wie folgt verfahren wird: Dass Ihr Euch bemühen mögt, den Anspruch des bereits genannten zu erfüllen. Wenn Ihr dies nicht wollt und etwas dagegen einwenden möchtet, möge es nicht anders sein, als dass Ihr aufgrund dieses Indiculus communitorius an den nächsten Kalenden vor uns erscheint, um vollständig und rechtmäßig Antwort zu erteilen.

${ }^{457}$ Bieresborn, S. 41; Brunner, Deutsche Rechtsgeschichte II, S. 187

${ }^{458}$ Form. Marc. I 27, MGH Form., S. 59: ...Propterea presentem indecolum ad sanctitatem vestram direximus, per quem petimus, ut et pro nos orare dignestis et, si taliter agitur, ipso abbate vestro illo, aut clerico, presentialiter constringatis, qualiter, si ita agetur, ac causa contra iam dicto illo legibus studeat emendare. Certe si noluerit at aliquid contra hoc habuerit quod opponere, ipso illo per fideiussores posito tunc ad nostram diregire studeatis presentiam. - ...Deswegen richten wir vorliegenden Indiculus an Eure Heiligkeit, mit dem wir bitten, dass Ihr so gütig sein wollt, für uns zu beten, und wenn so gehandelt wird, Ihr Euren Abt selbst oder einen Geistlichen verpflichtet, auf welche Weise, wenn so gehandelt wurde, er sich bemühen soll, auch unsere Angelegenheit gegen den bereits genannten gemäß den Gesetzen ins Reine zu bringen. Wenn der bereits genannte dies nicht will und etwas dagegen einwenden möchte, dann möget Ihr Euch bemühen, den selbigen gestützt durch Gewährsleute vor unser Angesicht zu bringen.

${ }^{459}$ S.o. C.I.1.a.aa.
} 


\section{Klageeinleitung im Hochmittelalter}

Ab dem 13. Jahrhundert gewann das sächsische Recht stärkeren Einfluß in Deutschland. Diese Entwicklung wurde insbesondere durch den Sachsenspiegel von Eike von Repgow forciert. Im Sachsenspiegel wurde erstmalig in deutscher Sprache das bestehende Recht dargestellt. Im Anschluß an den Sachsenspiegel entstand eine umfangreiche Glossenliteratur. ${ }^{460}$ Da einige der Verfasser im römischen Recht bewandert waren, machten sich auch zunehmend römisch-rechtliche Einflüsse bemerkbar. ${ }^{461}$

\section{a. Ladung}

Die Ladung mit Hilfe der bannitio hatte sich bereits zu fränkischer Zeit durchgesetzt und blieb auch im Hochmittelalter die übliche Ladungsform.

\section{b. Klage}

Bei der Klage ergaben sich zum fränkischen Recht einige Änderungen. Zumindest terminologisch wurde eine Trennung von Straf- und Privatrechtsklagen vorgenommen. Man unterschied pinlike (=peinliche; strafrechtliche), borgerlike (=bürgerliche) und vormengde (=gemischte) Klagen, wobei bei gemischten Klagen eine bürgerliche Klage strafrechtlich enden konnte, wenn sich bspw. herausstellte, dass das Verhalten des Beklagten unter Strafandrohung stand oder umgekehrt. ${ }^{462}$ Diese Unterscheidung folgte der Unterscheidung des römischen Rechts zwischen actiones civiles und actiones criminales, ${ }^{463}$ wonach die Klagen nicht nach dem Grund für die Klage, sondern nach dem Ziel der Klage eingeteilt wurden. Auch wenn dem Beklagten bei der bürgerlichen Klage, die in Klagen um Schuld, Gut und Ungericht unterschieden wurden, keine Strafe drohte ${ }^{464}$, drehte sich der Klagevorwurf weiterhin um die Schuld des Beklagten.

\section{c. Rechtsfolgen fehlerhafter Klagen}

Im mittelalterlichen Prozeß wurde der Prozessformalismus zunächst noch verstärkt. Dabei ging man sogar so weit, dass ein bloßer Versprecher oder eine Fehler bei anderen Formhandlungen zum Verlust des gesamten Prozesses führen konnte. ${ }^{465}$ Jedenfalls wurde jeder kleinste Fehler ${ }^{466}$ mit einem Bußgeld geahndet. ${ }^{467}$ Folge dieses übertriebenen Formalismus war, dass das Vorsprecherwesen stark zunahm. ${ }^{468}$

\footnotetext{
${ }^{460}$ Insbesondere der sog. Richtsteig Landrecht, in dem Johann von Buch eine Gesamtdarstellung des Prozesses vor sächsischen Landgerichten verfasste, ist hervorzuheben.

${ }^{461}$ Köbler, S. 106 ff.

${ }^{462}$ Vgl. v. Schwerin/Thieme, S. 216; Erler/Werkmüller, II, S. 837 ff.

${ }^{463}$ Homeyer, S. 49 f.

${ }^{464}$ RLdr. 5 § 1:...dar umme wete dat alle clagen sin drirleie. De erste clage is borgerlik unde het dar umme also, dat de cleger mit dem antwerder vor der sake unde na der sake borgen bliven, unde dat erer nem dem anderen vlüchtich werden ne darf, dat is alse umme sculd unde gut unde umme anevanc. - ...darum wisse, dass es insgesamt drei Klagen gibt. Die erste Klage ist bürgerlich, und das heißt, dass Kläger und Beklagter vor wie nach der Sache Bürger bleiben und sich keiner dem anderen durch Flucht entziehen darf, also bei Klagen um Schuld, Gut und Anefang. ${ }^{465}$ Siegel,Gefahr, S. 6

${ }^{466}$ Bspw. Husten, Stammeln, Erbleichen. Fehr, S. 174; Siegel, Gefahr, S. 1

${ }^{467}$ Erler/Kaufmann, I, S.1167; v. Schwerin/Thieme, S. 216; Als Grund für dieses Schikanen wird angeführt, dass es sich bei den Bußgeldern um wichtige Einnahmequellen der Gerichtsherren handelte. Vgl. Fehr, S. 174

${ }^{468}$ Schröder/Künßberg, S. 844
} 
Eine allmähliche Abschwächung fand der Formalismus und die damit einhergehende Gefahr, den Prozeß aufgrund fehlerhafter Formalia zu verlieren, in den Städten. ${ }^{469}$ Ausgehend von Ausnahmen, die z. B. flämischen Kaufleuten gewährt wurden, ${ }^{470}$ und Verfahren vor Gerichten der Gilden und Zünfte, die weniger formstreng verhandelten, ${ }^{471}$ wurden die Sanktionen bei fehlerhaftem Vortrag allmählich auf symbolische Summen herabgesetzt. ${ }^{472}$

\section{Vergleichende Betrachtung}

$\mathrm{Zu}$ angelsächsischer Zeit in England und zu fränkischer Zeit in Deutschland folgten die Verfahrensregeln gleichen Grundsätzen. Es gab kein eigentliches Zivilverfahren. Privatrechtliche Ansprüche konnten nur im Rahmen strafrechtlicher Prozesse verfolgt werden.

Dies erklärt sich aus der Tatsache, dass es im frühen Mittelalter keine einheitlichen Rechts- und Staatssysteme gab. Die Gesellschaften waren in kleineren Gruppen organisiert und innerhalb dieser Gruppen wurden auch die Streitigkeiten geklärt. ${ }^{473}$ Die Herrscher behielten sich die Durchsetzung von Strafansprüchen vor, um ihre Herrschaftsposition zu sichern. Es hatte sich aber kein Staatsverständnis, wie es die Römer kannten, entwickelt. Infolgedessen gab es auch keine Fürsorge des Staates, die dazu hätte führen können, dass den Bürgern ein Verfahren zur Schlichtung ihrer privaten Streitigkeiten zur Verfügung gestellt wurde.

Erst im Hochmittelalter wurde im deutschen Rechtsraum eine Trennung von privat- und strafrechtlichen Klagen vorgenommen. Auf die Klageeinleitung hatte dies jedoch keine Auswirkung. Die Ladung des Gegners konnte in beiden Systemen als Privatladung durch den Kläger erfolgen. Im fränkischen Recht wurde diese Form der Ladung jedoch relativ frühzeitig durch die bannitio verdrängt. In England hielt sich die Parteiladung hingegen. Die Ladung vor das fränkische Königsgericht entsprach funktional den ersten Writs unter normannischer Herrschaft. Beide waren königliche Befehle an den Beklagten, vor Gericht zu erscheinen.

Auch die Klage folgte gleichen Grundsätzen. Sie musste einen Schuldvorwurf an den Gegner enthalten, wobei dieser in direkter Rede angesprochen wurde. Dabei hatte der Kläger sich genau vorgeschriebener Worte zu bedienen und seine gerechte Gesinnung in einem Voreid zu beschwören. Auch der extreme Wortformalismus fand sich in gleichem Maße in England wie auf dem Kontinent, wo er erst mit Ende des Mittelalters eine Abschwächung erfuhr.

Beide Verfahren hatten dieselben Wurzeln: sie beruhten auf germanischen Rechtsprinzipien. ${ }^{474} \mathrm{Im}$ Hochmittelalter, als ein starker Staat ${ }^{475}$ entstand und die kleinen, relativ autarken Gesellschaftsgruppen abgelöst wurden, entwickelte sich auch ein staatlich organisierter und kontrollierter Zivilprozess. Dies eröffnete den Untertanen die Möglichkeit, sich bei der Durchsetzung ihrer Ansprüche staatlicher Hilfe zu bedienen. Im Gegenzug unterlagen sie aber den strengen Regularien, die das Ziel hatten, die Parteien zu disziplinieren und den Prozess in einfachen durchzuführenden Bahnen zu halten. Eine entsprechende Entwicklung erfolgte in Eng-

\footnotetext{
${ }^{469}$ Bieresborn, S. 73

${ }^{470}$ Siegel, Gefahr, S. 31

${ }^{471}$ R. Schmidt, Zivilprozeßrecht S. 66

${ }^{472}$ Stutz, ZRG Germ. 38 (1917), S. 367, 369 nennt beispielhaft einen „Schoppen Wein“ als Buße.

${ }^{473}$ Vgl. dazu Kollmann, S. 276.

${ }^{474}$ Caenegem, Judges, S. 114

${ }^{475}$ Vgl. zur Entwicklung des Staatsverständnisses und den Auswirkungen auf den Zivilprozess Kollmann, S. 278
} 
land erst während der normannischen Herrschaft, die das System der kleinen angelsächsischen Volksgruppen ablöste. 


\section{Das römisch-kanonische Verfahren}

Im 13. bis 15. Jahrhundert wurde das mittelalterliche Verfahren in Deutschland vom römisch-kanonischen Verfahren verdrängt. ${ }^{476}$ Dieses Verfahren hatte sich aus einem im 11. und 12. Jahrhundert an oberitalienischen Stadtgerichten praktizierten Mischprozess entwickelt, der aus römischen und langobardischen Elementen bestand. ${ }^{477}$ Durch die Bearbeitung von Legisten und Kanonisten entstand der römisch-kanonische Prozess ${ }^{478}$, der das Zivilprozessrecht Europas entscheidend beeinflussen sollte.

\section{Klageeinleitung im römisch-kanonischen Verfahren}

Die Einleitung der Klage erfolgte in der Regel durch Einreichung eines schriftlichen Klaglibells bei Gericht ${ }^{479}$ und die Anrufung des Richters, den Beklagten zu laden, sog. aditio judicis. ${ }^{480}$

\section{a. Klaglibell und aditio judicis}

Um eine Klage zu beginnen musste der Kläger ein schriftliches Klaglibell abfassen und es bei Gericht einreichen. ${ }^{481}$ Gleichzeitig wurde der Richter mit der aditio judicis gebeten, den Beklagten zu laden.

Das Klaglibell musste Klagegrund und Klagegegenstand enthalten. Dazu wurden ein Obersatz durch den Rechtsgrund gebildet und im Untersatz die Tatsachen aufgeführt, die den Anspruch begründen sollten. Darin war auch der historische Klagegrund eingeschlossen. Bei Klagen in personam waren dabei genauere Angaben zu machen als bei dinglichen Klagen ${ }^{482}$. Schließlich folgte das petitium, welches aus der Subsumtion hervorging ${ }^{483}$ und den prozessualen Antrag darstellte. ${ }^{484}$ Der Kläger musste keine Rechtsansichten vortragen, da der da mihi factum - dabo tibi ius-Grundsatz galt. ${ }^{485}$ Ebenso fehlte im Klaglibell selber der Antrag auf Prozesseröffnung, da dieser separat mit der aditio judicis gestellt wurde. ${ }^{486}$

Das Libell wurde auf einem Formular eingetragen und musste das Datum und die Anrede des Richters enthalten. Danach folgte unter Nennung des Namens des Klägers und des Beklagten Klagegrund und -gegenstand. ${ }^{487}$

Wenn der Kläger das Klaglibell eingereicht und die aditio judicis gestellt hatte, erfolgte eine inhaltliche und formelle Prüfung des Libells durch den Richter. Diese Prüfung umfasste die Prozessfähigkeit, ggf. Vollmacht und Aktivlegitimati-

\footnotetext{
${ }^{476}$ Siehe dazu C.III.1. und 2.

${ }^{477}$ Bieresborn, S. 75

${ }^{478}$ Durch die Wiederbelebung des römischen Rechts an den oberitalienischen Universitäten ab dem 11. Jahrhundert erfuhr das Zivilprozessrecht eine tiefgreifende wissenschaftliche Bearbeitung, die von den Gerichten vielfach sehr wohlwollend aufgenommen und umgesetzt wurde. Ausführlich dazu Bieresborn, S. 100 ff..

${ }^{479}$ Müchel, S. 23

${ }^{480}$ Bieresborn, S. 122

${ }^{481} \mathrm{Im}$ 13. Jahrhundert wurde in Eilsachen auf das schriftliche Libell verzichtet und dem Kläger wurde gestattet, die Klage mündlich zu Protokoll zu geben. Briegleb, S. 64

${ }^{482}$ Bei dinglichen Klagen musste der historische Klagegrund spätestens im Beweisverfahren auch angeführt werden, da der Kläger sein Eigentum in der Regel nicht beweisen konnte, ohne den Erwerbsgrund zu nennen. Müchel, S. 27

${ }^{483}$ Müchel, S. 26

${ }^{484}$ H. Kaufmann, JZ 1964, S. 482, 484

${ }^{485}$ Müchel, S. 29

${ }^{486}$ Müchel, S. 32

${ }^{487}$ Bierseborn, S. 121
} 
on des Klägers ${ }^{488}$, die Form des Libells sowie die Frage, ob das richtige Gericht angerufen worden war. ${ }^{489}$ War das Libell fehlerhaft, wurde die Klage unverzüglich abgewiesen, ansonsten wurde das Libell zu den Akten genommen. ${ }^{490}$

\section{b. Ladung (citatio)}

Hatte der Kläger seine Handlungen ordnungsgemäß vorgenommen, erfolgte die Ladung des Beklagten durch das Gericht. Dabei wurde der Beklagte durch einen Gerichtsboten oder den Geistlichen des Ortes im Auftrag des Richters aufgefordert, an einem bestimmten Termin und $\mathrm{Ort}^{491} \mathrm{zu}$ erscheinen. Die schriftliche Ladung musste weiterhin die genauen Namen des Klägers und Beklagten ${ }^{492}$ sowie den Namen des Richters enthalten. ${ }^{493}$ Ebenso musste eine kurze Beschreibung des geltend gemachten Anspruchs enthalten $\operatorname{sein}^{494}$ oder dem Beklagten eine Kopie des Klaglibells übergeben werden. ${ }^{495}$

Über die Ladung wurde dem Richter Bericht erstattet und ein Protokoll angefertigt. ${ }^{496}$ War die persönliche Ladung nicht möglich, konnte subsidiär eine öffentliche Ladung erfolgen. ${ }^{497}$

Die erfolgte Ladung entwickelte verschiedene Rechtswirkungen. Erschien der Beklagte nicht, hatte er mit Säumnisfolgen zu rechnen. Ferner trat die Rechtshängigkeit ein, ${ }^{498}$ womit der Gerichtsstand festgelegt und das Streitobjekt verstrickt wurde. ${ }^{499} \mathrm{Ob}$ auch die Unterbrechung der Verjährung eintrat, ist umstritten. ${ }^{500}$

\section{c. Oblatio libelli}

War dem Beklagten das Libell nicht bereits mit der Ladung übergeben worden, geschah dies im ersten Termin vor dem Richter, sog. oblatio libelli. Dabei übergab der Kläger oder der Richter dem Beklagten das Libell ${ }^{501}$ und letzterer setzte eine Frist, innerhalb derer sich der Beklagte entscheiden musste, ob er anerkennen oder bestreiten wollte. ${ }^{502}$ Erst wenn der Beklagte das Libell angenommen hatte, konnte der Prozess stattfinden. ${ }^{503}$ Bevor der Beklagte das Libell jedoch annehmen musste, konnte er dilatorische und prozessuale Einreden geltend machen. ${ }^{504}$ Erst mit unbedingter Annahme des Libells war der Prozess endgültig eingeleitet.

\footnotetext{
${ }^{488}$ Ausführlich dazu: Baumgarten, S. 11 ff..

${ }^{489}$ Müchel, S. 41

${ }^{490}$ Dies geschah in der Regel durch Abschrift und Beifügung des Datums der Vorlage. Allerdings wurde das Libell nicht überall sofort schriftlich zu den Akten genommen, da eine Änderung grundsätzlich noch möglich war. Müchel, S. $41 \mathrm{f}$.

${ }^{491}$ Der Ort sollte nicht weiter als zwei Tagesreisen von der Grenze der Diözese entfernt sein, in der der Beklagte wohnte. Corpus Iuris Canonici X, 1, 3, 28

${ }^{492}$ Durantis, Lib. II, de citat. $\S 1$ Nr. 20

${ }^{493}$ Müchel, S. 45

${ }^{494}$ Müchel, S. 46

${ }^{495}$ Endemann, ZfdZ 15 (1891) S. 177, 199

${ }^{496}$ Müchel, S. 53

${ }^{497}$ Müchel, S. 55

${ }^{498}$ Müchel, S. 56; a.A. Endemann, ZfdZ 15 (1891) S. 177, 212

${ }^{499}$ Der Gegenstand durfte nicht mehr veräußert, abgetreten, verpfändet oder anderweitig verändert werden. Corpus Iuris Canonici X, 2, 16, 3: ut lite pendente nihil innovetur

${ }^{500}$ Für eine solche Wirkung Müchel, S. 66 und Kaser/Hackl, S. 635 (für den Rescriptenprozess); a.A. Endemann, ZfdZ 15 (1891) S. 177, 232 erst ab Litiskontestation.

${ }^{501}$ R. Schmidt, Klagänderung, S. 54

${ }^{502}$ Durantis, Lib. I de lib. obl.

${ }^{503}$ R. Schmidt, Klagänderung, S. 55

${ }^{504}$ So konnte das Nicht-Einhalten von Ladungsfristen gerügt oder Bedenken gegen die Person des Richters geltend gemacht werden. Endemann, ZfdZ 15 (1891), S. 177, 212
} 


\section{d. Weiterer Prozessverlauf}

Im weiteren Prozessverlauf wurden die Vorträge der Parteien ausgetauscht, wobei erst der Beklagte auf die Klage zu antworten hatte, bevor der Kläger seine Replik vorbringen durfte. Darauf konnten Duplik des Beklagten und Triplik des Klägers folgen ${ }^{505}$ Konnte der Beklagte keine prozessualen Einreden geltend machen ${ }^{506}$, erfolgte nach dem Austausch der Vorträge die Streitbefestigung (litis contestatio). War dies geschehen, ging der Prozess ins Hauptverfahren über. Die Klage durfte nicht mehr geändert werden, bezüglich der prozessualen dilatorischen Einreden trat Präklusionswirkung ein, und der Inhalt des Streites war unabänderlich fixiert. ${ }^{507}$

Vor der Beweiserhebung wurde mit Hilfe von positiones die Beweisbedürftigkeit von Behauptungen geklärt. In den positiones stellten die Parteien schriftlich einzelne Behauptungen auf. Dies konnte für dilatorische und prozessuale Einreden schon vor der litis contestatio geschehen. ${ }^{508}$ Der Gegner musste zu jedem Punkt Stellung beziehen. Tat er dies nicht oder bestritt er die Behauptung nicht, galt sie als zugestanden und der Beweis konnte entfallen. ${ }^{509}$ Erst wenn beide Parteien ihre positiones aufgestellt und auf die des Gegners geantwortet hatten, wurde über die verbliebenen streitigen Punkte Beweis erhoben. ${ }^{510}$

In diesem System manifestiert sich das Verhandlungsprinzip besonders deutlich, da ausschließlich die Parteien über den Streitstoff und die Beweisbedürftigkeit entschieden.

\section{Zusammenfassung}

Der römisch-kanonische Prozess ist von besonderer Bedeutung, weil er nicht nur das deutsche Verfahren entscheidend beeinflusste, sondern auch beim EquityVerfahren vor der Chancery Pate stand. ${ }^{511}$ Dabei sollte ein besonderes Augenmerk auf die Verfahrenseinleitung mittels Klaglibell gelegt werden sowie auf das System der positiones, in dem sich das Verhandlungsprinzip manifestiert. Beide Eigenarten wirkten in anderen Rechtsordnungen lange fort.

\footnotetext{
${ }^{505}$ Durantis, Lib. II, de except. et replic. $§ 5$

${ }^{506}$ Diese sollten die litis contestatio gerade verhindern. Corpus Iuris Canonici, X, 2, 28, 62

${ }^{507}$ Bieresborn, S. 135

${ }^{508}$ Endemann, ZfdZ 15 (1891) S. 177, 240 f., 252

${ }^{509}$ Durantis, Lib. II, de pos. § 2 Nr. 2

${ }^{510}$ Dazu Endemann, ZfdZ 15 (1891) S. 177, 252; R. Schmidt, Zivilprozess, S. 75 f.

${ }^{511}$ S. oben B.I.6.; Vgl. auch Wieaker, S. 105 Fn. 22 m.w.N.
} 


\section{Die Adaption des römisch-kanonischen Verfahrens in Deutschland und die Entwicklungen des gemeinrechtlichen Prozesses}

$\mathrm{Ab}$ dem 13. Jahrhundert kam es auf deutschem Gebiet zu einer Adaption des römisch-kanonischen Verfahrens.

\section{Die Adaption des römisch-kanonischen Verfahrens durch geistliche Gerichte}

Die ersten Gerichte, die nach dem römisch-kanonische Prozess verfuhren, waren geistliche Gerichte. ${ }^{512}$ Der Einfluss des Verfahrens auf die geistlichen Gerichte Deutschlands hatte verschiedene Gründe. Zum einen griffen päpstliche Legaten in das Verfahren ein. ${ }^{513}$ Gleiches geschah durch Delegationen, auf die der Papst Streitigkeiten übertrug, die im Rahmen der Appellation an ihn gelangt waren. ${ }^{514}$ Zum anderen wurde die Angleichung des Verfahrens an Rom durch das Rechtsstudium deutscher Geistlicher an italienischen Universitäten ${ }^{515}$ und die Verwendung von Formularbüchern als Leitfäden für die Durchführung eines Zivilprozesses $^{516}$ gefördert. Durch diese Einflüsse kam es bis zum Ende des 13. Jahrhunderts zu einer fließenden Rezeption des römisch-kanonischen Verfahrens auch auf unterster Ebene. ${ }^{517}$

Im 14. und 15. Jahrhundert gaben die Offizialstatuten das Verfahren vor den geistlichen Gerichten wieder. ${ }^{518}$ Diese wurden vom Bischof für sein Bistum erlassen und enthielten weitestgehend ${ }^{519}$ den römisch-kanonischen Prozess. ${ }^{520}$ Grundsätzlich musste ein schriftliches Klaglibell eingereicht werden ${ }^{521}$ und eine schriftliche Ladung erfolgen. ${ }^{522} \mathrm{Im}$ Allgemeinen fand jedoch ein mündliches Verfahren statt. Die einzelnen Schriftstücke mussten verlesen werden, um in den Prozess eingeführt zu werden. ${ }^{523}$ Von Feinheiten abgesehen, folgte der Prozess jedoch römischkanonischen Prinzipien.

\section{Die Adaption des römisch-kanonischen Verfahrens vor den weltlichen Gerichten}

Ab dem 14. Jahrhundert „erlagen“ auch die weltlichen Gerichte zunehmend dem Einfluss des römisch-kanonischen Verfahrens. Dies hatte verschiedene Gründe. Zum einen dehnten sich die Zuständigkeitsbereiche der geistlichen Gerichte immer weiter aus, da sie persönliche Jurisdiktion über alle Geistlichen und sogar deren Gesinde und Personal ausübten. ${ }^{524}$ Des weiteren konnten geistliche Gerichte angerufen werden, wenn dem Beklagten ein sündhaftes Vergehen vorgeworfen wurde. ${ }^{525} \mathrm{Um}$ dieses Vergehen zu prüfen, musste auch das zugrunde liegende

\footnotetext{
${ }^{512}$ Wieacker, S. 116 ff. spricht von einer uneigentlichen kanonistischen Frührezeption.

${ }^{513}$ Bieresborn, S. 169

${ }^{514}$ Ober, Archiv für katholisches Kirchenrecht 90, S. 599, 605

${ }^{515}$ Ober, Archiv für katholisches Kirchenrecht 90, S. 599, 610

${ }^{516}$ Ott, ZRG Kan. 3 (1913), S. 1, 44; Trusen, S. 130; vgl. Beipiele bei Muther, Geschichte, S. 2, 33, 54

${ }^{517}$ Ober, Archiv für katholisches Kirchenrecht 90 (1910), S. 599

${ }^{518}$ Boockmann, S. 129; Steins, ZRG Kan. 59 (1973), S. $191 \mathrm{f}$.

${ }^{519}$ Oft fanden sich lokale Eigenheiten minderer Bedeutung.

${ }^{520}$ Steins, ZRG Kan. 59 (1973), S. 191, 209

${ }^{521}$ Steins, ZRG Kan. 59 (1973), S. 191, 245

${ }^{522}$ Steins, ZRG Kan. 59 (1973), S. 191, 235 f.

${ }^{523}$ Steins, ZRG Kan. 59 (1973), S. 191, 223

${ }^{524}$ Trusen, S. 38

${ }^{525}$ Bieresborn, S. 189
} 
Rechtsverhältnis geklärt werden. ${ }^{526}$ Zum anderen waren die geistlichen Gerichte beim Volk wesentlich beliebter als die weltlichen und so wurde jede Möglichkeit genutzt, das Verfahren vor die geistlichen Gerichte zu bringen. ${ }^{527}$ Dies lag einmal am Verfahren vor den geistlichen Gerichten, das als fairer empfunden wurde, ${ }^{528}$ die Möglichkeit zur Vertretung gab, mit einem festen Instanzenzug Rechtssicherheit vermittelte und billiger war. ${ }^{529}$ Zusätzlich hatten die kirchlichen Gerichte bessere Vollstreckungsmöglichkeiten. ${ }^{530}$ Die Kirche hatte mit Kirchenbann, Interdikt und Exkommunikation Mittel zur Durchsetzung an der Hand, die weltlichen Gerichten nicht zur Verfügung standen. ${ }^{531}$

Dieser Entwicklung hin zu den geistlichen Gerichten versuchten die weltlichen Gerichtsherren entgegen zu wirken. Dabei konkurrierten die weltlichen Gerichte noch untereinander. Zum einen sprach das Reichshofgericht, später Reichskammergericht, unter der persönlichen Justizgewalt des Königs Recht. Zum anderen übten die Landesherren ihre Gerichtsbarkeit aus.

\section{a. Entwicklungen bei der Rechtsprechung des Königs}

Das erste weltliche Gericht, das nach dem römisch-kanonischen Prozess verfuhr, war das Reichshofgericht. Dies geschah bereits im 12. Jahrhundert, allerdings ausschließlich in Verfahren, in denen geistliche Angelegenheiten betroffen waren. ${ }^{532}$ Versuche, das römisch-kanonische Verfahren auch auf weltliche Streitigkeiten auszudehnen, scheiterten jedoch. ${ }^{533}$ Im Laufe der nächsten zwei Jahrhunderte verlor das Reichshofgericht zunehmend an Ansehen und Bedeutung, ${ }^{534}$ bis es Mitte des 15. Jahrhunderts ganz aufhörte zu existieren. ${ }^{535}$ Die persönliche Rechtsprechung des Königs wurde jedoch weiterhin ausgeübt. Dazu bediente sich der König des Instituts der Delegation, das heißt, er beauftragte, wenn er in einem Rechtsstreit angerufen wurde, Fürsten mit der Rechtsprechung. ${ }^{536} \mathrm{Da}$ es sich bei den Beauftragten häufig um geistliche Fürsten handelte, wurde das römisch-kanonische Verfahren auch in weltlichen Streitigkeiten angewandt. ${ }^{537}$

Parallel dazu entwickelte sich das königliche Kammergericht, in dem der König unter Beiziehung Gelehrter aus Hofkreisen seine persönliche Rechtsprechung ausübte. ${ }^{538} \mathrm{Da}$ es immer häufiger zu Kompetenzstreitigkeiten mit den geistlichen Gerichten kam, war der König auf die Unterstützung durch Rechtsgelehrte angewiesen, die zum größten Teil Kleriker waren. ${ }^{539}$ Unter dem Einfluss dieser geistlichen Juristen kam es zunehmend zur Anwendung römisch-kanonischen Verfahrensrechts, das deutsche Verfahrensprinzipien verdrängte. ${ }^{540}$ Aus dem königlichen Kammergericht entstand 1495 das Reichskammergericht als höchstes Gericht des

${ }^{526}$ Günther, S. 23

${ }^{527}$ E. Kaufmann, JuS 1961, S. 120; Muther, Römisches und kanonisches Recht, S. 29; Stobbe, II, S. 134

${ }^{528}$ Dies lag unter anderem an der Abschaffung des Gottesurteils zugunsten des Zeugenbeweises. Ott, ZRG Kan. 3 (1913), 1, 102 f.

${ }^{529}$ Trusen, S. 57

${ }^{530}$ Trusen, S. $58 \mathrm{f}$.

${ }^{531}$ Trusen, S. $58 \mathrm{f}$.

${ }^{532}$ Trusen, S. $170 \mathrm{f}$.

${ }^{533}$ Bieresborn, S. 200 f.

${ }^{534}$ Trusen, S. 178 f.; Urteile wurden sogar käuflich, vgl. dazu Schwartz, S. 69

${ }^{535}$ Schwartz, S. 68

${ }^{536}$ Vgl. Ficker, S. 360.

${ }^{537}$ Ficker, S. 360

${ }^{538}$ Trusen, S. 196

${ }^{539}$ Stölzel, I, S. 34 ff.

${ }^{540}$ Franklin, S. 45 f. 
Reiches, welches nicht mehr auf der persönlichen Rechtsprechung des Königs, sondern auf reichsständischer Grundlage fußte. ${ }^{54}$

Zur Vereinheitlichung des Verfahrens wurden im 16. Jahrhundert verschiedene Reichskammergerichtsordnungen bzw. Reichsabschiede erlassen, die das Verfahren vor dem Reichskammergericht regelten.

Schon nach den Reichskammergerichtsordnungen von 1495, 1500 und 1507 galt das ,gemaine“, also das rezipierte römische Recht, als Richtschnur, ${ }^{542}$ wenn auch noch mit großen Einfalltoren für lokales Recht und Gewohnheitsrecht. ${ }^{543}$ Das Verfahren war grundsätzlich mündlich, die Ladung erfolgte jedoch schriftlich, ${ }^{544}$ jeder mündliche Vortrag wurde schriftlich aufgezeichnet und Schriftsätze wurden zugelassen. ${ }^{545}$ Die Verfahrenseinleitung erfolgte mittels Klaglibell, das die Tatsachen enthalten musste, die zur Identifikation des klägerischen Anspruchs benötigt wurden. Ab 1500 erfolgte der Verfahrensablauf streng nach römisch-kanonischen Grundsätzen. Allerdings wurde die oblatio libelli abgeschafft und der Kläger hatte dem Beklagten die Klageschrift vor dem ersten Termin zuzusenden. ${ }^{546}$ Dabei blieb es ihm überlassen, ob er die Klage in Form eines gewöhnlichen Klaglibells oder in Positionen erhob. ${ }^{547}$ Ab 1507 musste das gesamte Tatsachenmaterial und alle Prozesshandlungen schriftlich eingebracht werden, damit das Gericht sie berücksichtigte. ${ }^{548}$ Die Freiheit, wahlweise mit einfachem Klaglibell oder in Positionen $\mathrm{zu}$ klagen, beseitigte die Kammergerichtsordnung von 1521, indem sie eine Artikulierungspflicht vorschrieb. ${ }^{549}$ Danach musste bereits die Klageschrift alle klagebegründenden Tatsachen in Positionen zergliedert enthalten. ${ }^{550}$ Das war nach dem römisch-kanonischen Prozess erst nach der Litiskontestation erforderlich gewesen. ${ }^{551}$ Auf dieses Vorbringen hatte der Beklagte einzeln zu antworten sowie seine Verteidigung ebenfalls artikuliert zu formulieren. Bestrittenes war vom Vortragenden zu beweisen, Unbestrittenes galt nach der Litiskontestation als zugestanden. ${ }^{52}$

Von grundlegender Bedeutung war, dass Kläger und Beklagter ihre Antwort auf das gegnerische Vorbringen und eigene Behauptungen kombiniert vorbringen mussten, um nicht präkludiert zu werden. ${ }^{553}$ Dies führte zu einer Veränderung des Verfahrens, weil nunmehr Behauptungs- und Beweisverfahren voneinander getrennt abliefen, da das Vorbringen beider Parteien abgeschlossen sein musste, bevor Beweis erhoben wurde. ${ }^{554}$ Diese Trennung war dem römisch-kanonischen Prozess fremd. Im römisch-kanonischen Verfahren wurde Beweis erhoben, sobald durch Bestreiten die Beweisbedürftigkeit festgestellt worden war. Das weitere Behauptungsverfahren zu anderen Punkten lief parallel dazu.

\footnotetext{
${ }^{541}$ Schwartz, S. 69

${ }^{542} \S 3$ der Kammergerichtsordnung von 1495

${ }^{543}$ Schwartz, S. 72

${ }^{544} \S 11$ der Kammergerichtsordnung von 1495

${ }^{545}$ Schwartz, S. $74 \mathrm{f}$.

${ }^{546}$ Tit. I $\S \S 1$ und 4 der Terminsordnung von 1508; R. Schmidt, Klagänderung, S. 96

${ }^{547}$ Tit. I $\S 7$ der Terminsordnung von 1508

${ }^{548}$ Bieresborn, S. 216 f.

${ }^{549}$ Tit. XIX § 5 der Kammergerichtsordnung von 1521

${ }^{550}$ Wetzell, S. 970

${ }^{551}$ Schwartz, S. 82

${ }^{552}$ Schwartz, S. 103

${ }^{553}$ Tit. XIX $\S 8$ der Kammergerichtsordnung von 1521; Schwartz, S. 82 f.

${ }^{554}$ Das System erinnert insoweit an das germanische Verfahren, wo Behauptungs- und Beweisverfahren durch das Beweisurteil getrennt waren.
} 
Der Artikelzwang wurde zwar 1523 vorerst abgeschafft, 1570 aber praktisch wieder eingeführt. ${ }^{555}$

Mit der Kammergerichtsordnung von 1555 wurde erstmals ein relativ umfassendes Zivilprozessgesetz erlassen. ${ }^{556}$ Danach beantragte der Kläger bei Gericht die Ladung des Beklagten. Wenn das Gericht sie bewilligte, wurde sie vom Gerichtsboten schriftlich ausgeführt. ${ }^{557}$ Die Ladung erfolgte in der Regel auf den 30. Tag nach ihrer Ausstellung ${ }^{558}$ und musste ,Sach und Klag“ so bestimmt enthalten, dass der Beklagte sich darauf einstellen konnte. ${ }^{559}$

Im ersten Termin übergab der Kläger dem Beklagten die Klageschrift, worauf dieser vier Wochen Zeit zur Antwort hatte. War die Klage unartikuliert abgegeben worden, hatte der Kläger weitere vier Wochen zur Artikulierung seiner Klage. ${ }^{560}$ Im Großen und Ganzen wurde das Verfahren im Verhältnis zur Kammergerichtsordnung von 1521 wieder gelockert. Mit dem Reichsabschied von 1570 fand eine erneute Verschärfung des Verfahrens statt, die 1594 wiederum etwas zurückgenommen wurde.

Im Wesentlichen entsprach das Verfahren vor dem Reichskammergericht dem römisch-kanonischen Verfahren, auch wenn es einige Eigenheiten entwickelt hatte. Die Ladung wurde bei Gericht beantragt und von Amts wegen ausgeführt. Die Klage musste schriftlich vorgetragen, alle weiteren Parteivorträge zumindest protokolliert werden. Der Kläger war praktisch gezwungen, in der Klageschrift nicht nur den Streitgegenstand bzw. Klagegrund anzugeben, sondern sein gesamtes Vorbringen in Artikel zergliedert zu substantiieren.

\section{b. Entwicklungen der landesherrlichen Gerichtsbarkeit}

Die unterschiedliche Entwicklung des Zivilprozesses in den Territorien und Städten führte zu einer Teilung des Reichsgebietes. In den süd- und westdeutschen Gebieten fand schon ab 1350 eine Übernahme des italienischen Zivilprozesses nach römisch-kanonischen Prinzipien statt ${ }^{561}$ der sich dann weitgehend parallel zum kammergerichtlichen Verfahren entwickelte. ${ }^{562}$ Anders stellte sich die Situation in den rechtselbischen Ländern dar. Die Adaption des römisch-kanonischen Verfahrens fand nur sehr zögerlich statt, was sich hauptsächlich auf die Tatsache zurückführen lässt, dass durch den Sachsenspiegel das lokale Recht weitgehend kodifiziert war und somit eine verhältnismäßig hohe Rechtssicherheit bestand. ${ }^{563}$ Allerdings fand bis zur Mitte des 16. Jahrhunderts auch in Sachsen ein schleichender Einbruch römisch-kanonischer Verfahrensgrundsätze an den Gerichten statt. Dies lag an den teils lückenhaften Gerichtsordnungen, die durch Gerichtspraxis und Rechtslehre geschlossen wurden. Dabei neigten insbesondere die auch an den Gerichten tätigen Rechtsgelehrten der Universitäten dem römisch-kanonischen Verfahren zu. ${ }^{564}$ Die dadurch entstehende Rechtsunsicherheit versuchte man ab 1572 durch eine einheitliche Gerichtsordnung zu beseitigen. ${ }^{565}$ Endgültig gelang dies allerdings erst 1622 mit einer einheitlichen umfassenden Gerichtsord-

\footnotetext{
${ }^{555} \S 88$ des Reichsabschieds von 1570 wonach der Kläger in Positionen klagen musste, um überhaupt in den Genuss der speziellen Beantwortung seiner Behauptungen zu kommen.

${ }^{556}$ Schwartz, S. 87

${ }^{557}$ Th. 3, Tit. XII $\S \S 1$ und 2 der Reichskammergerichtsordnung von 1555

${ }^{558}$ Th. 3, Tit. XII $\S 4$ der Reichskammergerichtsordnung von 1555

${ }^{559}$ Th. 3, Tit. XII $\S 1$ der Reichskammergerichtsordnung von 1555

${ }^{560}$ Th. 3, Tit. XIII $\S 5$ der Reichskammergerichtsordnung von 1555

${ }^{561}$ R. Schmidt, Zivilprozeß, S. 83

${ }^{562}$ Bieresborn, S. 240; zur Entwicklung in Bayern speziell und ausführlich Schlosser, S. $221 \mathrm{ff}$.

${ }^{563}$ Engelmann, S. 121; R. Schmidt, Zivilprozeß, S. 86

${ }^{564}$ Schwartz, S. 135
} 
nung, die die Materie von Grund auf regelte, anstatt, wie ihre Vorgänger, nur Lücken zu schließen. ${ }^{566}$

\section{Der Sächsische Prozess und der gemeinrechtliche Prozess nach dem jüngs- ten Reichsabschied}

Mit Einführung der sächsischen Gerichtsordnung von 1622 begann eine Entwicklung weg vom römisch-kanonischen Verfahren. Dabei zeichnet sich die Gerichtsordnung von 1622 insbesondere dadurch aus, dass sie eine umfassende Zivilprozessordnung eigenständiger Machart darstellte, die neben germanischen und römisch-kanonischen Prinzipien auch eigenständige Ansätze hervorbrachte. Unter dem Einfluss des sächsischen Rechts entstand mit dem jüngsten Reichsabschied 1654 der gemeinrechtliche Prozess, welcher auf Reichsebene das Verfahren in einer Art Mischform aus sächsischem Verfahren und dem Verfahren vor dem Reichskammergericht neu regelte. ${ }^{567}$

\section{a. Das Verfahren nach der sächsischen Gerichtsordnung von 1622}

Nach der Gerichtsordnung von 1622 hatte der Kläger bei Gericht ein schriftliches Klaglibell einzureichen. Der Beklagte wurde sodann unter Beifügung einer Abschrift der Klage vom Gericht geladen. ${ }^{568}$

Das Klaglibell musste das gesamte Tatsachenmaterial, das im ersten Termin vorgebracht werden sollte, sowie eine ausführliche Geschichtserzählung enthalten, die allerdings nicht in artikulierter Form abgegeben werden durfte. ${ }^{569}$ Mit Hilfe der genauen Geschichtserzählung sollte der Streitgegenstand festgelegt werden, da der Beklagte auf jede der klagebegründenden Tatsachen zu antworten hatte, damit sie nicht als zugestanden galten. Durch den einseitig geäußerten Widerspruch des Beklagten auf eine Ausführung des Klägers erfolgte die Litiskontestation, die anders als im römisch-kanonischen Verfahren und im Kammergerichtsprozess von 1555 nicht ausdrücklich erklärt werden musste. ${ }^{570}$ Am Ende des Klagebeantwortungsverfahrens, das ausschließlich der Feststellung des Tatsachenmaterials diente, erging das Beweisurteil, in dem entschieden wurde, wer für die streitigen Tatsachenbehauptungen Beweis zu erbringen hatte. ${ }^{571}$ Außer der Klage, die schriftlich einzureichen war, erfolgte das Parteivorbringen mündlich und wurde bei Gericht protokolliert. ${ }^{572}$

\section{b. Der gemeinrechtliche Prozess}

Nach dem 30-jährigen Krieg wurde auf Reichsebene das Gerichtsverfahren erneut reformiert. Dies geschah mit dem jüngsten Reichsabschied von 1654, der als das wichtigste Prozessgesetz des ersten deutschen Reiches ${ }^{573}$ erachtet werden muss. ${ }^{574}$ Der jüngste Reichsabschied kombinierte das römisch-kanonisch orientierte

\footnotetext{
${ }^{565}$ Constitutiones und Ordnungen, den Rechtlichen Process und was denen Gerichten mehr anhängig ist, belangende; dabei handelte es sich allerdings um keine vollständige Prozessrechtskodifikation, sondern eher um eine lückenfüllende Bestandsaufnahme, die oft auf bestehende Gerichtspraxis verwies. Sehe dazu Schwartz, S. 135 f.

${ }^{566}$ Schwartz, S. $145 \mathrm{f}$.

${ }^{567}$ R. Schmidt, Klageänderung, S. 125; Schwartz, S. 116 f.

${ }^{568}$ Tit. IV $\S 1$ der Gerichtsordnung von 1622 ; siehe. auch Schwartz, S. 146

${ }^{569}$ Tit. V $\S 1$ der Gerichtsordnung von 1622

${ }^{570}$ R. Schmidt, Zivilprozeß, S. 87

${ }^{571}$ Tit. XX der Gerichtsordnung von 1622; siehe auch Schwartz, S. 148

572 Bieresborn, S. 249

${ }^{573}$ Das Heilige Römische Reich deutscher Nation findet insofern keine Beachtung.

${ }^{574}$ Schwartz, S. 116
} 
Reichskammergerichtsverfahren mit dem sächsischen Prozess, ${ }^{575}$ der vom römisch-kanonischen Verfahren nur wenig übernommen hatte und weitgehend dem germanischen Recht folgte. ${ }^{576}$

Dem sächsischen Recht folgend, musste das Klaglibell nicht mehr in Artikeln verfasst werden, sondern musste die der Klage zugrundeliegenden Tatsachen schlüssig und vollständig enthalten. Dazu war die einfache Geschichtserzählung ausreichend. ${ }^{577}$ Anders als im sächsischen Prozess wurde das allgemeine Bestreiten des Klägervortrags durch den Beklagten zugelassen. ${ }^{578}$ Das Erfordernis der ausdrücklichen Litiskontestation wurde fallengelassen. ${ }^{579} \mathrm{Um}$ den Prozess weiter $\mathrm{zu}$ beschleunigen, waren die Parteien angehalten, mit der Klage, bzw. der Klageerwiderung auch das jeweilige Beweismaterial mit einzureichen. ${ }^{580}$ Obwohl es sich dabei nicht um eine zwingende Vorschrift handelte, war es für den Kläger von Vorteil, das Beweismaterial beizufügen, da der Beklagte sich dann in seiner Erwiderung auch über das vorliegende Beweismaterial erklären musste. ${ }^{51}$ Das Schriftlichkeitsprinzip wurde verschärft, so dass die Parteien jegliches Parteivorbringen schriftlich bei Gericht einzureichen hatten ${ }^{582}$ und eine mündliche Verhandlung in den meisten Fällen überflüssig wurde. ${ }^{583}$

Der Übergang zum reinen Schriftwechselverfahren zwischen den Parteien, bei dem das Gericht nach Aktenlage entschied, wurde zwar nicht vom jüngsten Reichsabschied, aber beim Verfahren vor dem Reichshofrat ${ }^{584}$ vollzogen. ${ }^{585}$

\section{Zusammenfassung}

Zwischen dem 13. und dem 17. Jahrhundert veränderte sich der Zivilprozess im deutschen Reich grundlegend. Ausgehend von den Missständen des germanischen Verfahrens hielt der römisch-kanonische Prozess Einzug in die Gerichte auf deutschem Reichsgebiet. Anfänglich geschah dies bei den geistlichen Gerichten, später waren aber auch die weltlichen Gerichte gezwungen, römisch-kanonische Verfahrensprinzipien zu adaptieren, um auch weiterhin mit den geistlichen Gerichten konkurrieren zu können. Seine größte Ausdehnung fand der römischkanonische Prozess mit der Reichskammergerichtsordnung von 1555, die nur noch wenige deutschrechtliche Prinzipien enthielt.

Ausgehend vom sächsischen Recht, das sich einer Adaption des römisch-kanonischen Prozesses widersetzte, wurde nach dem 30-jährigen Krieg das römischkanonische Verfahren wieder teilweise verdrängt und mit dem gemeinrechtlichen Prozess nach dem jüngsten Reichsabschied von 1654 eine Art Mischverfahren geschaffen.

Ein wichtiges Merkmal des gemeinrechtlichen Prozesses war die extreme Schriftlichkeit, die mündliche Verhandlungen weitgehend überflüssig machte. Weiterhin musste der Kläger seine Klage nicht mehr in Artikeln formulieren, sondern in einfacher und schlüssiger Geschichtserzählung die anspruchsbegründenden Tatsa-

\footnotetext{
${ }^{575}$ R. Schmidt, Klageänderung, S. 125

${ }^{576}$ R. Schmidt, Zivilprozess, S. 91

$577 \S 34$ des jüngsten Reichsabschieds von 1654

${ }^{578}$ Schwartz, S. $121 \mathrm{f}$.

${ }^{579}$ Schwartz, S. 118

${ }^{580}$ Dies galt insbesondere für Urkunden. $§ 35$ des jüngsten Reichsabschieds von 1654

${ }^{581} \S 39$ des jüngsten Reichsabschieds von 1654

${ }^{582} \S 94$ des jüngsten Reichsabschieds von 1654

${ }^{583}$ Schwartz, S. 124; Wetzell, S. 896 f.

${ }^{584}$ Der Reichshofrat hatte ebenfalls Jurisdiktionsgewalt auf Reichsebene und fungierte als persönliche Rechtsprechung des Königs. Vgl. Schwartz, S. 124.

${ }^{585}$ Tit. III $\S \S 22,24$ der Reichshofratsordnung von 1654
} 
chen vortragen. Neben diesem notwendigen Inhalt der Klageschrift war es ratsam, vorhandene Beweise bereits beim Einreichen der Klageschrift beizulegen.

\section{Rechtsvergleichende Betrachtung}

\section{a. Allgemeine Entwicklungen}

Sowohl das Verfahren in England als auch dasjenige in Deutschland entwickelte sich von den germanischen Grundsätzen weiter. Diese Entwicklungen waren aber weder zeitlich noch inhaltlich parallel. Beide Verfahren wurden allerdings durch eine übergeordnete königliche Rechtsprechung, die ihren eigenen Prozessgrundsätzen folgte, beeinflusst.

Erste Verfahrensänderungen durch die königliche Rechtsprechung finden sich in Deutschland zwar schon zu fränkischer Zeit ${ }^{586}$, ein tiefgreifender Wandel weg vom germanischen Verfahren ist aber erst im 14. Jahrhundert zu beobachten und beruht auf dem Einfluss des römisch-kanonischen Verfahrens. Seinen vorläufigen Höhepunkt erfuhr diese Entwicklung mit der Reichskammergerichtsordnung von 1555, die nur noch wenige deutschrechtliche Grundsätze enthielt.

In England entwickelte sich der Zivilprozess bereits im 12. und 13. Jahrhundert weiter und ließ die germanischen Prinzipien hinter sich. Dabei kam es aber nicht wie in Deutschland zu einer Adaption römischer Rechtsideen. Vielmehr entwickelte sich mit dem Writ-System ein eigenständiges Verfahren, das in seiner Grundstruktur speziellen Teilen des römischen Aktionenwesens erstaunlich ähnelt, ohne dass es gelungen wäre, tatsächliche Einflüsse des römischen auf das englische Recht nachzuweisen. Das Writ-System entstand durch die Zentralisierung der Gerichtsgewalt am Königshof in London.

Sowohl in Deutschland als auch in England kam es aber im Laufe der Zeit zu einer starken Modifikation des Verfahrens.

In Deutschland geschah dies durch den starken Einfluss des sächsischen Zivilprozesses, welcher erheblich mehr deutschrechtliche Prinzipien bewahrt hatte, als die Kammergerichtsordnung. Beide Verfahren verschmolzen im Folgenden zum gemeinrechtlichen Prozess, der als Mischform aus römisch-kanonischem und sächsischem Verfahren im Reich ${ }^{587}$ und in den Territorien galt.

In England entwickelte sich das Verfahren nach Common Law weitgehend unberührt von äußeren Einflüssen. Schon ab dem 13. Jahrhundert entstand als Folge der Ungerechtigkeiten, die sich aus dem starren Common Law-Verfahren ergaben, die Equity als eigenständige Billigkeitsrechtsprechung des Königs, bzw. der Chancery mit eigenständigem Verfahren. Common Law und Equity existierten in der Folgezeit parallel, ohne einander erheblich zu beeinflussen. Das Verfahren in Equity wurde allerdings bis ins 17. Jahrhundert vom römisch-kanonischen Prozess, der über den Court of Admiralty im englischen Recht Einzug gehalten hatte, beeinflusst. Zur gegenseitigen Beeinflussung der Verfahren kam es hingegen erst im 19. Jahrhundert, was in der Zusammenlegung der Gerichtszweige gipfelte.

\section{b. Entwicklungen im Common Law-Verfahren}

Im deutschen Recht wurde das Verfahren im 15. Jahrhundert dem römisch-kanonischen Prozess angepasst. Die Klage wurde per Klaglibell erhoben und der Beklagte im Anschluss geladen. Das Klaglibell musste anfänglich den Anspruch in tatsächlicher Hinsicht nur individualisieren, später substantiiert darstellen, dies

\footnotetext{
${ }^{586}$ Z.B. der Übergang bei der Ladung von der mannitio zur bannitio.

${ }^{587}$ Gem. dem Jüngsten Reichsabschied von 1654.
} 
zum Teil sogar in artikulierter Form. Erst unter sächsischem Einfluss wurde im gemeinrechtlichen Prozess die extreme Artikulierungspflicht wieder abgeschafft. Allerdings musste die Klageschrift weiterhin alle klagebegründenden Tatsachen schlüssig und vollständig enthalten.

Anders gestaltete sich die Entwicklung im Common Law. Dort erfolgte die Einleitung des Verfahrens mittels eines Writs, eines königlichen Befehls, den Anspruch zu begleichen oder vor Gericht zu erscheinen. Insoweit stellte das Writ gleichzeitig Klage und Ladung dar. Die entscheidende Besonderheit lag in der Tatsache, dass das Writ nicht die prozessuale Geltendmachung eines materiellrechtlichen Anspruchs darstellte, wie in Deutschland das Klaglibell. Im Common Law folgte der materiellrechtliche Anspruch der prozessualen Geltendmachung. D.h., ein Anspruch konnte nur eingeklagt werden, wenn es ein passendes Writ gab, unter dessen abstrakte Voraussetzungen sich die Tatsachen des konkreten Falles subsumieren ließen. Insofern enthielt das Writ zwar einen Tatsachenvortrag, aus diesem ergab sich aber nicht der Anspruch. Die Klage hatte nur Aussicht auf Erfolg, wenn die Tatsachen zum abstrakten Wortlaut des Writs passten. Dies führte verstärkt zu Problemen, als die Möglichkeit, neue Writs auszustellen, 1258 erheblich beschränkt wurde.

Der Tatsachenvortrag im Writ hatte somit nur für die prozessuale Durchsetzung des Anspruchs Bedeutung und informierte den Gegner darüber, um welchen Streit es ging. Die eigentliche Sachverhaltserörterung fand erst mit den Pleadings statt. Die Pleadings waren hochformalisierte und präzise Parteivorträge, die äußerlich in ihren Anforderungen an die artikulierten Vorträge im deutschen Prozess erinnern.

Diese Ähnlichkeit beschränkt sich jedoch auf Äußerlichkeiten, da Pleadings und Artikel unterschiedliche Ziele verfolgten. In den Pleadings klärten die Parteien untereinander, welche Tatsachen streitig waren und im Hauptverfahren einer Entscheidung zugeführt werden sollten. Insoweit war der Inhalt der Pleadings nicht Entscheidungsgrundlage für das Gericht, sondern allenfalls ihr Ergebnis. Die Artikel im deutschen Verfahren hingegen dienten der Entscheidung durch das Gericht, über welche Tatsachen Beweis erhoben werden musste, indem nach Abschluss der Parteivorträge festgestellt wurde, welche Punkte bestritten waren. So konnte das Vorbringen einer Partei im deutschen Verfahren auch gegen sie verwendet werden, da die Vorträge schon zur gerichtlichen Entscheidungsgrundlage wurden, wohingegen die Pleadings inhaltlich vom Gericht nicht zur Kenntnis genommen wurden; sie dienten ausschließlich der Information der Parteien.

Auf unterschiedliche Weise waren somit die Parteien Herren des Verfahrens. Sie entschieden, welche Tatsachen vorgetragen oder bestritten wurden. Für die Rechtsfindung hingegen war in beiden Ländern das Gericht zuständig. Die Beweiswürdigung hingegen nahmen im deutschen Verfahren Berufsrichter, im Common Law-Verfahren Geschworene vor. Die Verhandlungsmaxime galt genauso wie der Dispositionsgrundsatz in beiden Systemen. Allerdings blieb der englische Kläger an das Writ und das daraus folgende Verfahren gebunden. Stellte sich im Prozess heraus, dass der Anspruch mit Hilfe eines anderen Writs hätte geltend gemacht werden müssen, scheiterte die Klage unter dem erworbenen Writ.

Beide Verfahren neigten zu jener Zeit zu verstärkter Schriftlichkeit. Die Klageeinleitung geschah durch ein Schriftstück, wobei das Klaglibell schon den ersten Sachvortrag darstellte, das Writ hingegen lediglich bedingter Ladungsbefehl war. Der weitere Verfahrensablauf bewegte sich im Folgenden immer weiter vom Mündlichkeitsprinzip weg und zur Schriftlichkeit hin. Dies geschah in England 
insbesondere durch die Protokollierung des Parteivorbringens und die Einführung der schriftlichen Pleadings. Auch in Deutschland wurde die Einreichung von Schriftsätzen und die Protokollierung der mündlichen Vorträge die Regel. Im gemeinrechtlichen Prozess des 17. Jahrhunderts wurde das mündliche Verhandeln praktisch abgeschafft.

\section{c. Entwicklungen im Equity-Verfahren}

Die Entwicklung des Equity-Prozesses verlief nicht so isoliert von äußeren Einflüssen wie die des Common Law-Verfahrens. Vielmehr öffneten sich im Equity-Prozess Einfalltore, durch die kontinentale und insbesondere römisch-kanonische Einflüsse das Verfahren veränderten und prägten. Daher weist der Equity-Prozess auch größere Gemeinsamkeiten mit den deutschen Verfahren des 15.17. Jahrhunderts auf.

Beide Verfahren wurden mit Hilfe eines Schriftsatzes (Bill of complaint und Klaglibell) begonnen, in dem der klägerische Tatsachenvortrag in (relativ) freier Geschichtserzählung dargestellt wurde. Der Tatsachenvortrag war auch nicht wie beim Writ in genaue Worte zu fassen oder einem bestimmten Formular anzupassen. Die Geltendmachung eines Anspruches in equity entsprach somit eher dem kontinentaljuristischen, materiellrechtlich geprägtem System als dem aktionenrechtlichen Common Law. Worüber Beweis erhoben werden sollte, entschied die Reaktion des Beklagten. Nur über die bestrittenen Tatsachen wurde Beweis erhoben. Im 17. Jahrhundert kam es zu teils erheblichen Verfahrensänderungen. So hatte der Kläger die Beweismittel bereits in der Bill of complaint anzugeben. Weiterhin musste er konkrete Fragen (Interrogatories) an den Beklagten stellen, damit dieser zu qualifizierten Antworten auf konkrete Fragen gezwungen wurde. Diese Interrogatories hatten die gleichen Wurzeln wie die Artikel im deutschen Verfahren, da sie von den Positionen des römisch-kanonischen Verfahrens abgeleitet waren. Sie dienten dem Zweck, die streitigen Punkte schneller zu identifizieren.

Das Equity-Verfahren war dem deutschen Verfahren somit wesentlich ähnlicher als der Common Law-Prozess, zum einen, da es nicht aktionenrechtlich geprägt war, sondern einem Billigkeitsgedanken folgte, mithin dem materiellrechtlichen Verständnis ähnlicher war, zum anderen, da es wie das deutsche Recht römischkanonischen Einflüssen ausgesetzt war.

\section{d. Gesellschaftspolitische Gründe für die zivilprozessualen Reformen}

Gegen Ende des Mittelalters nahm der Humanismus starken Einfluss auf Europas Entwicklung. Ausgehend von den italienischen Universitäten entwickelten sich neue Vorstellungen von Wissenschaft, Gesellschaft und Staat.

Im Rahmen dieser Strömung schwappte auch der römisch-kanonische Prozess von Italien nach Deutschland über. Das Recht, Recht zu sprechen, gewann zunehmend an Bedeutung, was dazu führte, dass die verschiedenen Gerichte zu konkurrieren begannen. Dies geschah zum einen durch das Bereitstellen eines fairen Verfahrens, zum anderen, indem man versuchte, die Zuständigkeit anderer Gerichte zu beschneiden.

Je weiter sich Europa auf den Absolutismus zubewegte, desto stärker bemächtigte sich der Staat auch des Zivilprozesses. In Deutschland geschah dies durch zunehmende Schriftlichkeit und Mittelbarkeit des Verfahrens. Auch wurde das gesamte Verfahren in berufsrichterliche Hände gegeben und die Urteiler abge- 
schafft. ${ }^{588}$ Damit wurde das Recht aus den Händen des Volkes (in Person der Urteiler) genommen und zum Monopol des Staates gemacht. Ferner wurde mit den Urteilern eine Garantie gegen staatliche Willkür abgeschafft. ${ }^{589}$

Diese Entwicklung zeigte sich im gemeinrechtlichen wie im sächsischen Prozess. Die extreme Schriftlichkeit ${ }^{590}$ führte zu faktischer Nicht-Öffentlichkeit, sogar was die Parteiöffentlichkeit anbelangte, wodurch das Verfahren der Parteiherrschaft zunehmend entzogen wurde.

Diese Entwicklung hatte freilich nicht nur negative Folgen. Insbesondere Artikulierungszwang und die Aufgliederung in Positionen, die sich als zu schwerfällig erwiesen, führten zu einer Umstellung des Rechtsdenkens von der Rechtsfindung hin zur Rechtsanwendung durch eine neue rationale und differenzierte Methodik der Rechtswissenschaft. ${ }^{591}$

Die Entwicklung in England war dazu eher gegenläufig. Es kam zu keinem Konkurrenzkampf zwischen einzelnen Gerichten. Statt dessen nahm die Chancery die einheitliche Rechtspflege in die Hand, indem sie versuchte, die Mängel des Common Law zu korrigieren. Das Recht blieb jedoch im Wesentlichen in der Hand der Common Law-Gerichte, die keinen paternalistischen Anspruch entwickelten, sondern sich auf eine Art Schiedsrichterfunktion beschränkten. Die Urteilsfindung blieb in der Hand von Geschworenen. Verstärkte Schriftlichkeit betraf in erster Linie das Pleading-Verfahren, das ausschließlich von den Parteien betrieben wurde. Der Zugang zu den Gerichten und damit zum Recht hing davon ab, dass der Kläger selbst das richtige Writ auswählte. Die Verantwortung lag also ausschließlich bei den Parteien.

Etwas anderes gilt für die Equity. Diese war ja gerade aus einem Fürsorgegedanken entstanden, nämlich, dass der König für Gerechtigkeit zu sorgen habe. Insofern nahm sich der Staat dieses Verfahrens auch stärker an. Das erklärt auch, warum sich insoweit größere Ähnlichkeiten zum deutschen Verfahren finden.

\footnotetext{
${ }^{588} \mathrm{Vgl}$. Wieacker, S. 183

${ }^{589}$ a.a.O.

${ }^{590}$ Im gemeinrechtlichen Prozess handelte es sich sogar um einen Aktenprozess, bei dem der Richter nur den Akteninhalt wahrzunehmen hatte (quod non est in actis, non est in mundo).

${ }^{591}$ Vgl. dazu ausführlich Wieacker, S. 187 ff.
} 


\section{Die preußischen Reformversuche des 18. Jahrhunderts}

Bereits im 18. Jahrhundert wurde der gemeinrechtliche Prozess, der aus dem römisch-kanonischen und dem sächsischen Verfahren entstanden war, für dringend reformbedürftig gehalten.

\section{Verordnung über das Verfahren in Bagatellsachen von 1739}

Erstmalig brach die Verordnung über das Verfahren in Bagatellsachen von 1739 mit dem gemeinrechtlichen System. Anfänglich fielen unter dieses Gesetz alle Streitigkeiten mit einem Wert von unter 50 Thalern, was faktisch mehr als die Hälfte aller Streitigkeiten ausmachte. ${ }^{592}$ Nach dem neuen Verfahren wurden Bagatellsachen rein mündlich ohne Anwälte verhandelt. ${ }^{593}$ Dabei wurde der mündliche Vortrag bei Gericht protokolliert. ${ }^{594}$ Schon ein Jahr später wurde die Verordnung auf Druck der Anwaltschaft dahingehend geändert, dass sie nur noch bei Streitsachen unter zehn Talern Anwendung fand.

\section{Project des Codicis Fridericiani Marchici 1748}

Ein weiterer Reformversuch wurde acht Jahre später mit dem „Project des Codicis Fridericiani Marchici 1748“ veröffentlicht, wobei es sich aber auch eher um eine Variante des gemeinrechtlichen Prozesses handelte. ${ }^{595}$ Die Klage musste in der Regel schriftlich eingereicht werden, ${ }^{596}$ wobei allerdings , ein Bauer, oder anderer gemeiner Mann ${ }^{6597}$ die Klage auch bei Gericht zu Protokoll geben konnte. ${ }^{598}$ Nach Einreichung der Klage wurde dem Beklagten eine Abschrift zugestellt. ${ }^{599}$ Das weitere Verfahren verlief grundsätzlich mündlich und wurde protokolliert ${ }^{600}$, wobei eine anwaltliche Vertretung nur bei Abwesenheit der vertretenen Partei gestattet war. ${ }^{601}$ In schwierigen oder umfangreichen Verfahren stand es im Ermessen des Gerichts, das schriftliche Verfahren anzuordnen. ${ }^{602}$

Die einzige bedeutende Neuerung im Gegensatz zum gemeinrechtlichen Prozess war die Vorschaltung einer obligatorischen Güteverhandlung mit dem Ziel eines Vergleiches. ${ }^{603}$

\section{Preußische Prozeß-Ordnung 1781}

Da auch der Codex Fridericianus Marchicus 1748 nicht die erwünschten Veränderungen bei der Vereinfachung und Beschleunigung des Zivilverfahrens gebracht hatte, wurde bei der Neuordnung des preußischen Zivilrechts ab $1780^{604}$ auch der Zivilprozess neu geordnet. ${ }^{605}$

\footnotetext{
${ }^{592}$ Schwartz, S. $476 \mathrm{f}$.

${ }^{593}$ Schwartz, S. 476

${ }^{594} \S 1$ der Verordnung über das Verfahren in Bagatellsachen.

${ }^{595} \mathrm{Vgl}$. auch die bayerische Gerichtsordnung von 1753; Wieacker, S. 328

596 Tit. VI $\$ 3$ Cod.I.F.M.

${ }^{597}$ Tit. VI $\S 3$ Cod.I.F.M.

${ }^{598}$ Tit. VI $\S 3$ Cod.I.F.M.

${ }^{599}$ Tit. VIII $\S 2$ Cod.I.F.M.

${ }^{600}$ Tit. III Cod.I.F.M.

${ }^{601}$ Schwartz, S. 490

${ }^{602}$ Th. 3 Tit. I $\S \S 2$ und 3 Cod.I.F.M.

${ }^{603}$ Th. 4 Tit. VII $\$ 3$ Cod.I.F.M.

${ }^{604}$ Wieacker, S. 329

${ }^{605}$ Stobbe, II, S. 456
} 
Dazu wurde 1781 der Entwurf einer preußischen Prozeß-Ordnung veröffentlicht, aus dem später die Allgemeine Gerichtsordnung als Ideal des absolutistischen Prozesses werden sollte. ${ }^{606}$

Nach dieser preußischen Prozeß-Ordnung musste der Kläger die Klage anmelden, d.h. er musste bei der Gerichtsregistratur schriftlich oder zur Niederschrift seine Klage anzeigen. Diese Anmeldung musste den Namen, Stand und Charakter sowie die Anschrift des Klägers und des Beklagten enthalten, weiterhin das Objekt der Klage und das Begehren des Klägers und schließlich die Erklärung des Klägers darüber, ob er zur Verhandlung persönlich erscheine oder warum er dieses nicht tue. ${ }^{67}$ Das Gericht prüfte die eigene Zuständigkeit, ${ }^{608}$ und bejahendenfalls nahm ein richterlicher Beamter, der Assistenzrat, die Klage auf. ${ }^{609}$ Der Assistenzrat forschte daraufhin im persönlichen Gespräch mit dem Kläger den genauen Sachverhalt ${ }^{610}$ sowie dessen Meinung zu eventuellen Einwendungen des Beklag$\operatorname{ten}^{611}$ aus, stellte die zur Verfügung stehenden Beweismittel fest ${ }^{612}$ und erstellte ein Informationsprotokoll. ${ }^{613}$ Dieses Protokoll musste der Kläger unterschreiben, nachdem es ihm verlesen worden war. ${ }^{614}$ Hielt der Kläger danach an seiner Klage fest, ${ }^{615}$ wurde der „Hauptbericht über die Klage“ verfasst und bei Gericht ohne weitere Verzögerung eingereicht. ${ }^{616}$ Dieser Hauptbericht stellte die förmliche Klageschrift dar.

Darauf erfolgte die „Verordnung auf die Klage“, d.h. die Zustellung der Klage durch das Gericht ${ }^{617}$ an den Beklagten, mit dem Befehl, den Kläger innerhalb einer bestimmten Frist zu befriedigen oder sich einem Assistenzrat gegenüber zu erklären, dass er sich auf den Prozess einlasse. ${ }^{618}$

Die Klageerwiderung durch den Beklagten erfolgte nach dem gleichen Muster wie das Erstellen der Klage durch den Kläger. ${ }^{619}$

Nicht nur bei der Erstellung von Klage und Erwiderung kamen dem Gericht große Ausforschungskompetenzen zu, sondern auch im anschließenden Verfahren. Die Verhandlungsmaxime galt zwar grundsätzlich fort, da der Richter nicht über das Vorbringen oder die Bereitschaft der Parteien zu bestreiten oder zuzugestehen hinwegschreiten konnte, aber das Gericht hatte das Recht und die Pflicht die Wahrheit von Amts wegen herauszufinden. ${ }^{620}$ Trotz dieser weiten Kompetenzen war die Verhandlungsmaxime aber nicht gänzlich aufgehoben, da über Unbestrittenes kein Beweis zu erheben war. ${ }^{621}$

\footnotetext{
${ }^{606}$ Wieacker, S. 329

${ }^{607}$ Th. 1 Tit. II $\S 4$ Pr. Proz.O.

${ }^{608}$ Th. 1 Tit. II $\S 10$ Pr. Proz.O.

${ }^{609}$ Th. 1 Tit. II $\S 11$ Pr. Proz.O.

${ }^{610}$ Th. 1 Tit. III $\S 4$ Pr. Proz.O.

${ }^{611}$ Th. 1 Tit. III $\S 6$ Pr. Proz.O.

${ }^{612}$ Th. 1 Tit. III $\S 5$ Pr. Proz.O.

${ }^{613}$ Th. 1 Tit. III $\$ 1$ Pr. Proz.O.

${ }^{614}$ Th. 1 Tit. III $\S 8$ Pr. Proz.O.

${ }^{615}$ War der Assistenzrat der Auffassung, die Klage sei unbegründet, konnte er dies dem Gericht anzeigen, und das Gericht konnte die Klage abweisen. Th. 1 Tit. III $\S 12$ Pr. Proz.O.

${ }^{616}$ Th. 1 Tit. III $\S 13$ Pr. Proz.O.

${ }^{617}$ Th. 1 Tit. V $\S 8$ Pr. Proz.O.

${ }^{618}$ Th. 1 Tit. IV Pr. Proz.O.

${ }^{619}$ R. Schmidt, Zivilprozeß, S. 96

${ }^{620}$ Vorbericht zum Corpus Iuris Fridericianum III, zitiert bei Conrad, II, S. 468

${ }^{621}$ Schwartz, S. 503
} 


\section{Allgemeine Preußische Gerichtsordnung 1793}

Mit der Allgemeinen Preußischen Gerichtsordnung (A.G.O.) wurde 1793 eine überarbeitete Version des Prozessrechts erlassen.

Die Klageeinleitung folgte den gleichen Grundsätzen, wie sie schon in der preußischen Prozeß-Ordnung niedergelegt worden waren; allerdings wurden die Assistenzräte zugunsten von Justizkommissaren abgeschafft, die die Parteien nunmehr frei wählen konnten. ${ }^{622}$ Trotzdem handelte es sich dabei nicht um Anwälte der Parteien, sondern um Beamte, die einer starken Kontrolle durch das Gericht ausgesetzt waren. ${ }^{623}$

Im Rahmen der Klageanmeldung wurde das Verfahren insofern vereinfacht, als dass eine schriftlich eingereichte Klageanmeldung, die inhaltlich allen Voraussetzungen einer förmlichen Klage entsprach und durch einen Justizkommissar eingereicht wurde, sogleich dem Beklagten zugestellt werden konnte. ${ }^{624}$ Gleiches galt für die Erwiderung der Klage. ${ }^{625}$

Obwohl auch die A.G.O. die Ermittlung der Wahrheit durch den Richter in den Vordergrund stellte, schaffte sie die Verhandlungsmaxime nicht $a b^{626}$ Die Verpflichtung des Richters zur Erforschung der Wahrheit betraf allein den streitigen Sachverhalt, wobei der Richter allerdings freie Hand hatte. ${ }^{62}$

\section{Zusammenfassung}

Die preußischen Reformen des 18. Jahrhunderts brachten eine Abwendung vom Prinzip der Schriftlichkeit, hin zum mündlichen Vortrag, der dann allerdings protokolliert wurde, um in dieser Form zur Grundlage der gerichtlichen Entscheidung zu werden. Die Klage musste jedoch weiterhin schriftlich oder zur Niederschrift eingereicht werden. Weiterhin wurden die anwaltlichen Befugnisse beschränkt, da der Gedanke herrschte, die rechtliche Betreuung des Bürgers sei Sache des Staates. Damit ging eine erhebliche Verstärkung des Amtsermittlungsgrundsatzes Hand in Hand, die aber nicht gänzlich zur Aufhebung der Verhandlungsmaxime führte.

\section{Rechtsvergleichende Betrachtung}

Im 18. Jahrhundert wurde in beiden Rechtssystemen versucht, den schleppenden Verfahrensablauf $\mathrm{zu}$ beschleunigen. Der gemeinrechtliche Prozess galt als bürgerfremd und undurchsichtig, das Common Law-Verfahren wurde als zu formstreng angesehen und das Risiko, das falsche Writ zu erwirken, bestand nach wie vor. Der Equity-Prozess wiederum war langwierig.

Um das Verfahren zu beschleunigen und zu modernisieren, schufen die Gesetzgeber Reformen, die allerdings nur bedingt in die gleiche Richtung gingen. Das englische Verfahren existierte schon länger relativ unverändert als der gemeinrechtliche Prozess, der sich in den deutschen Gebieten erst im 17. Jahrhundert durchgesetzt hatte. Daher ist zu verzeichnen, dass in England schon im 17. Jahrhundert Reformbemühungen bestanden, die die gleichen Missstände zu bekämpfen versuchten, wie dies in Preußen erst im 18. Jahrhundert der Fall war.

In Preußen war der Wohlfahrtsstaat auf dem Höhepunkt seiner Entwicklung angekommen. Es hatte sich ein Staatsverständnis entwickelt, das den Bürger als un-

\footnotetext{
${ }^{622}$ R. Schmidt, Zivilprozeß, S. 96 f.

${ }^{623}$ Schwartz, S. 511

${ }^{624}$ Th. 1 Tit. IV $\S 23$ A.G.O.

${ }^{625}$ Th. 1 Tit. IX $\S 15$ A.G.O.

${ }^{626}$ Einleitung zur A.G.O. $\$ \S 13$ und 14

${ }^{627}$ Schwartz, S. 516
} 
mündig betrachtete, und in diesem Geiste entstand auch die A.G.O., „die kennzeichnend für das absolutistische Prozessideal wurde". ${ }^{628}$

Daher entzog man den Zivilprozess weitestgehend der Herrschaft der Parteien; so wurde das Verhandlungsprinzip erheblich eingeschränkt und die Pflichten und Befugnisse des Gerichts, die Wahrheit zu erforschen, ausgedehnt. ${ }^{629}$ Im gleichen Maße wurde die Pflicht zum vollständigen Vortrag in der Klageschrift abgeschwächt, da der Richter dafür Sorge zu tragen hatte, dass der Sachverhalt umfassend ausgeforscht wurde. Dies spiegelt das Misstrauen in staatsbürgerliche Selbstverantwortung und den Glauben, die Ordnung für die Gesellschaft in einem starken Staat gefunden zu haben, wider. ${ }^{630}$

Das Common Law schlug eine andere Richtung ein. Man versuchte, die Langwierigkeit des Verfahrens zu entschärfen, indem man das versuchsweise Vorbringen (tentative Pleadings) verbot. Dadurch waren die Parteien gezwungen, schon im ersten Vortrag möglichst alle Tatsachen darzulegen. Taten sie dies nicht, liefen sie Gefahr, dass ihr späteres Vorbringen eine Abweichung vom bisherigen Vortrag darstellte und nicht mehr zugelassen wurde.

Reformen im Equity-Prozess waren größtenteils schon im 16. und 17. Jahrhundert vorgenommen worden. Insbesondere die Einführung der Interrogatories und die Pflicht, Beweismittel schon in der Bill of complaint anzuführen, seien hier erwähnt. ${ }^{631}$

Da sich das Staatsverständnis im England des 17. und 18. Jahrhunderts nicht gravierend geändert hatte, waren auch keine radikalen Änderungen vorgenommen worden. Mit wachsender Kolonial- und Handelsmacht wurden auch Zivilprozesse wichtiger, am System an sich wurde aber nicht gerüttelt. Der Staat stellte nur das Spielfeld und den Schiedsrichter und versuchte die Fairness des Verfahrens durch kleine Änderungen zu erhöhen. Das Spiel blieb, anders als in Preußen, das Gleiche.

In beiden Ländern waren die Reformen jedoch ineffektiv und weitgehend ohne Erfolg. Dies und politische wie wirtschaftliche Revolutionen führten dazu, dass sowohl in England als auch in Deutschland in der Folgezeit eine vergleichsweise radikale Umgestaltung des Prozessrechts angestrebt wurde.

\footnotetext{
${ }^{628}$ Wieacker, S. 329

${ }^{629}$ Wassermann, S. 33

${ }^{630}$ „Alles für das Volk, aber nichts durch das Volk.“ Vgl. Wassermann, S. 34.

${ }^{631}$ Siehe dazu oben B.I.3.b.bb.
} 


\section{Die Vorgänger der ZPO}

Am Anfang des 19. Jahrhunderts führte die Unzufriedenheit mit dem Zivilprozess $\mathrm{zu}$ einer Flut von Reformen in den Prozessordnungen der deutschen Partikularrechte, die zum Teil stark vom französischen Prozessrecht geprägt waren. ${ }^{632}$

Das politisch aufsteigende Bürgertum war mit dem schriftlichen gemeinen Prozess genauso unzufrieden wie mit dem autoritären preußischen. ${ }^{633}$

Insbesondere die west- und süddeutschen Länder orientierten sich daher am französischen Prozessrecht und betrieben die Stärkung der Mündlichkeit, ${ }^{634}$ Unmittelbarkeit, Konzentration und Parteidisposition. ${ }^{635}$

Auch Preußen konnte sich diesen Strömungen nicht vollständig widersetzen, und so wurde 1833 die Verordnung über den Mandats-, summarischen und Bagatellproceß erlassen, die die A.G.O. von 1793 zwar nicht grundlegend änderte, aber die Mündlichkeit des Verfahrens stärkte. Die mündliche Verhandlung wurde protokolliert, musste aber nicht zwingend durchgeführt werden. ${ }^{636}$ Die Einführung der obligatorischen mündlichen Verhandlung geschah in Preußen erst mit der Verordnung über das Verfahren in Civilprocessen von 1846. ${ }^{637}$ Bei der Einreichung der Klage wurden jedoch keine Änderungen zur A.G.O. von 1793 vorgenommen.

\section{Der Weg zu einer einheitlichen Prozessordnung}

Bemühungen um eine einheitliche Prozessordnung oder zumindest um einheitliche Grundsätze der Prozessordnungen der Länder kamen allerdings erst in der zweiten Hälfte des 19. Jahrhunderts auf.

Der Geist der Revolution von 1848 fand auch Niederschlag im Zivilprozess, den man als Garantie des Rechtsstaates empfand. Daher war im Rahmen der Reichsverfassung von 1848 die Mündlichkeit und Öffentlichkeit des Verfahrens festgelegt worden ${ }^{638}$ - Grundsätze, zu deren Umsetzung die Länder verpflichtet waren. ${ }^{639}$

\section{a. Die Bürgerliche Proceßordnung für das Königreich Hannover von 1850 (B.P.O)}

Die möglicherweise wichtigste Gesetzgebungsaktivität stellte die Hannoversche Prozessordnung von 1850 dar. ${ }^{640}$

Nach der B.P.O. musste die Klage schriftlich eingereicht werden. Neben den allgemeinen Anforderungen an Schriftsätze, wie die Bezeichnung der Parteien und deren Adressen, $\S 93$ B.P.O., legte $\S 187$ B.P.O. für die Klage besondere Voraussetzungen fest:

Die Klageschrift musste eine ,gedrängte, deutliche und zusammenhängende Darstellung der Thatsachen, worauf der geltend gemachte Anspruch sowohl in der Hauptsache als in etwaigen Nebenpuncten rechtlich beruht“, enthalten sowie „die

\footnotetext{
${ }^{632}$ Umfassend dazu Schwartz, S. $531 \mathrm{ff}$.

${ }^{633}$ Wieacker, S. 465; vgl. zu den naturrechtlichen Einflüssen auf den Zivilprozess Vossius, S. 26 ff.

${ }^{634}$ Vgl. § 216 der Badischen Prozeßordnung von 1831; Th. I § 1der Verordnung vom 22.07.1819,

Bayerisches Gesetzblatt Nr. VII. und Novelle für beschleunigte Verfahren von 1834; $§ 70$ des

Edikts von 1818 für das Königreich Württemberg und $\S 12$ der Novelle von 1822.

${ }^{635}$ Wieacker, S. 465

${ }^{636} \S 20$ der Verordnung von 1833

${ }^{637} \S \S 11,12$ der Verordnung von 1833

${ }^{638} \S 178$ der Reichsverfassung von 1848: „Das Gerichtsverfahren soll öffentlich und mündlich sein. “

${ }^{639}$ Art. 3 Ziffer 8 des Einführungsgesetzes; vgl. auch Kip, S. 38.

${ }^{640}$ Mittermaier, AcP 45 (1862), S. 99, 108 bezeichnet sie als ,wichtigste[n] Wendepunkt in der deutschen Gesetzgebung“.
} 
besonderen Thatsachen, aus welchen [der Anspruch] gerade diesem Kläger gegen diesen Beklagten zusteht, so wie eine genaue Bezeichnung des gesonderten Gegenstandes oder der begehrten Leistung bei Unterlassen“, § 187 Nr. 1 B.P.O. Weiterhin hatte der Kläger „ein bestimmtes Gesuch sowohl in Betreff der Hauptsache als der Nebenpunkte“ und den „den Kläger vertretenden [Anwalt]“ zu benennen, $\S 187$ Nr. 2 und 3 B.P.O. Schließlich gehörte in die Klageschrift noch „die Aufforderung des Beklagten, an dem vom Vorsitzenden des Gerichts festgesetzten Gerichtstage, und zwar vertreten durch einen bei dem Proceßgerichte angestellten Anwalt vor diesem zu erscheinen“, $§ 187$ Nr. 4 B.P.O. ${ }^{641}$

Erfüllte die Klageschrift die formalen Voraussetzungen, setzte das Gericht ohne weitere Schlüssigkeitsprüfung ${ }^{642}$ einen Termin zur mündlichen Verhandlung fest, $\S 187$ B.P.O. Der Beklagte wurde daraufhin durch Gerichtsbeamte geladen, die auch die Vermittlung der Schriftsätze zwischen den Parteien übernahmen, $\S \S 118$ ff. B.P.O.

Das Gericht war somit weder mit den Handlungen zur Vorbereitung der Klage noch mit dem anwaltlichen Schriftverkehr befasst. Dies ergibt sich aus dem strengen Mündlichkeitsprinzip, das vorsah, dass das Gericht einzig aufgrund der mündlichen Verhandlung zu entscheiden hatte, § 101 B.P.O. Sowohl Klage als auch Schriftverkehr zwischen den Parteien hatten demnach nur die Funktion, die mündliche Verhandlung unverbindlich vorzubereiten ${ }^{643}$ Mit dem Umschwung zur Mündlichkeit wurde die Einheit der mündlichen Verhandlung eingeführt, selbst wenn die Verhandlung in mehrere Termine zerfiel.

Anders als im preußischen Verfahren hatte der Richter keine weitreichenden Inquisitionsbefugnisse, sondern neben seinen verfahrensleitenden Aufgaben nur ein eingeschränktes Fragerecht, § 111 B.P.O. Das Betreiben des Prozesses lag allein in der Hand der Parteien. ${ }^{644}$

\section{b. Der Entwurf einer allgemeinen Gerichtsordnung für Deutschland (Hanno- verscher Entwurf)}

1862 wurde eine in Hannover tagende Kommission der Staaten des Deutschen Bundes eingesetzt, die einen Entwurf für eine gemeinsame Prozessordnung erarbeitete. ${ }^{645}$ In erster Lesung wurde 1864 der Entwurf vorgestellt, der sich stark an der B.P.O. von 1850 orientierte.

Der B.P.O. wurde die Mündlichkeit des Verfahrens als Regelfall entnommen. Schriftliche Verfahren waren nur in komplizierten Fällen vorgesehen. Auch war wie nach der B.P.O. die Klage schriftlich einzureichen, das Verhandlungsprinzip galt, und die Parteien mussten anwaltlich vertreten sein. ${ }^{646}$ Anders als nach der B.P.O. war nach dem Hannoverschen Entwurf ein schriftliches Vorverfahren zur Entlastung des Gerichts vorgesehen, bei dem die Parteien unter Überwachung des Gerichts per Schriftsatzaustausch die Verhandlung vorbereiteten. ${ }^{647}$ Ein weiterer erheblicher Unterschied zur B.P.O. stellte die Einschränkung des Verhandlungsprinzips dar. Nach $\S 139$ des Entwurfs konnte das Gericht unbestrittene Tatsa-

\footnotetext{
${ }^{641}$ Gem. $§ 67$ bestand Anwaltszwang, es sei denn, die Partei hatte ein juristisches Examen bestanden und wohnte am Gerichtsort.

${ }^{642}$ Leonhardt, S. $51 \mathrm{f}$.

${ }^{643}$ Dahlmanns, S. 38

${ }^{644}$ Diese konnten sogar vereinbaren, gesetzliche Fristen zu verlängern, $§ 1152-154$ B.P.O.

${ }^{645}$ Hellweg, AcP 61 (1878), S. 78, 83

${ }^{646}$ Schwartz, S. 623

${ }^{647} \S \S 236$ ff. der ersten Lesung
} 
chenvorträge als zugestanden erachten. Dabei wurde ihm allerdings ein Ermessen eingeräumt. ${ }^{648}$

Die zweite Lesung des Entwurfs wurde im Frühjahr 1866 beendet und brachte im Gegensatz zur ersten Lesung nur wenige Änderungen. Auch danach war das Verfahren prinzipiell mündlich und öffentlich. Das Verhandlungsprinzip wurde hingegen wieder gestärkt, indem man das Ermessen des Gerichts abschaffte und nicht bestrittenen Tatsachen als zugestanden erachtete, soweit nicht aus dem übrigen Vortrag der Partei die Absicht zu bestreiten hervorging. ${ }^{649}$ Auch beim Einleitungsverfahren orientierte man sich stärker an der B.P.O. und schaffte das obligatorische schriftliche Vorverfahren ab. Stattdessen konnte das Gericht schon nach Einreichung der Klageschrift Termin zur mündlichen Verhandlung bestimmen. ${ }^{650}$ Ein schriftliches Vorverfahren konnte nur auf Antrag im ersten Verhandlungstermin angeordnet werden. ${ }^{651}$

\section{c. Prozessordnungen der Länder}

Während die Kommission in Hannover an ihrem Entwurf arbeitete, erließen diverse Staaten des deutschen Bundes eigene Prozessordnungen.

\section{aa. Badische Processordnung von 1864}

So wurde in Baden 1864 im Rahmen der Änderung der Gerichtsverfassung im Zivilprozess die Mündlichkeit als Grundprinzip eingeführt ${ }^{652}$, wobei von den Schriftsätzen abweichende Vorträge sowie Fragen des Richters und Antworten der Parteien protokolliert wurden. ${ }^{653}$ Ansonsten orientierte sich auch die Badische Processordnung von 1864 stark an der B.P.O. von 1850. ${ }^{654}$ Ein erheblicher Unterschied bestand jedoch bei der Klageeinleitung. Im Gegensatz zur B.P.O. unterzog das Gericht in Baden die Klage einer Zuständigkeits- und Schlüssigkeitsprüfung, bevor es die Klageschrift zustellte. ${ }^{655}$

\section{bb. Württembergische Civilprocessordnung von 1868}

Die Württembergische Civilprocessordnung von 1868 übernahm auch weitgehend das Verfahren, das in Hannover üblich war. Allerdings bestand ein Protokollierungszwang, soweit in der mündlichen Verhandlung von den Schriftsätzen abgewichen wurde ${ }^{656}$, die Parteien mussten sich nicht anwaltlich vertreten lassen und dem Gericht wurden stärkere prozessleitende Kompetenzen eingeräumt. ${ }^{657}$ Auch das schriftliche Vorverfahren erfolgte unter vermittelnder Einbeziehung des Gerichts. ${ }^{658}$ Anders als in Baden wurde in Württemberg die Klage nur auf ihre formellen Voraussetzungen geprüft, nicht auf ihre Schlüssigkeit. ${ }^{659}$

\footnotetext{
${ }^{648}$ Vg. auch Schwartz, S. 626.

${ }^{649}$ \& 129 der zweiten Lesung

${ }^{650}$ S. $\S \S 176,231,233$ der zweiten Lesung

${ }^{651} \S 239$ der zweiten Lesung

${ }^{652} § 993$ der Badischen Processordnung von 1864

${ }^{653} \S \S 278$ sowie 1002-1006 der Badischen Processordnung von 1864

${ }^{654}$ Kip, S. $48 \mathrm{f}$.

${ }^{655} \S 1015$ i.V.m. $\S \S 250,262$ der Badischen Processordnung von 1864

${ }^{656}$ Art. 195 der Württembergischen Civilprocessordnung; vgl. auch Kip, S. 49

${ }^{657}$ Schwartz, S. 634

${ }^{658}$ Art. 333 ff. der Württembergischen Civilprocessordnung

${ }^{659}$ Art. 322 der Württembergischen Civilprocessordnung
} 


\section{cc. Entwurf einer Process-Ordnung in bürgerlichen Rechtsstreitigkeiten für den Preußischen Staat von 1864}

In Preußen wurde 1864 ein Entwurf veröffentlicht, der das Verfahren, wie es seit 1793 mit den Änderungen von 1833 und 1846 bestand, reformieren sollte. Dazu wurde die Mündlichkeit in allen Verfahren festgeschrieben, allerdings nicht mit letzter Konsequenz, wie dies in Hannover geschehen war. Die mündliche Verhandlung war zwar Grundlage der Entscheidung, jedoch durften Anträge und deren Begründungen, die ausschließlich mündlich gestellt worden waren, nicht berücksichtigt werden. ${ }^{60}$ Die Anwälte der Parteien betrieben das Verfahren anfänglich ohne Kenntnis des Gerichts. Zur Verhandlung kam es erst, wenn eine Partei dies beantragte ${ }^{661}$ und die Anträge überreicht wurden. Erst damit wurde die Sache bei Gericht anhängig. ${ }^{62}$

\section{d. Der Entwurf einer Civilproceßordnung für den Norddeutschen Bund}

Die Bemühungen um eine einheitliche Prozessordnung für den Norddeutschen Bund wurden 1867 wieder aufgenommen und führten $1870 \mathrm{zu}$ einem Entwurf einer Zivilprozessordnung, der einen Kompromiss zwischen dem hannoverschen Entwurf von 1866 und dem preußischen Entwurf von 1864 darstellte. ${ }^{663}$

Allerdings wurde der Entwurf nicht mehr in geltendes Recht umgesetzt, da 1870 der deutsch-französische Krieg begann, auf den die Einigung Deutschlands folgte.

\section{Rechtsvergleichende Betrachtung}

Im englischen wie im deutschen Recht wurden im 19. Jahrhundert durch umfassende Reformen die Grundlagen für das Verfahrensrecht der Gegenwart gelegt. Der Reformbedarf wurde in beiden Systemen schon im ausgehenden 18. Jahrhundert empfunden.

In England führte insbesondere die industrielle Revolution zu einem veränderten Gesellschaftsbild, wodurch Reformen im trägen Zivilprozess notwendig wurden. Politisch blieb England jedoch relativ unberührt von den Revolutionen auf dem Festland (Politik der splendid isolation).

In Deutschland setzte sich der „Bund der bürgerlichen Gesellschaft mit dem Nationalstaat ${ }^{6664}$ durch und führte dazu, dass die Vereinheitlichung des Rechts und der bessere Zugang zum Recht vorangetrieben wurde. ${ }^{665}$

Zur radikalen Erneuerung des Systems kam es jedoch in beiden Ländern erst gegen Ende des 19. Jahrhunderts. ${ }^{66}$ Der Weg zu diesen Reformen war gesäumt von Versuchen, das System behutsam gegen die bestehenden Widerstände zu ändern. Dabei begegneten die Reformbestrebungen in beiden Ländern unterschiedlichen Problemen, die jeweils aus einer starken Zersplitterung resultierten. In England bestand eine Rechtsspaltung durch Common Law und Equity. Es war frühzeitig erkannt worden, dass sich viele Probleme, wie zum Beispiel fehlende materielle Gerechtigkeit der Entscheidungen und lange Verfahrensdauer, lösen ließen, wenn man Common Law und Equity zu einem Verfahren verschmelzen würde. Allerdings wurde diese Ansicht von vielen Praktikern nicht geteilt, die versuchten, das bestehende System zu erhalten. Aufgrund der Rechtstradition in

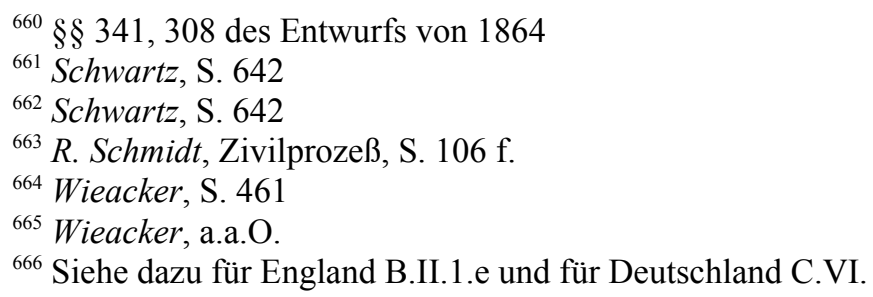


England, die der Rechtsprechung im Verhältnis zur Legislative erheblich mehr Einfluss auf das Recht zubilligte, als auf dem Kontinent, stellte es sich als schwierig dar, diese Widerstände zu brechen. So kam es zu kleinen Reformen, mit denen versucht wurde, das System ,schleichend“ zu ändern.

Die deutschen Reformbestrebungen kämpften gegen ein anderes Problem an, nämlich das der territorialen Zersplitterung. Da es auf deutschem Gebiet keine einheitliche Legislative gab, ließ sich die Vereinheitlichung des Zivilverfahrens nur über gemeinsam erarbeitete Modellgesetze verwirklichen. Als problematisch erwies sich dabei jedoch, dass die beiden Grundsysteme, die im 18. und 19. Jahrhundert auf deutschem Gebiet erarbeitet worden waren, sich nur schlecht vereinigen ließen. So folgte das preußische System anderen Prinzipien als die wesentlich modernere B.P.O. von 1850. Preußen war jedoch nicht bereit, das zwar modernere aber fremde System einfach zu übernehmen. Daher wurden im 19. Jahrhundert auf deutschem Gebiet diverse Prozessordnungen erlassen, die sich teils aneinander orientierten. Außer den Grundsätzen der Mündlichkeit und Öffentlichkeit, welche als Leitprinzipien in der Reichsverfassung von 1848 festgeschrieben worden waren, mussten die Prozessordnungen keine Gemeinsamkeiten aufweisen.

In Deutschland beeinflussten der vordringende Liberalismus und die Einflüsse der französischen Zivilprozessordnung die Reformen. Die Dispositionsmaxime, Ausdruck der Privatautonomie, wurde gestärkt und eine Konzentration des Verfahrens angestrebt. Ein weiteres Hauptaugenmerk der deutschen Reformer lag auf der Steigerung der Mündlichkeit und der Unmittelbarkeit des Verfahrens. Beide Reformziele fanden ihren Höhepunkt in der B.P.O. von 1850, wonach ausschließlich der mündliche Vortrag Inhalt der Entscheidung werden konnte. Das bedeutete für die Klage, dass der in der Klageschrift enthaltene Sachvortrag im Verfahren erneut gehalten werden musste. Insoweit näherte sich der deutsche Prozess dem englischen (wieder) an. Sowohl im Common Law als auch bei der Equity war seit jeher der mündliche Vortrag entscheidend. Zwar erfolgten die Pleadings schriftlich. Über die Festlegung der Streitpunkte wurde dann aber genauso mündlich verhandelt, wie im Rahmen des Beweisverfahrens vor der Jury.

Die englischen Bestrebungen versuchten den extremen Formalismus, den das Verfahren entwickelt hatte, zu beseitigen. Dazu schaffte man die einzelnen Writs ab. Ein greifbarer Erfolg blieb allerdings aus, weil die gerichte an den alten Bezeichnungen und Voraussetzungen über Umwege weiter festhielten. Erst als sich in der Richterschaft die Auffassung durchsetzte, Formfehler sollten grundsätzlich nicht über Erfolg oder Niederlage im Verfahren entscheiden, entwickelte sich das Common Law vom aktionenrechtlichen Denken in Richtung auf ein stärker materiellrechtlich geprägtes System hin. Im gleichen Maße, in dem man langsam von den strikten Writs abrückte, wurden die Pleading-Regeln liberalisiert. Die Einflüsse auf das Common Law kamen bei diesen Entwicklungen nicht von fremden Rechtsordnungen. Vielmehr entnahm man die Änderungen der Equity, die ohnehin keine Writs kannte und mit Formfehlern seit je her weniger rigoros umgegangen war. Beim Verfahren vor der Chancery wurde vor allem das Writ of subpoena abgeschafft und die Ladung direkt in die Bill of complaint eingetragen. In der Bill selbst war der Kläger zu größerer Genauigkeit und Knappheit verpflichtet.

Die Reformen des 19. Jahrhunderts führten anfänglich weder in Deutschland noch in England zu den erhofften Erfolgen. In Deutschland konnte sich zunächst keine einheitliche Zivilprozessordnung durchsetzen, in England wurde man die Forms 
of action nur formal los. Die Reformbestrebungen zeigten aber die Richtung an und bereiteten in beiden Rechtsordnungen den Umbruch des Verfahrens vor, zu dem es erst in den siebziger Jahren des 19. Jahrhunderts kommen sollte. 


\section{Die Entwicklung der ZPO bis heute}

Da die Verfassung des deutschen Reiches von 1871 das Zivilprozessrecht in die Gesetzgebungskompetenz des Kaiserreichs verlagerte, ${ }^{667}$ musste und konnte eine gemeinsame Zivilprozessordnung geschaffen werden. Dazu wurde im Sommer 1871 der preußische Justizministerial-Entwurf veröffentlicht, der die erste Grundlage zur aktuellen ZPO darstellen sollte.

\section{Der Preußische Justizministerial-Entwurf von 1871}

Der Entwurf von 1871 versuchte eine Vereinheitlichung des Zivilprozessrechts im Deutschen Reich herbeizuführen, was aufgrund der teils sehr verschiedenen Prozessrechte, die in den Ländern galten, eine schwierige Aufgabe darstellte.

Nach dem Entwurf war die Klage schriftlich bei Gericht einzureichen. Sie musste die Bezeichnung der Parteien enthalten sowie die Angabe eines bestimmten Anspruchsgrundes und Streitgegenstandes. Des weiteren war in der Klage ein bestimmter Antrag zu stellen. ${ }^{668}$ Der Kläger konnte auch auf die Feststellung des Bestehens oder Nichtbestehens eines Rechtsverhältnisses klagen. ${ }^{669}$ Die Ladung wurde nicht vom Gericht, sondern vom Kläger vorgenommen, allerdings musste er sich dabei eines Gerichtsvollziehers bedienen. ${ }^{670}$ Erfolgte die Ladung zusammen mit der Klage, war sie in der Klage aufzunehmen. ${ }^{671}$

Auf die Klage hin hatte der Beklagte seine Klageerwiderung in Form eines Schriftsatzes dem Kläger zuzustellen. ${ }^{672}$ Alle weiteren Vorträge der Parteien sollten in der Regel mündlich erfolgen, ${ }^{673}$ wobei der Bezug auf Schriftsätze unzulässig war. ${ }^{674}$ Die Schriftsätze der Parteien hatten demzufolge nur vorbereitenden Charakter. Da nur der mündliche Vortrag Gegenstand der Urteilsfindung war, nahm das Gericht keine Schlüssigkeitsprüfung der Klage vor. ${ }^{675}$ Auch der Protokollierungszwang wurde entschärft, so dass nur noch die Anträge der Parteien und der Gang der Verhandlung schriftlich zu fixieren war. ${ }^{676}$ Besondere Bedeutung erlangte dabei der richterliche Tatbestand, der den Beweis des vor Gericht gesprochenen Wortes lieferte. ${ }^{677}$ Bei der Abfassung des Tatbestandes durfte der Richter auf die Schriftsätze der Parteien zurückgreifen. ${ }^{678}$ Damit wertete man die Schriftsätze, die dem Gedanken nach nur unverbindlich eine vorbereitende Funktion erfüllen sollten, erheblich auf. ${ }^{679}$ Der Prozessbetrieb ging auf die Parteien über. Diese waren auch befugt, einvernehmlich Fristen abzuändern. ${ }^{680}$

\footnotetext{
${ }^{667}$ Art. 4 zu 13

${ }^{668} \S 209$ des Entwurfs von 1871

${ }^{669} \S 210$ des Entwurfs von 1871

${ }^{670} \S 177$ des Entwurfs von 1871

${ }^{671} \S 177$ des Entwurfs von 1871

${ }^{672} \S 222$ des Entwurfs von 1871

${ }^{673} \S 123$ des Entwurfs von 1871

${ }^{674}$ Dahlmanns, S. 80

${ }^{675}$ Bieresborn, S. 345

${ }^{676}$ Nach $§ 139$ des Entwurfs von 1871 waren neben den Anträgen der Parteien Anerkenntnisse, Verzichte und Vergleiche, Zeugen- und Sachverständigenaussagen, Ergebnisse des Augenscheins und Entscheidungen des Gerichts sowie deren Verkündung zu beurkunden.

$677 \S 260$ des Entwurfs von 1871

${ }^{678} § 259$ des Entwurfs von 1871

${ }^{679}$ Bieresborn, S. 339 spricht von den Schriftsätzen als „Grundlage des Prozeßverfahrens“.

${ }^{680} \S \S 177,188$ des Entwurfs von 1871; eine Ausnahme war für Notfristen vorgesehen.
} 


\section{Die RZPO von 1877 und ihre Entwicklung zur aktuellen ZPO}

Nachdem der Entwurf von 1871 allgemein auf positive Resonanz gestoßen war, setzte der Bundesrat eine Kommission zur „definitiven Feststellung des Entwurfs einer Proceßordnung in bürgerlichen Rechtsstreitigkeiten für das deutsche Reich“ ein, die 1872 dem Justizausschuss des Bundesrates eine leicht veränderte Fassung $^{681}$ übergab. Sowohl der Bundesrat als auch anschließend der Reichstag nahmen noch diverse Änderungen vor, bis der Entwurf 1876 nahezu einstimmig das Gesetzgebungsverfahren passierte. ${ }^{682}$ Die RZPO wurde mit Datum vom 30.01.1877 am 19.02.1877 verkündet und trat am 01.10.1879 in Kraft.

\section{a. Klageschrift}

Bezüglich der Klage folgte die RZPO dem Entwurf von 1871. Die entscheidenden Normen haben sich auch bis heute im Wesentlichen nicht geändert, so dass hier die aktuelle Rechtslage besprochen werden kann. ${ }^{683}$ Die Klageerhebung ${ }^{684}$ erfolgt durch Einreichen eines den Erfordernissen des $\S 253 \mathrm{ZPO}^{685}$ entsprechenden Schriftsatzes, § 253 ZPO ( $§ 230$ RZPO). Vor den Amtsgerichten kann dieser auch mündlich zu Protokoll der Geschäftsstelle abgegeben werden, § 495 ZPO. ${ }^{686}$ Die Klage muss die Bezeichnung der Parteien und des Gerichts enthalten, die Angabe des Grundes und des Gegenstandes des erhobenen Anspruchs sowie einen bestimmten Antrag, § 253 II ZPO (§ 230 RZPO). Wird auf einen Streitgegenstand geklagt, der keine Geldsumme darstellt, soll der Wert des Gegenstandes angegeben werden, § 253 II ZPO (§ 230 RZPO).

Die Klage muss den Gegenstand der Klage sowie einen bestimmten Antrag enthalten. Dabei versteht man unter dem Gegenstand der Klage den Inhalt des begehrten Anspruchs, ${ }^{687}$ d.h. der Kläger hat inhaltlich eindeutig festzulegen, welche Entscheidung er begehrt. ${ }^{688}$ Dazu ist erforderlich und genügend, dass er sein Begehren in Form eines bestimmten Antrags fasst. ${ }^{689}$ Der Gegenstand des Anspruchs hat damit seine eigenständige Bedeutung verloren. ${ }^{690}$ Der bestimmte Antrag gibt die Art der Klage und die Angabe des Klagebegehrens wieder, d.h. er umfasst, was der Kläger als Entscheidung ausgesprochen haben will. Dazu muss die begehrte Rechtsfolge so genau und vollständig bezeichnet werden, dass der Antrag als Tenor vollstreckbar wäre. ${ }^{691}$ Zahlungsklagen sind zu beziffern. ${ }^{692}$ Nur in Ausnahmefällen sind unbezifferte Zahlungsanträge zulässig. ${ }^{693}$ Entscheidend ist jedoch, dass in der Klage dem Gericht durch Angabe des anspruchsbegründenden Sachverhalts die Grundlagen für die Bezifferung des Antrages mitgeteilt werden.

\footnotetext{
${ }^{681}$ Coing/Dahlmanns, S. 2679

${ }^{682}$ Bettermann, ZZP 91 (1978), S. 365, 368; Hahn, Materialien Bd. I 1. Teilband, S. 1312

${ }^{683}$ Auf etwaige Unterschiede wird im weiteren Verlauf hingewiesen.

${ }^{684}$ Die ZPO geht vom Verfahren vor dem Landgericht als Regelfall aus.

${ }^{685} \S 253$ ZPO in seiner gegenwärtigen Fassung entspricht im Wesentlichen § 230 RZPO.

${ }^{686}$ Entspricht $\$ 457$ RZPO.

${ }^{687}$ Stein/Jonas/Schumann, § 253 Rn. 48

${ }^{688}$ Thomas/Putzo/Reichold/Hüßtege, $\S 253$ Rn. 8

${ }^{689}$ Stein/Jonas/Schumann, § 253 Rn. 48

${ }^{690} \mathrm{MüKo} /$ Lüke, § 253 Rn. 69; Rosenberg/Schwab/Gottwald, S. 545

${ }^{691}$ Statt aller Thomas/Putzo/Reichold/Hüßtege, § 253 Rn. 11

${ }^{692}$ Thomas/Putzo/Reichold/Hüßtege, § 253 Rn. 11

${ }^{693}$ Dies gilt für den Regelunterhalt (§645 ZPO), Anträge deren Höhe ins Ermessen des Gerichts gestellt werden, insbesondere Schmerzensgeldanträge ( $\$ 847$ BGB) oder wenn dem Kläger die Ermittlung der Höhe seines Anspruchs unzumutbar ist (vgl. BGH NJW 1967, 1420). Siehe auch statt aller Thomas/Putzo/Reichold/Hüßtege, § 253 Rn. 12.
} 
Dies kann die Pflicht zur Angabe einer ungefähren Größenordnung oder einer Mindestsumme beinhalten. ${ }^{694}$

Der Kläger muss bereits in der Klageschrift den Klagegrund vortragen. Damit ist nach inzwischen wohl einhelliger Meinung der Lebenssachverhalt gemeint, aus dem der Kläger die begehrte Rechtsfolge herleiten will. ${ }^{695}$

In der Klage selbst müssen daher nur Tatsachen dargelegt werden, die zur Bestimmung des Streitgegenstandes nach der zweigliedrigen Streitgegenstandslehre geeignet sind. ${ }^{696}$ Demnach ist es möglich, auch wenn es in der Praxis nicht vorkommt, eine Klage nur mit dem Antrag und den Tatsachen, die den Streitgegenstand hinreichend individualisieren, einzureichen. Die ausführliche Klagebegründung muss dann aber spätestens nach Aufforderung durch das Gericht nachfolgen.

Die umfassende Angabe der Tatsachen in der Klageschrift wird jedoch von $\S 130 \mathrm{Nr}$. 3 ZPO als Ordnungsvorschrift gefordert und kann von Vorteil sein, um ggf. Verspätungsfolgen zu entgehen. ${ }^{697} \S 138$ I ZPO verpflichtet die Parteien darüber hinaus, ihre Angaben vollständig und wahrheitsgemäß zu machen. Schließlich kann ein Versäumnisurteil zugunsten des Klägers nur ergehen, wenn seine Klage schlüssig, das heißt in diesem Zusammenhang auch vollständig vorgetragen wurde, § 331 II ZPO.

Die Schlüssigkeit der Klage ist keine Frage der Zulässigkeit, so dass das Gericht bei Eingang der Klage nur prüft, ob der Kläger überhaupt Tatsachen zum Klagegrund angegeben hat. Dies ergibt sich im Umkehrschluss aus $\S \S 139$ I, 264 Nr. 1 ZPO, die eine Ergänzung des Tatsachenvortrages zulassen. Fehlt der Tatsachenvortag gänzlich, ist die Klage formell fehlerhaft und aufgrund einer fehlenden Prozessvoraussetzung durch Prozessurteil abzuweisen ${ }^{698}$ Rechtsausführungen sind in der Klageschrift, wie im gesamten Parteivortrag nicht erforderlich. Es gilt der Grundsatz da mihi factum, dabo tibi ius. ${ }^{699}$ Das Anführen von Rechtsansichten ist jedoch nicht verboten. Vielmehr kann es in bestimmten Fällen sogar angebracht sein, um das Gericht bei der Rechtsfindung zu unterstützen und vor Fehlern zu bewahren. ${ }^{700}$ Eine vollständige Nichtberücksichtigung durch das Gericht kann unter Umständen eine Verletzung des rechtlichen Gehörs gem. Art. 103 I GG darstellen. ${ }^{701}$

Weitere formelle Anforderungen an die Klageschrift ergeben sich aus der Verweisung in $\S 253$ IV ZPO auf $\S 130$ ZPO. Soweit nicht $\S 253$ II, III und V ZPO als Spezialvorschriften vorgehen, bestimmt $\S 130$ ZPO auch den Inhalt der Klageschrift. Dabei handelt es sich dem Wortlaut nach um eine Sollvorschrift, die jedoch von der Rechtsprechung teilweise zur zwingenden Norm verdichtet wurde. Dies gilt jedenfalls für Nr. $1^{702}$ und $6^{703}$, da sich nur so der Zweck der Verfahrensförderung erreichen lässt. Nach $\S 130 \mathrm{Nr}$. 1 ZPO müssen die Parteien und ihre gesetzlichen Vertreter nach Namen, Stand oder Gewerbe, Wohnort und Parteistel-

\footnotetext{
${ }^{694}$ BGH NJW, 1992, 311

${ }^{695}$ BGHZ 22, 254 (255); Michel/von der Seipen, S. 119; MüKo/Lüke, § 253 Rn. 74; Rosenberg/Schwab/Gottwald, S. 545; Stein/Jonas/Schumann, § 253 Rn 124; Thomas/Putzo/Reichold/Hüßtege, § 253 Rn. 10

${ }^{696}$ Stein/Jonas/Schumann, § 253 Rn 128

${ }^{697}$ Bieresborn, S. 349, Rn. 20

${ }^{698}$ BGH NJW-RR 1989, 396

${ }^{699}$ Bergerfurth, Rn. 120; Michel/von der Seipen, S. 122

${ }^{700}$ BGH NJW 1974, 1866

${ }^{701}$ BVerfG NJW-RR 1993, 383

${ }^{702}$ BGHZ 102, 335; BVerwG NJW 1999, 2609

${ }^{703}$ BAG NJW 1990, 3165
} 
lung bezeichnet werden, das Gericht und der Streitgegenstand angeführt und die Zahl der Anlagen angegeben werden. Bezüglich der Nämlichkeit der Parteien kommt es allerdings nur darauf an, dass eine Bezeichnung erfolgt, „die Zweifel hinsichtlich der Identität und der Stellung der Partei ausschließt und gewährleistet, dass sich die Partei für jeden Dritten ermitteln lässt. ${ }^{\text {“704 }}$

Gem. § 130 Nr. 6 ZPO ist die Klage eigenhändig zu unterschreiben. ${ }^{705}$ Es soll sichergestellt werden, dass jemand Verantwortung für den Schriftsatz übernimmt. ${ }^{706}$ Beim Anwaltsprozess muss dies der Anwalt tun. ${ }^{707}$ Eine nicht unterschriebene Klage wird als bloßer Entwurf gewertet und nicht zugestellt. ${ }^{708}$ Schließlich soll der Kläger in der Klage die Beweismittel bezeichnen, die er verwenden möchte, $\S 130$ Nr. 5 ZPO. Formale Mängel in der Klage sind grundsätzlich heilbar. Auf Mängel beim notwendigen Inhalt muss das Gericht zweckmäßigerweise schon vor der Zustellung an den Beklagten hinweisen, damit der Mangel geheilt werden kann. Alle Mängel sind aber bis zum Abschluss der mündlichen Verhandlung heilbar. ${ }^{709}$ Soweit mit der Klage eine Frist gewahrt wird, greift eine Heilung nur ex nunc ein. ${ }^{710}$ Findet keine Heilung statt, wird die Klage als unzulässig abgewiesen. ${ }^{711}$ Mängel beim nicht notwendigen Inhalt betreffen die Zulässigkeit der Klage nicht, können aber zu Verzögerungen im Verfahren führen, die zu Lasten des Klägers gehen und möglicherweise eine Fristwahrung nach $\S 167$ ZPO in Frage stellen. ${ }^{712}$

Sobald die Klage bei Gericht eingereicht ist, ist das Verfahren anhängig. ${ }^{713}$ Das Einreichen geschieht durch Zusenden oder Abgabe eines Schriftsatzes bei Gericht. Grundsätzlich ist seit der Einführung des $\S 130$ a ZPO auch das Einreichen in elektronischer Form möglich, soweit dazu Rechtsverordnungen schon bestehen. Derzeit regelt lediglich die ERVVOBGH den elektronischen Rechtsverkehr beim BGH. Danach können Schriftsätze in bestimmten vorgeschriebenen Formaten als Anhang einer E-Mail an das Gericht gesandt werden. Die Funktion der eigenhändigen Unterschrift übernimmt die elektronische Signatur, die sich nach dem SigG bestimmt.

Erfolgt die Zustellung der Klage „demnächst“, also ohne besondere Verzögerung $^{714}$, werden Fristen mit Einreichung der mangelfreien Klageschrift gewahrt, $\S \S 207,167$ n.F. ZPO. Dies gilt gleichermaßen für Ausschlussfristen, wie für die Hemmung der Verjährung, § 204 BGB.

$\mathrm{Ab}$ Rechtshängigkeit der Klage kann dieselbe Streitsache zwischen den Parteien nicht vor einem anderen Gericht geltend gemacht werden, § 261 III Nr. 1 ZPO. Dazu muss zum einen die Nämlichkeit der Parteien und zum anderen der Streitgegenstand feststehen. Der Streitgegenstand der Leistungsklage umfasst den der

\footnotetext{
${ }^{704}$ BGH MDR 1989, 428; BHG NJW 19771686

${ }^{705}$ Dies lässt sich auch aus $\S \S 253$ V ZPO i.V.m. $§ 126$ I BGB herleiten. Ausführlich dazu KunzSchmidt, NJW 1987, $1296 \mathrm{ff}$

${ }^{706}$ BGH VersR 1980, 331

${ }^{707}$ RGZ 151, 82, 85 und seither ständig.

${ }^{708}$ RGZ 151, 82, 84; BGH NJW 1985, 328, 329; Kunz-Schmidt, NJW 1987, 1296, 1297

${ }^{709}$ Zöller/Greger, § 253 Rn. 23

${ }^{710}$ BGHZ 22, 257

${ }^{711}$ Bei fehlendem bestimmtem Antrag oder der Bezeichnung von Gegenstand und Grund sogar, wenn sich der Beklagte rügelos eingelassen hat; BGH NJW 1972, 1373; vgl. auch Zöller/Greger, $\S 253 \mathrm{Rn} .23$.

${ }_{712}$ Zöller/Greger, § 253 Rn. 24

${ }^{713}$ Baumbach/Lauterbach/Albers/Hartmann, § 253 Rn. 105; § 261 Rn. 1

${ }^{714}$ Baumbach/Lauterbach/Albers/Hartmann, § 207 Rn. 3
} 
positiven und negativen Feststellungsklage, nicht jedoch umgekehrt. ${ }^{715}$ Die entgegenstehende Rechtskraft ist von Amts wegen zu beachten und führt zur Abweisung der Klage als unzulässig. ${ }^{716} \S 167$ n.F. ZPO findet auf die entgegenstehende Rechtshängigkeit keine Anwendung. ${ }^{717}$

\section{b. Zustellung}

Mit Zustellung der Klageschrift gilt die Klage als erhoben, und Rechtshängigkeit tritt ein, $\S \S 261,262$ ZPO.

Nach der RZPO von 1877 fand die Zustellung nicht von Amts wegen statt, sondern oblag dem Kläger, der sich dazu in der Regel ${ }^{718}$ eines Gerichtsvollziehers bediente, $\S 152$ RZPO. Veranlasste der Kläger die Terminsbestimmung bei Gericht, erfolgte auch die Ladung des Beklagten durch den Kläger. ${ }^{719}$ Bestimmte das Gericht den Termin, nahm es zum Teil auch die Ladung vor. ${ }^{720}$

Bei der ersten großen Änderung der RZPO wurden 1898 die Vorschriften über die Zustellung so gelockert, dass ein Gerichtsvollzieher nicht mehr zwangsläufig einzuschalten war, § 166 RZPO 1898. Im dritten Reich führte man 1943 den Amtsbetrieb ein, wonach die Terminierung, Ladung und Zustellung von Amts wegen erfolgte. ${ }^{721}$ Nach dem zweiten Weltkrieg wurde die ZPO allerdings weitestgehend wieder so hergestellt, wie sie vor 1933 existiert hatte, so dass es zur endgültigen Einführung der Terminsbestimmung, Ladung und Zustellung von Amts wegen erst $1976 \mathrm{kam}, \S \S 214,216,271$ I und 274 I ZPO.

Dies ist auch die noch heute gültige Rechtslage. Zustellung ist dabei der in gesetzlicher Form zu bewirkende und $\mathrm{zu}$ beurkundende Akt, der dem Adressaten die Möglichkeit gibt, von einem Schriftstück Kenntnis zu nehmen. ${ }^{722}$

Ist die Klageschrift bei Gericht eingegangen, wird sie von diesem unverzüglich zugestellt, $§ \S 271$ I, 253 I, 270 I, 209 ff., 208, 266 ff. ZPO. Dies geschieht allerdings erst, wenn der Kläger den Gerichtskostenvorschuss eingezahlt hat, $\S 65$ I 1 GKG. Insofern hat sich ein Restbestand des Parteibetriebs gehalten.

Mit der Zustellung der Klageschrift wird der Beklagte aufgefordert, sich im landgerichtlichen Verfahren einen Rechtsanwalt zu bestellen, wenn er sich gegen die Klage verteidigen will, § 271 II ZPO. Bestimmt das Gericht die Durchführung eines schriftlichen Vorverfahrens, ist dem Beklagten mitzuteilen, dass er seine Verteidigungsabsicht binnen zwei Wochen nach Zustellung bei Gericht anzeigen muss und binnen einer weiteren vom Gericht festzusetzenden Frist von mindestens zwei Wochen schriftlich auf die Klage zu erwidern, § 276 I ZPO. Gleichzeitig ist er über die Folgen der Säumnis zu belehren, § 276 II ZPO. Entscheidet sich das Gericht für einen frühen ersten Termin, § 275 ZPO, kann es den Beklagten auffordern, seine Verteidigung binnen einer Frist von wenigstens zwei Wochen bei Gericht einzureichen, $\S \S 275$ I 1, 277 III ZPO. Diese Frist kann auch im Termin gesetzt werden, $\S \S 275$ III, 277 III ZPO. Die Verfügung des Gerichts wird mit der Klageschrift zugestellt.

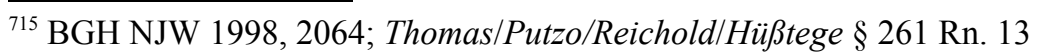

${ }^{716}$ Thomas/Putzo/Reichold/Hüßtege § 261 Rn. 13

${ }^{717}$ BayObLG MDR 1990, 632 zu $§ 270$ III a.F. ZPO

${ }^{718}$ Siehe zur Postzustellung § 161 RZPO.

${ }^{719}$ Damrau, S. 13

${ }^{720}$ Z.B. § 195 RZPO; Umfassend zu den einzelnen Fällen Damrau, S. 13 ff..

${ }^{721} \S 2$ I, III der Vereinfachungsnovelle von 1943 (1. Abschnitt)

${ }^{722}$ Rosenberg/Schwab/Gottwald, S. 403
} 
Grundsätzlich erfolgt die Zustellung im Amtsbetrieb, § 166 II ZPO und wird durch den Gerichtswachtmeister oder Postbediensteten ausgeführt, § 176 I ZPO. Die Zustellung ist zu beurkunden, $\S \S 182$ ZPO.

Die Zustellung erfolgt regelmäßig durch Übergabe der Klageschrift an die Partei, ihren gesetzlichen Vertreter, die zur Vertretung berufenen Organe juristischer Personen oder den (Prozess-) Bevollmächtigten, $\S \S 170$ ff. ZPO.

Ist die Zustellung per Übergabe nicht möglich, eröffnet die ZPO Möglichkeiten der Ersatzzustellung, $\S 178$ ( $\S 208,181$ a.F.) ZPO, bei Erfolglosigkeit durch Niederlegung beim örtlichen Amtsgericht oder Postanstalt bei gleichzeitiger Benachrichtigung des Adressaten, $\S 181$ ( $\S$ 208, 182 a.F.) ZPO. Die Zustellung im Ausland ist nur erforderlich, wenn die Zustellung im Inland unmöglich ist, also nicht, wenn sich der Zustellungsadressat (kurzfristig) im Inland aufhält oder im Inland eine Zweitwohnung oder ein Geschäftslokal unterhält. Muss im Ausland zugestellt werden, folgt das Verfahren der EuZustVO, den jeweiligen Staatsverträgen und den Verwaltungsvorschriften der Rechtshilfeordnung in Zivilsachen (ZRHO). ${ }^{723}$ Nur wenn der Aufenthaltsort einer Partei unbekannt oder die Zustellung im Ausland unausführbar oder nicht erfolgversprechend ist, kann eine öffentliche Zustellung erfolgen, $\S 185$ ZPO. Die öffentliche Zustellung ist keine tatsächliche Zustellung, sondern Zustellungsfiktion. ${ }^{724}$

Eine grundlegende Änderung der Zustellungsvorschriften ergibt sich durch das Zustellungsreformgesetz vom 25.06.2001, das zum 01.07.2002 in Kraft getreten ist. Das Zustellungsrecht wurde neu gefasst. Die in praxi ohnehin weit überwiegende Zustellung von Amts wegen nimmt den Hauptteil der Neuregelung ein. ${ }^{725}$ Nunmehr enthält das Gesetz in $\S 166$ I ZPO eine Legaldefinition der Zustellung. ${ }^{726}$ Die Rückwirkung der Zustellungsfolgen, wenn die Zustellung demnächst erfolgt ist, findet sich in $\S 167$ ZPO. Die Zustellungsformen blieben weitestgehend gleich. §175 ZPO erlaubt ausdrücklich die Zustellung per Einschreiben mit Rückschein. Die Ersatzzustellung wurde lediglich modernisiert und leicht vereinfacht. ${ }^{727}$ Die Voraussetzungen der öffentlichen Zustellungen wurden zwar nicht entscheidend verändert, die Durchführung hingegen wurde wesentlich erleichtert. Insbesondere ist die Veröffentlichung im Bundesanzeiger nur noch auf gerichtliche Anordnung hin erforderlich, § 187 ZPO.

Geändert wurde auch die Regelung zur Benennung eines Zustellungsbevollmächtigten im Inland, wenn eine Partei ihren Wohnsitz im Ausland hat. Wo $\S 174$ II ZPO a.F. die Pflicht zur Benennung normierte, verlangt $\S 184$ I 1 ZPO n.F. eine Benennung nur noch, wenn die Zustellung im Rechtshilfeverfahren oder im dezentralen direkten Behördenverkehr vorgenommen wird. Die Entscheidung steht im Ermessen des Gerichts.

In Folge der Entwicklung neuer Telekommunikationsmedien normiert $§ 174$ ZPO die Zustellung gegen Empfangsbekenntnis mit modernen Kommunikationsmitteln. So kann an einen Anwalt oder eine Person, bei der aufgrund ihres Berufes von einer erhöhten Zuverlässigkeit ausgegangen werden kann, per Fax oder als elektronisches Dokument (derzeit E-Mail mit Anhang) zugestellt werden. Die Zustellung eines elektronischen Dokuments kann auch an andere Verfahrensbeteiligte erfolgen, wenn sie dazu ihr Einverständnis erklärt haben, § 174 III 2 ZPO.

\footnotetext{
${ }^{723}$ Ausführlich dazu Geimer, Rn. 2132

${ }^{724}$ Maunz/Dürig, Art. 103 I Rn. 47

${ }^{725}$ Hartmann, NJW 2001, 2577, 2581

${ }^{726}$ Zustellung ist die Bekanntgabe eines Schriftstücks an eine Person in bestimmter Form.

${ }^{727}$ Vgl. $§ 178$ ff. ZPO
} 
Die Zustellung ins Ausland folgt für das EU-Ausland weiterhin der Zustellungsverordnung, $\S 183$ III ZPO ${ }^{728}$ und dem $\S \S 1067$ ff. ZPO. Im sonstigen Ausland kann, soweit kein Staatsvertrag entgegensteht, per Einschreiben mit Rückschein oder Rechtshilfeersuchen zugestellt werden, § 183 I Nr. 1-3, II ZPO.

\section{c. Verfahrenseinleitung im Mahnverfahren ${ }^{729}$}

Der Gläubiger einer Geldleistung kann auch, statt zu klagen, einen Mahnbescheid beantragen, § 688 ZPO. Dazu muss er am Amtsgericht an seinem eigenen allgemeinen Gerichtsstand ${ }^{730}$ einen auf Erlass eines Mahnbescheids gerichteten Antrag gem. § 690 ZPO stellen. Der Antrag muss die Bezeichnung der Parteien und ggf. ihrer gesetzlichen Vertreter und Prozessbevollmächtigten, die Bezeichnung des Gerichts, des Anspruchs unter bestimmter Angabe der verlangten Leistung und die Erklärung darüber, dass der Anspruch nicht von einer Gegenleistung abhängig ist, enthalten sowie das für das streitige Verfahren sachlich zuständige Gericht bezeichnen, §690 I ZPO. Schließlich muss der Antrag eigenhändig unterzeichnet werden, § 690 II ZPO. Soweit bei dem jeweiligen Amtsgericht für den Antrag Vordrucke vorhanden sind, müssen diese ausgefüllt werden, §§ 702, 703 c ZPO. Das Amtsgericht ${ }^{731}$ erlässt den Mahnbescheid nach formeller, nicht jedoch materieller Prüfung. ${ }^{732}$ Der Mahnbescheid enthält unter anderem ${ }^{733}$ die Aufforderung an den Antragsgegner, binnen zwei Wochen den Antragsteller zu befriedigen oder Widerspruch einzulegen, $\S 692$ I Nr. 3 ZPO. Der Mahnbescheid wird von Amts wegen zugestellt. Zur Fristwahrung gilt $§ 167$ ZPO.

Wird hingegen gegen den Mahnbescheid Widerspruch bzw. gegen den Vollstreckungsbescheid Einspruch eingelegt, geht die Sache in ein streitiges Verfahren über. Das Verfahren wird an das im Mahnbescheid angegebene Gericht oder ein anderes von den Parteien übereinstimmend bezeichnetes Gericht abgegeben, $\S \S 696$ III, 700 I ZPO. Das Gericht fordert daraufhin den Antragsteller auf, seinen Anspruch binnen zwei Wochen in einer Klagebegründung, die der Form einer Klageschrift entspricht, zu begründen, §§ 697 I und 700 III i.V.m. § 697 I ZPO. $\mathrm{Ab}$ diesem Zeitpunkt folgt der Prozess dem normalen Gang eines streitigen Verfahrens.

\section{d. Der Urkundenprozess ${ }^{734}$}

Klagt der Kläger auf Zahlung einer Geldsumme und kann er alle anspruchsbegründenden Tatsachen durch Urkunden beweisen, hat er die Möglichkeit mit seiner Klage ein Urkundenverfahren einzuleiten, gem. $\S \S 592$ ff. ZPO.

In der Klage muss der Kläger ausdrücklich oder konkludent ${ }^{735}$ kenntlich machen, dass er ein Urkundenverfahren anstrengen will, § 593 I ZPO. Diese Erklärung kann er vor Zustellung der Klage an den Beklagten noch nachholen. ${ }^{736}$

\footnotetext{
${ }^{728}$ Verordnung des Rates Nr. 1348/2000

${ }^{729}$ Eine umfassende Darstellung findet sich bei Knöringer, S. $278 \mathrm{ff}$.

${ }^{730} \S 689$ II ZPO begründet insofern eine ausschließliche Zuständigkeit.

${ }^{731}$ Der Bescheid wird durch den Rechtspfleger erlassen, § 20 Nr. 1 RPflG.

${ }^{732}$ Vgl. § 692 I Nr. 2 ZPO.

${ }^{733}$ Siehe dazu $\S 692$ I ZPO

${ }^{734}$ Weitere Besonderheiten gelten für den Wechsel- und Scheckprozess als Unterarten des

Urkundenprozesses. Siehe umfassend zum Urkundenverfahren Oberheim, S. 296 ff.

${ }^{735}$ Anders/Gehle, Rn. 628, Oberheim, § 13 Rn. 6

${ }^{736}$ Anders/Gehle, Rn. 641
} 
Der Kläger muss der Klageschrift oder einem späteren Schriftsatz Urkunden zum Beweis seiner Behauptungen beifügen, $\S 593$ II ZPO. ${ }^{737}$

\section{e. Weitere erhebliche Neuerungen und Änderungen}

Obwohl in der RZPO das Mündlichkeitsprinzip galt, § 119 RZPO, wurden schon 1877 einige schriftliche Elemente fixiert. Neben der Klage musste auch die Klageerwiderung schriftlich erfolgen, § 244 RZPO, sowie unter bestimmten Umständen vorbereitende Schriftsätze ausgetauscht werden, § 120 RZPO (heute § 129 ZPO). Eine Bezugnahme auf Schriftsätze in der mündlichen Verhandlung war jedoch grundsätzlich unzulässig, $\S 128$ RZPO.

1898 erfolgte eine Anpassung der RZPO an das neue BGB mit vereinzelten Änderungen. ${ }^{738}$ Obwohl es viele Befürworter gab, verzichtete man auf die Einführung eines Vortermins zur Erledigung des Streits ohne streitige Verhandlung. ${ }^{739}$

Zwei wesentliche Änderungen der RZPO nahm man 1909 vor. Zum einen wurde das richterliche Fragerecht neu geregelt und gestärkt ${ }^{740}$, zum anderen eine Bezugnahme auf Schriftsätze zugelassen, soweit keine Partei widersprach und das Gericht sie für angemessen hielt. ${ }^{741}$

Die „Emminger“-Novelle von 1924 stärkte das richterliche Fragerecht ${ }^{742}$ und schwächte im Gegenzug den Verhandlungsgrundsatz. ${ }^{743}$ Obwohl § 139 ZPO lediglich den Gesetzeszweck hatte, unklare Sachverhalte zu klären und damit das Verfahren zu beschleunigen, weiteten die Gerichte die Vorschrift in praxi zu einer umfangreichen Verpflichtung zur Sachverhaltsaufklärung aus. ${ }^{744}$ Inzwischen versteht man $\S 139$ ZPO als Ausprägung des sozialen Rechtsstaates, wonach das Gericht Fürsorgepflichten einzuhalten und die Waffengleichheit der Parteien zu garantieren hat. ${ }^{745}$

Die sog. Vereinfachungsnovelle von 1976 nahm zum Teil erhebliche Änderungen vor. Sie fasste die allgemeinen Prozessförderungspflichten der Parteien neu, ${ }^{746}$ führte die Unterscheidung zwischen schriftlichem Vorverfahren und frühem ersten Termin ${ }^{747}$ sowie einen Sühneversuch als Sollvorschrift ein und weitete die Präklusionsvorschriften aus. ${ }^{748}$

Weitere Änderungen erfuhr die ZPO durch das Zivilprozessreformgesetz 2001. ${ }^{749}$ Diese umfassende Reform berührte die Einleitung des Verfahrens nur peripher. Neben der Einführung einer obligatorischen Güteverhandlung vor der mündlichen Verhandlung, § 279 ZPO, entscheidet beim Landgericht nun regelmäßig ein origi-

\footnotetext{
${ }^{737}$ Dabei sind Indizurkunden (BGH NJW 1985, 2953: Urkunden, aus denen auf das Bestehen des Anspruchs rückgeschlossen werden kann.) ausreichend für die Statthaftigkeit der Verfahrensart. Auch wenn alle Tatsachen zwischen den Parteien unstreitig sind, muss der Kläger zumindest eine Urkunde vorlegen, sonst ist die Klage als im Urkundenverfahren unstatthaft abzuweisen.

Vgl. BGH NJW 1974, 1199; BGHZ 62, 286; Anders/Gehle, Rn. 631; Schellhammer, Rn. 2953; aA. OLG Jena MDR 1997, 975

${ }^{738}$ So z.B. die vereinfachte Zustellung von Amts wegen.

${ }^{739}$ Dannreuther, S. 168

${ }^{740} \S 502$ I ZPO von 1909

${ }^{741} \S 502$ II ZPO von 1909 (heute $§ 137$ III ZPO)

${ }^{742} \S 139 \mathrm{ZPO}$

${ }^{743}$ Dannreuther, S. 354 m. w. N

${ }^{744}$ Bettermann, ZZP 91 (1978), S. 365, 389 f.

${ }^{745}$ Umfassend dazu Stürner, Aufklärungspflicht, S. 31 ff.zur verfassungsrechtlichen Grundlage,

S. 62 ff. zu $§ 139$ ZPO.

${ }^{746} \S 282$ I-III ZPO

$747 \S \S 272$ II, 275, 276

748 \&279 ZPO von 1976

${ }^{749}$ Ausführlich dazu Hartmann, NJW 2001, 2578 ff.
} 
närer Einzelrichter, § 348 ZPO. Die richterlichen Prozessleitungspflichten nach $\S 139$ ZPO wurden noch einmal ausgedehnt. Alle weiteren Änderungen der hier behandelten Materie sind redaktioneller Art.

\section{Rechtsvergleichende Betrachtung}

Seit dem Ende des 19. Jahrhunderts entwickelten sich in Deutschland und England Prozessrechtssysteme, die nur aus ihrer Tradition heraus vollumfänglich verständlich sind. Beide Systeme müssen aber dem praktischen Rechtsalltag gerecht werden. In diesem Spannungsfeld von Tradition und Reform haben sich bis zur Gegenwart zwei hochentwickelte Systeme herausgebildet.

Im Folgenden wird das deutsche und englische Zivilverfahren der Gegenwart vergleichend untersucht, wobei auf Unterschiede zwischen den RSC/CCR und CPR 1998 gesondert hingewiesen wird.

\section{a. Inhaltliche Anforderungen an das einleitende Schriftstück}

Die Anforderungen an das Schriftstück lassen sich in subjektive, objektive und formale Aspekte unterteilen.

\section{aa. In subjektiver Hinsicht}

In formeller Hinsicht muss jedes einleitende Schriftstück die Parteien bezeichnen. Dies ist von Bedeutung, da die Parteien aktiv- und passivlegitimiert sein müssen. Ferner bestimmt sich nach den Parteien die Rechtskraft in subjektiver Hinsicht.

Die Bezeichnung erfolgt in beiden Systemen grundsätzlich durch Nennung des Namens und der Anschrift. Weitere Zusätze sind in England nur erforderlich, wenn Verwechslungsgefahr besteht, oder wenn die Prozessfähigkeit wegen Minderjährigkeit bzw. Unzurechnungsfähigkeit betroffen wäre. ${ }^{750}$ Mehrere Beklagte sind durchzunummerieren.

Das deutsche Recht verlangt, dass die Parteien nach Namen, Stand oder Gewerbe bezeichnet werden, $\S 130 \mathrm{Nr} .1$ ZPO. Auch für $\S \S 253$ II, $130 \mathrm{Nr} .1$ ZPO gilt aber, dass präzisere Angaben als Name und Anschrift nur erforderlich sind, wenn es andernfalls zu Verwechslungen kommen kann. ${ }^{751}$ Denn kommt es in Folge von mangelhafter Personalisierung des Gegners zu Verzögerungen, so geht dies zu Lasten des Klägers. ${ }^{752}$ Gleiches gilt, wenn der gesetzliche Vertreter einer nicht prozessfähigen Person nicht aufgeführt wird. ${ }^{753}$ Ist es unzumutbar, den Namen oder die Anschrift des Beklagten herauszufinden, kann die Angabe in beiden Systemen unterbleiben. ${ }^{754}$

Handelt eine der Parteien als Partei kraft Amtes, so ist dies im deutschen Recht im Rubrum zu vermerken. ${ }^{755}$ Besteht eine Prozessstandschaft oder klagt der Kläger aus abgetretenem Recht, muss dies in der Begründung aufgeführt werden. Gleichermaßen verlangen rr. 16.2 (3) und (4) CPR 1998, dass in der Claim form angegeben wird, ob eine Partei in ihrer Funktion als representative capacity tätig wird und um welche Funktion es sich handelt.

\footnotetext{
${ }^{750}$ S.o. B.II.2.a.aa. und B.III.2.a.aa; C.IV.2.a.

751 „Notwendig und ausreichend ist eine Bezeichnung, die Zweifel hinsichtlich der Identität und der Stellung der Partei ausschließt und gewährleistet, dass sich die Partei für jeden Dritten ermitteln lässt.“" BGH MDR 1989, 428; BGH NJW 19771686

${ }^{752}$ Baumbach/Lauterbach/Albers/Hartmann, § 130 Rn. 29

${ }^{753}$ Baumbach/Lauterbach/Albers/Hartmann, § $130 \mathrm{Rn} .8$

754 para 2.3 PD 16; LG Kassel NJW-RR 1991, 382

${ }^{755}$ Stein/Jonas/Schumann, § 253 Rn. 35
} 
Bezüglich der Bezeichnung der Parteien sind demnach die Regelungen in Deutschland und England weitgehend inhaltsgleich. Die Genauigkeit, mit der die Parteien bezeichnet werden müssen, hängt von der Notwendigkeit ab, Verwechslungen zu vermeiden und schutzbedürftige Personen zu schützen.

Hat der Kläger einen Prozessbevollmächtigten, ist dieser sowohl in England als auch in Deutschland mit Namen und Anschrift zweckmäßigerweise aufzuführen, insbesondere, wenn weitere Korrespondenz mit ihm erfolgen soll.

Weiterhin muss in beiden Systemen das angerufene Gericht aufgeführt werden. In England soll am High Court auch die Abteilung eingetragen werden. Wird ein deutsches Verfahren im Mahnverfahren eingeleitet, ist im Mahnantrag schon das Gericht anzuführen, an das die Sache im Falle des streitigen Verfahrens abgegeben werden soll.

Die Nennung des Gerichts ergibt sich aus der in beiden Systemen gültigen Dispositionsmaxime: Es obliegt dem Kläger, ob und wo er seinen Anspruch geltend macht. Ruft er ein unzuständiges Gericht an, hat er auch die Folgen zu tragen. Dies sind sowohl in Deutschland als auch in England vor allem Kostenfolgen. ${ }^{756}$ Außerdem wird im englischen Recht die Verjährung nicht mit Ausstellung der Claim form gehemmt, da das unzuständige Gericht die Klage abweisen kann, wenn die Claim form nur zur Fristwahrung, schuldhaft und nicht bona fide beantragt wurde. ${ }^{757}$

\section{bb. In objektiver Hinsicht}

In objektiver Hinsicht muss eine Klage dem Gegner und dem Gericht deutlich machen, was der Kläger vom Beklagten will und auf welchen tatsächlichen Sachverhalt er dies stützt.

Beide Systeme haben zunächst gemeinsam, dass die Parteien den Rechtsgrund der Klage bzw. Verteidigung nicht anführen müssen. Es gilt der Grundsatz da mihi factum, dabo tibi ius. Den Parteien obliegt nur der Tatsachenvortrag.

\section{(1) Begehrter Rechtsausspruch}

$\S 253$ II Nr. 2 ZPO verlangt vom Kläger die Angabe eines bestimmten Antrags. Das heißt, der Kläger muss grundsätzlich genau angeben, welche Entscheidung er vom Gericht begehrt. Das Gericht ist an den Antrag gebunden. Es kann zwar weniger, aber nie mehr als begehrt, zusprechen. Dem Kläger steht frei, seinen Antrag bis zum Schluss der mündlichen Verhandlung zu ändern. Auf fehlerhafte Anträge muss das Gericht nach $\S 139$ ZPO hinweisen. Unbezifferte Zahlungsanträge sind nur ausnahmsweise und oft unter Angabe einer Größenordnung oder Mindestsumme zu nennen.

Auch im englischen Verfahren muss der Kläger sein Begehr genau bezeichnen. Im Verfahren nach den RSC tat er dies im General indorsement auf der Rückseite des Writs. Nach den CPR 1998 ist der begehrte Rechtsbehelf (Remedy) nach den Brief Details of Claim einzutragen. Im Gegensatz zu früher ist das Gericht nach den CPR 1998 an den in der Claim form angeführten Rechtsbehelf nicht mehr gebunden. Es kann auch andere Rechtsbehelfe zusprechen. Diese Befugnis stellt einen bemerkenswerten Eingriff in die Dispositionsmaxime dar und ist im deutschen Recht durch $\S 308$ I ZPO ausgeschlossen. Bei funktionaler Vergleichung ist die Neuerung allerdings nicht mehr ganz so erstaunlich. Es soll dafür gesorgt

\footnotetext{
${ }^{756}$ rr. 30.2 (2) CPR 1998; ss. 40 (1), 42 (1) County Court Act 1984; ss. 51 (8), (9) Supreme Court Act 1981; § 281 III ZPO

${ }^{757}$ Vgl. dazu Restick v. Crickmore [1994] 1 WLR 420 (C.A.).
} 
werden, dass gerechte Urteile zustande kommen, sei es, indem das Gericht darauf hinwirkt, dass die Parteien die richtigen Anträge stellen oder durch die Lockerung der Dispositionsmaxime dahingehend, dass das Gericht nicht mehr absolut an den Antrag gebunden ist. Dabei ist die Leitidee, Parteien $\mathrm{zu}$ helfen, die anwaltlich nicht oder ggf. schlecht ${ }^{758}$ vertreten sind. Funktional entspricht die Befugnis des englischen Richters, über den Antrag hinaus zu gehen, der Hinweispflicht im deutschen Verfahren. ${ }^{759}$

Wird in England auf eine Geldschuld geklagt, muss die Claim form die Summe enthalten (Statement of value). Die Gesamtsumme des Anspruchs inklusive Zinsen muss auch eingetragen werden (Amount claimed). Dadurch wird wie im deutschen Verfahren Klarheit bezüglich der Höhe der Klageforderung geschaffen und der Streitwert festgelegt. In England ist der Streitwert allerdings nicht primär für die sachliche Zuständigkeit, sondern für die Einteilung in die einzelnen Tracks erheblich. Unbezifferte Klageanträge sind auch im Verfahren nach den CPR 1998 möglich, allerdings muss der Kläger eine ungefähre Größenordnung angeben, damit das Verfahren in den entsprechenden Track eingeordnet werden kann. Wie in Deutschland ist das Gericht an die angegebene Größenordnung nicht gebunden. Es kann auch mehr zusprechen.

\section{(2) Tatsachenvortrag}

Im deutschen Zivilverfahren muss der Kläger klagebegründende Tatsachen vortragen. Für die Zulässigkeit der Klage ist es ausreichend, wenn Tatsachen angegeben sind, die eine Bestimmung des Streitgegenstandes ermöglichen (§ 253 II Nr. 2 ZPO: Klagegrund). Für die Schlüssigkeit müssen alle anspruchsbegründenden Tatsachen spätestens am Ende der mündlichen Verhandlung substantiiert dargelegt werden, sonst kommt es zum klageabweisenden Sachurteil. Der Kläger soll jedoch schon in der Klageschrift umfassend vortragen, $\S 130 \mathrm{Nr}$. 3 ZPO und ist zur Vollständigkeit verpflichtet, § 138 ZPO. Tut er dies nicht, droht die Präklusion.

Der Kläger im englischen Prozess muss auf der Claim form (Writ) nur den Anspruch grob umreißen, um dem Beklagten anzuzeigen, aus welchem Lebenssachverhalt und welchem rechtlichen Bereich er verklagt wird, sogenannte Brief Details of Claim (General indorsement of claim). Weitergehende Ausführungen sind nicht erforderlich. Allerdings steht es dem Kläger frei, auch schon genaue Ausführungen in der Claim form vorzunehmen und seine Klage zu begründen. In der Regel ist dafür allerdings schon aus Platzgründen ein gesonderter Schriftsatz (particulars of claim, früher Statement of claim) erforderlich. Dieser kann mit der Claim form zugestellt werden, oder bis zu 14 Tage danach, spätestens jedoch vier Monate nach Ausstellung der Claim form, PD 16 para 3.2 (2).

Insofern hat die Claim form den Charakter der ursprünglichen Writs als Ladungsbefehl, nicht als schriftsätzliche Klagebegründung, beibehalten. Allerdings handelt es sich dabei durch das Erfordernis, die Klage zeitnah zu begründen, nur noch um einen formalen Aspekt.

Äußerlich ähnelt das aktuelle englische Verfahren insoweit der Klageeinleitung über das deutsche Mahnverfahren. Auch dabei bleibt die Begründung der Klage einem weiteren Schriftsatz vorbehalten. Dies gilt zwar grundsätzlich auch für die

\footnotetext{
${ }^{758} \mathrm{Ob}$ dem Gericht dann noch Schutzpflichten obliegen und ob es diese bei einer anwaltlich vertretenen Partei überhaupt ausüben darf, ist problematisch. Vgl. insofern zu § $139 \mathrm{ZPO}$ schon BGH WM 1977, S. 1201 und BGH NJW 1984, S. 310.

${ }^{759}$ Weber, ZZPInt 2000, S. 59, 73 identifiziert diverse Strukturmerkmale des Anwaltswesens und des Vorverfahrens als weitere funktionale Gegenparts zu $§ 139$ ZPO.
} 
Klage nach § 253 ZPO, da für die Zulässigkeit der Klage selbst nur die Tatsachen vorgetragen werden müssen, die den Streitgegenstand individualisieren. Praktisch hat diese Feinheit aber keine Auswirkung.

Streng gensehen entspricht das Mahnverfahren noch eher dem ursprünglichen Writ-System, da in beiden Systemen ein staatlicher Befehl an den Beklagten ergeht, den Kläger zu befriedigen, oder das streitige Verfahren aufzunehmen. Allerdings haben beide Formen der Verfahrenseinleitung gemeinsam, dass oft die bloße Existenz der Claim form bzw. des Mahnbescheides schon Druck auf den Gegner ausübt und ihn möglicherweise zur Zahlung bewegt, ohne dass eine ausführliche Klagebegründung notwendig wäre.

Obwohl die Particulars of claim nicht zwingend in die Claim form eingetragen werden müssen, gehören sie als Klagebegründung zur Klage. In ihnen muss der Kläger knapp und präzise die Tatsachen, auf die er sich beruft, darlegen. Dabei muss er Tatsachen darlegen, die ausreichen, jedes Tatbestandsmerkmal auszufüllen. Tut er dies nicht, kann das Gericht die Klage abweisen. ${ }^{760}$ In der Regel hat er aber die Möglichkeit, Ergänzungen nachzureichen. Hat der Kläger zwar Tatsachen vorgetragen, die seinen Anspruch ausfüllen, diese aber nicht ausreichend substantiiert, weist das Gericht die Klage nicht ab, da der Kläger durch weitere Substantiierung seines Vortrages die Mängel heilen kann. ${ }^{761}$ Die Anforderungen an Knappheit und Präzision sowie an die Gliederung der Schriftsätze sind erheblich höher, als im deutschen Recht. Die Pflicht zur abschnittsweisen, chronologischen und einzeln gegliederten Angabe der Tatsachenbehauptungen entspricht den positiones des römisch-kanonischen Verfahrens, wie sie auch das deutsche Recht bis zum gemeinrechtlichen Prozess kannte. In beiden Verfahren mußte der Beklagte auf die einzelnen Tatsachenbehauptungen auch dezidiert antworten oder er lief Gefahr, dass die Tatsache als zugestanden galt. Dem Gericht ist bei der Sanktionierung mangelhafter Schriftsätze ein weiteres Ermessen eingeräumt, als im deutschen Recht. Das Gericht kann die gesamte Klage abweisen, den Schriftsatz nicht zulassen, weitere Präzisierung oder Substantiierung verlangen oder einzelne Tatsachenbehauptungen ausschließen. ${ }^{762}$ Das deutsche Prozessrecht kennt nur Verspätungs- oder Kostensanktionen, z.B. bei unleserlichen oder unverständlichen Schriftsätzen, die zurückgegeben werden können ${ }^{763}$ und sodann ggf. verspätet sind, § $296 \mathrm{ZPO}$, bzw. Kostenfolgen herbeiführen, §34 GKG oder $\S 95$ ZPO. ${ }^{764}$

Wie im deutschen Verfahren dürfen die Parteien seit Einführung der CPR 1998 auch in England Rechtsausführungen machen, para. 16.3 (1) PD 16. Die Parteien sind jedoch in beiden Systemen nicht dazu verpflichtet. Unter Umständen können rechtliche Erwägungen sinnvoll sein: Zum einen, um sicher zu gehen, dass das Gericht einen Punkt nicht übersieht, zum anderen, wenn es sich um ein kompliziertes Rechtsgebiet handelt, in dem der Anwalt möglicherweise eine größere Expertise besitzt als das Gericht. Im englischen Prozess kommt hinzu, dass ein Verfahren schnell erledigt werden kann, wenn eine rechtliche Einwendung den Fall entscheiden würde und über diese Einwendung vorab entschieden wird (Preliminary point) ${ }^{765}$

\footnotetext{
${ }^{760}$ Fowler v. Lanning [1959] 1 Q.B. 426; O'Hare/Hill, Rn. 12.016

${ }^{761}$ Brophy v. Dunphys Chartered Surveyors, The Times, 11.03. 1998

${ }^{762}$ O'Hare/Hill, Rn. 12.002

${ }^{763}$ OLG Karlsruhe, NJW-RR 1987, 127

${ }^{764}$ Baumbach/Lauterbach/Albers/Hartmann, § 130 Rn. $31 \mathrm{ff}$.

${ }^{765}$ O'Hare/Hill, Rn. 12.009
} 
Die Klage soll nach deutschem Recht die Beweismittel beinhalten, derer sich der Kläger bedienen möchte, $\S 130$ Nr. 5 ZPO.

Nach englischem Recht war die Angabe von Beweismitteln im Pleading-Stadium bis zur Einführung der CPR 1998 unzulässig. Dies wurde durch para 14.3 PD 16 abgeschafft. Praktische Bedeutung hat diese Regelung allerdings bisher nicht. Es ist weiterhin übliche Praxis, in den Statements of case lediglich Tatsachenbehauptungen anzuführen, ohne Beweis anzubieten oder anzutreten.

Der Unterschied, der hier zum deutschen Recht besteht, ergibt sich aus der Zweiteilung des englischen Verfahrens in das Behauptungsverfahren (Pleadings/Statements of case) und das Beweisverfahren (Trial). In beiden Systemen dienen die Schriftsätze zwar (lediglich) der Vorbereitung der mündlichen Verhandlung, im Unterschied zum deutschen Recht ist das schriftsätzliche Verfahren in England aber mit der Eröffnung der mündlichen Verhandlung abgeschlossen.

\section{cc. In formaler Hinsicht}

Eine formal ordnungsgemäße Klage muss eigenhändig unterschrieben sein. Damit soll sichergestellt werden, dass jemand - im Anwaltsprozess ein postulationsfähiger Anwalt - Verantwortung für den Schriftsatz übernimmt.

Eine ähnliche ratio legis unterliegt dem Statement of truth gem. r. 22.1 (1) (a) CPR 1998. Anders als nach der ZPO wird der nicht mit dem Statement of truth versehene Schriftsatz allerdings als voll wirksam erachtet. Er kann lediglich keine Beweiswirkung entfalten. In anderen Sachen als der Hauptsache ${ }^{766}$ kann ein Schriftsatz, der mit dem Statement of truth versehen ist, als Beweismittel verwendet werden, r. 32.6 (2) CPR 1998. Allerdings kann das Gericht auf Antrag oder von Amts wegen die Abgabe des Statement of truth einfordern. Holt die Partei die Abgabe nicht innerhalb der gesetzten Frist nach, kann und wird das Gericht in der Regel das Statement of case für unwirksam erklären.

R. 22.1 CPR 1998 verfolgt den gleichen Regelungszweck wie das Unterschriftserfordernis nach $\S 130 \mathrm{Nr}$. 6 ZPO bzw. $\S 253$ II ZPO i.V.m. § 126 BGB. Jemand soll Verantwortung für den Schriftsatz übernehmen. Darüber hinaus zwingt r. 22.1 CPR 1998 aber auch zur wahrheitsgemäßen Tatsachendarstellung und deckt sich insoweit mit $\S 138$ I ZPO. Beide Normen gehen von einem subjektiven Wahrheitsbegriff aus. ${ }^{767}$ Die Konsequenzen, die bei Verstoß gegen die Wahrheitspflicht drohen, sind nach neuem englischen Recht ungleich schärfer als in Deutschland. Nach deutschem Recht drohen hauptsächlich prozessrechtliche Folgen, wie Kostenfolgen nach $\S 34$ GKG oder Präklusion, wenn die unwahre Behauptung zu Verzögerungen führt. Daneben haftet die unwahr vortragende Partei unter Umständen auf Schadensersatz aus $\S 826$ BGB. Strafrechtlich kann die Partei nur bei Vorliegen der weiteren Strafbarkeitsvoraussetzungen der ggf. einschlägigen Straftatbestände belangt werden. ${ }^{768}$ R. 32.14 (1) CPR 1998 statuiert hingegen eine Strafbarkeit der wissentlich unwahr vortragenden Partei wegen Contempt of court.

Darüber hinaus hat das Erfordernis der eigenhändigen Unterschrift im deutschen Recht eine Identifikations- und Verifizierungsfunktion. Diese Funktion hat in

\footnotetext{
${ }^{766}$ Hierbei kommen alle Anträge bei Gericht in Frage, unabhängig davon, ob sie ex parte ergehen. Z.B. Freezing injunctions, die Erlaubnis, die Claim Form ins Ausland zuzustellen, Zwischenstreitigkeiten über die Zustellung, Zuständigkeit etc..

${ }^{767}$ Vgl. für das deutsche Recht beispielhaft BGH MDR 1980, 214; im englischen Recht statuiert dies ausdrücklich r.32.14 (1) CPR 1998 und para. 3.8 PD 22.

${ }^{768}$ Hierbei ist insbesondere an versuchten oder vollendeten Prozessbetrug in allen Beteiligungsformen zu denken $\S \S 263,22,23,25,26,27 \mathrm{StGB}$.
} 
England nur ein Pendant, wenn die Claim form von einem Anwalt verfasst wurde. Dann muss sie von diesem auch unterschrieben werden, para 2.1 PD 5.

Bezüglich der weiteren Formalia ist das englische Recht durch eine Vielzahl an Formvorschriften zu den Schriftsätzen gekennzeichnet. So gibt para. 2.2 PD 5 die genauen Formalia der Schriftsätze vor. ${ }^{769}$ Entsprechende Formvorschriften kennt das deutsche Recht nicht; ebensowenig die allgemeine Befugnis, formal nicht ordnungsgemäße Schriftsätze nicht zuzulassen. Dies wäre auch schwerlich mit dem verfassungsrechtlich garantierten Justizgewährungsanspruch zu vereinbaren.

\section{b. Entgegenstehende Rechtshängigkeit}

Nach deutschem Recht kann ein Verfahren zwischen den gleichen Parteien über den gleichen Streitgegenstand nur einmal bei Gericht geltend gemacht werden. Hat ein Gericht schon rechtskräftig entschieden, steht der erneuten Geltendmachung desselben Anspruchs zwischen den gleichen Parteien die Rechtskraft entgegen. Hat noch kein Gericht entschieden, ist das Verfahren aber bereits rechtshängig, ist ein zweites Verfahren nach § 261 III Nr. 1 ZPO unzulässig.

Das englische Recht kennt zwar die entgegenstehende Rechtskraft (res judicata). Die entgegenstehende Rechtshängigkeit als absolutes Klagehindernis hingegen gibt es im englischen Recht nur über Art. 21 EuGVÜ/ Art. 27 EuGV-VO. Obwohl auch die englische Rechtsordnung anerkennt, dass konkurrierende Verfahren grundsätzlich problematisch und unerwünscht $\operatorname{sind}^{770}$, gibt es keinen Automatismus, der konkurrierende Verfahren verhindert. Im nationalen englischen Zivilprozess wird die entgegenstehende Rechtshängigkeit über das Institut des Verfahrensmissbrauchs (abuse of process) geregelt. ${ }^{771}$ Im internationalen Zivilverfahren, soweit es nicht vom EuGVÜ/ der EuGV-VO geregelt wird, entscheidet das Gericht die Frage, ob das eigene Verfahren eingestellt wird oder ob ein anderes Verfahren durch gerichtliche Verfügung an den Kläger verhindert wird, ${ }^{772}$ nach Forum-non-conveniens-Gesichtspunkten. ${ }^{773}$

Sowohl in internationalen, als auch in nationalen Prozessen entscheidet das Gericht nach seinem Ermessen über die Einstellung des Verfahrens.

\section{c. Besondere Verfahrensarten}

Sowohl die ZPO als auch die CPR 1998 kennen besondere Verfahrensarten, die zum Teil kein Pendant in der jeweils anderen Prozessordnung finden. Das deutsche Mahnverfahren ähnelt in seinem Aufbau dem ursprünglichen Writ-System. Es gibt jedoch in England kein eigenständiges System, einen vollstreckbaren Titel zu erwirken, ohne eine Klage anzustrengen. ${ }^{774}$

Auch das Verfahren nach part 8 CPR 1998, wonach Verfahren, bei denen ausschließlich um eine Rechtsfrage gestritten wird, oder wo ein normales Verfahren

\footnotetext{
${ }^{769}$ Siehe dazu oben B.III.2.a.bb.

${ }^{770}$ The Abidin Daver [1984] A.C. 398, 412 (H.L.); Cheshire and North, S. 347

${ }^{771}$ r. 3.4 (2) (b) CPR 1998; Bunge, Zivilprozeß, S. 93

${ }^{772}$ Vgl. zu diesen Verfügungen (Anti-Suit-Injunctions) Cheshire and North, S. 359 ff.. Die Verfügung wird nicht als direkter Eingriff in die Souveränität anderer Gerichte angesehen, da das verfügende Gericht nur seine Gewalt über den Kläger des anderen Verfahrens ausübt, in die Souveränität des anderen Gerichts somit nur mittelbar eingreift. Aufgrund dieses mittelbaren Eingriffs, der als Verletzung der Comity angesehen wird, muss das Gericht beim Erlass derartiger Verfügungen zurückhaltend und mit großer Vorsicht vorgehen; Castanho v. Brown and Root (UK) Ltd. [1981] A.C. 557 (H.L.); British Airways Board v. Laker Airways [1985] A.C. 58, 95 (H.L.).

${ }^{773}$ Vgl. zu diesem Komplex umfassend Cheshire and North, S. 334 ff.; Spiliada Maritime Corporation v. Cansulex Ltd. [1987] A.C. 460 (H.L.) als leading case.

${ }^{774}$ Umfassend zu den funktional weitgehend vergleichbaren Verfahren: Leipold S. $1 \mathrm{ff..}$
} 
unangemessen umfangreich wäre, im vereinfachten Verfahren durchgeführt werden können, kennt keinen exakten deutschen Gegenpart. Dennoch weist es gewisse Ähnlichkeiten zum Urkundenverfahren nach $\S \S 592 \mathrm{ff}$. ZPO auf. In beiden Verfahrensarten geht es dem Kläger darum, mittels eines einfachen Verfahrens möglichst schnell in den Genuss eines Titels zu gelangen. Dabei ist er in beiden Verfahren weitgehend auf schriftliche Beweismittel beschränkt. Allerdings kommen nach der ZPO nur Urkunden und Parteivernehmung in Betracht, wohingegen bei part 8 Verfahren auch schriftliche Zeugenaussagen und Sachverständigengutachten beigefügt werden können. Andere als schriftliche Beweismittel sind nur nach ausdrücklicher Genehmigung durch das Gericht zugelassen. Das Gericht bestimmt auch nach freiem Ermessen den weiteren Verlauf des Verfahrens, insbesondere, ob das Verfahren in ein normales Verfahren nach part 7 CPR 1998 übergeleitet wird. Dies geschieht auch auf Antrag des Beklagten.

Das Urkundenverfahren ist insoweit wesentlich strikter. Das Gericht hat keine Möglichkeit, andere Beweismittel als die gesetzlich vorgesehenen zuzulassen. Nur der Kläger kann den Übergang ins normale Verfahren bewirken. Der Beklagte ist auf das Nachverfahren beschränkt. Insoweit wird der Beklagtenschutz durch unterschiedliche Mechanismen bewirkt. Auch hier zeichnet sich ab, dass englischen Gerichten ein erheblich größerer Ermessensspielraum eingeräumt ist als deutschen.

\section{d. Zustellung}

Der zweite Akt nach der Einreichung der Klage ist in beiden Verfahren die Zustellung der Klage bzw. der Claim form. Dabei ist zwischen der Frage, wer für die Zustellung verantwortlich ist, und der Art der Zustellung zu differenzieren.

\section{aa. Amts- oder Parteizustellung}

Bei Einführung der RZPO 1877 war die Parteizustellung die Regel. Dementsprechend standen die Regeln über die Parteizustellung auch im Mittelpunkt des $\mathrm{Zu}$ stellungsrechts. Obwohl 1976 der Amtsbetrieb und damit auch die Zustellung von Amts wegen eingeführt wurde, blieben die Zustellungsregeln im Wesentlichen unverändert. Dieses Missverhältnis wurde durch das ZustellungsreformG vom 25.06.2001 abgeschafft und die Vorschriften über die Amtszustellung in den Vordergrund des Zustellungsrechts gerückt.

Auch in England herrschte bis ins 20. Jahrhundert als Regel die Parteizustellung vor. Mit Einführung der CPR 1998 wurde dieser Regel-Ausnahme-Mechanismus zugunsten der Amtszustellung durch das Gericht umgekehrt. Die Parteien können die Zustellung zwar immer noch selbst vornehmen, müssen dies dem Gericht aber anzeigen, damit es untätig bleibt, r. 6.3 CPR 1998. Misslingt die Zustellung durch das Gericht, wird dies dem Kläger angezeigt und er kann versuchen, das Dokument selbst zuzustellen, rr. 6.11,6.3 (1) (a) CPR 1998, para. 8.2 PD 6. Es gibt noch keine Untersuchungen dazu, wie sich das Verhältnis von Amts- zu Parteizustellung seit Einführung der CPR 1998 verändert hat. Mitarbeiter großer Anwaltssozietäten bevorzugten im Jahre 2000 weiterhin die Parteizustellung, da sie ohnehin in ständigem Geschäftsverhältnis zu Zustellungsfirmen standen, derer sie sich auch weiterhin bedienen wollten. ${ }^{775}$

Damit bleibt festzuhalten, dass in beiden Systemen grundsätzlich die Amtszustellung die Regel ist. Anders als in Deutschland, wo die Amtszustellung der Klage

\footnotetext{
${ }^{775}$ So die Aussagen diverser Mitarbeiter der Civil Litigation Departments von Baker \& McKenzie, sowie Clifford Chance in London.
} 
zwingend ist, kann in England der Kläger nach Erklärung gegenüber dem Gericht die Zustellung auch selbst vornehmen. Damit soll dem Kläger die Möglichkeit gegeben werden, eilige oder von Fristablauf bedrohte Sachen selbst zuzustellen und damit sicherzustellen, dass die Zustellung rechtzeitig erfolgt. Dieses Ziel sichert im deutschen Recht $\S 167$ n.F. ZPO (§ 270 III a.F. ZPO), wonach Fristen gewahrt werden, wenn die Zustellung ,demnächst ${ }^{6776}$ erfolgt.

\section{bb. Art der Zustellung}

Die Zustellung erfolgt in England in der Regel durch Post erster Klasse, soweit sie vom Gericht vorgenommen wird. Allerdings bestehen alternative Möglichkeiten der Zustellung durch persönliche Übergabe, Fax, DX, E-Mail oder andere elektronische Medien. Diese Varianten stehen sowohl dem Gericht, als auch den Parteien zur Verfügung. Bis auf Post und persönliche Zustellung muss sich der Beklagte oder sein Rechtsanwalt vorher mit der Zustellungsart einverstanden erklärt haben.

In besonderen Fällen kann das Gericht jede andere Zustellungsmethode anordnen, die ihm geeignet erscheint, das Schriftstück zuzustellen. Die Möglichkeiten reichen von der Zustellung an einen Bekannten oder Verwandten bis zur öffentlichen Zustellung über Zeitungsanzeigen.

Das deutsche Recht räumt dem Zustellenden weniger Ermessen bei der Wahl der Mittel ein, als das englische Recht. Die Hauptformen sind in beiden Systemen noch die persönliche Zustellung, sei es durch einen Gerichtsvollzieher in Deutschland oder einen Zusteller in England, und die Zustellung durch die Post. Dabei ist in England Zustellung per Post erster Klasse die Regel. In Deutschland steht zu erwarten, dass die Zustellung durch Einschreiben mit Rückschein an Bedeutung gewinnt. Ob diese Art der Zustellung in England möglich ist, bleibt abzuwarten. Von zukunftsweisender Bedeutung sind in beiden Ländern die neuen Vorschriften zur Zustellung mit modernen Telekommunikationsmedien:

Die Zustellung per Fax ist in Deutschland gegen Empfangsbekenntnis an Anwälte und entsprechende Personen nach $\S 174$ II ZPO erlaubt. In England muss der Anwalt mit der Zustellung per Telefax einverstanden sein. Dieses Einverständnis wird jedoch vermutet, so dass die Regelungen in beiden Jurisdiktionen einander entsprechen. Ähnliches gilt auch für die Zustellung per E-Mail. Allerdings geht die deutsche Regelung hier weiter als die englische. In Deutschland ist die Zustellung per E-Mail an Anwälte etc. grundsätzlich möglich; an andere Verfahrensbeteiligte nur, wenn diese sich mit der Zustellung per E-Mail einverstanden erklärt haben, § 174 III ZPO. In England ist die Zustellung per E-Mail nur möglich, wenn beide Parteien anwaltlich vertreten sind, die Zustellung an den Anwalt erfolgt und sich der Anwalt im Vorfeld mit der elektronischen Zustellung einverstanden erklärt hat.

Nach englischem Recht kann die Tatsache, dass das Dokument nicht in Papierform nachgereicht wird, bei einem Streit über die Zustellung vom Gericht in Betracht gezogen werden. Dazu gibt es kein deutsches Pendant, so dass zu erwarten ist, dass in Deutschland, anders als in England, nach einmal erfolgter Zustellung per E-Mail keine verkörperte Kopie nachgesandt werden wird.

Die CPR 1998 stellen für jede der gesetzlich aufgeführten Zustellungsformen eine gesetzliche Vermutung für einen Tag auf, an dem die Zustellung als erfolgt gilt, r 6.7 (1) CPR 1998.

\footnotetext{
${ }^{776}$ Zum Begriff s.o. C.VI.2.a
} 
Das deutsche Recht kommt ohne solche Vermutungen aus. Das Zustellungsdatum entspricht dem tatsächlichen Zugang beim Empfänger oder bei der Ersatzzustellung durch Niederlegung dem Zugang der Benachrichtigung. Nur bei der öffentlichen Zustellung gilt ein fingiertes Datum.

Dieser Unterschied zum englischen Recht ist eine Folge der unterschiedlichen materiellen Rechtslage bei der Übermittlung von Willenserklärungen. Während im deutschen Recht auf den Zugang abgestellt wird, entscheidet in England grundsätzlich schon die Abgabe der Willenserklärung. ${ }^{777}$ Dies setzt sich auch bei der Zustellung fort. In England ist prinzipiell die Abgabe des zuzustellenden Schriftsatzes entscheidend. Zum Schutz des Empfängers wird die Wirkung aber auf den gesetzlich vermuteten Zeitpunkt verschoben. In Deutschland hingegen ist der Zeitpunkt des Zugangs entscheidend, so dass ein Empfängerschutz nicht erforderlich ist, da es dem Empfänger obliegt, seinen Herrschaftsbereich zu organisieren.

Wer die Zustellung bewusst vereitelt, indem er die Annahme verweigert, muss die Zustellung in beiden Systemen gegen sich gelten lassen. Im deutschen Recht folgt dies aus $\S 179 \mathrm{ZPO}$, im englischen aus Barclays Bank of Swaziland Ltd. v. $H a h n^{778}$. Gleiches muss auch für andere vorsätzliche Zugangsvereitelungen gelten. ${ }^{779}$

Bei der Zustellung ins Ausland muss im Wesentlichen zwischen zwei verschiedenen Szenarien unterschieden werden:

1. Erfolgt die Zustellung an einen Beklagten im EU-Ausland, sind die Regeln der Verordnung über die Zustellung gerichtlicher und außergerichtlicher Schriftstücke in Zivil- oder Handelssachen (EuZustVO) anwendbar. Dabei wird in Deutschland Art. 14 EuZustVO dahingehend modifiziert, dass durch die Post nur per Einschreiben mit Rückschein zugestellt werden darf. Bei der Zustellung durch die Post handelt es sich um eine gleichwertige Zustellungsmethode neben den anderen angeführten Varianten. Insbesondere ist die Zustellung nicht subsidiär zum dezentralen direkten Behördenverkehr. ${ }^{780} \mathrm{Da}$ in Bezug auf Zustellungen im EU-Ausland in England und Deutschland die gleiche Verordnung gilt, erübrigt sich ein Vergleich.

2. Erfolgt die Zustellung in ein anderes Land, kann entweder nach dem HZÜ zugestellt werden, das sowohl in Deutschland, als auch in England gilt, oder soweit Staatsverträge nicht anwendbar sind, nach den nationalen Vorschriften.

Dabei ergeben sich erhebliche Unterschiede zwischen deutschem und englischem Recht. Während in Deutschland nach § 183 I ZPO über Rechtshilfe (Nr. 2) oder per Einschreiben mit Rückschein (Nr. 1) zugestellt werden darf, ist eine Zustellung ins Ausland nach englischem Recht nur sehr eingeschränkt möglich. R. 6.20 CPR 1998 legt im Einzelnen fest, wann eine Claim form ausnahmsweise mit Erlaubnis des Gerichts im Ausland zugestellt werden darf. Die einzelnen Varianten, unter denen das Gericht nach freiem Ermessen die Erlaubnis erteilen darf, beschreiben Situationen, in denen ein enger Sachzusammenhang zwischen England und dem streitigen Sachverhalt oder den Parteien besteht. ${ }^{781}$ Dabei muss die

\footnotetext{
${ }_{777}$ Vgl. Zweigert/Kötz, S. 352 f.; 356

${ }^{778}$ [1989] 1 W.L.R. 506 (H.L.) noch zur alten Rechtslage nach RSC O. 10 r. 1 (3) (a). Dabei handelte es ich nicht um eine Annahmeweigerung im engeren Sinne, sondern um die Vereitelung des Zugangs durch bewusstes Fernbleiben von zu Hause, in Kenntnis, dass ein Writ abgegeben worden war.

${ }^{779}$ Insoweit ist $\S 179 \mathrm{ZPO}$ analog anzuwenden.

${ }^{780}$ So auch Lindacher, ZZP 114 (2001), S. 179, 185, aA Heß, JZ 2001, S. 573, 577, ders. NJW 2001, S. 15,19

${ }^{781}$ Siehe im Einzelnen dazu r. 6.20 (1-18) CPR 1998.
} 
Erlaubnis des Gerichts unter Darlegung aller erheblichen Umstände beantragt und die Umstände müssen eidesstattlich versichert oder durch Urkunden bewiesen werden. Die Erlaubnis wird vom Gericht nur erteilt, wenn es sich nach eingehender Prüfung für das forum conveniens hält.

Der Unterschied zum deutschen Recht ergibt sich aus folgender Besonderheit:

Nach englischem Recht ist mit der Zustellung der Claim form in der Regel auch die internationale Zuständigkeit der englischen Gerichte etabliert. Die Prüfung der internationalen Zuständigkeit wird demnach auf das Antragsverfahren, in dem die Erlaubnis zur Zustellung ins Ausland gegeben oder verweigert wird, verlagert. Dies folgt aus dem zurückhaltenden Verhalten englischer Gerichte anderen Staaten gegenüber. Die Zustellung ins Ausland wird immer auch als nicht erwünschter Eingriff in die Souveränität des anderen Staates und damit als Verletzung der Comity empfunden. ${ }^{782}$

Im deutschen Recht findet die Prüfung der Zuständigkeit erst im Verfahren selbst statt. Dabei wird der Comitas dadurch Rechnung getragen, dass die internationale Zuständigkeit deutscher Gerichte von Amts wegen zu prüfen ist. ${ }^{783}$

\section{cc. Zuzustellende Dokumente}

In beiden Systemen ist dem Beklagten die Klage inklusive ihrer Begründung zuzustellen. Mit der Klage bzw. der Claim form/Particulars of claim werden dem Beklagten auch die gerichtlichen Verfügungen bzw. gesetzlichen Fristen mitgeteilt. $\mathrm{Zu}$ diesem Zweck gibt es im englischen Recht einheitliche Formulare und feste Automatismen. ${ }^{784}$ Der Beklagte muss seine Verteidigungsbereitschaft innerhalb von zwei Wochen nach Zustellung anzeigen. Dies tut er entweder durch die Verteidigung selbst oder durch Abgabe der Bestätigung der Zustellung. Im zweiten Fall hat er 28 Tage für seine Klageerwiderung.

Im deutschen Recht steht das Verfahren etwas mehr im Ermessen des Gerichts, da es einen frühen ersten Termin bestimmen kann. Tut es dies nicht, entsprechen die deutschen Regeln den englischen erstaunlich genau. Auch nach der ZPO beträgt die Frist zur Erklärung der Verteidigungsbereitschaft zwei Wochen. Die Klageerwiderung selbst muss binnen weiterer zwei Wochen (oder mehr, wenn das Gericht es für angemessen hält) eingereicht werden. Nach dem gesetzlichen Leitbild muss also der Beklagte in beiden Systemen innerhalb von zwei Wochen seine Verteidigungsbereitschaft erklären und innerhalb von vier Wochen auf die Klage erwidern. Das englische Verfahren ist lediglich formalisierter und bietet derzeit dem Gericht keinen Ermessensspielraum. Ein dem frühen ersten Termin entsprechendes Verfahren sehen die CPR 1998 selbst nicht vor. Allerdings kann nach r. 7.9 CPR 1998 eine Practice Direction erlassen werden, die bestimmt, wann das Gericht einen Termin zur mündlichen Verhandlung schon mit Ausstellung der Claim form festsetzen kann und was für ein Verfahren in solchen Fällen anzuwenden ist, r. 7.9 (a und c) CPR 1998.

\section{e. Gesellschaftspolitische Gründe für die zivilprozessualen Veränderungen}

Die Zivilprozessordnungen Englands und Deutschlands, wie sie das 20. Jahrhundert beherrschten, waren Kinder unterschiedlicher Gesellschaften und Entwicklungen. Das englische Zivilverfahren hatte sich in jahrhundertelanger ruhiger Auseinandersetzung der politischen Gruppen einer traditionellen Gesell-

\footnotetext{
${ }^{782}$ Cheshire and North, S. $318 \mathrm{f}$.

${ }^{783}$ BGH NJW 1996, S. 1412; Baumbach/Lauterbach/Albers/Hartmann, Übers $§ 12$, Rn. 8

${ }^{784}$ S.o. B.III.3.c.
} 
schaft unter vermittelnder Mitwirkung der Krone entwickelt. ${ }^{785}$ Als solches war es gewachsenes Recht, das nur durch gesetzgeberische Korrektureingriffe gelenkt und den gesellschaftlichen Veränderungen angepasst wurde.

Die deutsche ZPO hingegen stellt vorerst den Endpunkt einer Prozessrechtsgeschichte dar, in der zum Teil völlig verschiedene Verfahren miteinander konkurrierten, einander beeinflussten oder ablösten. Dabei spielte die gesellschaftliche Entwicklung, die auf dem Festland stürmischer war als auf der Insel, eine ebenso große Rolle, wie die territorialen Einflüsse, die wiederholt zu einer Beeinflussung des Rechts „von außen“ führten.

Obwohl die ZPO in ihrer wesentlichen Struktur unverändert geblieben ist, lassen sich an ihrer Entwicklung Veränderungen in der Gesellschaft ablesen, insbesonder an einigen Punkten im Verfahren. ${ }^{786}$

Die RZPO von 1877 ging noch vom Parteibetrieb aus. Das Verfahren sollte ausschließlich in den Händen der Parteien ruhen. ${ }^{787}$ Insbesondere die Zustellungsnormen gingen von der Parteizustellung als Regel aus. Der Einfluss sozialistischen Denkens machte sich aber bereits Anfang des 20. Jahrhunderts bemerkbar, und es kam zu Einschränkungen des Parteibetriebs, indem durch die Novelle von 1909 am Amtsgericht der Amtsbetrieb eingeführt wurde. ${ }^{788} \mathrm{Im}$ dritten Reich, als in Deutschland wieder eine autoritäre Staatsform Einzug hielt, wurde der Parteibetrieb an allen Gerichten zugunsten des Amtsbetriebs abgeschafft. Die Eigenverantwortlichkeit des Bürgers gegenüber dem Staat, eine Grundidee des Liberalismus, aus dem heraus die ZPO entstanden war, war im Nationalsozialismus nicht erwünscht. Die Abschaffung des Parteibetriebs und damit Schwächung der Dispositionsmaxime entsprach der Vorstellung, die die Nationalsozialisten vom Verhältnis Staat-Bürger hatten.

Als nach dem Krieg das Recht auf den Stand von 1933 zurückversetzt wurde, führte man auch den Parteibetrieb wieder ein. $\mathrm{Zu}$ dieser restaurativen Tendenz ${ }^{789}$ kam es, weil das dritte Reich als abschreckendes Beispiel für einen „starken“ Staat frisch in der Erinnerung des bundesdeutschen Gesetzgebers war. Erst 1976 entschied man sich dann doch zugunsten des Amtsbetriebs. Diese Entscheidung, die dem Staat mehr Verantwortung überträgt, diente zum einen dem Wunsch nach Beschleunigung, zum anderen ist sie aber signifikant für eine Entwicklung, die sich seit den siebziger Jahren verstärkt abzeichnet: Der Bürger ist als Verbraucher schutzbedürftig und hat dem Sozialstaat gegenüber einen Anspruch auf diesen Schutz. Im Rahmen dieser Entwicklung wurden und werden dem Staat zunehmend Fürsorgepflichten übertragen. Mit der Entwicklung vom bürgerlichen Rechtsstaat des 19. Jahrhunderts hin zum Sozialstaat veränderten sich auch die Ansprüche der Gesellschaft an den Staat. Nach der Grundidee der ZPO sollte der Staat größtmögliche Freiheit garantieren, also den Prozess so weit wie möglich in die Hände der Parteien legen. Nach dem heutigen, sozialstaatlich geprägten Verständnis hat der Staat einen sozial gerechten Ausgleich zwischen seinen Bürgern vorzunehmen. Insoweit ist er notwendig auch Interventionsstaat. ${ }^{790}$ Diese Sozial-

\footnotetext{
${ }^{785}$ Wieacker, S. 498

${ }^{786}$ Zur Bedeutung der Mündlichkeit vgl. Dreymüller, Zeugenbeweis, S. 119 ff. Ob sich die deutsche Prais extensiver Bezugnahme auf Schriftsätze damit begründen lässt, dass das deutsche Recht nicht mit der Mündlichkeit gewachsen sei (Dreymüller, ZVglRWiss 101 (2002), S. 471, 474), ist zumindest fraglich.

${ }^{787}$ Wassermann, S. 38

${ }^{788}$ Wassermann, S. 66

${ }^{789}$ Wassermann, S. 62

${ }^{790}$ Ipsen, Rn. 969
} 
staatsbindung des Gerichts ${ }^{791}$ gilt auch für das Zivilverfahren, das zunehmend wieder in die Hände des Gerichts gelegt wird.

Deutlicher als am Amtsbetrieb wird dies in der Entwicklung der materiellen Prozessleitung, § 139 ZPO. Diese Norm, die zur Sachverhaltsaufklärung eingeführt worden war, entwickelte sich zunehmend zur Ausprägung des Sozialstaatsprinzips und verpflichtet den Richter heute zur aktiven Fürsorge den Parteien gegenüber, selbst wenn diese anwaltlich vertreten sind. Der Zivilprozess entwickelt sich demnach in Richtung einer Kooperationsmaxime und macht die Beteiligten zu einer Art prozessualen Arbeitsgemeinschaft. ${ }^{792}$ Auch die Einführung der $\S \S 175,176$ ZPO, die Neufassung der Prozessförderungspflichten der Parteien und die Ausweitung der Präklusionsvorschriften im Jahre 1979 stellen Entwicklungen in diese Richtung dar, da sie alle die Befugnisse des Gerichts stärken und die der Parteien beschneiden. Das gilt auch für die Beschleunigungsregeln. Die oft beklagte lange Prozessdauer liegt häufig nicht am Gericht. Würden die Parteien in Klage und Klageerwiderung den tatsächlichen Prozessstoff umfassend aufklären, könnte das Gericht in der Regel mit einem weiteren Beweistermin zur Entscheidung gelangen. Nach liberaler Konzeption wären Beschleunigungsvorschriften überflüssig, sogar kontraproduktiv, da sie den Parteien einen Teil der Verfahrensherrschaft entziehen.

Die sozialstaatliche Entwicklung als Gegengewicht zur kapitalistischen Wirtschaftsordnung zeichnet sich in jüngerer Vergangenheit ganz besonders in den europäischen Verbraucherschutzgesetzen ab. Über diese Gesetze und aufgrund der Tatsache, dass sich die wirtschaftlichen und sozialen Verhältnisse in den westeuropäischen Staaten zunehmend angleichen, hat ein verstärkt sozialstaatliches Verständnis in der englischen Justiz Einzug gehalten. Das zeigt sich auch an der starken Zunahme von Kodifikationen durch das englische Parlament. Insbesondere soziale Wertsetzungen sind ohne den Gesetzesstaat kaum durchzusetzen. ${ }^{793}$ Die CPR 1998 sind ein Ergebnis dieser Entwicklungen. Das wird aus zwei Gründen an den CPR 1998 besonders deutlich:

Erstens handelt es sich um ein ganz neues Regelwerk, so dass die Unterschiede zum Vorgängersystem klarer sind, als bei bloßen Änderungen eines bestehenden Systems. Zweitens hatte im englischen Zivilprozess ein liberaler Grundgedanke in sehr reiner Form Bestand. Das ganze Verfahren war seit jeher viel adversativer als die Verfahren auf dem Festland.

Das neue englische Zivilverfahren bestätigt die Beobachtungen, die anhand der ZPO gemacht wurden auch für England und legt damit eine gesamteuropäische Gesellschaftsentwicklung nahe.

Mit Einführung der CPR 1998 wurden dem Gericht erstmalig weitreichende Prozessleitungsrechte und -pflichten eingeräumt. ${ }^{794}$ Im Rahmen der Tracks bestimmt das Gericht jetzt auch selbständig über sein weiteres Verfahren, was insoweit mit $\S \S 175,176$ ZPO vergleichbar ist. Die CPR 1998 räumen dem Gericht weitreichende Sanktionsmöglichkeiten zur Disziplinierung der Parteien von Amts wegen ein, und mit der Möglichkeit, über den Antrag hinaus zu urteilen, wurde die Dispositionsmaxime zugunsten der „Parteienfürsorge“ eingeschränkt. Auch die Abkehr von der Parteizustellung als Regelfall kennzeichnet diese Entwicklung. Schließlich zeigt das Statement of truth, dass die Parteien nunmehr dem Gericht gegenüber verantwortlich sein sollen, nicht mehr dem Gegner.

\footnotetext{
${ }^{791}$ Wassermann, S. 76

${ }^{792}$ Wassermann, S. 86, ausführlich auf S, 97 ff., $110 \mathrm{ff}$.

${ }^{793}$ Wieacker, S. $499 \mathrm{f}$.

${ }^{794}$ Siehe dazu oben B.III.1
} 
Abschließend ist in beiden Ländern festzustellen, dass sich die Prozessordnungen der modernen Kommunikations- und Informationsgesellschaft anpassen und sich für neue Medien öffnen.

Bei Betrachtung der gesellschaftlichen Entwicklung im Europa des späten 20. und frühen 21. Jahrhunderts stellen sich die auffälligen Gemeinsamkeiten zwischen dem deutschen und englischen Verfahren der Gegenwart als nicht so erstaunlich dar, wie die unterschiedlichen Rechtstraditionen dies vermuten ließen. 


\section{Aktuelle Entwürfe für ein neues Zivilprozessrecht im Rechtsvergleich}

Die steigende Zahl transnationaler Geschäftsbeziehungen und daraus folgend auch transnationaler Prozesse hat in den letzten Jahren die Diskussion um länderübergreifende Zivilprozessordnungen angefacht. Dabei werden im Wesentlichen drei Konzepte diskutiert. ${ }^{795}$

\section{Storme- Entwurf}

Der Vorschlag der Kommission für ein europäisches Zivilprozessgesetzbuch (Storme- Entwurf) verfolgt das Kozept einer einheitlichen Zivilprozessordnung. Der Vorschlag war als Modellgesetz gedacht. Aber seit mit dem EG-Vertrag von Amsterdam die Kompetenz für den internationalen Zivilprozess auf europäische Ebene verlagert wurde, ${ }^{796}$ scheint ein einheitliches Zivilprozessrecht für Europa ein geraumes Stück näher gerückt zu sein. ${ }^{797}$

Das Modellgesetz der Kommission für ein europäisches Zivilprozessrecht entstand von 1987 bis 1993. Jedes der zwölf Mitglieder der Kommission erstellte als Berichterstatter für einen Abschnitt eine rechtsvergleichende Analyse, auf deren Basis dann die konkreten Vorschläge erstellt wurden. ${ }^{798}$ Dabei wurden verschiedene Regelungsbereiche, die besonders national geprägt sind, von vornherein ausgenommen. ${ }^{799}$ Aber auch von diesen Lücken abgesehen, bleibt der Entwurf oft fragmentarisch. ${ }^{800}$

\section{Die Verfahrenseinleitung}

Die Regelungen zur Verfahrenseinleitung finden sich in Art. 2 des Entwurfs. Sie umfassen allgemeine Regeln zum System der Verfahrenseinleitung, Art. 2.1.1, Zustellungsvorschriften, Art. 2.1.2, Bestimmungen zum Inhalt des einleitenden Schriftstücks, Art. 2.1.3 sowie Regeln zur Verjährungsunterbrechung, Art. 2.2.1 und zur entgegenstehenden Rechtshängigkeit, Art. 2.2.2.

Darüber hinaus regelt Art. 11 die Verfahrenseinleitung über das Mahnverfahren.

\section{a. Allgemeines Verfahren}

Das Grundkonzept des Entwurfs geht davon aus, dass das Verfahren durch Einreichung eines Schriftstückes bei Gericht begonnen wird. Diesem Schriftstück wird sodann durch eine gerichtliche Handlung hoheitlicher Charakter verliehen.

Allerdings stellt dieses Verfahren im Entwurf nur ein anzustrebendes Ziel an. Dementsprechend sind einige der im Folgenden dargestellten Regeln auf andere Einleitungsverfahren abgestimmt.

Nach Art. 2.1.1.1 darf das einleitende Schriftstück keinen Zweifel an seiner offiziellen Natur lassen und muss die Richtigkeit der darin enthaltenen formalen Verfügungen $^{801}$ gewährleisten. Im Schriftstück selbst muss auch schon die erste

\footnotetext{
${ }^{795}$ Stürner, Europäischer Zivilprozeß, S. 2 ff. identifiziert fünf Grundmodelle, bedient sich dabei aber anderer Differenzierungskriterien.

${ }^{796}$ Art. 65 EG

${ }^{797} \mathrm{He}$, NJW 2000, S. 23, 27

${ }^{798}$ Storme, RabelsZ 56 (1992), S. 290, 298

${ }^{799}$ Dabei handelt es sich insb. um die Gerichtsverfassung, die Rechtskraft und die Prozessmaximen; vgl. Lemken, ZZP 109 (1996), S. 337.

${ }^{800}$ Vgl. dazu unten z.B. die Lücken bei den objektiven Anforderungen an das einleitende Schriftstück. Vgl. auch Storme, Rapprochement, S. 69.

${ }^{801}$ Dabei handelt es sich um das Ausstellungsdatum und die Einlassungs- oder Erscheinungsfristen.
} 
Terminsbestimmung durch Gesetz oder das Gericht enthalten sein, Art. 2.1.1.2. Mit Einreichung des Schriftstückes bei Gericht ist die Sache rechtshängig im Sinne der Einrede entgegenstehender Rechtshängigkeit, Art. 2.1.1.2. Allerdings ist $\mathrm{zu}$ beachten, dass es sich dabei nur um eine Sollvorschrift handelt, was aber in Anbetracht des Charakters als Diskussionsentwurf eher in den Hintergrund tritt.

Soweit nationales Recht es vorsieht, kann ein Verfahren auch durch gemeinsames Anrufen des Gerichts durch die Parteien begonnen werden, Art. 2.1.1.3.

\section{b. Inhaltliche Anforderungen an die Klageschrift}

Inhaltlich muss das einleitende Schriftstück einen Antrag enthalten, Art. 2.1.3.1 des Entwurfs, und soll, soweit es vernünftigerweise praktikabel (reasonably practicable) ist, die Tatsachen angeben, auf denen der Anspruch beruht, und die dazugehörigen Beweismittel, Art. 2.1.3.1. Dem Wortlaut der Norm ist nicht zu entnehmen, ob der Kläger nur Tatsachen angeben muss, die ausreichen, um den Streitgegenstand $\mathrm{zu}$ individualisieren, oder ob er bereits in der Klageschrift schlüssig und substantiiert vortragen muss. Die Motive allerdings stützen die erste Ansicht, da sie von einer starren Festlegung des Klägers auf den Zeitpunkt der Klageerhebung gerade absehen. ${ }^{802}$ Es soll dem Kläger auch möglich sein, Tatsachen bis zur Klageerwiderung zurückzuhalten. ${ }^{803}$ Dies soll sich aus der Praktikabilitätsklausel ergeben ${ }^{804}$ Rechtsansichten dürfen, müssen aber nicht angeführt werden ${ }^{805}$ Beweismittel sollen schon in der Klage angegeben werden. Da auch sie der Praktikabilitätsklausel unterliegen, bleibt es fraglich, in welchem Fall und zu welchem Zeitpunkt sie angegeben werden müssen. Die Tatsache, dass Mängel auf richterliche Anordnung hin behoben werden können ${ }^{806}$, spricht dafür, dass die Vorschrift eher lax zu handhaben ist.

Nach Art. 2.1.3.3 des Entwurfs muss die Klageschrift in Anwaltssachen Namen und Adresse des Anwalts angeben. Ist die anwaltliche Vertretung nicht Pflicht, bedient sich die Partei aber dennoch eines Rechtsbeistandes, ist dessen Name und Adresse anzuführen. ${ }^{807}$ Ein Kläger mit Wohnsitz im Ausland muss eine inländische Zustellungsadresse angeben, Art. 2.1.3.4 des Entwurfs. Bei Parteienmehrheit kann durch nationales Recht festgelegt werden, dass die Parteien eine gemeinsame Zustellungsadresse angeben müssen, Art. 2.1.3.5.

Nach Art. 2.1.3.2 des Entwurfs ist der Kläger mit der Klageschrift darüber zu informieren, was er zu tun hat und welche Folgen bei Säumnis drohen.

Weitere Angaben sieht der Entwurf nicht vor. Insbesondere fehlen Angaben zu den Parteien und zum Gericht, inwiefern Klagen beziffert werden müssen und ob die Klage unterschrieben werden muss.

\section{c. Zustellung}

Die Zustellungsnormen des Entwurfs belassen das Zustellungsrecht grundsätzlich in den Händen des nationalen Gesetzgebers. ${ }^{808}$ Soweit sie die Zustellung regeln, bleibt dies fragmentarisch. Der Grundsatz ist die persönliche Zustellung an den Beklagten durch den Kläger, Art. 2.1.2.1. I. An juristische Personen wird durch Zustellung an den gesetzlichen Vertreter oder einen Empfangsbevollmächtigten

\footnotetext{
${ }^{802}$ Storme, Rapprochement, S. 81

${ }^{803}$ Storme, Rapprochement, S. 81

${ }^{804}$ Schelo, S. 107

${ }^{805}$ Es gilt der iura-novit-curia-Grundsatz; Roth, ZZP 109 (1996), S. 271, 284

${ }^{806}$ Storme, Rapprochement, S. 82

${ }^{807}$ Dies soll der Korrespondenz der Parteivertreter dienen; Storme, Rapprochement, S. 123

${ }^{808}$ Storme, Rapprochement, S. 78
} 
zugestellt, Art. 2.1.2.1 II. Kann die persönliche Zustellung nicht bewirkt werden, muss der Kläger dem Gericht genau darlegen, was er im Einzelnen versucht hat, um die Klage zuzustellen, Art. 2.1.2.2 II des Entwurfs. Dabei handelt es sich um eine Beweisvorschrift, deren Rechtsfolgen das nationale Recht regeln soll. ${ }^{809}$ Art. 2.1.2.3 des Entwurfs verpflichtet die Verwaltungsbehörden und Sozialversicherungsträger solcher Länder, in denen kein verlässliches Meldewesen besteht, dem Kläger Auskunft zu erteilen, sofern kein Geheimnisschutz entgegensteht. Erst wenn alle Bemühungen des Klägers fruchtlos verlaufen sind, kann das Gericht die Ersatzzustellung oder entsprechende Maßnahmen nach nationalem Recht anordnen, Art. 2.1.2.4 II. Damit legt der Entwurf besonderen Wert auf die Subsidiarität der Ersatzzustellung, was vor dem Hintergrund rechtlichen Gehörs verständlich ist. ${ }^{810}$ Die Praktikabilität der Regelungen muss jedoch in Zweifel gezogen werden. In den meisten europäischen Ländern ist die Ersatzzustellung ein rechtlich subsidiäres Mittel, tatsächlich jedoch die Regel. ${ }^{811}$

\section{d. Verjährungsunterbrechung und lis alibi pendens}

Da die Harmonisierung des Einleitungsverfahrens nur als angestrebtes Ziel definiert wird, beziehen sich die Vorschriften über die Verjährungsunterbrechung und die entgegenstehende Rechtshängigkeit auf die verschiedenen in den Mitgliedsstaaten herrschenden Konzepte der Verfahrenseinleitung.

Handelt es sich um ein Verfahren, in dem das einleitende Schriftstück erst dem Gericht und anschließend dem Beklagten zugeleitet wird, entscheidet der Zeitpunkt des Eingangs bei Gericht über die Unterbrechung der Verjährung, Art. 2.2.1.1, und die Rechtshängigkeit im Sinne des lis alibi pendens, Art. 2.2.2.1. Beide Effekte entfallen jedoch, wenn die Klageschrift dem Beklagten nicht innerhalb von drei Monaten zugestellt wird.

Bei Verfahren, in denen die Klageschrift zuerst dem Beklagten zugestellt wird, wird die Verjährung mit Zustellung an den Beklagten unterbrochen, Art. 2.2.1.2, und die Rechtshängigkeit im Sinne des lis alibi pendens bewirkt, Art. 2.2.2.2. Für das lis alibi pendens gilt dies aber nur unter der Bedingung, dass die Klage auch innerhalb der in den nationalen Gesetzen vorgesehenen Frist bei Gericht eingereicht wird, Art. 2.2.2.3. Ob es sich dabei um eine aufschiebende ${ }^{812}$ oder eine auflösende ${ }^{813}$ Bedingung handelt, bleibt unklar ${ }^{814}$ Erstaunlich ist, dass die Bedingung für die Verjährungsunterbrechung nicht formuliert wurde, obwohl es nach der Kommentierung nicht wünschenswert ist, dass die Verjährung unterbrochen wird, wenn die Klage nicht vor Gericht verhandelt wird. ${ }^{815}$ Insoweit bietet es sich an, Art 2.2.2.2 des Entwurfs auf die Verjährungsvorschriften analog anzuwenden. ${ }^{816}$

Die Unterbrechung der Verjährung tritt auch ein, wenn der Kläger das falsche Gericht anruft, Art. 2.2.1.3, einstweiligen Rechtsschutz begehrt, Art. 2.2.1.4, den Anspruch im Mahnverfahren geltend macht, Art. 2.2.1.5, oder ein Schlichtungsverfahren durchgeführt wird, Art. 2.2.1.6. Die Verjährungsunterbrechung fällt hingegen in allen Fällen weg, wenn nicht innerhalb von drei Monaten auf die Ent-

${ }^{809}$ Roth, ZZP 109 (1996), S. 271, 285

${ }^{810}$ Bei der Ersatzzustellung besteht immer das Risiko, dass der Empfänger nicht oder nicht zeitnah in den Besitz des Schriftstüks gelangt.

${ }^{811}$ Roth, ZZP 109 (1996), S. 271, 285

${ }^{812}$ Das legt der Wortlaut der englischen Fassung nahe.

${ }^{813}$ Dafür spricht die französische Fassung.

${ }^{814}$ Ein Fallbeispiel, bei dem dieser Unterschied relevant werden kann, findet sich bei Schelo,

S. 110

${ }^{815}$ Storme, Rapprochement, S. 124

${ }^{816}$ Schelo, S. 109 spricht sich für eine analoge Anwendung des Art. 2.2.1.1 S. 2 des Entwurfs aus. 
scheidung des Gerichts hin, das Verfahren am zuständigen Gericht anhängig gemacht, das Hauptsacheverfahren angestrengt, der Mahnbescheid zugestellt oder das streitige Verfahren anhängig gemacht wird.

Die Folgen der Rechtshängigkeit richten sich nach Art 2.2.2.3 des Entwurfes. Wird ein Verfahren zwischen den gleichen Parteien über den gleichen Gegenstand bei verschiedenen Gerichten rechtshängig, setzt das zweitangerufene Gericht das Verfahren aus, bis das erstangerufene über seine Zuständigkeit entschieden hat. Erklärt sich letzteres Gericht für zuständig, muss das vorgenannte die Klage abweisen. ${ }^{817}$ Leider gibt der Entwurf keinen Aufschluss darüber, wann es sich um den gleichen Gegenstand handelt.

\section{e. Einleitung im Mahnverfahren}

Das Mahnverfahren (Order for payment) ist in Art. 11 geregelt. Es ist nur bei Zahlungsklagen möglich. Der Antrag muss folgenden Inhalt haben:

Die Namen der Parteien und des Gerichts, die genaue Angabe des Antragsgegenstands und die Versicherung, dass der Gläubiger keine Gegenleistung schuldet, Art. 11.2 Nr. 1. Ferner muss der Antragsteller seinen Antrag sowie die Zinsen und Nebenforderungen separat summarisch begründen, Art. 11.2 Nr. 3, damit das Gericht den Anspruch auf seine Schlüssigkeit hin prüfen kann. ${ }^{818}$

Die Partei oder ihr Vertreter müssen den Antrag unterschreiben und ihm zumindest in Kopie alle Urkunden beifügen, die geeignet sind, den Anspruch nach Grund und Höhe zu beweisen, Art. 11.2 Nr. 3. ${ }^{819}$ Damit hat sich die Kommission für ein Urkunden-Mahnverfahren entschieden, was dazu führt, dass Ansprüche, die nicht durch Urkunden bewiesen werden können, z.B. weil solche nicht existieren, im Mahnverfahren nicht geltend gemacht werden können. Dies gilt selbst dann, wenn die Forderung unbestritten ist. Obwohl das Bedürfnis, die Entscheidungen im Mahnverfahren durch ein „kleines Beweisverfahren“820 abzusichern, verständlich ist, bleibt dem Kläger in einer Vielzahl von Situationen das kostengünstige und schnelle Mahnverfahren verwehrt.

Der Antrag ist auf einem vorgegebenen Formular einzutragen, Art. 11.3. Dadurch soll die Einheitlichkeit gewahrt und die Bearbeitung im elektronischen Rechtsverkehr vorbereitet werden. ${ }^{821}$

Nach Einreichung des Antrags wird dieser bei Gericht auf seine Zulässigkeit und Schlüssigkeit hin geprüft. Ob diese Prüfung von einem Richter ${ }^{822}$ oder einem Rechtspfleger ${ }^{823}$ durchgeführt werden muss bzw. kann, bleibt offen. Ist der Antrag unzulässig oder unbegründet, wird er abgelehnt. Die Ablehnung erwächst nicht in Rechtskraft und ist nicht anfechtbar, Art. 11.4 I. Wenn das Gericht einen Mahnbescheid erlässt, wird er dem Schuldner zusammen mit einer eindringlichen Rechtsbehelfsbelehrung zugestellt, Art. 11.5 II 2. Dieser muss daraufhin die Summe innerhalb von acht Tagen zahlen, sonst werden ihm die Kosten des Verfahrens auferlegt, Art. 11.5 II, ${ }^{824}$ oder binnen zwei Wochen Widerspruch auf einem beigefüg-

\footnotetext{
${ }^{817}$ Die Regelung entspricht Art 21 EuGVÜ.

${ }^{818}$ Schilken, ZZP 109 (1996), S. 315, 319 und 320

${ }^{819}$ Kritisch dazu Schilken, ZZP 109 (1996), S. 315, 322.

${ }^{820}$ Schilken, ZZP 109 (1996), S. 315, 322

${ }^{821}$ Storme, Rapprochement, S. 147; Schilken, ZZP 109 (1996), S. 315, 320

${ }^{822}$ Das legt die französische Fassung des Art 11.4 (le juge) nahe.

${ }^{823}$ Dafür spricht die englische Fassung (the court).

${ }^{824}$ Bei Zahlung innerhalb der 8-Tages-Frist soll der Schuldner von einer Kostenfolge verschont bleiben. Gerichtskosten fallen so lange nicht an. Der Gläubiger muss die Kosten seiner Rechtsverfolgung nach allgemeinen zivilrechtlichen Regeln geltend machen. Schilken, ZZP 109 (1996), S. 315, 323, Fn. 34
} 
ten Formular einlegen, Art. 11.6. Legt der Schuldner fristgerecht Widerspruch ein, wird das Verfahren im normalen Rechtsgang weitergeführt, Art. 11.7. Wird innerhalb der zwei Wochen nach Zustellung kein Widerspruch eingelegt, erwächst der Mahnbescheid in einen formell und materiell rechtskräftigen Zahlungstitel, Art. 11.8. Die Verjährung wird mit Zustellung des Mahnbescheides unterbrochen.

\section{Rechtsvergleichende Betrachtung}

Der Entwurf folgt in seiner angestrebten Verfahrensweise dem deutschen und englischen Einleitungskonzept, wonach das einleitende Dokument erst bei Gericht eingereicht und sodann dem Beklagten zugestellt wird. Ob die Zustellung vom Gericht vorgenommen wird, wie in Deutschland und neuerdings im Regelfall auch in England, oder ob dies der Kläger tut, lässt der Entwurf offen. Die gemeinsame Verfahrenseinleitung durch beide Parteien ist sowohl dem englischen als auch dem deutschen Recht fremd und steht unter dem Vorbehalt der nationalen Regelung. ${ }^{825}$

Inhaltlich fehlen im Entwurf diverse Vorschriften. In subjektiver Hinsicht fehlt eine Vorschrift, die bestimmt, wie genau Parteien und Gericht bezeichnet werden müssen. Den Normen über das Mahnverfahren lässt sich dazu zwar entnehmen, dass diese Bezeichnung vorgenommen werden muss, aber Angaben zur Genauigkeit finden sich nicht. Die ausführliche Regelung zu Namen und Adresse des Anwalts steht dazu in einem auffälligen Missverhältnis; insbesondere, weil es sich dabei um eine Vorschrift handelt, die den reibungslosen Verfahrensablauf garantiert, aber als solche nur Ordnungscharakter hat. Die materiellrechtlich und auch prozessual wesentlich wichtigere Regel zur Parteinämlichkeit fehlt dagegen. Hier sind das deutsche und das englische Recht, die die Angaben zum Prozessvertreter als Zweckmäßigkeitsvorschriften begreifen, dem Entwurf überlegen.

Ein Unterschriftserfordernis nennt der Entwurf nicht. Ob und wodurch die Identifikations- und Verifikationsfunktion gewahrt werden soll, ist unklar. Hier wäre zumindest das Unterschriftserfordernis angebracht gewesen, wenn nicht sogar eine Wahrheitsversicherung wie nach den CPR 1998.

In objektiver Hinsicht entspricht der Entwurf weitgehend dem deutschen und neuen englischen Recht. Antrag und Tatsachen zur Identifikation des Streitgegenstandes müssen genannt werden. Wann ein substantiierter Vortrag spätestens folgen muss, läßt sich dem Entwurf nicht entnehmen. Die sehr schwammige Praktikabilitätsklausel scheint wenig hilfreich. Beweismittel sollen schon in der Klage genannt werden, allerdings unter dem Vorbehalt der Praktikabilität. An dieser Stelle besteht Parallelität zum deutschen Recht, das über $\S 130$ Nr. 5 ZPO eine entsprechende Sollvorschrift kennt. Das englische Recht hat sich diesem Prinzip zumindest formal angenähert; dabei ist jedoch $\mathrm{zu}$ beachten, dass der englische Kläger Beweismittel anführen darf, davon aber zumindest noch abgeraten wird. ${ }^{826}$ Das Anführen von Rechtsansichten ist sowohl nach deutschem und englischem Recht, als auch nach dem Entwurf erlaubt, aber nicht vorgeschrieben.

Nach allen drei Systemen ist der Beklagte zusammen mit der Klage über seine Handlungsmöglichkeiten und die möglichen Folgen zu informieren.

Die Pflicht, einen Zustellungsbevollmächtigten im Inland anzugeben, entspricht $\S 174$ II ZPO a.F. recht genau und funktional $\S 184$ I 1 ZPO n.F. und dient der Vereinfachung der Kommunikation zwischen allen Verfahrensbeteiligten. Im

\footnotetext{
${ }^{825}$ Es handelt sich dabei um ein französisches Rechtsinstitut, die requête conjointe. Schelo, S. 121

${ }^{826}$ Commercial Court Guide, Section C 1.2 i
} 
englischen Recht findet sich r. 6.5 (2) CPR 1998 als Pendant dazu. Danach muss eine Partei eine inländische Zustellungsadresse angeben.

Bezüglich der Zustellung beläßt es der Entwurf weitgehend bei den nationalen Regeln, betont aber ausdrücklich die Subsidiarität der Ersatzzustellung. Das entspricht der gesetzlichen Regel in Deutschland und England. Die Ersatzzustellung ist nur vorzunehmen, wenn die persönliche Zustellung misslingt. Vom gesetzgeberischen Regelungsziel her, rennt der Entwurf damit offene Türen ein. Die Zustellungspraxis sieht freilich anders aus. ${ }^{827}$

Bei den Wirkungen der Klageerhebung sieht der Entwurf vor, dass die Klage mit Einreichung bei Gericht rechtshängig wird und die Rechtshängigkeit rückwirkend entfällt, wenn die Klageschrift nicht binnen drei Monaten zugestellt wird. Insoweit besteht ein Unterschied zum deutschen und zum englischen Recht. Nach $\S 167$ ZPO n.F. treten die Wirkungen der Rechtshängigkeit (die erst mit Zustellung existiert) schon bei Einreichung des Schriftsatzes bei Gericht ein, wenn die Klage demnächst zugestellt wird. In England herrschen unterschiedliche Regimes. Für das lis alibi pendens kommt es national auf die Ausstellung der Claim form und international auf deren Zustellung an. Die Unterbrechung der Verjährung erfolgt einheitlich bei Ausstellung der Claim form. Die Lösung des Entwurfs hat den Vorteil der Einfachheit, Klarheit und Rechtssicherheit für sich. Er leidet jedoch an der Starrheit der Drei-Monats-Frist. Obliegt es nämlich dem Gericht, die Klage zuzustellen und muss diese in ferne ggf. abgelegene ländliche Gebiete eines Landes mit schlecht ausgeprägter Infrastruktur zugestellt werden, verstreicht die Frist unter Umständen ohne ein Verschulden des Klägers. Hier wäre eine dynamische Lösung oder ein Auffangtatbestand angebracht.

Die Einleitung im Mahnverfahren hat der deutschen Regelung gegenüber Vorund Nachteile. Als vorteilhaft wird man die Schlüssigkeitsprüfung bezeichnen müssen, auch wenn dies das Verfahren verlangsamt und den Arbeitsaufwand erhöht. Prozessökonomie ist kein Selbstzweck und die Häufung von Klagen gegen sittenwidrig erschlichene Vollstreckungsbescheide in Deutschland ist ein klares Anzeichen dafür, dass das Verfahren hierzulande zum Teil missbraucht wird. ${ }^{828}$ Dem würde eine Schlüssigkeitsprüfung entgegenwirken. Abzulehnen ist hingegen die Ausgestaltung des Mahnverfahrens als Urkunden-Mahnverfahren. Der Vorteil für die materielle Gerechtigkeit, der durch eine kleine Beweisaufnahme gegebenenfalls erzielt wird, steht in keinem Verhältnis zu den Nachteilen. In der großen Mehrzahl der Streitigkeiten sind keine Urkunden vorhanden, die den Anspruch hinreichend belegen. In diesen Fällen wäre der Weg über das Mahnverfahren auch bei unbestrittenen Ansprüchen versperrt. Selbst wenn Urkunden vorhanden sind, bleibt immer noch fraglich, ob diese dem Gericht ausreichen, z.B. wenn es sich nur um Indizurkunden handelt. Dann läuft der Kläger Gefahr, Zeit und Geld in ein Verfahren zu investieren, von dem er nicht abschätzen kann, ob es erfolgversprechend ist.

Sieht man von der aus der Schlüssigkeitsprüfung folgenden Pflicht zur Anspruchsbegründung und dem Urkundenerfordernis ab, entspricht das Mahnverfahren seinem deutschen Pendant mit Ausnahme der Tatsache, dass eine Einspruchsmöglichkeit nach Versäumung des Widerspruchs nicht möglich ist. Den Beklagtenschutz, den die Schlüssigkeitsprüfung verspricht, hebt die Beschneidung der Widerspruchsmöglichkeiten wieder etwas auf.

\footnotetext{
${ }^{827}$ Roth, ZZP 109 (1996), S. 271, 285

${ }^{828}$ Nach Schilken sei die Gewährung des materiellen Rechts durch die Abschaffung der Schlüssigkeitsprüfung im deutschen Mahnverfahren geradezu konterkariert worden. Lemken, ZZP 109 (1996), S. 343
} 
Insgesamt macht der Entwurf einen eher unausgewogenen Eindruck. Insbesondere die zahlreichen Lücken machen eine Beurteilung schwierig. Aus rechtsvergleichender Sicht enthält er nur wenige Alternativen, die dem deutschen und englischen Funktionspendants überlegen scheinen. $\mathrm{Zu}$ nennen ist hier die Vorverlegung der Rechtshängigkeit auf die Einreichung des einleitenden Schriftstücks unter rückwirkendem Wegfall, wenn die Zustellung nicht innerhalb von drei Monaten erfolgt. Allerdings fehlt insoweit eine Auffangnorm, die eventuelle Härten für den Kläger abfedert. Eine einheitliche Regelung täte insbesondere dem englischen Recht gut, das sich insoweit eher inkonsistent darstellt. Begrüßenswert ist auch die (Wieder-) Einführung der Schlüssigkeitsprüfung im Mahnverfahren. Beschränkt man sich jedoch auf das einleitende Schriftstück selbst, zeichnet sich der Entwurf hauptsächlich durch Lücken und Ungenauigkeiten aus. Die Prioritätensetzung ist zum Teil schwer nachzuvollziehen. Die ,perfektionistische“ ${ }^{629}$ Vorschrift zu Namen und Adresse des Anwalts steht in keinem Verhältnis zu den Vorschriften über die Parteien. Auch das Praktikabilitätskonzept ist schwer zu fassen. Es wäre eher von Vorteil, zwingende Anforderungen an die Essentialia einer Klageschrift zu stellen und weitere zweckmäßige Angaben als Sollvorschriften zu formulieren, wie dies die ZPO und die CPR 1998 inklusive Practice Directions handhaben.

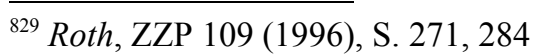




\section{Principles and Rules of Transnational Civil Procedure}

Einen anderen Ansatz verfolgt das American Law Institute (ALI): eine Zivilprozessordnung für transnationale Prozesse, die die regelmäßig dabei auftretenden Probleme regelt und damit für prozessuale Rechtssicherheit und Gerechtigkeit sorgt.

Das ALI erstellt in Zusammenarbeit mit Unidroit Diskussionsentwürfe für sog. „Principles and Rules of Transnational Civil Procedure“6830. Diese beschränken sich auf internationale geschäftliche Transaktionen.

Die Principles and Rules of Transnational Civil Procedure sind der Entwurf zu einem Modellgesetz für transnationale Prozesse aufgrund geschäftlicher Transaktionen, die das American Law Institute seit 1999 in Zusammenarbeit mit Unidroit $^{831}$ erstellt. Vorliegend wird die dritte Fassung des Entwurfs (Stand April 2002) betrachtet.

Der Entwurf ist zweigeteilt in die zugrundeliegenden Prinzipien (Principles) und die detaillierten Regeln (Rules). Dabei enthalten die Prinzipien die Interpretationsgrundlagen für die Regeln. ${ }^{832}$

\section{Anwendungsbereich}

Nach r. 2.1 finden die Regeln auf Streitigkeiten aufgrund transnationaler geschäftlicher Transaktionen Anwendung. Darunter versteht der Entwurf vertragliche, wie dingliche Klagen, Streitigkeiten über gewerblichen Rechtsschutz und deliktische Ansprüche, soweit es sich um „geschäftliche Delikte“ handelt. ${ }^{833}$ Ausdrücklich ausgenommen werden Klagen wegen Körperverletzung (personal injury) und rechtswidriger Tötung (wrongful death), weil es sich dabei um Bereiche handelt, bei denen in den USA Geschworenengerichte als unersetzlich erachtet werden. Daher halten die Autoren eine internationale Vereinheitlichung für ausgeschlossen. ${ }^{834}$

Das Verfahren soll grundsätzlich aus drei Teilen bestehen, p. 8.1 PoTCP:

Eine „Pleading“-Phase, beginnend mit der Klageerhebung, in der durch Schriftsatzaustausch die streitigen Tatsachen soweit konkretisiert werden, dass eine Beweisaufnahme möglich wird, p. 8.2 PoTCP.

Eine Zwischenphase (Interim Phase), bestehend aus einem ersten Verhandlungstermin, in dem der Verfahrensgang festgelegt wird. Des weiteren klärt das Gericht in dieser Phase Fragen zur Zulässigkeit oder andere Punkte, die das Verfahren frühzeitig entscheiden könnten, beschäftigt sich mit der Verfügbarkeit von Beweismitteln und ordnet gegebenenfalls die Erhebung der Beweise an.

In der Hauptverhandlung (Final Hearing) findet die noch ausstehende Beweisaufnahme statt und die Parteien können ihre Argumente mündlich vortragen.

\section{Verfahrenseinleitung}

Die Verfahrenseinleitung erfolgt nur auf Parteiinitiative hin, p. 9.1 PoTCP, und der Verfahrensgegenstand wird durch den Parteivortrag bestimmt, p. 9.2 PoTCP. Demnach hält sich der Entwurf strikt an Dispositionsmaxime und Verhandlungsprinzip. Die Klageeinleitung erfolgt nur über das „normale“ System mit Klage-

\footnotetext{
${ }^{830}$ Der erste Entwurf hieß noch „Transnational Rules of Civil Procedure“

${ }^{831}$ Die Zusammenarbeit mit Unidroit besteht erst seit 2000.

${ }^{832}$ ALI/Unidroit, S. 5

${ }^{833}$ ALI/Unidroit, S. 56

${ }^{834}$ ALI/Unidroit, Preface, S. XX
} 
schrift. Eine Einleitung mit Hilfe eines Mahnverfahrens oder eines vergleichbaren Institutes sieht der Entwurf nicht vor.

\section{a. Allgemeines Verfahren}

Das Verfahren wird durch Einreichen der Klageschrift bei Gericht begonnen, r. 11.1 RoTCP. Daraufhin wird die Klage dem Beklagten zugestellt. Wer die Zustellung vornehmen muss, legt der Entwurf nicht fest. Die Kommentierung scheint eine Zustellung durch das Gericht nahezulegen, mit letzter Gewissheit läßt sich dies jedoch nicht sagen. Der Zeitpunkt der Einreichung bei Gericht entscheidet über die Verjährungsunterbrechung und die entgegenstehende Rechtshängigkeit, r. 11.2 RoTCP. Die Tatsache, dass es keine Norm gibt, die bestimmt, innerhalb welcher Zeit zugestellt werden muss, stützt auch die Vermutung, dass die Zustellung dem Gericht obliegt, nicht dem Kläger. Andernfalls könnte der Kläger die Verjährungsunterbrechung bewirken, ohne tatsächlich ein Verfahren anzustrengen, indem er dem Beklagten die Klage einfach nicht zustellt. Wie die Gerichte bei entgegenstehender Rechtshängigkeit entscheiden sollen, legt der Entwurf nicht fest. ${ }^{835}$

Das Gericht ist befugt, die Parteien oder Dritte mit Sanktionen zu belegen, wenn sie den Anordnungen des Gerichts nicht Folge leisten, p. 11 PoTCP. Dabei scheint die Art der Sanktionen im Ermessen des Gerichts zu liegen. P. 11.2 PoTCP legt nur fest, dass die Sanktionen vernünftig und angemessen sein sollen. Welche Rechtsfolgen es hat, wenn das Gericht Fehler macht, z.B. eine Zustellung nicht vornimmt, legt der Entwurf nicht fest.

\section{b. Inhalt der Klageschrift}

Die Klageschrift ist in r. 12 RoTCP geregelt. Es wird nicht zwischen einem Ladungsbefehl (Writ, Claim form, Summons) bzw. Klageerhebung und der Klagebegründung unterschieden. Statt dessen geht der Entwurf von einer einheitlichen Klageschrift (Statement of Claim (Complaint)) als Einleitungsdokument mit Begründung aus. ${ }^{836}$

Die Vorschriften regeln die objektiven Inhalte der Klageschrift. Sowohl in subjektiver als auch in formaler Hinsicht fehlt aber jegliche Regelung, d.h. der Entwurf schweigt zu Namen und Anschriften der Parteien, Bezeichnungen ihrer Vertreter, der Angabe des Gerichts und einem eventuellen Unterschriftserfordernis oder einer Versicherung der Wahrheit. Das verwundert insbesondere, da es sich dabei um Kriterien handelt, die im Common Law und im Civil Law weitgehend gleich gehandhabt werden und zu den unverzichtbaren Essentialia der Klageschrift gehören. Insoweit gilt dann die jewilige lex fori.

In objektiver Hinsicht muss der Kläger seinen Antrag angeben. Der Entwurf spricht insoweit vom begehrten Urteilsausspruch (judgement requested), r. 12.5 RoTCP. Dabei muss er eine Zahlungsklage beziffern und andere gewünschte Aussprüche genau angeben. Das Gericht ist durch die Höhe des Antrags nach oben gebunden. ${ }^{837}$ Dies wird erstaunlicherweise nicht mit der Dispositionsmaxime, sondern mit dem Beklagtenschutz begründet. Er soll sich im Falle der Säumnis auf die maximale Urteilshöhe verlassen können. ${ }^{838}$

\footnotetext{
${ }^{835}$ p. 251 PoTCP bestimmt lediglich, dass bei der Entscheidung über die entgegenstehende Rechtshängigkeit der Streitgegenstand durch die Schriftsätze der Parteien bestimmt wird.

${ }^{836}$ Zustimmend, allerdings zum ersten Entwurf, äußert sich Stürner, ZZP 112 (1999), S. 185,195.

${ }^{837}$ ALI/Unidroit, S. 71

${ }^{838}$ ALI/Unidroit, S. 71
} 
Ferner muss der Kläger die Tatsachen, auf die er seine Klage stützt, und die dazugehörigen Beweismittel angeben, r. 12.1 RoTCP. Sofern es vernünftigerweise praktikabel (reasonably practicable) ist, muss er in tatsächlicher Hinsicht substantiiert vortragen. ${ }^{839}$ Bei der Beurteilung, ob der Vortrag ausreichend substantiiert ist, soll das Gericht in Betracht ziehen, dass sich Fakten oder Beweismittel ggf. erst im Verfahren ergeben können, r. 12.3 Satz 2 RoTCP. Der Vortrag des Klägers muss schlüssig sein. Dabei handelt es sich zwar nicht um eine Voraussetzung für die Einleitung des Verfahrens, aber ein Versäumnisurteil kann nur bei schlüssiger, mit Beweisantritt versehener Klage ergehen, r. 15.2.3 RoTCP.

Hat sich der Kläger zu einem Schieds-, Schlichtungs- oder vergleichbaren Verfahren verpflichtet, muss er dies in der Klage angeben, r. 12.4 RoTCP.

Schließlich muss der Kläger in der Klage die Rechtsgründe seines Anspruchs inklusive des ausländischen und internationalen Privatrechts angeben sowie die Normen, aus denen sich die Anwendbarkeit der Principles and Rules of Civil Procedure ergibt, r. 12.1 RoTCP. Dies muss ausreichend sein, damit das Gericht deren Gültigkeit für den konkreten Fall prüfen kann, r. 12.2 RoTCP. Trotz dieser strengen Regel zur Angabe von Rechtsausführungen gilt der Iura-Novit-CuriaGrundsatz. ${ }^{840}$ Der gewählte Regelungsweg ergibt sich dabei als Kompromiss aus verschiedenen Systemen. Zum einen ist es in diversen Rechtsordnungen vorgeschrieben, Rechtsausführungen zu machen, zum anderen handelt es sich bei transnationalen Verfahren in der Regel um Prozesse, in denen internationales Privatrecht und/oder ausländisches Recht zur Anwendung kommt. Bei der Anwendung ausländischen Rechts sind die Systeme wiederum zweigeteilt. Entweder es wird als Tatsache behandelt, die die Parteien beweisen müssen. Gelingt das nicht, wird nationales Sachrecht angewandt. Oder es handelt sich bei ausländischem Recht prozessual auch um Recht im engeren Sinne und der Iura-Novit-Curia-Grundsatz gilt auch insoweit. Das letztere System gilt beispielsweise in Deutschland mit der Einschränkung, dass die Parteien dazu Beweis antreten können, § 293 ZPO. Der Entwurf wählt einen Mittelweg. Zwar müssen die Parteien zur Unterstützung des Gerichts Rechtsausführungen machen, letztlich entscheidet aber das Gericht über die rechtlichen Grundlagen seiner Entscheidung, p. 19.1 PoTCP.

\section{c. Zustellung}

$\mathrm{Zu}$ den Arten und dem Verfahren der Zustellung schweigt der Entwurf. Es ist dabei aber nicht ganz klar, ob die Zustellung grundsätzlich nationalem Recht überlassen werden soll. Der erste Entwurf verwies insofern noch auf anwendbare internationale Konventionen. ${ }^{841} \mathrm{Im}$ seinem Anwendungsbereich regelt das HZÜ die Zustellung. R. 7 RoTCP legt nur fest, dass der Beklagte über die Klage formell benachrichtigt werden muss. Dazu können Mittel gewählt werden, die vernünftigerweise für effektiv gehalten werden dürfen. Daraus lässt sich schließen, dass das Gericht in freiem Ermessen über die Art der Zustellung entscheiden soll, r. 7.1 RoTCP. Welcher Mittel es sich dabei bedienen darf, bleibt unklar.

Die zugestellte Benachrichtigung muss in der Sprache des Gerichts verfasst sein und in der Sprache des Beklagten, bzw. der Sprache der Geschäftsbeziehung, aus der der Streit entstanden ist, r. 7.3 RoTCP. Inhaltlich muss die Benachrichtigung eine Kopie der Klageschrift enthalten, r. 7.2.1 RoTCP, angeben, dass für das

\footnotetext{
${ }^{839}$ r. 12.3 Satz 1 RoTCP: „,The statement of facts must, so far as reasonably praticable, set forth detail as to time,place, participants, and events."

${ }^{840}$ ALI/Unidroit, S. 71

${ }^{841}$ Vgl. Walter, ZZP 112 (1999), S. 204, 206; Stürner, ZZP 112 (1999), S 185, 195
} 
Verfahren die Transnational Rules of Civil Procedure anwendbar sind, r. 7.2.2 RoTCP, die Frist zur Klageerwiderung festsetzen und die Folgen der Säumnis angeben, r.7.2.3 RoTCP.

Die Regelfrist zur Klageerwiderung soll 60 Tage betragen, wobei das Gericht frei ist, eine längere Frist zu bestimmen, r. 13.1 RoTCP.

\section{Rechtsvergleichende Betrachtung}

Die Principles and Rules of Transnational Civil Procedure versuchen einen Spagat zwischen Civil Law und Common Law amerikanischer Prägung. Im Bewusstsein der diversen unüberbrückbaren Differenzen beschränkt sich der Entwurf auch nur auf geschäftliche Transaktionen. Obwohl ein Kompromiss dadurch erst möglich wurde, wird der Entwurf in seinem Anwendungsbereich damit erheblich beschnitten. Dies gilt insbesondere, da im internationalen Geschäftsverkehr Schiedsvereinbarungen üblich sind und die Principles and Rules of Transnational Civil Procedure damit wohl eher selten zur Anwendung kämen.

Nichtsdestotrotz bieten sie einige interessante Ansatzpunkte bei der Verfahrenseinleitung an.

Wie im deutschen und im englischen Recht wird das Verfahren durch die Einreichung des einleitenden Schriftstücks bei Gericht begonnen.

Wem die Zustellung der Klage an den Beklagten obliegt, bleibt offen, es liegt jedoch nahe, dass dies Aufgabe des Gerichts ist. Auch insoweit entspricht der Entwurf dem deutschen und englischen System. Eine sehr klare Linie findet der Entwurf bei der Frage, wann die Klage rechtshängig wird. Dies geschieht mit Klageeinreichung bei Gericht. Daran anschließend treten auch die Rechtsfolgen der Rechtshängigkeit (lis alibi pendens, Verjährungsunterbrechung) ein. Obwohl die Regel auf den ersten Blick durch ihre Einfachheit besticht, fehlt eine Vorschrift, die dafür sorgt, dass die Zustellung innerhalb einer bestimmten Zeit erfolgen muss, damit die Wirkungen Bestand haben. Der Entwurf geht scheinbar davon aus, dass das Gericht schon für eine zeitnahe Benachrichtigung sorgen wird. Ist dies jedoch nicht der Fall, bleibt der Beklagte ungeschützt. Welche Rechtsfolgen in diesem Fall eintreten, bleibt offen.

$\mathrm{Zu}$ den subjektiven und formellen Anforderungen an das einleitende Schriftstück schweigt der Entwurf genauso wie der Storme-Entwurf. Dies ist unverständlich, wie schon zum Storme-Entwurf ausgeführt wurde. ${ }^{842}$

Anders als in England (und rechtstheoretisch auch in Deutschland) wird beim einleitenden Schriftstück nicht zwischen Ladungsbefehl (Writ, Claim form) und Begründung (Particulars of claim) unterschieden. Der Kläger muss in der Klage neben seinem Antrag auch die Tatsachen substantiiert vortragen, auf die er seinen Anspruch stützt. Darin unterscheidet sich der Entwurf vom deutschen und englischen Recht. In England muss die Begründung nicht mit der Claim form zugestellt werden, diese muss nur den Streitgegenstand individualisieren. Die deutsche Rechtslage stellt sich grundsätzlich genauso dar. In der Klage selbst muss nur der Streitgegenstand festgelegt werden, die Begründung muss spätestens nach Aufforderung durch das Gericht folgen. In der Praxis ist der Unterschied freilich eher zu vernachlässigen. In Deutschland wird die Klagebegründung üblicherweise mit der Klageschrift eingereicht, in England muss sie der Claim form spätestens nach 14 Tagen nachfolgen. Trotz des geringen praktischen Unterschiedes ist die Fassung des Entwurfs zu begrüßen. Es ist verfahrensökonomischer und

${ }^{842}$ S.o. D.I.2 
dem Kläger auch ohne weiteres zumutbar, dass er seine Klage bei Einreichung, so weit wie möglich substantiiert, begründet.

Bezüglich des Antrags entspricht der Entwurf dem deutschen Recht. Zahlungsanträge sind zu beziffern, das Gericht ist an den Antrag nach oben gebunden. Auch diese Regelung entspricht deutschem Recht und unterscheidet sich insoweit von den CPR 1998. Dies ist aus zwei Gründen auch angebracht. Zum einen soll das Gericht nach r. 18.3.1 RoTCP auf das Stellen der richtigen Anträge und die gegebenenfalls erforderliche Klageerweiterung hinweisen, wie dies auch §139 ZPO regelt. Zum anderen handelt es sich bei den PRoTCP um Regeln für Prozesse aufgrund geschäftlicher Transaktionen. Die englische Regel, wonach das Gericht auch über den Antrag hinausgehen darf, hat den Sinn, anwaltlich nicht vertretene oder anderweitig schutzwürdige Kläger angemessen behandeln zu können. Solche Konstellationen liegen bei transnationalen kommerziellen Disputen in der Regel nicht vor.

Aus prozessökonomischer Sicht ist es sinnvoll, Beweismittel so früh wie möglich anzuführen, wie dies der Entwurf vorsieht. Auch insoweit besteht eine Parallele zum deutschen Verfahren. § 130 Nr. 5 ZPO ist zwar nur Sollvorschrift, in der Praxis werden aber Beweismittel meist frühzeitig benannt. Den gleichen Effekt erzielt im Entwurf der Praktikabilitätsvorbehalt der r. 12.3 RoTCP. Grundsätzlich sind die Beweismittel in der Klage zu benennen, aber eben nur soweit es praktikabel ist und vernünftigerweise erwartet werden kann. Hier ergibt sich ein erheblicher Unterschied zum englischen Recht. Dort ist zwar die Angabe von Beweismitteln in den particulars zwischenzeitlich erlaubt, aber weder vorgeschrieben noch der praktische Alltag.

Eine interessante Abweichung vom englischen und deutschen Recht existiert weiterhin bei der Angabe von Rechtsausführungen. Diese sind in England und Deutschland erlaubt, der Entwurf hingegen sieht sie zwingend vor, obwohl auch nach den PRoTCP der Grundsatz Iura-Novit-Curia gilt. Der Unterschied zur deutschen und englischen Regelung soll sich aus der Tatsache ergeben, dass in transnationalen Verfahren regelmäßig internationales Privatrecht und oft ausländisches Recht Anwendung findet. Ob Klippen, die insoweit entstehen, durch Rechtsausführungen der Parteien umschifft werden, ist zweifelhaft. Der Entwurf stellt einen Kompromiss dar, der zwar ausländisches Recht nicht als beweisbare Tatsache ansieht, die Parteien aber dennoch zum Rechtsvortrag zwingt. Der Ansatz, ausländisches Recht als Recht zu behandeln, ist in transnationalen Verfahren sicher richtig. Andernfalls würde die lex fori als Auffangrechtsordnung zu viel Bedeutung gewinnen und dadurch würde das forum shopping, dem der Entwurf auf prozessualer Ebene entgegenwirken will, auf der Ebene des Sachrechts wieder attraktiv. Die Pflicht zu Rechtsausführungen steht aber in konzeptionellem Widerspruch zur Stellung des Gerichts im Entwurf. Da das Gericht auch ausländisches Recht und insbesondere das eigene Kollisionsrecht kennen muss, stellen die Rechtsausführungen der Parteien eher Makulatur dar. Nicht zuletzt, weil der Entwurf auch keine konkreten Sanktionen vorsieht, wenn eine Partei keine oder unzulängliche Ausführungen macht. Insoweit wählen das englische und deutsche Recht eine stringentere Lösung.

Die Vorschriften zur Benachrichtigung entsprechen weitgehend den anderen dargestellten Rechtsordnungen. Mit der Klage ist eine Frist zur Klageerwiderung und die Belehrung über mögliche Folgen der Säumnis zuzustellen. Aus dem Anwendungsbereich der PRoTCP ausschließlich für transnationale kommerzielle Streitigkeiten ergibt sich der Ausnahmecharakter eines Verfahrens nach dem 
Entwurf, so dass es sinnvoll ist, dem Beklagten auch mitzuteilen, dass die PRoTCP Anwendung finden. Auch die Mindestfrist von 60 Tagen zur Klageerwiderung scheint aufgrund der Schwerfälligkeit des internationalen Rechtsverkehrs angebracht.

Zur Zustellung schweigt der Entwurf mit Ausnahme von r. 7 RoTCP, wonach das Gericht bei der Benachrichtigung des Beklagten von der Verfahrenseröffnung alle Mittel wählen kann, die ihm vernünftig und effektiv erscheinen. Einen Vorrang der persönlichen Zustellung normiert der Entwurf demnach nicht.

Die Principles and Rules of Transnational Civil Procedure sind als Diskussionsentwurf in der dritten Generation schon wesentlich ausgewogener als der StormeEntwurf. Dabei profitieren sie auch von ihrer Konzeption als Prozessrecht, das ausschließlich bei transnationalen kommerziellen Streitigkeiten Anwendung findet. Der eingeschränkte Anwendungsbereich stellt aber gleichzeitig einen großen Nachteil des Entwurfs dar - nicht zuletzt, da das Kriterium der ,geschäftlichen Streitigkeit“ zu Abgrenzungsschwierigkeiten führen wird.

Das Prinzip der Verfahrenseinleitung ist klar und einfach definiert. Einige Bereiche sind jedoch, bewusst oder unbewusst, ausgelassen worden. Hier besteht teils noch erheblicher Nachholbedarf. Dies gilt insbesondere für die Frage, wer innerhalb welchen Zeitraums dem Beklagten die Klage zustellt.

Inhaltlich fehlen leider noch alle subjektiven (Parteien, Gericht) und formalen Anforderungen (Unterschrift, Wahrheitsversicherung) an die Klageschrift. Objektiv bietet der Entwurf einige interessante Ansätze, die einer modernen Zivilprozessordnung gut zu Gesicht stehen. Hier ist besonders das einheitliche Einleitungsdokument zu nennen, in dem die Klage soweit möglich schon substantiiert und unter Beweisangebot begründet wird. Als verfehlt wird man hingegen die Vorschriften $\mathrm{zu}$ den Rechtsausführungen bezeichnen müssen. Es ist zwar zu begrüßen, dass ausländisches Recht nicht als Tatsache betrachtet wird. Wenn die rechtliche Beurteilung aber allein dem Gericht obliegt, wie p. 19.1 PoTCP festschreibt, ist es wenig sinnvoll, den Parteien Rechtsausführungen vorzuschreiben. Wird nach ausländischem Recht verhandelt, werden sie es ohnehin tun, um das Gericht von ihrer Rechtsansicht zu überzeugen. Gleiches gilt für ungewöhnliche oder sehr spezielle Rechtsgebiete. In allen anderen Bereichen muss das Gericht ohnehin ausreichende Kompetenz zur rechtlichen Beurteilung des Sachverhaltes haben. Durchführbar ist die Pflicht zur Rechtsausführung ohnehin nur, wenn man gleichzeitig die Anwaltspflicht vorschreibt, wovon der Entwurf stillschweigend auszugehen scheint. ${ }^{843}$

Im Zustellungsrecht wäre eine eindeutige Regelung zu wünschen, entweder zugunsten nationalen Rechts bzw. internationaler Übereinkommen oder einer eigenen Regelung. Entscheidet man sich zugunsten der ersten Variante, sollte der Entwurf rechtliche Mindeststandards für die Zustellung fixieren.

${ }^{843}$ ALI/Unidroit, S. 65 


\section{EuGVÜ/EuGV-VO}

Einen dritten Weg stellen. Konventionen dar, die die Zuständigkeit, Zustellung sowie Anerkennung und Vollstreckung ausländischer Urteile regeln.

Dabei bleibt es bei den nationalen Zivilprozessordnungen. Diese werden nur in internationalen Fällen, wie immer diese auch definiert werden, von den einschlägigen Konventionen modifiziert. Bekannteste und erfolgreichste Beispiele hierfür sind das EuGVÜ, sowie sein Nachfolger EuGV-VO, LugÜ, und HZustÜ. Ein dem EuGVÜ weitgehend entsprechendes Projekt treibt die Haager Konferenz für internationales Privatrecht voran.

Hier soll das EGVÜ/ die EuGV-VO betrachtet werden.

\section{Verfahrenseinleitung in EuGVÜ/EuGV-VO}

Die Verfahrenseinleitung berührt das EuGVÜ/die EuGV-VO nur an zwei Stellen: Zum einen bei der Rechtshängigkeit und im Zusammenhang stehenden Verfahren (lis alibi pendens), ${ }^{844}$ zum anderen bei der Anerkennung ausländischer Urteile, da das verfahrenseinleitende Schriftstück dem Beklagten „ordnungsgemäß und [...] so rechtzeitig“ ${ }^{645}$ bzw. „so rechtzeitig und in einer Art und Weise ${ }^{\text {c846 }}$ zugestellt worden sein muss, dass er sich verteidigen kann. ${ }^{847}$

Obwohl beide Vorschriften die Verfahrenseinleitung nur streifen, sind sie von großer instruktiver Bedeutung für die Diskussion um eine einheitliche Verfahrenseinleitung.

a. Das verfahrenseinleitende Schriftstück, Art. 27 Nr.2 EuGVÜ und Art. 34 Nr. 2 EuGV-VO

Nach Art. 27 Nr. 2 EuGVÜ; Art. 34 Nr. 2 EuGV-VO muss dem Beklagten das einleitende Schriftstück zugestellt worden sein. Eine Definition des einleitenden Schriftstücks findet sich nur insoweit in der EuGH-Diktion, als dass es sich um das Schriftstück handelt, dessen rechtzeitige Zustellung den Beklagten in die Lage versetzt, seine Rechte vor Erlass einer vollstreckbaren Entscheidung im Urteilsstaat geltend zu machen. ${ }^{848}$ Eine genaue Definition ist auch nicht gewollt, bzw. nur insoweit, als sie dem Ziel der Vorschrift genügt. Die ratio legis ist der Schutz des Beklagten vor Urteilen, die in einem Verfahren ergangen sind, in dem er sich nicht verteidigen konnte. ${ }^{849}$ Daher muss das Schriftstück ein Verfahren einleiten, das zu einem Titel führen kann, der im Rahmen des EuGVÜ/der EuGV-VO anerkennungs- und vollstreckungsfähig ist ${ }^{850}$ und es muss den Beklagten über „die Elemente des Rechtsstreits in Kenntnis ${ }^{\text {(651 }}$ setzen. $^{852}$

\footnotetext{
${ }^{844}$ Art. 21 ff. EuGVÜ; 27 ff. EuGV-VO

${ }^{845}$ Art. 27 Nr. 2 EuGVÜ

${ }^{846} 34$ Nr. 2 EuGV-VO

${ }^{847}$ „Ordnungsgemäß“ wurde in der EuGV-VO gestrichen, so dass es nun nicht mehr darauf ankommt, ob die Zustellung ordnungsgemäß erfolgt ist, sondern ob sich der Beklagte in Folge des Zeitpunkts oder der Art und Weise der Zustellung nicht verteidigen konnte. Kropholler, Art. 34 Rn. 33

${ }^{848}$ EuGH Hengst Import/Campese Slg. 1993 I, S. 2113 Nr. 19

${ }^{849}$ Frank, S. 212

${ }^{850}$ EuGH Klomps/Michel Slg. 1981, S. 1593

${ }^{851}$ EuGH Sonntag/Waidmann Slg. 1993 I, S. 1963 Nr. 39

${ }^{852}$ Frank, S. 213
} 
Damit erfasst das EuGVÜ/die EuGV-VO alle Schriftstücke, die Verfahren nach nationalem Recht einleiten können, insbesondere auch deutsche Mahnbescheide. ${ }^{853}$

Inhaltlich muss das Schriftstück einen Antrag enthalten, dem der Beklagte entnehmen kann, was für eine Verurteilung ihm droht. Bei unbezifferten Anträgen ist es demnach erforderlich, dass der Beklagte die Größenordnung abschätzen kann. ${ }^{854}$ Des Weiteren muss das einleitende Schriftstück auch den Lebenssachverhalt anführen, auf den sich der Anspruch stützt. Andernfalls könnte der Beklagte den Rechtsstreit nicht identifizieren.

Bei diesen Kriterien handelt es sich um die Bestimmung des Streitgegenstandes, der aufgrund des Regelungszweckes des EuGVÜ/der EuGV-VO autonom zu bestimmen ist. Dies entspricht auch weitgehend der Definition des $\mathrm{EuGH}^{855}$ zum Streitgegenstand im Sinne des Art. 21 EuGVÜ/Art. 27 EuGV-VO, wobei zu beachten ist, dass beide Normen unterschiedliche Regelungsziele verfolgen und daher nur bedingt vergleichbar sind. Der EuGH ist zu Art. 21 EuGVÜ der Ansicht, „derselbe Anspruch“6856 beinhalte bei autonomer Auslegung den gleichen Klagegrund. Dieser umfasse den Lebenssachverhalt und die Rechtsvorschrift, auf die die Klage gestützt wird ${ }^{857}$ Für den Streitgegenstand im Sinne des Art. 21 EuGVÜ/ Art. 27 EuGV-VO kommt es demnach nicht primär auf den Antrag an. Vielmehr muss beurteilt werden, ob zwei Verfahren bei wertender Betrachtung denselben Anspruch betreffen. Dabei soll es keinen Unterschied machen, ob Zahlung, oder negative Feststellung verlangt wird. ${ }^{858}$ Dies kann natürlich nicht für den Streitgegenstand im Sinne des Art. 27 Nr. 2 EuGVÜ/ Art. 34 Nr. 2 EuGV-VO gelten, da dabei gerade auch der Antrag von entscheidender Bedeutung ist.

Für den Inhalt des einleitenden Schriftstücks, wie ihn Art. 27 Nr. 2 EuGVÜ/ Art. 34 Nr. 2 EuGV-VO bestimmen, kommt es demnach auf den Streitgegenstand an, der sich aus Antrag und dazugehörigem Lebenssachverhalt zusammensetzt, da es dem Beklagten nur dann möglich ist, seine Verteidigung vorzubereiten. Mehr als die Individualisierung des Streitgegenstandes ist jedoch nicht erforderlich. ${ }^{859}$

\section{b. Entgegenstehende Rechtshängigkeit}

Art. 21 EuGVÜ/Art. 27 EuGV-VO bestimmt die Wirkungen der Rechtshängigkeit für Klagen wegen desselben Anspruchs. Wie bereits ausgeführt, ist „derselbe Anspruch“" autonom und weit auszulegen. ${ }^{860}$ Der Zeitpunkt, auf den abzustellen ist, war für Art. 21 EuGVÜ nach der jeweiligen lex fori des Gerichts zu bestimmen, bei dem die Klage anhängig gemacht wurde. ${ }^{861}$ Diesen Zeitpunkt regelt Art. 30 EuGV-VO nun positiv: Für die Frage der Rechtshängigkeit kommt es auf den Zeitpunkt der Einreichung der Klage an, soweit der Kläger alle Maßnahmen getroffen hat, damit das Schriftstück dem Beklagten unverzüglich zugestellt werden kann. In Systemen, in denen das Schriftstück erst dem Beklagten zuge-

\footnotetext{
${ }^{853}$ EuGH Klomps/Michel Slg. 1981, S. 1593

${ }^{854}$ Kropholler, Art. 27 Rn. 25

${ }^{855}$ EuGH Gubisch Maschinenfabrik/Palumbo Slg. 1987, S. 4861, 4875; EuGH Tatry/Maciej Rataj Slg. 1994 I, S. 4275, Nr. 30; umfassend dazu Stafyla, S. 17 ff.

${ }^{856}$ Art. 21 ff. EuGVÜ; 27 ff. EuGV-VO

${ }^{857}$ EuGH Gubisch Maschinenfabrik/Palumbo Slg. 1987, S. 4861, 4875; EuGH Tatry/Maciej Rataj Slg. 1994 I, S. 4275, Nr. 30

${ }^{858}$ EuGH Tatry/Maciej Rataj Slg. 1994 I, S. 4275, Nr. 30

${ }^{859}$ Dies ergibt sich aus der Tatsache, dass der Mahnbescheid ein verfahrenseinleitendes Schriftstück im Sinne des EuGVÜ/der EuGV-VO ist. Vgl. EuGH Klomps/Michel Slg. 1981, S. 1593

${ }^{860}$ Kritisch aus englischer Sicht: Cheshire and North, S. 252

${ }^{861}$ EuGH Zelger/Salinitri Slg. 1984, S. 2397
} 
stellt werden muss, kommt es jetzt auf den Zeitpunkt an, an dem die Klage der zustellenden Behörde übergeben wurde, vorausgesetzt, der Kläger hat alles getan, damit das Schriftstück auch bei Gericht eingeht. Diese Neuregelung hat nicht nur für das englische, sondern auch für das deutsche Verfahren Bedeutung, da nach der bisherigen Rechtslage in beiden Ländern für das lis alibi pendens auf den Zeitpunkt der Zustellung an den Beklagten abgestellt wurde. Die Regel der EuGV-VO hat den Vorteil, dass der jeweils frühstmögliche Zeitpunkt über die Rechtshängigkeit entscheidet und es nicht darauf ankommt, wie lange die Weiterleitung des Schriftstücks dauert, solange der Kläger nur seinen Pflichten nachgekommen ist. ${ }^{862}$ Werden konkurrierende Verfahren an zwei Gerichte gebracht, muss das zweitangerufene Gericht sein Verfahren bis zu einer Entscheidung des erstangerufenen Gerichts über seine Zuständigkeit aussetzen, Art. 21 I EuGVÜ/ Art. 27 I GV-VO. Steht die Zuständigkeit des erstangerufenen Gerichts fest, erklärt sich das andere Gericht für unzuständig, Art. $21 \mathrm{II}$ EuGVÜ/ Art. 27 II EuGV-VO.

\section{Rechtsvergleichende Betrachtung}

Das EuGVÜ/die EuGV-VO unterscheidet sich von den anderen dargestellten Prozessordnungen und Entwürfen vor allem durch die Tatsache, dass es keine Prozessordnung darstellt, die das gesamte Verfahren regelt, sondern ausschließlich in seinem Anwendungsbereich andere Prozessordnungen modifiziert. Daher kann und muss sich ein Rechtsvergleich hier auf die zwei Punkte beschränken, an denen das EuGVÜ/die EuGV-VO die Verfahrenseinleitung berührt.

Das einleitende Schriftstück im Sinne des Art. 27 Nr. 2 EuGVÜ/ Art. 34 Nr. 2 EuGV-VO muss ein Verfahren einleiten, das zum Erlass eines vollstreckbaren Titels führen kann. Insofern deckt sich die Definition mit der deutschen Rechtslage bzw. ist notgedrungen weiter, als die ZPO es vorsieht, da das EuGVÜ/die EuGV-VO alle einleitenden Schriftstücke aus allen möglichen Rechtsordnungen erfassen will.

Inhaltlich ist es für das EuGVÜ/die EuGV-VO entscheidend, dass sich der Beklagte aufgrund des Schriftstückes verteidigen kann. Daher kommt es insoweit nur auf die Interessen des Beklagten an. Die inhaltlichen Anforderungen beschränken sich dementsprechend neben der immer erforderlichen Parteinämlichkeit auf den Streitgegenstand. Dieser muss aus Antrag und Lebenssachverhalt bestehen, wobei es beim Antrag darauf ankommt, dass der Beklagte erkennen kann, in welcher Höhe ihm eine Verurteilung droht. Daher muss die ungefähre Angabe der Klagesumme erfolgen. Dies deckt sich im Ergebnis mit der deutschen und englischen Regelung. Allerdings liegen andere Regelungszwecke zu Grunde. Im deutschen Recht ist die Angabe einer ungefähren Summe für die Streitwertbestimmung ${ }^{863}$ unerläßlich. In England erfolgt danach die Einteilung in die verschiedenen Tracks. ${ }^{864}$ Dabei handelt es sich jeweils um Vorschriften, die den Verfahrensablauf garantieren. Die zusätzliche Funktion des Beklagtenschutzes tritt dabei in den Hintergrund, zumal sich der Beklagte nicht darauf verlassen kann, dass das Gericht nicht über die Klägervorstellungen hinausgeht. ${ }^{865}$

\footnotetext{
${ }^{862}$ Kropholler, Art. 30 Rn. 2

${ }^{863}$ Die Angabe ist gleichermaßen für den Zuständigkeits-, Kosten- und Rechtsmittelstreitwert von Bedeutung.

${ }^{864}$ S.o. B.III.2.a.aa.

${ }^{865} \mathrm{Im}$ deutschen Verfahren gilt das nur für den nach oben nicht begrenzten unbezifferten Klageantrag, im neuen englischen Recht ist das Gericht grundsätzlich nicht mehr absolut an den Antrag gebunden. S.o. B.III.2.a.aa.
} 
Bezüglich des Sachvortrages reicht für das EuGVÜ/die EuGV-VO die Angabe von Tatsachen zur Individualisierung des Streitgegenstandes aus. Auf substantiierten umfassenden Vortrag kommt es nicht an. Es wäre auch kontraproduktiv, im Rahmen des EuGVÜ/der EuGV-VO engere Voraussetzungen zu schaffen, als dies nach den nationalen Rechten der Fall ist, da das EuGVÜ/die EuGV-VO alle einleitenden Schriftstücke erfassen möchte.

Im Hinblick auf die Klagekonkurrenz ist einmal die weite Fassung des Streitgegenstandsbegriffes auffällig. Die autonome europäische Definition geht von einem sehr pragmatischen Standpunkt aus und lässt es demnach auf den Antrag im Einzelnen nicht ankommen. Dies ist vor dem Ziel, widersprüchliche Urteile zu vermeiden, verständlich. Sowohl die materielle Gerechtigkeit als auch die Prozessökonomie sprechen für diesen weiten Begriff. Das deutsche Recht hingegen wählt einen sehr formalen Standpunkt. Nur soweit sich auch die Anträge decken oder zumindest einer der Anträge im anderen als Minus enthalten ist, ${ }^{866}$ greift $\S 261$ III Nr. 1 ZPO. Das englische Recht wählt einen gänzlich anderen Weg und stellt die Entscheidung, ob eine anderweitige Rechtshängigkeit dem Verfahren entgegensteht, nach Forum-non-conveniens-Gesichtspunkten in das Ermessen des Gerichts.

Der pragmatische Ansatz des EuGH scheint der zu formalistischen deutschen Lösung überlegen zu sein. Ein Vergleich mit dem englischen Recht ist ohne eine umfassende Betrachtung des Instituts Forum-non-conveniens nicht möglich. ${ }^{867}$

In Bezug auf den Zeitpunkt der Rechtshängigkeit stellt Art. 30 EuGV-VO einen Fortschritt gegenüber dem EuGVÜ dar. Die Entscheidung der lex fori des angerufenen Gerichts zu überlassen, wirkte eher hemmend auf die Vereinheitlichungsbestrebungen. Die nun gewählte Form verdient Beifall. Der frühstmögliche Zeitpunkt wurde gewählt und die Fortdauer der Wirkungen hängt ausschließlich vom Verhalten des Klägers ab. Versäumnisse des Gerichts oder von Behörden können dem Kläger nicht zum Nachteil gereichen. Der Beklagte wird dabei auch nicht unangemessen benachteiligt. Die englische Regel zur Rechtshängigkeit ab Zustellung im Rahmen des EuGVÜ ist damit hinfällig. Die Regelung der EuGV-VO ist dem deutschen Recht überlegen, da Versäumnisse des Gerichts nicht zu Nachteilen für den Kläger führen. Sofern die EuGV-VO anwendbar ist, ändert sich damit die deutsche Rechtslage. Es wäre wünschenswert, wenn auch die anderen Wirkungen der Rechtshängigkeit, insbesondere die Hemmung der Verjährung auf diesen frühen Zeitpunkt verlagert werden könnten. $\S 167$ n.F. ZPO ist insoweit unbefriedigend, da auch ohne Verschulden des Klägers eine Klage möglicherweise nicht mehr „,demnächst“ zugestellt wird. Art. 30 EuGV-VO scheint vor diesem Hintergrund auch sinnvoller als Art. 2.2.2.1 des Storme-Entwurfs, der eine absolute Drei-Monats-Frist festlegt.

\footnotetext{
${ }^{866}$ Wie der Feststellungsantrag im Verhältnis zum Leistungsantrag.

${ }^{867}$ Dies würde den Rahmen der Arbeit sprengen.
} 


\section{E. Abschließende Betrachtung der Entwicklungstendenzen}

Bei abschließender Betrachtung der verglichenen Systeme stehen vier Kernbereiche im Fokus des untersuchten Themas:

1. Welchem System folgt die Verfahrenseinleitung?

2. Zu welchem Zeitpunkt entstehen die Rechtshängigkeitsfolgen?

3. Welchen Inhalt muss das einleitende Schriftstück haben?

4. Wie erfolgt die Zustellung?

\section{System der Verfahrenseinleitung}

Bei der Verfahrenseinleitung sind zwei grundlegende Systeme zu unterscheiden. Zum einen kann das Verfahren durch Einreichen einer Klage bei Gericht geschehen. Diese Klage wird sodann dem Beklagten durch das Gericht oder den Kläger zugestellt. Zum anderen kann die Klage unmittelbar dem Beklagten zugestellt werden. Gleichzeitig oder im Anschluss daran wird die Klage dem Gericht zugeleitet.

Sowohl das deutsche, als auch das englische Recht folgen dem ersten Konzept. Schon im Mittelalter wurde in beiden Rechtsordnungen die Privatladung durch einen königlichen Ladungsbefehl (Bannitio, Writ) ersetzt. Die Trennung von Klage inklusive Klagebegründung und Ladungsbefehl wurde im deutschen Recht durch die preußischen Reformen und die Vorgänger der ZPO im 19. Jahrhundert aufgehoben. Im englischen Recht besteht sie prinzipiell fort, wobei es sich nur noch um eine historisch bedingte Eigenheit handelt. Ihre Funktion als bedingten hoheitlichen Leistungsbefehl an den Beklagten kann die Ladung ohnehin nicht mehr erfüllen, da der Kläger dem Beklagten die particulars of claim innerhalb von zwei Wochen zustellen oder diese gleich auf der claim form eintragen muss. Im letzten Fall ist die Trennung von Ladung und Klagebegründung ohnehin aufgehoben, im ersten Fall besteht sie nur noch theoretisch fort. Von Bedeutung ist daher nur noch, dass das englische Verfahren mit Hilfe eines Formblattes eingeleitet wird. Damit wird sichergestellt, dass der Kläger keine erforderlichen Angaben vergisst.

Aus Gründen der Einfachheit und Prozessökonomie scheint die Einleitung mittels eines einzigen Schriftsatzes vorzugswürdig. Die Trennung von Ladungsbefehl und Klagebegründung ist überholt. Welchen Inhalt ein solches Schriftstück haben sollte, bleibt noch zu bewerten. Die Idee des Formblattes hat sehr alte historische Wurzeln, könnte aber in naher Zukunft wieder aktuell werden, nämlich bei der Einleitung über neue Medien. Hält man die Klageeinleitung online für ein erstrebenswertes Ziel, sollte man über ein Online-Formular nachdenken, das auf der Homepage des zuständigen Gerichts ausgefüllt und abgeschickt werden kann. ${ }^{868} \mathrm{Ob}$ ein solches Verfahren für normale Klagen sinnvoll ist, ist allerdings zweifelhaft. Anders mag dies im Mahnverfahren aussehen, wo der formalisierte Rechtsverkehr schon weitgehend Realität ist.

Die Einreichung der Klage als elektronisches Dokument sollte hingegen in näherer Zukunft schon möglich sein. Ob dann auch elektronische Akten geführt werden sollten und ob dadurch der Arbeits- und Papieranfall verringert oder eher vergrößert wird, bleibt abzuwarten. Die Einreichung der Klage, und logischerweise folgend, auch die Korrespondenz über elektronische Medien, wie sie zur

${ }^{868}$ Die Klageerhebung durch Ausfüllen eines Formulars führt allerdings zu Verifizierungsproblemen, da eine Signatur mitgesandt werden müsste. 
Zeit beim BGH als Modellversuch läuft, wird sicher mittelfristig den Postweg ablösen.

\section{Rechtshängigkeitsfolgen}

Bei den Folgen der Rechtshängigkeit muss nach deutschem Rechtsverständnis zwischen materiellen und prozessualen Rechtsfolgen unterschieden werden. Bei den materiellen Rechtsfolgen ist insbesondere die Verjährung zu nennen, bei den prozessualen die entgegenstehende Rechtshängigkeit. Das englische Recht macht diese Unterscheidung nicht, da es die Verjährung als prozessuales Klagehindernis begreift.

Die deutsche Lösung über $\S 167$ n.F. ZPO lässt die Rechtshängigkeit erst mit Zustellung der Klage an den Beklagten eintreten und verlegt alle fristgebundenen Rechtshängigkeitswirkungen auf den Anhängigkeitszeitpunkt (Einreichung der Klage bei Gericht) vor, wenn die Zustellung demnächst erfolgt. Dieses System stellt sich im internationalen Vergleich als unnötig kompliziert dar. Es leidet insbesondere unter zwei Schwachpunkten. Erstens fehlt der Regelung über die Zustellung „demnächst“ die zu wünschende Rechtssicherheit. Es ist sinnvoll, dem Kläger aufzuerlegen, alles zu tun, was er tun muss, damit die Klage zugestellt werden kann. Ein zusätzliches Zeitelement, nämlich die Frage, wann eine Zustellung noch als „,demnächst“ betrachtet werden kann, bringt jedoch erhebliche Rechtsunsicherheit, insbesondere, wenn die Beurteilung des Zeitraums einzelfallabhängig ist. ${ }^{869}$ Die Regelung schützt zwar das Vertrauen des Beklagten, seine Rechtsposition nicht zeitlich unbegrenzt wieder verlieren zu können, leidet aber unter der Unbestimmtheit des Zeitelements. ${ }^{870}$ Zweitens erfolgt die Vorverlegung der Wirkungen nach $\S 167$ ZPO nicht für alle Rechtshängigkeitsfolgen, sondern nur für die Fristwahrung und Verjährungsunterbrechung. Damit herrschen im selben Regelungsbereich verschiedene Regimes.

Die englische Lösung bietet sich allerdings auch nicht als Muster an. Die Verjährungsunterbrechung erfolgt zwar grundsätzlich mit Ausstellung der Claim form, nicht jedoch, wenn diese nicht innerhalb von vier Monaten ${ }^{871}$ zugestellt wird. Diese Regelung ist erkennbar noch auf die Parteizustellung zugeschnitten. Zur nunmehr zur Regel gemachten Amtszustellung passt sie nicht, da offen bleibt, was passiert, wenn das Gericht die Claim form nicht innerhalb von vier Monaten zustellt. Bei gerichtsinternen Problemen ist der Kläger mithin schutzlos.

Ähnliche Inkonsistenzen ergeben sich beim lis alibi pendens. Die entgegenstehende Rechtshängigkeit als absolutes Klagehindernis kennt das englische Recht nicht. Vielmehr entscheidet das Gericht unter abuse-of-process- (national) oder forum-non-conveniens-Gesichtspunkten (international) über die Zulässigkeit der Klage. Solange das EuGVÜ galt, war der entscheidende Zeitpunkt für Art. 21 EuGVÜ̉ die Zustellung an den Beklagten. ${ }^{872}$ Diese Rechtslage ist mit der EuGV-VO überholt. ${ }^{873}$ Gleiches gilt für das deutsche Recht, soweit die EuGV-VO Anwendung findet.

Für die entgegenstehende Rechtshängigkeit ist es sinnvoll, den Rechtshängigkeitszeitpunkt so früh wie möglich festzulegen, wie dies die EuGV-VO tut. Weder die deutsche Regelung mit ihrem unbestimmten Zeitelement, noch die Lösung, die

\footnotetext{
${ }^{869}$ BGH NJW 1999, S. 3125; Zöller/Greger $\$ 167$ n.F. Rn. 10

${ }^{870}$ Zöller/Greger $§ 167$ n.F. Rn. 1

${ }^{871}$ Bei Zustellung ins Ausland sechs Monate.

${ }^{872}$ Dresser (UK) Ltd v. Falcongate [1992] All ER 450 (C.A.); Neste Chemicals v. DK Line [1994] Lloyd's Rep. 6

${ }^{873}$ S.o.D.III.1.b.
} 
der Storme-Entwurf vorsieht, wonach eine absolute Drei-Monats-Frist für die Zustellung über die Wirkungen bestimmt, kann überzeugen. Die Lösung, die im Rahmen der EuGV-VO für intereuropäische Verfahren ohnehin gilt, sollte auch das Ziel einer möglichen Neuregelung sein. Um der Rechtsklarheit Willen sollten alle prozessualen, sowie fristwahrenden Rechtshängigkeitswirkungen auf diesen frühen Zeitpunkt verlagert werden. Anderes gilt nur für die materiellen Wirkungen, soweit es dabei auf die Kenntnis des Beklagten von der Rechtshängigkeit ankommt. ${ }^{874}$

\section{Inhalt des einleitenden Schriftstücks}

Der Rechtsvergleich hat gezeigt, dass aufgrund der verschiedenen Systeme unterschiedliche Dokumente zur Verfahrenseinleitung benutzt werden. Dies ist zum einen die Klageschrift nach deutschem Recht, der Ladungsbefehl (Writ, Claim form) mit oder ohne Klagebegründung nach englischem Recht, die Klageschriften in den PRoTCP und dem Storme-Entwurf sowie der deutsche Mahnantrag und sein Pendant im Storme-Entwurf. Alle haben gemein, dass sie ein Verfahren einleiten, das zum Erlass eines vollstreckbaren Titels führen kann und bei Verteidigung des Beklagten als streitiges Verfahren geführt wird.

Inhaltlich muss das Einleitungsdokument verschiedene Anforderungen erfüllen, die sich zum Teil stark gleichen, mitunter aber auch sehr unterschiedlich ausgestaltet sind. Nur wenn das Schriftstück die inhaltlichen Voraussetzungen erfüllt, liegt eine ordnungsgemäße Einleitung des Verfahrens vor. Erst dann ist das Verfahren rechtshängig (bzw. anhängig, wobei diese Unterscheidung eliminiert werden sollte) und kann die entsprechenden Wirkungen entfalten.

\section{Anforderungen in subjektiver Hinsicht}

In subjektiver Hinsicht fällt insbesondere auf, dass die aktuellen Vorschläge der Storme-Kommission und des ALI/Unidroit auf subjektive Merkmale verzichten. Es ist anzunehmen, dass es sich dabei um bewusste Auslassungen handelt. Das deutsche und englische Recht entsprechen einander in ihrem Regelungsgehalt.

Grundsätzlich müssen die Parteien so bezeichnet werden, dass eine Verwechslung ausgeschlossen werden kann. Üblicherweise sollte dies durch Namen und Anschrift geschehen, in Ausnahmefällen können aber auch weitere Angaben erforderlich oder weniger genügend sein. Hier ist eine flexible pragmatische Lösung gefragt. Dem Kläger sollten so wenig Vorschriften wie möglich gemacht werden, die Folgen mangelhafter Personalisierung müssen aber zu seinen Lasten gehen. Bei nicht prozessfähigen Personen ist der gesetzliche Vertreter aufzuführen. Ebenso ist anzugeben, wenn eine Partei in einer vertretenden Funktion im weitesten Sinne handelt. ${ }^{875}$

Die Angabe des Prozessbevollmächtigten des Klägers sollte nur im Anwaltsprozess zwingend sein und sonst als Zweckmäßigkeitsnorm ausgestaltet werden. In Anbetracht der neuen Medien sollte die Korrespondenz mittels Fax, E-Mail oder entsprechender Medien dann möglich sein, wenn die Parteien oder ihre Vertreter entsprechende Adressen bzw. Nummern angegeben haben. Gibt jemand beispielsweise eine E-Mail-Adresse an, ist davon auszugehen, dass er der Korrespondenz über diese Adresse zustimmt. Die englische Regelung, wonach ein ausdrückliches Einverständnis vorliegen muss, ist insoweit zu restriktiv.

\footnotetext{
${ }^{874}$ Bspw. die verschärfte Haftung, $\S \S 819,989$ BGB.

${ }^{875}$ Z.B. als Partei kraft Amtes, Prozessstandschafter, gesetzlicher oder verfassungmäßig berufener Vertreter.
} 
Des Weiteren muss das einleitende Schriftstück die Bezeichnung des Gerichts enthalten. Weitere Angaben, die die interne Geschäftsverteilung betreffen, sind überflüssig. Die englische Regelung, wonach beim High Court die Abteilung angeführt werden soll, ist ein Relikt aus der Zeit der verschiedenen Gerichtszweige und der Trennung von Gerichten at common law und in equity.

Wird das Verfahren nicht beim Gericht der streitigen Hauptsache begonnen (bspw. im Mahnverfahren), ist das Gericht der Hauptsache anzugeben. Die Angabe des falschen Gerichts sollte zur Verweisung von Amts wegen führen, wobei die Verzögerung und Mehrkosten dem Kläger zur Last fallen.

\section{Anforderungen in objektiver Hinsicht}

Die Anforderungen in objektiver Hinsicht kreisen in allen verglichenen Rechtsordnungen um den Streitgegenstandsbegriff. Dieser wird für das Einleitungsdokument einheitlich als aus Antrag und Lebenssachverhalt bestehend definiert.

Beim Antrag ist auf Genauigkeit Wert zu legen. Zahlungsklagen sind zu beziffern, wobei Ausnahmen zuzulassen sind. Werden unbezifferte Zahlungsanträge gestellt, sollte die ungefähre Größenordnung angegeben werden. Dies ist aus verschiedenen Gründen erforderlich. Zum einen bestimmt sich danach unter Umständen die Zuständigkeit des Gerichts, die Verfahrensart, die Rechtsmittelsumme und die Höhe der Kosten. Zum anderen kann sich der Beklagte auf die Größenordnung einstellen und unter Umständen ein Versäumnisurteil gegen sich ergehen lassen, wobei zu beachten ist, dass das Gericht nach keiner betrachteten Rechtsordnung an die Größenordnung gebunden ist.

Ist der Zahlungsantrag beziffert, sollte er für das Gericht die Höchstgrenze des Urteils darstellen. Die neue englische Regel, wonach das Gericht mehr oder anderes zusprechen kann, als beantragt, widerspricht dem Dispositionsgrundsatz. Soweit rechtspolitischen Erwägungen zur Waffengleichheit der Parteien Rechnung zu tragen ist, lässt sich dies durch die richterliche Hinweispflicht, Prozessleitungsfunktion und gesteigerte Sorgfaltspflichten des Gerichts für anwaltlich nicht vertretene Parteien realisieren. Eine Einschränkung der Dispositionsmaxime ist im Zivilverfahren insoweit abzulehnen.

Das zweite Standbein des Streitgegenstandes ist der Lebenssachverhalt, der dem Anspruch zugrunde liegt. Hier stellt sich die Frage, ob der Kläger in seinem einleitenden Schriftstück nur genug Tatsachen angeben muss, um den Streitgegenstand $\mathrm{zu}$ individualisieren, also dem Beklagten die Identifikation des tatsächlichen Hintergrundes der Klage zu ermöglichen. Andernfalls muss schon das Einleitungsdokument so vollständig und substantiiert wie möglich die Tatsachen enthalten, die die Tatbestandsmerkmale der Anspruchsgrundlage ausfüllen.

Das englische Recht folgt der ersten Variante, allerdings mit der Einschränkung, dass die ausführliche Klagebegründung spätestens in den Particulars of claim innerhalb von 14 Tagen nachfolgen muss. Auch das deutsche Recht verlangt in der Klage selbst nur die Individualisierung des Streitgegenstandes, wobei ein relativ vollständiger Tatsachenvortrag in der Klage die praktische Regel ist. Unklar ist die Regelung im Storme-Entwurf. Obwohl bei der normalen Klageeinleitung vieles darauf hinweist, dass die Identifikation des Streitgegenstandes ausreichen soll, muss bei der Einleitung im Mahnverfahren substantiiert (sogar unter Antritt des Urkundenbeweises) vorgetragen werden.

Nur die PRoTCP folgen der zweiten Variante. Alle Rechtsordnungen erkennen dabei an, dass der vollständige Tatsachenvortrag am Anfang des Verfahrens nicht immer möglich ist. 
Eine moderne Regelung auf Grundlage der verglichenen Rechtsordnungen sollte die Angabe von anspruchsbegründenden Tatsachen schon in der Klageschrift verlangen. Dies ist in Deutschland ohnehin die praktische Regel. In England muss man Claim form und Particulars auch als Einheit begreifen, die unter Umständen zeitlich auseinander gerissen ist. Letzten Endes ist die Unterscheidung von Ladungsbefehl und Klagebegründung nur noch theoretischer Art. Diese Erwägungen werden auch dadurch gestützt, dass eine Wiedereinführung der Schlüssigkeitsprüfung im Mahnverfahren zu wünschen ist, ${ }^{876}$ und dann auch im Mahnantrag substantiierter Tatsachenvortrag erfolgen muss. Die Angabe der anspruchsbegründenden Tatsachen sollte allerdings großzügig gehandhabt werden. Insbesondere dürfen in der Klage noch keine hohen Ansprüche an die Substantiierung gestellt werden, da der Grad der Substantiierung oft erheblich vom Bestreiten des Beklagten abhängt. Erforderlich sollte demnach ein in sich schlüssiger Tatsachenvortrag sein, der geeignet ist, die im Antrag gewünschte Rechtsfolge herbeizuführen. Dabei kann die englische Regelung zu den Particulars of claim als Vorbild dienen. ${ }^{877}$

Beweismittel sollten in der Klage nicht zwingend erforderlich, in der Regel aber zweckmäßigerweise angegeben werden. Dies entspricht der deutschen Rechtslage und der Tendenz im englischen Recht. In England war die Angabe von Beweismitteln im Schriftsatzverfahren bis zur Einführung der CPR 1998 nicht gestattet. Nunmehr können Beweismittel angegeben werden. Die Entwicklung geht demnach eindeutig in diese Richtung. Ähnliche Regelungen finden sich auch in den Diskussionsentwürfen, wonach Beweismittel angegeben werden sollen bzw. müssen, soweit es vernünftigerweise praktikabel ist.

Es sollte den Parteien freistehen, Rechtsausführungen zu machen. In allen verglichenen Prozessordnungen gilt der Grundsatz da mihi factum, dabo tibi ius. Die Pflicht zu Rechtsausführungen, wie sie die PRoTCP vorsehen, widersprechen mithin dem System. Dabei ist allerdings zu beachten, dass die PRoTCP nur für internationale Verfahren gelten, bei denen es in der Regel auch zur Anwendung fremden Rechts kommt. Fremdes Recht sollte grundsätzlich als Recht, nicht als Tatsache behandelt werden und den gleichen Grundsätzen unterliegen wie die lex fori.

\section{Anforderungen in formaler Hinsicht}

In formaler Hinsicht stellt sich die Frage, ob eine Klage mit eigenhändiger Unterschrift versehen sein muss, oder ob sich gegebenenfalls eine Wahrheitsversicherung anbietet, wie sie die CPR 1998 eingeführt haben. Für die Authentifikationsfunktion ist die eigenhändige Unterschrift genügend, aber auch erforderlich. Insofern ist es schwierig nachzuvollziehen, warum nach den CPR 1998 das Unterschriftserfordernis nur für Anwälte gelten soll. Die Unterschrift dient auch dem Zweck, den Unterzeichner als Verantwortlichen für das Schriftstück zu identifizieren.

Die Unterschrift sollte demnach in jedem Fall formales Erfordernis eines einleitenden Schriftstückes sein. Fraglich ist allerdings, ob die Versicherung der Wahrheit auf europäischer Ebene ein sinnvolles Instrument wäre. Das Statement of truth des neuen englischen Zivilprozessrechts erfüllt vor allem zwei Funktionen. Zum einen soll es der Durchsetzung der Wahrheitspflicht dienen. Zum anderen soll es verhindern, dass Ansprüche oder Verteidigungslinien aus rein taktischen

\footnotetext{
${ }^{876}$ S.o. D.I.2.

${ }^{877}$ S.o. B.III.2.a.bb.
} 
Erwägungen aufrecht erhalten werden. Dementsprechend strikt sind auch die drohenden Sanktionen. Fehlt das Statement of truth, kann im schlimmsten Fall die Nichtzulassung des Schriftsatzes drohen. Wurde das Statement wider besseres Wissen abgegeben, macht sich der Erklärende wegen Contempt of Court strafbar. Obwohl die Vorschriften auf den ersten Blick attraktiv erscheinen, muss ihre Wirksamkeit in Frage gestellt werden. Zum einen ist äußerst fraglich, ob die Gerichte bereit sind, nach Schließen einer Akte die Strafverfolgung zu betreiben. ${ }^{878}$ Zum anderen eröffnet die drohende Strafbarkeit dem Gegner die Möglichkeit, die Anzeige zu missbrauchen, um zusätzlichen Druck auszuüben oder Rache zu nehmen. ${ }^{879}$

\section{Zustellung}

Bei der Zustellung ergeben sich wiederum drei Problemkreise:

1. Wer soll die Zustellung vornehmen?

2. Auf welche Art und Weise soll zugestellt werden?

3. Was muss zugestellt werden?

\section{Amts- oder Parteizustellung?}

Sowohl im deutschen als auch im englischen Recht lässt sich die eindeutige Tendenz von der Parteizustellung weg und zur Amtszustellung hin feststellen. Der deutsche Gesetzgeber führte die Amtszustellung bereits 1976 (wieder) ein. In England herrschte nur in den Anfängen des Writ-Systems eine Art Amtszustellung, da die Writs dem Beklagten in der Regel vom Sheriff mitgeteilt wurden. Unter den RSC/CCR wurde dann die Parteizustellung üblich. Erst seit Einführung der CPR 1998 obliegt die Zustellung grundsätzlich wieder dem Gericht, es sei denn, der Kläger will selbst zustellen. Eine moderne Regelung sollte diesen Tendenzen folgen und die Amtszustellung vorschreiben. Dies harmoniert auch am besten mit der vorgeschlagenen Regelung bezüglich der Rechtshängigkeitswirkungen.

\section{Art der Zustellung}

Bei der Zustellung ist in Anbetracht der modernen Kommunikationsmedien ein ausgewogener Ausgleich zwischen herkömmlichen und modernen Zustellungsvarianten $\mathrm{zu}$ finden.

Grundsätzlich sollte die persönliche Zustellung die Regel bleiben. Aus Gründen der Einfachheit wird man die Zustellung per Einschreiben mit Rückschein, so wie sie auch in der EuZustVO vorgesehen ist, auch im nationalen Verfahren verfügbar machen müssen. Moderne Zustellungsmethoden sollten grundsätzlich zur Verfügung stehen, allerdings unter dem Vorbehalt, dass sich der Empfänger mit der Zustellung auf diesem Wege einverstanden erklärt hat. Dieses Einverständnis sollte bei Angabe von Faxnummer, E-Mail-Adresse oder ähnlichem vermutet werden.

Nur wenn die persönliche oder postalische Zustellung keinen Erfolg hat, sollte die Ersatzzustellung, und wenn diese auch misslingt, die öffentliche Zustellung zugelassen werden. Bei diesen subsidiären Zustellungsformen, insbesondere der öffentlichen Zustellung, geht die Tendenz zu einem gesteigerten Ermessen des Gerichts. In England ist das Gericht insoweit gänzlich frei, in Deutschland ist das gerichtliche Ermessen zuletzt durch das ZustRG vom 25.06.2001 zusätzlich ge-

\footnotetext{
${ }^{878}$ Um dies beurteilen zu können müsste man zum Vergleich die Anzahl tatsächlich eingeleiteter Strafverfahren wegen uneidlicher Falschausage als Zeuge heranziehen.

${ }^{879}$ Kritisch auch Blackstone's Civil Practice, Rn. 23.14
} 
stärkt worden, indem bei der öffentlichen Zustellung die Veröffentlichung im Bundesanzeiger nur noch notwendig sein soll, wenn das Gericht dies anordnet, $\S \S 187,186$ ZPO. Auch eine neue Regelung sollte neben den Regelzustellungsformen und der subsidiären Ersatzzustellung die öffentliche Zustellung als ultima ratio in das Ermessen des Gerichts stellen.

$\mathrm{Ob}$ bei der Zustellung über elektronische Medien eine verkörperte Kopie folgt, sollte man den Parteien überlassen, soweit diese etwas zustellen. Bei Zustellung durch das Gericht ist eine derartige Doppelzustellung wohl in der Regel überflüssig, da durch die gerichtliche Zustellung keine Fristen gewahrt werden müssen.

Wer die Zustellung bewusst vereitelt, muss sie als erfolgt gegen sich gelten lassen, wie auch im deutschen und englischen Recht.

Die Zustellung ins Ausland sollte grundsätzlich der ZustVO für EU-Länder und ansonsten dem vergleichbaren HZustÜ folgen. Weitere Regime für Drittländer verkomplizieren die Rechtslage unnötig.

\section{Zuzustellende Dokumente}

Zunächst ist dem Beklagten die Klage inklusive Begründung zuzustellen. Mit der Klage sollten zum einen alle gerichtlichen Verfügungen und gesetzlichen Fristen mitgeteilt werden, zum anderen der Beklagte über den weiteren Verlauf des Verfahrens, insbesondere bei Säumnis oder Verspätung aufgeklärt werden. Die Frist zur Anzeige der Verteidigungsbereitschaft ist mit zwei Wochen zu bemessen, für die Klageerwiderung sind weitere zwei Wochen anzusetzen. Dies entspricht sowohl englischer als auch deutscher Rechtslage. Eine Verlängerung der Frist sollte in das Ermessen des Gerichts gestellt werden, vor allem, wenn die Zustellung ins Ausland erfolgt.

Bei der Zustellung ins Ausland sind allen Schriftstücken Übersetzungen in die offizielle Landessprache beizufügen. ${ }^{800}$

\footnotetext{
${ }^{880}$ Bei mehreren offiziellen Landessprachen ist die in der Region übliche Amtssprache zu wählen, bei mehreren üblichen Amtssprachen steht die Auswahl dem Kläger frei, der die Übersetzungen bereitstellen muss.
} 


\section{F. Fazit}

Die Untersuchung der verschiedenen Prozessrechtsordnungen hat gezeigt, dass die Verfahrenseinleitung indikativ ist für Veränderungen im System, die ihrerseits gesellschaftlichen Entwicklungen und Bedürfnissen folgen.

Sowohl das englische als auch das deutsche Prozessrecht kommen ursprünglich aus dem germanischen Recht, in dem kein eigenständiger Zivilprozess existierte. Erst mit steigendem Herrschaftsanspruch der Könige entwickelte sich auch ein Zivilverfahren, das ursprünglich adversativen Charakters war.

Dieses blieb in England weitgehend von äußeren Einflüssen verschont, wohingegen sich in Deutschland zunehmend der Staat des Verfahrens bemächtigte, bis es im Absolutismus zum inquisitorischen Aktenprozess kam.

In der zweiten Hälfte des 19. Jahrhunderts wurde der Zivilprozess in Deutschland mit der ZPO und in England durch die endgültig Abschaffung der Forms of action, die ihre Verkörperung in den Writs gefunden hatten, im Zuge des Liberalismus von staatlichem Einfluss weitgehend befreit.

Die dadurch entstandenen Systeme sahen sich im 20. Jahrhundert identischen gesellschaftlichen Strömungen ausgesetzt. Das führte zu einer sozialstaatlichen Ausprägung der ZPO und zur ebenfalls sozialstaatlichen Neuordnung des englischen Verfahrens in den CPR 1998.

Die neuen Systeme in beiden Ländern unterscheiden sich in der Verfahrenseinleitung nur noch vergleichsweise marginal. Auch bei elementaren Grundprinzipien, wie der Einschränkung der Dispositions- zugunsten einer Kooperationsmaxime, verschwinden die Unterschiede.

Dies liegt primär an der gesellschaftlichen Entwicklung, die sich in Deutschland und England angleicht. Weiterhin werden die Zielvorgaben in zunehmendem Maße in Brüssel gesetzt. Durch die starken Rechtssetzungsaktivitäten in Verbraucherschutzangelegenheiten wird der Zivilprozess auch zunehmend von diesem Gedanken erfasst. Dies schlägt sich beispielsweise in Verbrauchergerichtsständen oder in Einschränkungen bei Gerichtsstandsvereinbarungen, an denen ein Verbraucher beteiligt ist, nieder.

Die Entwicklungen zeigen, dass ein moderner Zivilprozess dem gesteigerten Interesse des Bürgers als Verbraucher an staatlicher Garantie eines fairen Verfahrens Rechnung tragen muss. Dabei sollten aber Grundkonzepte des Zivilverfahrens als Streit „unter gleichen“, wie beispielsweise die Dispositionsmaxime, nicht aufgegeben werden. Auch wenn der Bürger als Verbraucher grundsätzlich einen Fürsorgeanspruch an den Staat hat, darf dies nicht dazu führen, dass man in der Tradition des preußischen Wohlfahrtsstaats den Prozess den Parteien entzieht. Daher ist insoweit ein sensibler Ausgleich gefragt, nicht zuletzt, da Wirtschaftsunternehmen oft ganz andere Erwartungen an einen Prozess haben als Verbraucher.

Diesen unterschiedlichen Bedürfnissen und Erwartungen versuchen die PRoTCP Rechnung zu tragen, indem sie ausschließlich bei geschäftlichen Transaktionen, also gerade nicht inVerbrauchersachen, Anwendungen finden sollen. Auch wenn die PRoTCP in den Einzelregelungen noch nicht ausgereift erscheinen, basieren sie insoweit auf einem interessanten Grundgedanken.

In gleichem Maße, in dem im materiellen Zivilrecht ein „Verbraucherrecht“ und ein „Recht für Nicht-Verbraucher" entsteht, ${ }^{881}$ wird man in Zukunft über einen „Verbraucherprozess ${ }^{\star 682}$ nachdenken müssen. Ein solcher könnte, sozialstaatlichen Grundsätzen folgend, den Bedürfnissen des Bürgers gerecht werden. Parallel

\footnotetext{
${ }^{881}$ Insbesondere auf EU-Ebene zeichnet sich eine derartige Tendenz eindeutig ab.
} 
dazu müsste man Nicht-Verbrauchern ein Verfahren zur Verfügung stellen, das ihren Bedürfnissen entsprechend weitgehend dereguliert in den Händen der Parteien bleibt.

${ }^{882}$ Erste Ansätze dazu finden sich z.B. bereits in $§ \S 29$ c ZPO (Gerichtstand), 1031 V ZPO (Form der Schiedsvereinbarung), Art. 15-17EuGV-VO 


\section{Literaturverzeichnis}

The American Law Institute and The International Institute for the Unification of Private Law

Principles and Rules of Transnational Civil Procedure, Discussion Draft No. 3, Philadelphia, Rom 2002

Anders, Monika/ Gehle, Burkhard

Das Assessorexamen im Zivilrecht, 8. Aufl., Düsseldorf 2005

Baker, John H.

An Introduction to English Legal History, 3. ed., London 1990

Baker, John H./ Milsom, S.F.C

Sources of English Legal History, Private Law to 1750, London 1986

Barnard, David/ Houghton, Mark

The New Civil Court in Action, London 1993

Baumbach, Adolf/ Lauterbach, Wolfgang/Albers, Jan/ Hartmann, Peter

Zivilprozeßordnung, 62. Aufl., München 2006

Baumgarten, Thomas

Der richtige Kläger im deutschen, französischen und englischen Zivilprozeß, Frankfurt am Main 2001

Behrends, Okko

Römischrechtliche Exegese: Das deliktische Haftungssystem der Lex Aquilia, in: JuS 1985, S. 878 ff.

Bergerfurth, Bruno

Der Zivilprozeß, 5. Aufl., Freiburg 1985

Bethmann-Hollweg, Moritz A. von

Der Civilprozeß des gemeinen Rechts in geschichtlicher Entwicklung, Bände I und IV, Bonn 1868

Bettermann, Karl August

Hundert Jahre Zivilprozeßordnung, in: ZZP 91 (1978), S. 365 ff. 
Bieresborn, Dirk

Klage und Klageerwiderung im deutschen und englischen Zivilprozeß, Frankfurt am Main 1999

Bigelow, Melville Madison

History of Procedure in England from the Norman Conquest, London 1880

Blackstone, William

Commentaries on the Laws of England, Vol. III, 10. ed., London 1787

Blackstone's Civil Practice 2001

Plant, Charles (Editor-in-Chief); Rose, William (Editor); Sime, Stuart; French, Derek; 2. ed., London 2001

Blackstone's Guide to the Civil Procedure Rules

Plant, Charles (Editor-in-Chief); Rose, William (Editor); London 1999

Blumenwitz, Dieter

Einführung in das Anglo- Amerikanische Recht, 7. Aufl., München 2003

Boockmann, Andrea

Geistliche und weltliche Gerichtsbarkeit im mittelalterlichen Bistum

Schleswig, Neumünster 1967

Bowen, Charles S. C.

The Administration of the Law from 1837 to 1887, in: The Reign of Queen Victoria, A Survey of Fifty Years of Progress; Thomas Humphrey Ward (Editor), London 1887

Bramley, Michelle/ Gouge, Anna

The Civil Justice Reforms One Year On - Freshfelds Assess Their Progress; London 2000

Briegleb, Hans Karl

Einleitung in die Theorie der summarischen Processe, Leipzig 1859, Nachdruck Frankfurt am Main 1969

Broggini, Gerardo

Besprechung von Hans Peter, Actio und Writ, in: ZRG Rom. 75 (1958),

S. $441 \mathrm{ff}$. 
Brunner, Heinrich

Deutsche Rechtsgeschichte, Band I und II, 2. Aufl. Berlin 1928, Neudruck 1958

ders.

Die Entstehung der Schwurgerichte, Berlin 1872

Bücker, Ursula E.

Mündliche und schriftliche Elemente und ihre rechtsgeschichtlichen Hintergründe im englischen Erkenntnisverfahren, Berlin 1978

Bunge, Jürgen

Institutionen des Englischen Zivilprozesses in: ZZP 92 (1979), S. 358 ff.

ders.

Das untere Richterpersonal und die Richtergehilfen am englischen High

Court of Justice, Berlin 1973

ders.

Zivilprozess und Zwangsvollstreckung in England, Berlin 1995

Caenegem, Raoul C. van

History of European Civil Procedure, in: Encyclopedia of Comparative Law Vol. 16 Chapter 2

ders.

Judges, Legislators \& Professors, Cambridge 1987

Casson, David B/ Dennis, Ian H.

Modern Developments in the Law of Civil Procedure, London 1982

Cheshire and North

North, Sir Peter M./ Fawcett, J.J.

Cheshire and North's Private International Law, 13. ed. London 1999

Coing, Helmut

English Equity and the Denunciatio Evangelica of the Canon Law, in:

LQR 71 (1955) S. 223 ff. 
ders.

Handbuch zu den Quellen und Literatur der neuen europäischen Privatrechtsgeschichte, Band III 2. Teilbd., München 1982

Conrad, Hermann

Deutsche Rechtsgeschichte Band II, 2. Aufl., Karlsruhe 1966

Cohn, Ernst J.

Die Lehre vom Schriftsatz nach englischem Recht, in: ZZP 73 (1960) S. 324

Coote, Henry C.

The Practice of the High Court of Admiralty of England, 2. ed., London 1868

Curzon, Leslie B.

English Legal History, 2. ed., Plymouth 1979

Dahlmanns, Gerhardt J.

Der Strukturwandel des deutschen Zivilprozesses im 19. Jahrhundert, Aalen 1971

Damrau, Jürgen

Die Entwicklungen einzelner Prozeßmaximen seit der Reichszivilprozeßordnung von 1877, Paderborn 1975

Dannreuther, Dieter

Der Zivilprozeß als Gegenstand der Rechtspolitik im Deutschen Reich 1871 - 1945, Frankfurt am Main 1987

Day, John C.

The Common Law Procedure Acts and other Statutes relating to the Practice of the superior Courts of Common Law and the Rules of Court, 4. ed., London 1872

Dreymüller, Dagmar

Die Reform des englischen Zivilprozessrechts - Eine Abkehr von fundamentalen Grundsätzen des englischen Zivilprozessrechts unter Annäherung an den deutschen Zivilprozess? in: ZVglRWiss 101 (2002), S. 471 ff. 
dies.

Der Zeugenbeweis im Zivilprozess im common law und im deutschen Recht, Diss. Münster 2000

Durantis, Wilhelm

Speculum Judiciale To. I. und II., Neudruck der Baseler Ausgabe von 1574, Aalen 1975

Eckhardt, Karl August (Hrsg. und Übersetzer)

Lex Salica, Pactus Legis Salicae, Text und Übersetzung, Göttingen 1959

ders.

Sachsenspiegel, Landrecht und Lehnrecht, Göttingen 1955

Endemann, Wilhelm

Das Civiprozeßverfahren nach der kanonistischen Lehre, ZfdZ 15 (1891), S. $177 \mathrm{ff}$.

Engelmann, Arthur/ Millar, Robert W. (Editor)

A History of Continental Civil Procedure, London 1927

Erler, Adalbert/ Kaufmann, Ekkehard (Hrsg.)

Handwörterbuch zur deutschen Rechtsgeschichte, Band I, Berlin 1971, Band II, Berlin 1978

Fehr, Hans

Deutsche Rechtsgeschichte, 6. Aufl., Berlin 1962

Ficker, Julius

Forschungen zur Reichs- und Rechtsgeschichte Italiens, Band III.1, 1870, Nachdruck Aalen 1961

Fitzherbert, Anthony/, Sir Matthew

The New Natura Brevium, 9. ed., London 1794

Flessner, Axel

Die Rechtsvergleichung als Kundin der Rechtsgeschichte, in:

ZEuP 7 (1999), S. 513 ff. 
Frank, Martin

Das verfahrenseinleitende Schriftstück in Art 27 Nr. 2 EuGVÜ, Lugano Übereinkommen und in Art. 6 Haager Unterhaltsübereinkommen 1973, Berlin 1998

Franklin, Otto

Das königliche Kammergericht vor dem Jahr 1495, Berlin 1871

Geimer, Reinhold/ Geimer, Ewald/ Geimer, Gregor

Internationales Zivilprozeßrecht, 5. Aufl., Köln 2004

ders.

Salut für die Verordnung (EG) Nr. 44/2001 (Brüssel I), in: IPrax 2002, S. $69 \mathrm{ff}$.

Glasser, Cyril

Civil Procedure and the Lawyers - The Adversary System and the Decline of the Orality Principle in: 56 (1993) M.L.R. S. 307 ff.

Günther, Gerhard

Die Anfänge der Rezeption des mittelalterlichen Privatrechts in Thüringen bis zur Mitte des 14. Jahrhunderts, Jena 1956

Hahn, Carl

Materialien zu den Reichsjustizgesetzen, Band II 1. Teilband, Berlin 1880

Hall, G. D. G. (Editor)

Glanvill, Ranulphus de: Tractatus de legibus et consuetudinibus regni Angliae qui Glanvilla vocatur, London, Edinburgh 1965

Harmer, Florence Elizabeth

Anglo-Saxon Writs, 2. ed., Stamford 1989

Hartmann, Peter

Zivilprozess 2001/2002: Hunderte wichtiger Änderungen, in: NJW 2001 S. $2577 \mathrm{ff}$.

, Oskar

Die Kunst des Sachvortrags im Zivilprozeß, Heidelberg 1988 
Hattenhauer, Hans

Europäische Rechtsgeschichte, 3. Aufl., Heidelberg 1999

Hellweg, August

Geschichtlicher Rückblick über die Entstehung der deutschen Zivilprozeßordnung, in: AcP 61 (1878), S. 78 ff.

$H e ß$, Burkhard

Aktuelle Perspektiven der europäischen Rechtsangleichung, in: JZ 2001, S. $573 \mathrm{ff}$.

ders.

Die „Europäisierung“ des internationalen Zivilprozessrechts durch den Amsterdamer Vertrag - Chancen und Gefahren, in: NJW 2000, S. 23 ff.

ders.

Die Zustellung von Schriftstücken im europäischen Justizraum, in: NJW 2001, S. 15 ff.

Holdsworth, Sir William S.

A History of English Law, London,

Vol. I: 7. ed. 1956; Vol. II: 4. ed. 1936; Vol. III: 4. ed. 1936; Vol. V: 3. ed. 1944, reprinted 1966; Vol. VII: 2. ed. 1936, reprinted 1966; Vol. IX: 3. ed. 1944, reprinted 1966; Vol. XV: 1965

ders.

The Development of written and oral Pleading, in: Selected Essays in Anglo-American Legal History II, S. 614 ff., Boston 1908, Neudruck Frankfurt am Main 1968

Hollond, H.A.

Writs and Bills, Cambridge Law Journal 8 (1942/44) S.

Holmes, O.W.

Early English Equity, in: Selected Essays in Anglo-American Legal History II, S. 705 ff., Boston 1908, Neudruck Frankfurt am Main 1968

Homeyer, Karl Gustav (Hrsg.)

Richtsteig Landrechts nebst Cautela und Premis, Berlin 1857 
Ipsen, Jörn

Staatsrecht I, 6. Aufl., Neuwied, Kriftel, Berlin 1994

James, Philip S.

Introduction to English Law, 12. ed., London 1989

Jacob, Sir I. H.

The English System of Civil Procedure in: Common Market Law Review 1 (1963/64), S. 294 ff.

ders.

The Reform of Civil Procedure Law, London 1982

Kaser, Max/ Hackl, Karl

Das römische Zivilprozeßrecht, 2. Aufl., München 1996

Kaufmann, Erich

„Ein Mann- ein Wort“, in: JuS 1961, S. 120 ff.

Kaufmann, Horst

Zur Geschichte des aktionenrechtlichen Denkens, in: JZ 1964, S. 482 ff.

Kengyel, Miklos

Zivilprozessrecht um die Jahrtausendwende, in: ZVglRWiss 101 (2002),

S. $260 \mathrm{ff}$.

Kerameus, Konstantinos D.

Angleichung des Zivilprozeßrechts in Europa, in: RabelsZ 66 (2002),

S. $1 \mathrm{ff}$.

Kip, Hans

Das sogenannte Mündlichkeitsprinzip, Köln, Berlin 1952

Knöringer, Dieter

Die Assessorklausur im Zivilprozess, 11. Aufl., München 2005

Köbler, Gerhard

Deutsche Rechtsgeschichte, 6. Aufl., München 2005 
Kötz, Hein

Justiz in Deutschland und England: Ein Stilvergleich, in: JA1991, S. $257 \mathrm{ff}$.

Kollmann, Andreas

Begriffs- und Problemgeschichte des Verhältnisses von formellem und materiellem Recht, Berlin 1996

Kommission für ein europäisches Zivilprozessgesetzbuch

Text der Vorschläge zum europäischen Zivilprozeßgesetzbuch, in: ZZP 109 (1996), S. 345 ff.

Koschaker, Paul

Europa und das römische Recht, 3. Aufl., München Berlin 1958

Kropholler, Jan

Europäisches Zivilprozeßrecht, 7. Aufl., Heidelberg 2002

Kunz-Schmidt, Susanne

Das Unterschrifterfordernis für bestimmende Schriftsätze im Zivilprozeß, in: NJW 1987, S. 1296 ff

Langan, Peter/ Henderson, L.D.J.

Civil Procedure, 3. ed., London 1983

Langdell, Christopher C.

The Development of Equity-Pleading from Canon Law Procedure, in: Selected Essays in Anglo- American Law History II, Boston 1907, Neudruck Frankfurt am Main 1968, S. 759

Leipold, Dieter

Vereinfachung und Beschleunigung des Rechtsschutzes durch summarische Verfahren - Funktionale und rechtsvergleichende Analyse von Summary Judgment, Interim Payment, Payment into Court und Striking Out im englischen Zivilprozeßrecht auf der Grundlage des Berichts von Adrian A.S. Zuckerman Verfahrensinstitute zur Prozeßbeschleunigung im englischen Zivilprozeßrecht, Köln 1998

Lemken, Elmar

Diskussionsbericht zum europäischen Zivilprozeßrecht, in: ZZP 109 (1996), S. 337 ff. 
Leonhardt, Gerhardt

Zur Reform des Zivilprozesses in Deutschland, Hannover 1865, Neudruck: Aalen 1982

Lindacher, Walter F.

Europäisches Zustellungsrecht, in: ZZP 114 (2001), S. 179 ff.

Löwenkamp, Hans

Die Lehre vom Schriftsatz in ausländischen Zivilprozeßsystemen, Diss. Erlangen-Nürnberg 1965

Maitland, Frederic W.

Cam, Helen (Editor)

Selected Historical Essays of F. W. Maitland, Cambridge 1957

ders.

Lectures on the Forms of Action at Common Law, Cambridge 1936, reprinted Cambridge 1984

ders.

Fischer, H.A.L. (Editor)

The Collected Papers of Frederic William Maitland, Vol. II, Cambridge 1911

Malterer, Michael

Lord Woolf's Access to Justice, Hamburg 2000 (Diss. Regensburg 1999)

Maunz, Theodor/ Dürig, Günter/ Herzog, Roman

Grundgesetz, Loseblattsammlung, München ,Stand 2001

May, J./ Scott, Richard (Editors)

Civil Procedure Vol. I; London 2000

Michalik, Paul

Justice in Crisis: England and Wales, in: Civil Justice in Crisis - Comparative Perspectives in Civil Procedure; Zuckerman, Adrian (Editor); Oxford 1999 S. 117 ff.

Michel, Helmut/ Seipen, Christoph von der

Der Schriftsatz des Anwalts im Zivilprozeß, 5. Aufl., München 2000 
Milsom, Stroud F. C.

Historical Foundations on the Common Law, 2. ed., London 1981

Mittermaier, Carl J.A.

Der neue Entwurf einer Civilprozeßordnung für das Königreich Bayern verglichen mit den Leistungen in anderen Staaten und im Zusammenhang mit der Frage über die Einführung einer gemeinschaftlichen Civilprozeßordnung für Deutschland, in: AcP 45 (1862), S. 99 ff.

Müchel, Hugo

Das Verfahren bis zur Litis-Contestation im ordentlichen canonischen Civilprozess, Lepzig 1870

Muther, Theodor

Zur Geschichte des römisch- canonischen Prozesses in Deutschland, Berlin 1881

ders.

Römisches und kanonisches Recht im Mittelalter, Berlin 1879

Nicholas, Barry

Besprechung von Peter, Actio und Writ, in: IVRA 9 (1958) S. 235 ff.

Nörr, Knut Wolfgang

Reihenfolgeprinzip, Terminsequenz und „Schriftlichkeit“; ZZP 85 (1972), S. $160 \mathrm{ff}$.

Ober, Leo

Die Rezeption der kanonischen Zivilprozeßformen und des Schriftlichkeitsprinzips im geistlichen Gericht zu Strassburg, in: Archiv für katholisches Kirchenrecht 90 (1910), S. 599 ff.

Oberheim, Rainer

Zivilprozeßrecht für Referendare, 6. Aufl. Düsseldorf 2004

Odgers, William Blake (Editor) et al.

A Century of Law Reform, London 1901

O’Hare, John/ Browne, Kevin/ Hill, Robert

Civil Litigation, 9. ed., London 2000 
Ott, Emil

Das Eindringen des canonischen Rechts, seine Lehre und wissenschaftliche Pflege in Böhmen und Mähren während des Mittelalters, in: ZRG Kan. 3 (1913), S. 1 ff.

Peter, Hans

Actio und Writ, Tübingen 1957

Pollock, Frederic/ Maitland, Frederic W.

The History of Common Law before the Time of Edward I, Vol. I \& II, 2. ed., Cambridge 1898, reissued 1989

Potter, Harold/ Kiralfy, A.K.R. (Editor)

Potter's Historical Introduction to English Law, 4. ed., London 1958

\section{Ranieri, Filippo}

Besprechung von Bieresborn, Klage und Klageerwiderung im deutschen und englischen Zivilprozess, in: Ius Commune, 28 (2001), S. 361 ff.

Reimann, Mathias

Rechtsvergleichung und Rechtsgeschichte im Dialog, in: ZEuP 7 (1999), S. $496 \mathrm{ff}$.

Reynold, Heinrich

Justiz in England, Köln 1968

Rosenberg, Leo/ Schwab, Karl Heinz/ Gottwald, Peter

Zivilprozeßrecht, 16. Aufl., München 2004

Roth, Herbert

Die Vorschläge der Kommission für ein europäisches Zivilprozeßgesetzbuch - das Erkenntnisverfahren, in: ZZP 109 (1996), S. 271 ff.

Rumberg, Carsten/ Eicke, Tim

Der „Woolf Report“ - Reform des englischen Zivilprozessrechts in: RIW 1998, S. 19 ff. 
Samuel, Geoffrey

System und Systemdenken- $\mathrm{Zu}$ den Unterschieden zwischen kontinentaleuropäischem Recht und Common Law, ZEuP 3 (1995) S. 375 ff.

Schellhammer, Kurt

Zivilprozess, 8. Aufl., Heidelberg 1999

Schelo, Sven

Rechtsangleichung im Europäischen Zivilprozeßrecht: EG-Kompetenzen und Modellgesetz, Diss. Münster 1999

Schilken, Eberhard

Die Vorschläge der Kommission für ein europäisches Zivilprozeßgesetzbuch - einstweiliger und summarischer Rechtsschutz und Vollstreckung, in: ZZP 109 (1996), S. 315 ff.

Schilling, Bruno

Corpus Iuris Canonici Bd. I-II, in seinen wichtigsten und anwendbarsten Theilen in's Deutsche übersetzt und systematisch zusammengestellt von

Dr. Bruno Schiling, Band 1 und 2, Leipzig 1884

Schlosser, Hans

Spätmittelalterlicher Zivilprozeß nach bayerischen Quellen, Köln, Wien 1971

Schmidt, Kathrin

Der Abschied von der Mündlichkeit, der Parteiherrschaft und dem Überraschungsprinzip - Die Reform des Zivilprozesses am High Court of Justice in den neunziger Jahren; Frankfurt am Main 1997

Schmidt, Richard

Die Klagänderung, Leipzig 1888

ders.

Lehrbuch des deutschen Zivilprozeßrechts, 2. Aufl., Leipzig 1906

Schmitthoff, Clive M.

Der Zivilprozeß als Schlüssel zum englischen Rechtsdenken, in: JZ 1972 , S. $38 \mathrm{ff}$. 
Schröder, Richard/ Künßberg, Eberhard von

Lehrbuch der deutschen Rechtsgeschichte, 7. Aufl., Berlin 1932

Schulz, Fritz

The writ ,praecipe quod reddat“ and its continental models, in: 54 (1942) Juridical Review, S. 1 ff

Schwerin, Claudius von/ Thieme, Hans

Grundzüge der deutschen Rechtsgeschichte, 4. Aufl., Berlin 1950

Schwartz, Johann C.

400 Jahre deutscher Civilprozeßgesetzgebung, Berlin 1898

Siegel, Heinrich

Die Gefahr vor Gericht und im Rechtsgang, Wien 1866

ders.

Geschichte des deutschen Gerichtsverfahrens, Gießen 1857, Nachdruck 1970

Sim, Raymond S./ Pace, P.J.

“A“ level English Law, 7. ed. London 1991

Sobich, Philip

Die Civil Procedure Rules 1999 - Zivilprozeßrecht in England, in: JZ 1999, S. 775 ff.

Stafyla, Athena

Die Rechtshängigkeit des EuGVÜ nach der Rechtsprechung des EuGH und der englischen, französischen und deutschen Gerichte, Sinzheim 1998

Stein/Jonas

Bork, Reinhard (Hrsg.)

Zivilprozeßordnung, Band III, 21. Aufl. Tübingen 1997

Stein, Peter

Besprechung von Hans Peter - Actio und Writ, in: Studia et Documenta 24 (1958), S. 335 ff. 
Steins, Achim

Der ordentliche Zivilprozeß nach den Offizialstatuten, in: ZRG Kan. 59 (1973) S. $191 \mathrm{ff}$.

Stobbe, Otto

Geschichte der deutschen Rechtsquellen, Band II, Braunschweig 1864, Neudruck 1965

Stölzel, Adolf

Die Entwicklung des gelehrten Richterthums in deutschen Territorien, Band I, Stuttgart 1872, Neudruck Aalen 1964

Storme, Marcel (Hrsg.)

Rapprochement du Droit Judiciaire de l'Union européene - Approximation of Judiciary Law in the European Union, Dordrecht, Boston, London 1994

ders.

Rechtsvereinheitlichung in Europa - Ein Plädoyer für ein einheitliches europäisches Prozeßrecht, in: RabelsZ 56 (1992), S. 290 ff.

Stubbs, William (Editor)

Chronicles and Memorials of Great Britain and Ireland during the Middle Ages, London 1986

Stürner, Rolf

Die Aufklärungspflicht der Parteien des Zivilprozesses, Tübingen 1976

ders.

Der europäische Zivilprozeß - Einheit oder Vielfalt? in: Wege zu einem europäischen Zivilprozeßrecht, herausgegeben von Mitgliedern der juristischen Fakultät Tübingen, Tübingen 1992

ders.

Modellregeln für den internationalen Zivilprozeß, in: ZZP 112 (1999), S. $185 \mathrm{ff}$.

Stutz, Ulrich

Das Stadtrecht gegen die Formstrenge im Strafverfahren, in: ZRG Germ. 38 (1917), S. 367 ff. 
Sunderland, Edson R.

The English Struggle forProcedural Reform, in: Harvard Law Review 39, S. $725 \mathrm{ff}$.

Thomas, Heinz/ Putzo, Hans/ Reichold, Klaus/ Hüßtege, Reiner Zivilprozeßordnung, 27. Aufl., München 2005

Trusen, Winfried

Anfänge des gelehrten Rechts in Deutschland, Wiesbaden 1962

Vollkommer, Max

Richter und Gerichte in England, in: ZZP 73 (1960), S. 145 ff.

Vossius, Oliver

$\mathrm{Zu}$ den dogmengeschichtlichen Grundlagen der Rechtsschutzlehre, Ebelsbach 1985

Walter, Gerhard

Modellregeln für den internationalen Zivilprozeß - deutscher Text, in:

ZZP 112 (1999), S 204 ff.

Wassermann, Rudolf

Der soziale Zivilprozess, Neuwied, Darmstadt 1978

Weber, Helmut

Aktuelle Prozeßrechtsreformen in England, in: ZZPInt 2000, S. 59 ff.

Wesel, Uwe

Geschichte des Rechts, 2. Aufl., München 2000

Wetzell, Georg W.

System des ordentlichen Zivilprozesses, Leipzig 1878

Wieacker, Franz

Privatrechtsgeschichte der Neuzeit, 2. Aufl., Göttingen 1967

Zallinger, Otto von

Wesen und Ursprung des Formalismus im altdeutschen Prozeßrecht, Wien 1898 
Zeumer, Karl (Hrsg.)

Formulae Merowingici et Karolini Aevi, Monumenta Germaniae Historica, 1882- 1886, Nachdruck 1963 (MGH Form.)

ders. et al. (Hrsg.)

Leges Saxonum, Leges Thuringorum, Edictum Theoderici regis, Remedil curiensis episcopi capitula, Lex Ribvaria, Lex Frankorum Chamavorum, Lex Romana Raetica Curiensis, Monumenta Germaniae Historica, Leges Band 5 1875- 1889, Nachdruck 1965 (MGH Leges I, 5)

Zimmermann, Reinhard

Der europäische Charakter des englischen Rechts, in: ZEuP 1 (1993)

S. $4 \mathrm{ff}$

ders.

Einführung: Rechtsgeschichte, Rechtsvergleichung, Privatrechtsdogmatik, in: ZEuP 7 (1999), S. 494 f.

Zöller, Richard

Zivilprozeßordnung, 25. Aufl., Köln 2005

Zweigert, Konrad/ Kötz, Hein

Einführung in die Rechtsvergleichung auf dem Gebiet des Privatrechts, 3. Aufl., Tübingen 1997 


\section{Fallverzeichnis}

The Abidin Daver [1984] A.C. 398 (H.L.)

Afro Continental Nigeria v. Meridian Shipping Co S.A. (The Vrontados) [1982] Lloyd's Rep. 241

Banque et Francaise v. Clarke [1894] W.N. 203

Barclays Bank of Swaziland Ltd. v. Hahn [1989] 1 W.L.R. 506

Baroda v. Wildenstein [1972] 2 Q.B. 283

Battersby v. Anglo-American Oil Co Ltd. [1945] K.B. 23

Brady v. Barrow Steelworks Ltd. [1965] 2 Q.B. 182

British Airways Board v. Laker Airways [1985] A.C. 58 (H.L.)

Brophy v. Dunphys Chartered Surveyors, The Times, 11. 03.1998 (C.A.)

Bruce v. Odhams Press Ltd. [1936] All ER 294

Bryant v. Herbert [1878] 3 C.P.D. 390

Burmah Oil Co. v. Governor and Co. of the Bank of England [1971] 1 W.L.R. 473

Castanho v. Brown and Root (UK) Ltd. [1981] A.C. 557 (H.L.)

Colt v. Sarlie [1966] 1 W.L.R. 440

Davie v. New Merton Board Mills Ltd. [1965] 1 All ER 379

Dresser (UK) Ltd v. Falcongate [1992] All ER 450 (C.A.)

Drane v. Evangelou [1978] 1 W.L.R. 455

Earl of Oxford [1615] 1 Ch. Rep. 6

Fowler v. Lanning [1959] 1 Q.B. 426

Hastie \& Jenkerson v. McMahon [1991] All ER 255

Heaven v. Road \& Rail Wagons Ltd. [1965] 2 Q.B. 355

Independent Automatic Sales Ltd. v. Knowels \& Foster [1962] 1 W.L.R. 974

Molins plc v. GD SpA [2000] 1 W.L.R. 1741

Neste Chemicals v. DK Line [1994] Lloyd's Rep. 6; [1994] 3 All E R 180 (C.A.)

Remmington v. Scoles [1897] 2 Ch. 1

Restick v. Crickmore [1994] 1 WLR 420 (C.A.)

Smith v. Probyn (2000) The Times, 29. 03. 2000

Spiliada Maritime Corporation v. Cansulex Ltd. [1987] A.C. 460 (H.L.)

Thompson v. Brown [1981] 1 WLR 744

Thomson v. Pheney (1832) 1 Dowl. Pr. Cas. 441 
Trow v. Ind Coope (West Midlands) [1967] 2 Q.B.567

Vinos v. Marks and Spencer plc (2000) The Independent, 17. 07. 2000 


\section{Lebenslauf}

Thomas Schuster,

geboren am 04.10.1974 in Bukarest/Rumänien

verheiratet

Abitur 1994 in Clausthal-Zellerfeld

Studium von 1994-2001 in Göttingen, Thessaloniki/Griechenland und

London/Großbritannien

1. Staatsexamen am 22.09.1999

LLM (International Business Law) an der University of London im September 2001

Referendariat von 2002-2004 in Darmstadt, Würzburg und Frankfurt am Main

2. Staatsexamen am 22.04.2004

Juni-August 2004 angestellter Rechtsanwalt in Frankfurt am Main

Seit Oktober 2004 Staatsanwalt bei der Staatsanwaltscheft Ansbach 

Berichterstatter: Professor Dr. Joachim Münch

Mitberichterstatter: Professor Dr. Gerald Spindler

Tag der mündlichen Prüfung: 13. Dezember 2005 